University of Louisville

ThinkIR: The University of Louisville's Institutional Repository

Electronic Theses and Dissertations

$12-2018$

\title{
Transcending the panels: varieties of experience and selfhood in comics.
}

Will D. Simpson

University of Louisville

Follow this and additional works at: https://ir.library.louisville.edu/etd

Part of the Esthetics Commons

\section{Recommended Citation}

Simpson, Will D., "Transcending the panels: varieties of experience and selfhood in comics." (2018). Electronic Theses and Dissertations. Paper 3117.

https://doi.org/10.18297/etd/3117

This Doctoral Dissertation is brought to you for free and open access by ThinkIR: The University of Louisville's Institutional Repository. It has been accepted for inclusion in Electronic Theses and Dissertations by an authorized administrator of ThinkIR: The University of Louisville's Institutional Repository. This title appears here courtesy of the author, who has retained all other copyrights. For more information, please contact thinkir@louisville.edu. 
TRANSCENDING THE PANELS:

\title{
VARIETIES OF EXPERIENCE AND SELFHOOD IN COMICS
}

By

Will D. Simpson

B.A., Western Kentucky University, 2010

M.A., Western Kentucky University, 2013

\begin{abstract}
A Dissertation
Submitted to the Faculty of the

College of Arts and Sciences of the University of Louisville in Partial Fulfillment of the Requirements

for the Degree of

Doctor of Philosophy

in Humanities

Department of Comparative Humanities

University of Louisville

Louisville, Kentucky
\end{abstract}

December 2018 

TRANSCENDING THE PANELS:

VARIETIES OF EXPERIENCE AND SELFHOOD IN COMICS

By

Will D. Simpson

B.A., Western Kentucky University, 2010

M.A., Western Kentucky University, 2013

A Dissertation Approved on

November 16, 2018

by the following Dissertation Committee:

John Gibson, PhD

Andreas Elpidorou, $\mathrm{PhD}$

Ranen Omer-Sherman, $\mathrm{PhD}$

Michael Hagan, PhD

Christopher Bartel, $\mathrm{PhD}$ 


\section{ACKNOWLEDGEMENTS}

I would like to thank those faculty members with whom I have worked closely, and who have helped me immensely in furthering my academic interests over the past four years. Thanks to Dr. John Gibson and Dr. Andreas Elpidorou for supporting my philosophical explorations and for being highly engaged in the dissertation process and the course work that preceded it. Thanks to Dr. Ranen Omer-Sherman for providing support and immensely helpful comments and critiques on the dissertation as it progressed. Thanks to Dr. Christopher Bartel for offering perspective on the finished project as well as your time. Thanks to Dr. Michael Hagan for sitting as a committee member and also for many great conversations over the course of my time at the university. Also, thank you to my fellow teaching graduate employees for the great friendships and memories. And finally, thanks to my partner Maggi, my parents, and my friends who are always supportive and wonderful. 


\section{ABSTRACT \\ TRANSCENDING THE PANELS: VARIETIES OF EXPERIENCE AND SELFHOOD IN COMICS \\ Will D. Simpson}

November 16, 2018

In this dissertation, I argue that the typical formal features of comics in the American tradition over the past century have influenced the types of narrative content that tend to be communicated by said medium. I argue that the types of reader experiences that are afforded by the comics form, in part, shape the types of stories told through comics. The experiences that result from the ways we engage perceptually, cognitively, emotionally, and conceptually with comics imply a certain view of selfhood that is potentially subversive in the context of American cultural religiosity and spirituality.

The formal features of comics, and the resulting reader experiences, imply an understanding of selfhood as being conventional, narrativized, and made possible by active interpretation. The view that selves are constituted by narratives also can be found in the work of various philosophers of self. Narrative understandings of selfhood stand in stark contrast to the traditional entrenched Western view that selves consist of the unified and continuous essences of individuals. Because comics' formal features highlight the actively interpreted and constructed nature of the selves of characters in comics, they are fitting for the communication of narratives that engage with traditions of thought in 
which selves are considered to be malleable, interpretable, and narrative in nature. This includes many traditions of occultism and esotericism.

Chapter one examines readers' typical perceptual and cognitive engagements with the comics form and expounds the process of "closure" as a means by which readers understand a comic as representing a coherent storyworld. Chapter two offers a theoretical model of emotions as processes, which can best account for the range of emotional affordances offered by comics' character depictions, artistic and design elements, and the processes that constitute closure. Chapter three illuminates the conceptual implications of the perceptual, cognitive, and emotional affordances of comics, arguing that comics imply a conventional and narrativized understanding of selfhood. Finally, chapter four examines the American cultural history of comics and highlights examples of esoteric and occultist themes and traditions appearing in ways that highlight a narrative understanding of selfhood. 


\section{TABLE OF CONTENTS}

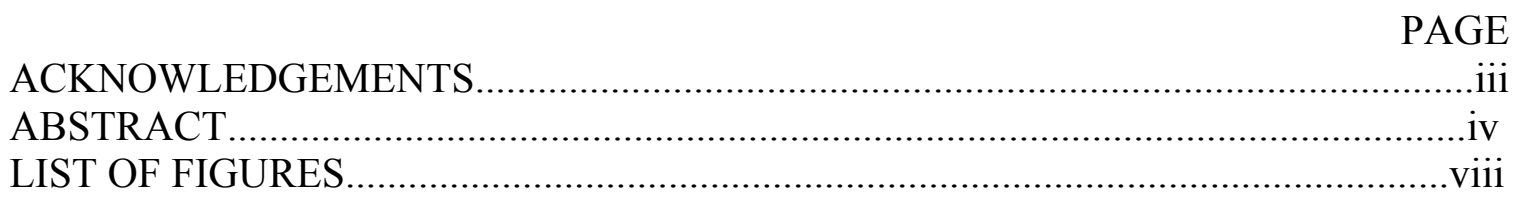

INTRODUCTION: FORM AND CONTENT ......................................................

Typical Formal Features of Comics..............................................................4

Comics versus Visual Language................................................................

I: CLOSURE AS NON-SENSORIAL COGNITION OF DEPICTED COHERENCE....12

Imagination, Inference, and Comics...........................................................12

Closure: Notable Approaches....................................................................16

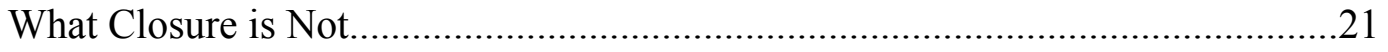

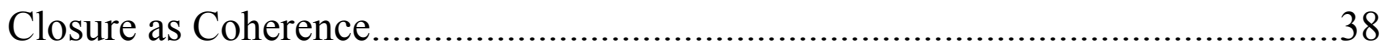

Local and Global Coherence via Closure: Imagination and Inference.................43

Distinction Between Comics and Film.........................................................48

Two Examples of Depicted Coherence.........................................................51

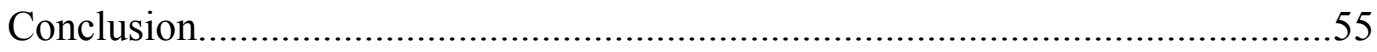

II: AESTHETIC POSSIBILITIES OF EMOTIONS IN COMICS ...............................57

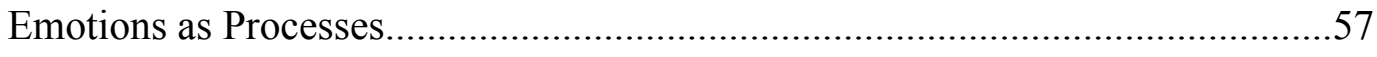

Narrative, Time, and Emotional Responses..................................................70

Emotional Salience in Comics Through Character Depiction.............................75 
Emotional Salience Through Artistic Style and Design Elements.......................89

Closure, Imagination, and Emotion..............................................................96

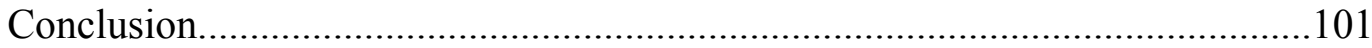

III: COMICS AND THE DISSOLUTION OF THE TRADITIONAL SELF.................103

Traditional Understandings of Self............................................................103

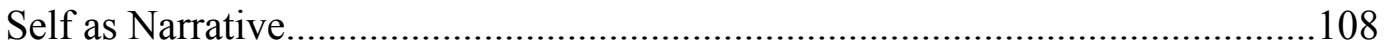

Character Depiction and Selfhood............................................................. 119

Visual Artistic and Design Elements and Selfhood..........................................130

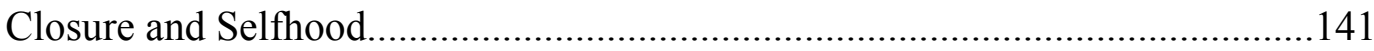

Potential Upsets in Readers’ Experiences of Self............................................144



IV: OCCULTISM, ESOTERICISM, AND THE COMICS FORM..............................154

The Illusory Nature of Selfhood and Traditions of Occultism and Esoterica......154

Superheroics and Narrative Identity .......................................................162

Science Fiction, the Fantastical, and the Humbled Self.....................................172

Horror Comics, Youth Culture, and Subversive Values...................................183

Occultist Views of Selfhood, Reader Consciousness, and Comics....................196

Conclusion: Form and Content...................................................................206

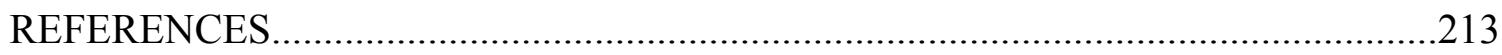

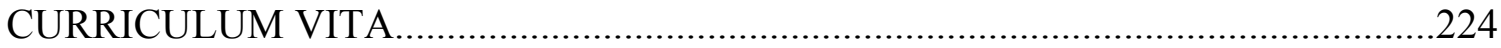




\section{LIST OF FIGURES}

$\begin{array}{lll}\text { FIGURE PAGE } & \text { PAR }\end{array}$

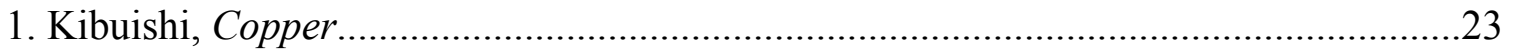

2. McCloud, Understanding Comics, 65..............................................................26

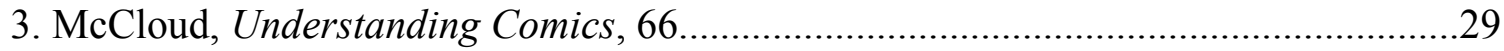

4. Moore and Bolland, Batman: The Killing Joke......................................................33

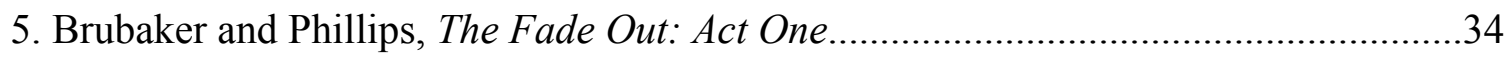

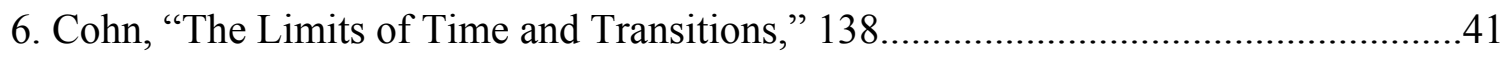

7. Siegel and Schuster, The Superman Chronicles Volume One ...................................52

8. Loeb and Sale, Superman for All Seasons, DC Comics...........................................54

9. Brubaker and Mahnke, Batman: The Man Who Laughs, DC Comics..........................60

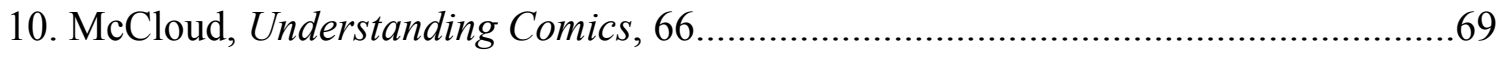

11. Moore and Gibbons, Watchmen, Chapter VI, 26............................................... 78

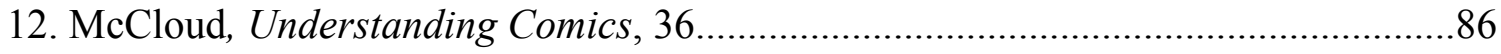

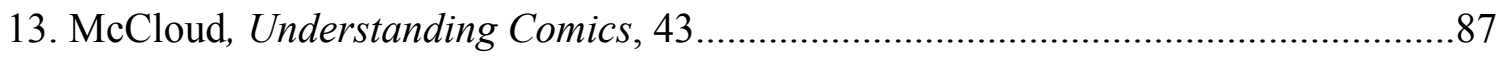

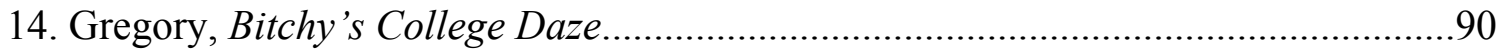

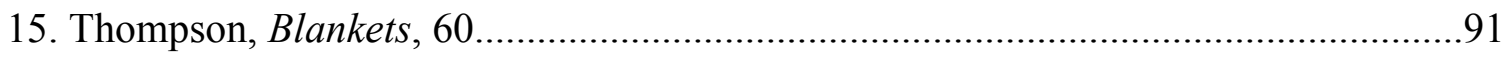

16. Ware, Jimmy Corrigan: The Smartest Kid on Earth.............................................94

17. Relations of comic books to emotional and cognitive elements of reader

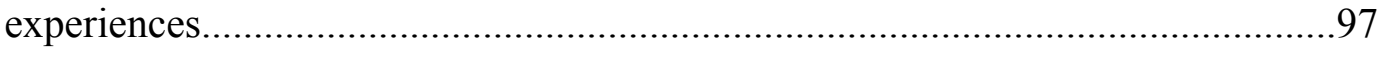

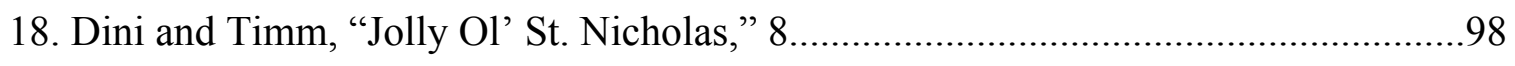


19. O’Neil and Adams, “The Joker's Five-Way Revenge," 173 …………………….......122

20. Moore, et al., "The Anatomy Lesson," 21............................................................131

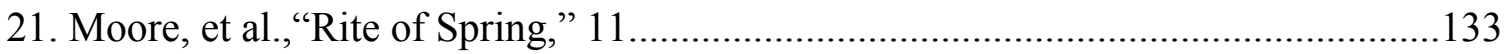

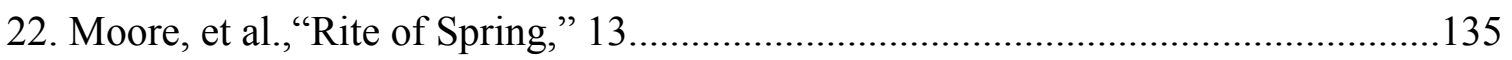

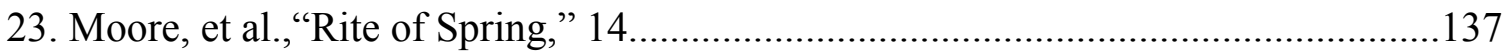

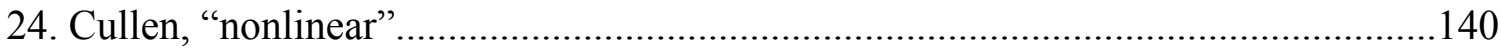

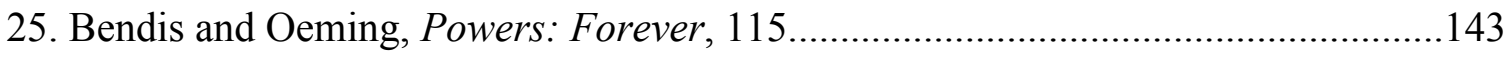

26. Morrison, The Invisibles: The Invisible Kingdom, 275 ...........................................150

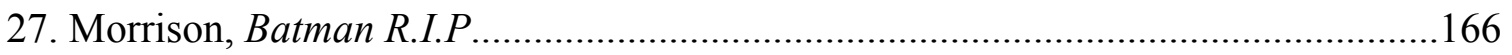

28. Leger and Reuths, "Doctor Occult: The Ghost Detective," ........................................170

29. Leger and Reuths, "Doctor Occult: The Ghost Detective,".........................................171

30. “The Meteor Monster,” Weird Science \#13 ............................................................175

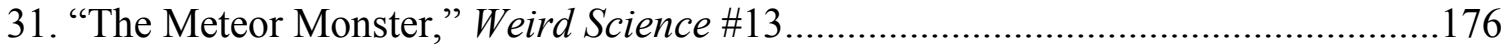

32. Moreci, Roche Limit Volume 1: Anomalous...........................................................178

33. Moreci, Roche Limit Volume 1: Anomalous..........................................................180

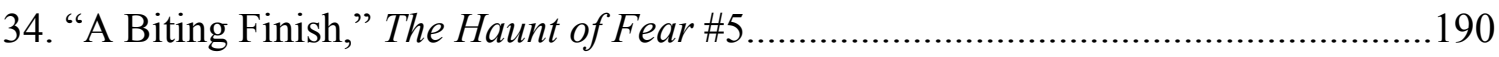

35. “The Living Death!” Tales From the Crypt \#24.......................................................191

36. Wolverton, "Nightmare World," Weird Tales of the Future \#3.................................193

37. Wolverton, "Nightmare World," Weird Tales of the Future \#3................................194

38. Wolverton, "Nightmare World," Weird Tales of the Future \#3................................195

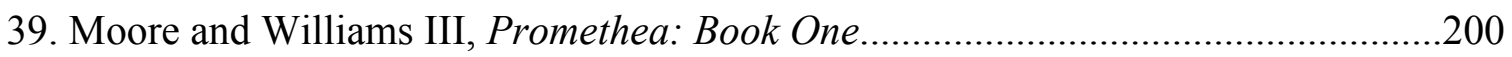

40. Moore and Williams III, Promethea: Book Five .....................................................203

41. Moore and Williams III, Promethea: Book Five ...................................................203 
42. Moore and Williams III, Promethea: Book Five ...................................................205 


\section{INTRODUCTION: COMICS FORM AND CONTENT}

The medium of comics-that is, narratives conveyed through a combination of sequences of static images and text-in the United States has often been conflated with the genres of stories and particular narrative tropes that have brought the most attention to the medium. ${ }^{1}$ Beginning in the 1930 's with the explosive rise in popularity of the superhero genre, the comics medium has had a difficult time outrunning the influence of the cultural attitudes toward the escapist and, as some would argue, infantile superhero and fantasy stories. While autobiographical memoirs, war narratives, and slice-of-life dramas have been staples of art and independent comics movements, comics as a medium often is popularly conceived of as "juvenile, disposable trash."2

But why has the comics medium historically been conflated with the influential genres conveyed through that medium? Why do we associate comics with fantastical heroes, alien worlds, magical realms, and horrific monsters? It would be strange to refer to a film based on a novel as a "novel film," whereas movies featuring superheroes and

\footnotetext{
${ }^{1}$ Aaron Meskin, Roy T. Cook, and Frank Bramlett, introduction to The Routledge Compantion to Comics, ed. Frank Bramlett, Roy T. Cook, and Aaron Meskin (New York: Routledge, 2017), 3. "It is worth noting that, for more than 100 years, comics were created via the application of pencil, pen, or brush to paper, and comics itself seems best characterized as a medium, despite its continued identification or strong association with one or a very few influential genres (e.g. superhero comics or funny animal comics)."

${ }^{2}$ Rocco Versaci, This Book Contains Graphic Language: Comics as Literature (London: Continuum Intl. Publishing Group, 2007), 2.
} 
other fantastical elements in any capacity are often referred to as comic book movies. In the following work, I will explore the relationship between the form and content of comics. I will claim that there is an underlying connection between the fantastical and mythical themes that are commonly associated with comics and the ways in which readers engage with comics.

While comics studies is becoming increasingly enfranchised in academic environments, academic explorations of comics have tended to bifurcate toward one of two routes: either historical and cultural examinations of the narrative content of certain comics, or formalist approaches to comics as an art form, independent of narrative content. Here, I will draw connections between these two approaches, arguing that the typical formal features of comics in the U.S. context have cognitive, emotional, and conceptual implications that make comics' associations with fantastical and mythical narrative tropes intelligible, and even likely. In the following chapters, I will utilize a variety of approaches to comics (e.g., formalist and historical) and my own analysis will be primarily philosophical and cultural. In this way, I will tie together the particular philosophical implications of many formalist insights into the comics medium and the cultural understanding of the types of stories that comics often tell.

I will gradually broaden the scope of my analysis over four chapters, beginning with the fine-grained cognitive and perceptual experiences of readers and widening to eventually examine the cultural history of comics in the U.S. In the first chapter, I will explore many of the ways that readers tend to engage with comics. The ways in which people read comics is, to a large extent, conditioned on the artistic and narrative form of comics. Readers' perceptual experiences with the medium entail certain types of 
cognitive processing of the information contained in the comic. Readers' typical perceptual experiences and cognitive processing of the content of comics makes possible particular types of emotional engagement, as well.

In chapter two, I will slightly broaden the scope of my analysis to examine the types of emotional responses that comics typically make possible. I will argue that, while many understandings of the nature of emotional responses are available, a particular model of emotions as processes that involve both physiological and conceptual elements yields the most insight into the relation of emotions and comics. The formal features that I will examine, with regard to emotions, are character depiction, artistic and design elements, and the need for readers to enact closure. Through this, I will show how comics offer unique opportunities for readers to emotionally engage.

The third chapter will focus on the implications of comics for the concept of the self. After having examined readers' perceptual, cognitive, and emotional engagements with comics, I will show that the ways in which we read comics can influence the understanding of selves that comics imply. In this chapter, I will broaden my scope from the philosophical and formalist to the cultural and historical, as the concept of a "self" has particular implications within a given cultural context. Rather than affirming the common Western notion that selves are the enduring and unified essences of people, comics imply that selves are actively narrativized and potentially disunified. The implications of comics, then, converge with the understanding of selfhood offered by certain philosophers who maintain that selves are ultimately narrative in form.

In the final chapter, I will showcase several genres in the cultural history of American comics that narratively and artistically exemplify the implications for selfhood 
discussed in chapter three. Comics have been a historically likely source for fantastical, mythological, and potentially subversive narrative content. The picture of selfhood implied by the typical formal features of comics can help to make sense of the tendency for comics to contain occultist, esoteric, and religiously resonant narrative elements. With the conclusion of this work, I will draw together my analysis of the form of comics with much of the typical content of American comics. The types of stories and themes that many comics contain, I argue, makes sense in light of the ways that we engage with comics when we read them. Before we can begin this exploration, however, I will consider the definitional question of "comics."

\section{Typical Formal Features of Comics}

Comics rely on certain conventions in terms of their formal features (panels, gutters, speech balloons, etc.) within the various cultural contexts in which they appear. The issues addressed throughout this work will necessitate an exploration of the conventional formal features of those comics that have been most prominent in the cultural context of the United States throughout the Twentieth and Twenty-first centuries, as well as an exploration of the influence of those formal features on the potential cognitive, perceptual, emotional, and conceptual affordances provided to readers of comics.

While the formal features of comics may be similar across cultures (for example, the use of panels in both U.S. comics and Japanese manga), the conventional formal features differ enough that we should be wary of conflating distinct cultural traditions of comics by presuming that all comics afford readers the same types or ranges of 
experiences. Additionally, the uses and subsequent cultural implications of those formal features will differ according to their contexts.

Scott McCloud offered the most well known formalist definition of comics in his seminal work Understanding Comics: The Invisible Art. In it, McCloud writes that comics are best understood as "juxtaposed pictorial and other images in deliberate sequence, intended to convey information and/or to produce an aesthetic response in the viewer." ${ }^{3}$ This broad formal definition covers everything from graphic novels, to comic strips, to pre-Columbian Mexican picture manuscripts. ${ }^{4}$ While there are a number of potential problems with McCloud's definition, ${ }^{5}$ we need not wholeheartedly accept it for it to be helpful in examining the relevant formal features that typically appear in comics. For example, below I will side with Neil Cohn in claiming that "comics" should be understood as a social, rather than a formal, category. ${ }^{6}$ However, regardless of the nature of the category of "comics," comics do have paradigmatic formal features that McCloud's definition helps to illuminate. For example, comics typically involve multiple juxtaposed images and text.

\footnotetext{
${ }^{3}$ Scott McCloud, Understanding Comics: The Invisible Art (New York: HarperCollins, 1993): 9.

${ }^{4}$ McCloud, 10.

${ }^{5}$ Dylan Horrocks, "Inventing Comics: Scott McCloud's Definition of Comics," Hicksville, June 2001, http://hicksville.co.nz/Inventing\%20Comics.htm. McCloud's definition reveals his evaluative prescriptions for the term "comics," rather than offering an attempt at an objective understanding of the use of the term over time. McCloud downplays the role of the modern history of the comics medium, attempting to legitimize the form by making the typical content irrelevant.

${ }^{6}$ Classifying a work as a "comic" is a way to cite certain social expectations about that work's formal features, readership, artistic value, cultural import, and potentially, content. Calling a work a "comic" does not reference necessary or sufficient aspects of the structuring or linguistic elements of that work.
} 
The images that comprise a comic are typically presented in panels. The term "panel" refers to the sometimes-explicitly-bordered, typically rectangular, segmented portions of the comics page that visually distinguish or differentiate "certain moments of prime action from the imagined story [in] a discrete space.," Panels need not have explicit lined borders, though they often do. They may be distinguished by the appearance of blank spaces (either white spaces or ones filled in with color) between the images. Panels may also overlap, be only loosely implied visually, be non-rectangular, appear in various sizes, and take up multiple pages. ${ }^{8}$

Panels usually structure the narrative content of the comic and influence certain elements of reader experiences such as perspective, ${ }^{9}$ narrative relevance, emphasis, and reading time. As the seminal comics creator and theorist Will Eisner claims, the "number and size of the panels $[\ldots]$ contribute to the story rhythm and passage of time. ${ }^{\prime 10}$ Eisner, here, refers to both the passage of actual time for the reader and the depiction of the passage of narrative time within the storyworld of the comic.

The use of panels typically implies the presence of gutters, or separating spaces between panels. Like panels, gutters do not appear in a uniform fashion in all comics, but rather are rendered in numerous ways depending on the choices of the comic's creator or creative team. The typical appearance of the gutter is as a small blank space separating

\footnotetext{
${ }^{7}$ Randy Duncan and Matthew J. Smith, The Power of Comics: History, Form \& Culture (New York: Bloomsbury Academic, 2015): 131.

${ }^{8}$ Thierry Groensteen, The System of Comics (Jackson, MS: The University Press of Mississippi, 2007): 28-29.

${ }^{9}$ Will Eisner, Comics and Sequential Art (New York: W.W. Norton \& Company, 1985), 92. Will Eisner cites perspective as a means for influencing readers' "emotional states" and claims that we, as readers, are responsive to such artistic decisions because "we are responsive to environment."

${ }^{10}$ Eisner, 30.
} 
juxtaposing panels. Douglas Wolk details a few of the various types of gutters, writing, "some comics $[\ldots]$ have only a line between panels, or dissolve into blankness without specific panel borders. The bordered gutter has become the default though, because it's the clearest indication that there's a distinction between where the panel's image goes and where it doesn't." ${ }^{\prime 11}$ Panels and gutters are the formal features that most clearly enable readers to enact "closure," the cognitive process that facilitates much of the reading experience of those who engage with comics, and also the focus of chapter one.

Other common formal features of comics include thought bubbles and speech balloons. ${ }^{12}$ These are typically rounded spaces usually appearing within panels and containing narratively relevant text. The text is usually best understood as the speech or thought of a character that appears in the storyworld of the comic and the relevant character is often indicated by a "tail" of the balloon or thought cloud. ${ }^{13}$ Narration is often included by means of square captions that lack tails and, therefore designate no speaker that is internal to the scene depicted within the content of the panel in which the caption is included. Tailless captions can also contain text that represents the perspective of an unnamed narrator. In chapter one, I will discuss the relevance of these formal features (especially panels and gutters) in relation to the cognitive processes that underlie the enactment of closure.

\footnotetext{
${ }^{11}$ Douglas Wolk, Reading Comics: How Graphic Novels Work and What They Mean (Philadelphia, PA: Da Capo Press, 2007): 131.

${ }^{12}$ David Carrier, The Aesthetics of Comics (University Park, PA: Penn State University Press, 2000), 7. Carrier even goes as far as to define a "comic as a closely grouped sequence of images using balloons."

${ }^{13}$ Neil Cohn, The Visual Language of Comics: Introduction to the Structure and Cognition of Sequential Images (New York: Bloomsbury Publishing, 2013), 35.
} 
Comics versus Visual Language

Before moving forward, it will be useful to explore a key distinction articulated in detail by cognitive linguist Neil Cohn between the medium of comics and the languages in which comics are written. This issue parallels theoretical discussions regarding the definitional boundaries of the term "comics." While I will not presume to make any claims about necessary and sufficient conditions for the instantiation of comics in any particular case, the nature of the category of "comics" will influence the forthcoming investigation of the nature and potential range of reader experiences when engaging with that category.

Cohn takes comics, intuitively, to be "images and text, most often with the images in sequence. However, comics utilize these forms in a variety of different ways." ${ }^{14}$ For example, some comics communicate their content primarily through their visual artistic elements, with the text playing a supporting or supplemental role. Others are "dominated by text, relegating the images to illustrative roles." ${ }^{15}$ And still others utilize both images and text roughly equally in the communication of meaning. This intuitive notion is uncontroversial, but Cohn's claims about the relationship of comics to language push against many of the earlier ideas about that relationship.

Cohn claims emphatically "Comics are not a language." ${ }^{16}$ Other comics theorists have suggested that comics do constitute a "language," due to their structural nature and the experience of many readers that they become more fluent and confident in their reading of comics over time. In part, this recurring claim is utilized as a tactic for

\footnotetext{
${ }^{14}$ Neil Cohn, "Un-Defining 'Comics': Separating the Cultural from the Structural in 'Comics,"' International Journal of Comic Art 7, no. 2 (2005): 1.

${ }^{15}$ Cohn, "Un-Defining 'Comics,"” 1.

${ }^{16}$ Cohn, The Visual Language of Comics, 2.
} 
legitimizing an artistic medium that has repeatedly been designated as unimportant or aesthetically irrelevant. It is not clear that the theorists who use this comparison believe comics literally constitute a language or whether they are simply noting the similarities of structure. ${ }^{17}$ However, Cohn's work serves to counter any potential literal readings of these theorists by clarifying the distinction between elements of natural languages and comics as cultural artifacts. Cohn writes, "these analyses impose 'language' as a metaphor onto their systems of choice as a method of interpretation." ${ }^{, 18}$ Obviously, languages are used in the construction of comics (e.g., the textual elements of comics clearly exemplify the use of language). Cohn's point is that "comics" is a category of cultural artifacts, but also that the visual artistic elements of comics alone constitute a language literally. So, in his view, comics are not a language (just as novels are not a language), but they typically are written in two languages-namely, the textual language included in the comic, as well as the "visual language"19 exemplified by the structure and content of the visual art within the work. ${ }^{20}$

So, those theorists who have used the metaphor of "language" to describe comics are, according to Cohn, making a category error. Visual language, being a natural language, is amenable to linguistic and psychological examination that is broadly a-

\footnotetext{
${ }^{17}$ Eisner, Comics and Sequential Art, 1. Eisner uses scare quotes, writing, "comics communicate in a 'language' that relies on a visual experience." Darren Hudson Hick, "The Language of Comics," in The Art of Comics: A Philosophical Approach, eds. Aaron Meskin and Roy T. Cook (Malden, MA: Blackwell Publishing, 2012): 125-144.

And Hick only claims that, while comics cannot be understood as natural languages, "it is not unreasonable to discuss [comics] as being in many ways language-like, or as constituting a pseudo-language."

${ }^{18}$ Cohn, "Un-Defining 'Comics,", 2.

${ }^{19}$ Cohn, The Visual Language of Comics, 3 .

${ }^{20}$ Cohn, The Visual Language of Comics, 1. "Comics are social objects created by incorporating the results of two human behaviors: writing and drawing."
} 
cultural and explores the "natural human semiotic capacity for image-making [in which] systematic features of sequence arise - that is, a grammar." ${ }^{21}$ While natural languages are appropriate objects of study for such investigations, cultural artifacts constituted of or involving those languages are not, at least not directly. The category of "comics," as a class of cultural artifacts, is "bound to its socio-cultural context, and cannot be extended as a pan-temporal and cross-cultural universal. ${ }^{, 22}$ Comics are manifestations of their cultural contexts and must be investigated as such.

The mistake that many comics scholars make is considering comics and the visual language in which they are written as a singular phenomenon. Partially this is an accident of history. For the past century, in U.S. culture at least, comic books have been the most prominent manifestations of the use of visual languages. The fact that visual language has been relegated to the comics medium ${ }^{23}$ has encouraged the conflation of the medium of comics with the visual languages in which they are written.

I affirm this distinction of Cohn's between "comics" as a category of cultural artifacts and the visual language in which they are written. I take this distinction to be fully compatible with the following investigation of the cultural import and implications of the typical formal features and content of U.S. comics over the past century. The implication of this distinction for comics studies is that one can choose to approach comics in terms of visual languages that comprise them or in terms of their cultural import and meaning.

${ }^{21}$ Cohn, "Un-Defining 'Comics,", 2.

${ }^{22}$ Cohn, 6.

${ }^{23}$ Cohn, 9. It should be noted that Cohn rejects the use of the phrase "comics medium" because he takes "medium" to reference type of "structure," whereas I will use the term to refer to one of many types of culturally relative artistic forms (such as, film, theatre, etc.) and not a biologically anchored behavior like language. 
Cohn writes, "no doubt, there will come a point where the structural investigations of 'visual linguistics' can then give way into literary and social investigation, just as the knowledge of grammatical parts and language groups informs rhetoricians and literary scholars, though it doesn't drive their fields". ${ }^{24}$ The following exploration of relationship of comics' form with examples of their narrative content will be informed by the work of Cohn and others studying the grammar of visual languages, but ultimately will be a philosophical and cultural examination. Now that I have discussed the formal features that are typical of comics and the question of their definition, the discussion turns to the nature of the perceptual and cognitive engagements that readers have with comics.

${ }^{24}$ Cohn, 10. 


\section{I: CLOSURE AS NON-SENSORIAL COGNITION OF DEPICTED COHERENCE}

Imagination, Inference, and Comics

Because of the ways that comics communicate stories, the typical reading experience involves a process that comics scholars have come to call "closure.", 25 Throughout this chapter, I will argue that two particular mental functions are involved in the process of closure: namely, imagination and inference. I will spell these ideas out in greater detail below, but for now it is enough to say that closure is enacted in relation to both small segments of a comics narrative, and also in relation to larger portions of the narrative. As a part of the cognitive process of closure, readers typically imagine spatiotemporal shifts in the storyworld of the comic, resulting in the recognition of a locally coherent storyworld. Readers typically enact this aspect of closure across the gutter between two panels, but they may also enact this type of imagining across small sequences of panels and within individual panels.

Beyond this, when enacting closure, readers make inferences about higher-order and typically narratively relevant conceptual information, resulting in the recognition of a globally coherent storyworld. These inferences typically have propositional content that may or may not be easily represented visually in a narratively relevant way. Both of these

\footnotetext{
${ }^{25}$ McCloud, Understanding Comics, 67.
} 
functions (imagination and inference) are involved in the reader's understanding of a comic as depicting a coherent storyworld.

This distinction between the two mental functions involved in closure, "imagination" and "inference," requires a brief discussion about my particular usage of both terms. Within this work, I am using the term "imagination" to reference what elsewhere has been called, "perception-like" imaginings. ${ }^{26} \mathrm{I}$ am using this term in a way that parallels philosopher Amy Kind's claims that all imagining has some sort of perceptual character. Kind argues, "No matter what I imagine, my imagining will involve an experiential aspect. Without such an experiential aspect, a mental exercise is not an act of imagining. ${ }^{27}$ To imagine something, in this sense, is to imagine something one could experience. Therefore, I take imagination to involve a mental representation of potentially sensory information, which has a phenomenological character, in the absence of a direct confrontation with that sensory information (that is, without a direct confrontation of what is imagined).

Some philosophical accounts of imagination will categorize this type of phenomenological mental activity as only one particular subset of imagination, broadly construed. Imagination, in the sense I am using the term, is sometimes also called "perceptual" or "sensory" imagination. ${ }^{28}$ In regard to closure within comics, a reader imagines (in this perceptual sense) the sensory experience that she (anticipates she)

\footnotetext{
${ }^{26}$ Gregory Currie and Ian Ravenscroft, Recreative Minds: Imagination in Philosophy and Psychology (New York: Oxford University Press, 2003): 11.

27 Amy Kind, "Putting the Image Back in Imagination," Philosophy and Phenomenological Research 62, no. 1 (2001): 94.

${ }^{28}$ Currie and Ravenscroft, Recreative Minds, 12.
} 
would have if she actually found herself perceiving the storyworld of the comic. As Neil Cohn writes:

When we see the world, our vision takes in information from the whole visual array, but we only focus on the parts of that vision that fall within our 'spotlight of attention.' Panels serve a similar function for visual narratives, and thereby can simulate what our vision would be like if we were watching a scene in person. ${ }^{29}$

While I am using the term "imagination" to refer to the type of mental activity with a perceptual character, ${ }^{30}$ closure also involves the process of discerning typically narratively relevant propositional information about the storyworld communicated by the comic in ways that are not perception-like. I refer to the process of recognizing and understanding propositional narrative information as "inference." The use of this term is due primarily to Neil Cohn's prominent use of the same term in his explanations (or reductions, as it were) of the process of closure..$^{31}$ Despite my differences with Cohn (which are spelled out below), I have adopted his terminology to acknowledge the insight his work offers into the conceptual and propositional aspects of the function of closure in reader experience.

It must also be noted that some philosophical accounts of imagination have included something very similar to this inferential element as a subset of imagination. For example, philosophers Gregory Currie and Ian Ravenscroft discuss imagination in terms of both "perceptual imagination" (that is, what I am calling "imagination") and "propositional imagination." 32 What Currie and Ravenscroft call "propositional imagination" is closely related to the process that I am calling "inference" in that both

${ }^{29}$ Cohn, The Visual Language of Comics, 59.

${ }^{30}$ Currie and Ravenscroft, Recreative Minds, 12.

${ }^{31}$ Neil Cohn, "The Limits of Time and Transitions: Challenges to Theories of Sequential Image Comprehension," Studies in Comics 1, no. 1 (2010): 135.

${ }^{32}$ Currie and Ravenscroft, Recreative Minds, 94. 
involve the discerning and entertaining of propositional and conceptual (rather than potentially perceptual or sensory) information regarding, in this case, the storyworld of the comic.

Currie and Ravenscroft write that, "the concept of a state of propositional imagining is the concept of a state from which we reason in the same ways as from beliefs and desires. And 'reasoning in the same way from' means reasoning from the same premises to the same conclusions by the same inferential route." "33 "Inference," as I am calling it, involves what Currie and Ravenscroft refer to as "belief-like imagining," rather than "desire-like imagining." " That is, inference involves supposing certain propositional information without taking a particular perspective toward that information, as would be the case with phenomenological and perceptual imaginings. ${ }^{35}$

I do not take the views expressed here to conflict in any significant way with the distinction articulated above, other than in terms of verbiage. ${ }^{36}$ When discussing the experience of reading a Superman comic, it is entirely appropriate to say that the reader is imagining that Superman lives in Metropolis (that is, a propositional fact about the storyworld of the comic). This locution of "imagining that" is simply using the term "imagining" in a more inclusive (and more vernacular) sense than I am using it within this work to discuss the mental processes associated with closure. Again, by "imagining"

\footnotetext{
${ }^{33}$ Currie and Ravenscroft, Recreative Minds, 94.

${ }^{34}$ Currie and Ravenscroft, 34.

${ }^{35}$ Currie and Ravenscroft, 33-38.

${ }^{36}$ Kind, "Putting the Image Back in Imagination," 87. I am not, for example, interested here in arguing for or against Kind's "imagery model" of imagination, which holds that the use of the term "imagination" in all contexts necessarily indicates a phenomenological/perceptual/experiential element. I am only here concerned with clarifying the use of the term "imagination" when explaining the process of closure in the context of comics.
} 
I will mean the process of a reader mentally simulating perceptual experiences, while by "inferring" I mean the process of a reader actively extrapolating conceptual and propositional content. Both of these cognitive functions serve to constitute the process of closure.

Closure: Notable Approaches

The term "closure" was used first in relation to Gestalt psychology by Max Wertheimer in 1923 to reference the ability of the human mind to perceive whole objects when presented with only partial visual access to said objects. ${ }^{37}$ In other words, one perceives the entirety of a shape, numerical symbol, or other familiar pattern even when the available visual information is incomplete or part of the pattern is absent. This phenomenon does not appear only in the Gestalt psychological literature, however. Phenomenologists have discussed a similar capacity for aspects of our environment to be present for us without being fully included in our visual experiences. ${ }^{38}$

McCloud, in Understanding Comics, appropriated the term to refer to readers' perception of and engagement with sequential images in comics and other graphic narratives. McCloud writes that closure is "the phenomenon of observing the parts but perceiving the whole. ${ }^{39}$ His notion of closure refers in part to the imaginative engagement that readers of comics enact when they encounter gutters. ${ }^{40}$ While the panels present the content of the sequence, the gutters present the breaks in and the structuring

\footnotetext{
${ }^{37}$ Max Wertheimer, "Laws of Organization in Perceptual Forms," Classics in the History of Psychology, accessed on March 13, 2016, http://psychclassics.yorku.ca/Wertheimer/Forms/forms.htm.

38 Joel Smith, "Seeing Other People," Philosophy and Phenomenological Research LXXXI, no. 3 (2010): 735-39.

${ }^{39}$ McCloud, Understanding Comics, 63.

${ }^{40}$ McCloud, 63-66.
} 
of that content. McCloud writes, "here in the limbo of the gutter, human imagination takes two separate images and transforms them into a single idea. Nothing is seen between the two panels, but experience tells you something must be there!"41 Here, McCloud draws on the role that a reader's expectations play in understanding the storyworld of comics. As I will discuss below, readers do not simply imagine and infer anything in the gaps left by the gutter, but content that will allow the storyworld of the comic to be understood as coherent.

Throughout Understanding Comics, McCloud draws parallels between the processes of closure and the notion of object permanence (that is, the perception that objects continue to exist even when they are not directly observed). He draws on the developmental psychological models of Jean Piaget when explaining closure, writing, "the game 'peek-a-boo' plays on this idea." ${ }^{42}$ When the adult covers his or her face, the child initially may react with confusion, as if the adult's face is simply gone. However, McCloud writes, "gradually, we all learn that even though the sight of mommy comes and goes, mommy remains." ${ }^{43}$ McCloud claims that this same cognitive process occurs when we engage with comics. Specifically, comics often present readers with images in which portions of objects (or the entirety of objects) appear to be occluded and yet readers understand that those objects are not being depicted as partial. ${ }^{44}$

\footnotetext{
${ }^{41}$ McCloud, 66-67.

${ }^{42}$ McCloud, 62.

${ }^{43}$ McCloud, 62.

${ }^{44}$ Groensteen, The System of Comics, 40. Of course, they are not actually occluded, because the depictions in comics constitute all the visual information available. "To close the panel is not to stop the drawing. The graphic materiality cannot flee or flow out; no need, then, to limit it through coercive means. To close the panel is to enclose a fragment of space-time belonging to the diegesis, to signify the coherence."
} 
The process of closure, as McCloud understands it, also involves inference when readers "mentally [complete] that which is incomplete based on past experience." ${ }^{45} \mathrm{He}$ understands closure as constituting a large part of what is unique about the medium of comics and as the primary method of understanding involved in engaging with sequential images. He writes, "If visual iconography is the vocabulary of comics, closure is its grammar. And since our definition of comics hinges on the arrangement of elements -- -then, in a very real sense comics is closure!" ${ }^{\prime 46}$ Since the writing of McCloud's influential work, however, elements of his view of closure have been challenged.

In his influential work The System of Comics, semiotic theorist Thierry Groensteen deals very little with McCloud's use of the term "closure." In fact, Groensteen uses the term for his own purposes to refer to a particular function of the borders of comics panels, namely, enclosing them. ${ }^{47}$ But the fact that he does not address the term "closure" as McCloud uses it does not preclude Groensteen from addressing the processes to which McCloud's term refers. Groensteen focuses heavily on the structural elements of comics, even introducing a unique taxonomy of those elements to discuss what he calls "arthrology," the process by which various levels of meaning are communicated through the micro and macro structures of the comics form. ${ }^{48}$

In articulating the various levels of understanding and interpretation involved in arthrology, Groensteen discusses the roles of various formal features of comics. For

\footnotetext{
${ }^{45}$ McCloud, Understanding Comics, 63.

${ }^{46} \mathrm{McCloud}, 67$.

${ }^{47}$ Groensteen, The System of Comics, 40-43.

${ }^{48}$ Groensteen, The System of Comics, ix. In Groensteen's terms, "restrained arthrology" and "general arthrology."
} 
example, he writes that the gutter represents "a forced virtual, an identifiable absence" 49 in the content of the sequence of images. "The gutter is simply the site of this absence. More than a zone on the paper, it is the interior screen on which every reader projects the missing image (or images). ${ }^{, 50}$ In saying this, he affirms McCloud's position that gutters are sites that necessitate reader imagination. Additionally, Groensteen points out that the use of speech balloons, thought bubbles, and caption boxes also imply the necessity for closure within a panel. Because these elements typically appear within panels and therefore appear to "block" portions of the depiction contained in the panel, readers must enact closure in order to fully understand the content "behind the zone hidden by the balloon." 51 This element of closure resembles the Gestalt psychological notion although the scope of implications of closure in regard to comics differs, as is discussed below. ${ }^{52}$

Groensteen gestures toward a concept similar to the one I will articulate below in terms of "coherence," as well. He writes, "one cannot conceptualize comics without verifying the general rule, that of iconic solidarity. The necessary, if not sufficient, condition required to speak of comics is that the images will be multiple and correlated in some fashion." ${ }^{, 53}$ Groensteen's reference to the correlation of images is distinct from the notion of "coherence" that I will outline below, but it is clearly a similar idea in that it gestures toward the relatedness that is usually found across the presentation of various panels. The distinction consists in that Groensteen's notion of iconic solidarity primarily

${ }^{49}$ Groensteen, 113 .

${ }^{50}$ Groensteen, 113 .

${ }^{51}$ Groensteen, 71.

${ }^{52}$ McCloud implies that this particular mental function is included within his understanding of closure by adopting the term from Gestalt psychology, as well as by including panels that depict occulted, but familiar, objects.

${ }^{53}$ Groensteen, The System of Comics, 19. 
references visual and aesthetic similarity across comics pages (that is, across multiple panels). ${ }^{54}$ The notion of "coherence" that I will articulate below emphasizes readers' experience of the nature of the content of the storyworld presented by comics, rather than only addressing the similarity of depictions, or readers' actual perceptual experiences.

While McCloud and Groensteen focus primarily on the gutters between juxtaposed panels, other comics scholars have offered alternative perspectives on where closure occurs. Neil Cohn offers a critique of and an attempt to refine McCloud's conception of closure. Cohn argues that McCloud focuses too heavily on the gutter as playing an active role in delivering the content of sequences of images. ${ }^{55}$ Cohn argues, "the gap [of the gutter] cannot be filled [via closure] unless it has already been passed over, making closure an additive inference that occurs at panels, not between them." ${ }^{, 56}$ Cohn also argues that McCloud ignores the broader "narrative grammar" of sequences of images,${ }^{57}$ even calling McCloud's description “in part, just rhetorical hand waving. Ultimately, Cohn reduces the notion of closure to the process of inferring conceptual information about the storyworld that is implied by the content of the panels. ${ }^{59}$

Going forward, I will demonstrate how the notion of closure with regard to comics (as a category of cultural artifact) can be further detailed. I will delineate the common ground between the cross-cultural structuralist approach of Cohn and more

\footnotetext{
${ }^{54}$ Groensteen, The System of Comics, 19. He writes, "[Focusing on panel-to-panel] reading does not take a lesser account of the totality of the panoptic field that constitutes the page (or the double page), since the focal vision never ceases to be enriched by peripheral vision."

${ }^{55}$ McCloud, Understanding Comics, 66. McCloud writes, "in the limbo of the gutter, human imagination takes two separate images and transforms them into a single idea." ${ }^{56}$ Cohn, "The Limits of Time and Transitions," 135.

${ }^{57}$ Cohn, The Visual Language of Comics, 65-89.

${ }^{58}$ Cohn, "The Limits of Time and Transitions," 135.

${ }^{59}$ Cohn, The Visual Language of Comics, 217.
} 
culturally sensitive approaches, such as the work of McCloud and Groensteen. In doing so, it will be helpful to explore examples of sequences of images in comics in which closure obtains and examples in which closure does not.

\section{What Closure is Not}

Below, I will offer a detailed description of the function of closure in readers engagements with comics. But first, in order to determine how closure should best be understood, especially in light of the brevity of McCloud's initial discussion of the concept and disagreements about it since, it will be useful to rule out possible functions that closure does not serve before attempting to offer a positive account of its functional boundaries. There are aspects of both the content and structure of sequences of images that can be ruled out with regard to how readers may enact closure when engaging with comics. I will consider closure's relation to content and structure in that order.

First, it is worth noting that closure with regard to the content of sequential images is not only an application of the Gestalt psychological notion of the principle of closure. While McCloud appropriated the term, the function described is not identical because closure with regard to graphic narratives does not only occur when the representations are missing visual information that would usually be present. And it can apply across gutters in the sequence of images, which is not an aspect of the Gestalt notion but it is this feature of closure that McCloud emphasizes as primary.

By viewing two panels that depict the same character engaged in an action at two distinct points in narrative time, the reader can enact closure. In cases like this, direct depictions of the temporal aspects of the narrative (rather than only visual information) are technically absent because comics are constituted of static, rather than moving, 
images. This aspect clearly distinguishes closure in comics from the Gestalt psychological notion. McCloud discusses this temporal dimension of closure, writing that time and space are represented in a singular (visual) modality in comics. ${ }^{60}$

However, closure does not necessarily imply temporal linearity within a comic. Consider Alison Bechdel's Fun Home: A Family Tragicomic, in which Bechdel recounts important events in her life and her relationship with her father by depicting scenes from her childhood and young adulthood in non-chronological order. Bechdel leaps from representations of her mid-twenties to her childhood and back in an order that serves her narrative purposes. So, while closure in comics can imply movement forward in narrative time, temporal linearity is not necessary to the function of closure. Rather, narrative temporal shifts are often a feature of images in comics that allow readers to enact closure.

In addition to closure's potential lack of temporal linearity, Neil Cohn argues that depictions within panels need not suggest any specific amount of narrative time. He cites examples of "polymorphic" comics panels, which are "not attached to any sense of spatial [reading] progression [within the panel]. ${ }^{, 61}$ The panel below (Figure 1) depicts a dog chasing his tail, but for how long? Regardless of the actual time it takes for the reader to engage with and comprehend the contents of the panel, the amount of narrative time represented remains somewhat ambiguous. The panel just "conveys the durative concept of a dog running in circles, ${ }^{, 62}$ rather than visually definitively representing the temporal element of the event. This point of Cohn's is well taken, but needs to be further refined.

\footnotetext{
${ }^{60}$ McCloud, Understanding Comics, 100.

${ }^{61}$ Cohn, "The Limits of Time and Transitions," 132.

${ }^{62}$ Cohn, 133.
} 
While polymorphic panels may not convey a highly specific amount of narrative time, such panels must fall within some range of appropriate interpretations with regard to the length of narrative time that the image conveys. For example, while the panel below can be interpreted as lasting anywhere from a few seconds to over a minute, attempting to read the panel as representing multiple hours, days, or years of narrative time is untenable. The shadows of both the human and the dog suggest a roughly stationary position relative to the sun and the inclusion of a speech balloon communicating that the human character laughs show that polymorphic panels can communicate information regarding the amount of narrative time that they depict, even while not determining it to a highly specific degree.

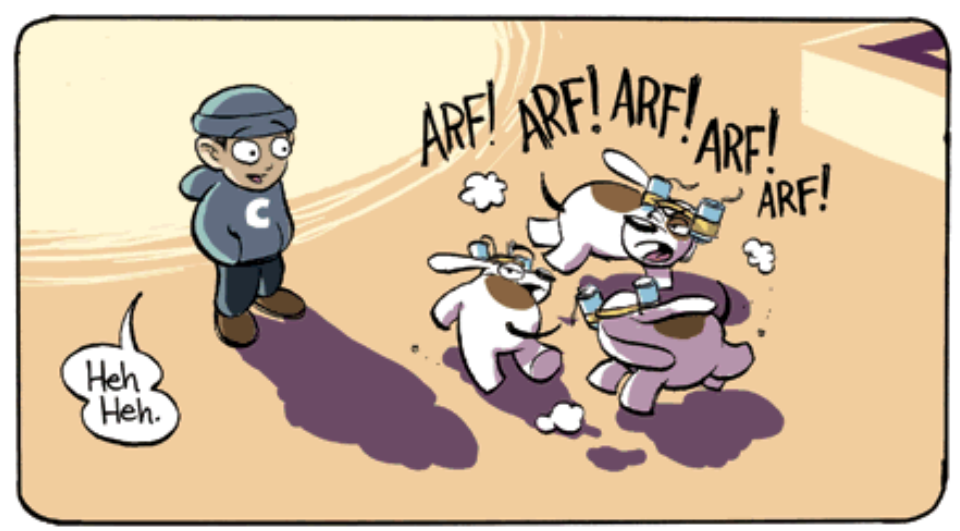

Figure 1: Kazu Kibuishi, Copper (New York: Graphix, 2010), reprinted in Cohn, "The Limits of Time and Transitions," 132.

McCloud, in Understanding Comics, offers a detailed discussion of the relation of narrative time to the content of panels. Cohn's discussion is a direct response to McCloud's on this topic. McCloud draws attention to the role of speech or other sounds within panels as influencing the range of appropriate interpretations of the duration of narrative time that is being depicted. To exemplify this, he offers an elongated panel depicting a period of narrative time in order to make the point that even a single panel 
can represent more than a single moment in a story. ${ }^{63}$ Because readers attend to different portions of such a complex panel across different moments of actual time, a single panel can (but does not necessarily) communicate multiple moments of narrative time. Therefore, the actual spatial dimension of the panel on the page can influence a reader's understanding of the narrative duration communicated by the image.

McCloud then introduces various speech balloons into the same panel. In doing so, he does not change the depiction ${ }^{64}$ but he does change the events depicted. ${ }^{65}$ The dialogue firmly establishes that various characters are reacting to other characters depicted within the same panel, which tells us that more than one moment is being represented. So, while Cohn is right that McCloud is overly reliant on the identification of page space with narrative time,${ }^{66}$ Cohn's claim that polymorphic panels simply convey the concept of the type of motion depicted ignores the fact that such depictions suggest an acceptable range of interpretations with regard to the amount of narrative time they are communicating. The enactment of closure is not dependent upon a highly specific amount of depicted narrative time, and panels cannot be understood as necessarily representing single moments of narrative time.

Closure in relation to comics does not rely on depictions of content that take a robustly narrative form, either. A clear example of closure in the absence of full narrative

${ }^{63}$ McCloud, Understanding Comics, 96.

${ }^{64}$ Technically the depiction does change, but only in that elements of the background that were previously visible are obscured with word balloons.

${ }^{65}$ McCloud, Understanding Comics, 97.

${ }^{66}$ Cohn, "The Limits of Time and Transitions," 131. "The time it takes to read something and the mental abstraction of time within the fictitious narrative are not comparable, and exist on totally different levels of analysis and experience. This is why fictive time is unaffected by different arrangements of the same panels in varying layouts, though layout might affect the physical rhythmic pace in which those panels are read." 
is a two-panel sequence in which the reader sees two moments across one event. The depiction of a single event (such as the figure taking off his top-hat in Figure 2) does not present a fully narrative structure ${ }^{67}$ or meet what Groensteen calls the "threshold of narrativity, ${ }^{, 68}$ but presents a single event in isolation. No broader meaning or significance is conferred to the event's depiction because the event is not part of a fully narrative or story structure.

One potential objection to this claim is that such a minimal structure would qualify only as a comic strip, rather than as a comic book or graphic novel. While it is true that absent a larger narrative structure (and the inclusion of more images) such a juxtaposition of images only constitutes a comic strip, the perceptual engagement of the reader is the same across two such panels as it would be across the same panels if they were embedded in a longer sequence. Two panels that communicate one event, failing to constitute a narrative structure,${ }^{69}$ reveal that closure can occur in the absence of fully narrative content. These two panels contain no story. One could attempt to counter this claim by arguing that the depiction of any single event constitutes a narrative. But this could also be used to argue that individual images constitute narratives, which is counterintuitive. $^{70}$

${ }^{67}$ Peter Goldie, The Mess Inside: Narrative, Emotion, and the Mind (Oxford, UK: Oxford University Press, 2014): 6, 11. ${ }^{68}$ Groensteen, The System of Comics, 104.

${ }^{69}$ Goldie, The Mess Inside, 10-11. Here, I am affirming philosopher Peter Goldie's view that the process of "emplotment" is needed to "[mediate] between the raw material and the narrative itself." The "raw material," here, refers to actual events in the world or bare representations that lack the process of "shaping, organizing, and colouring the raw material into a narrative structure."

${ }^{70}$ Goldie, The Mess Inside, 6. "A narrative is more than just a bare annal or chronicle or list of a sequence of events." 


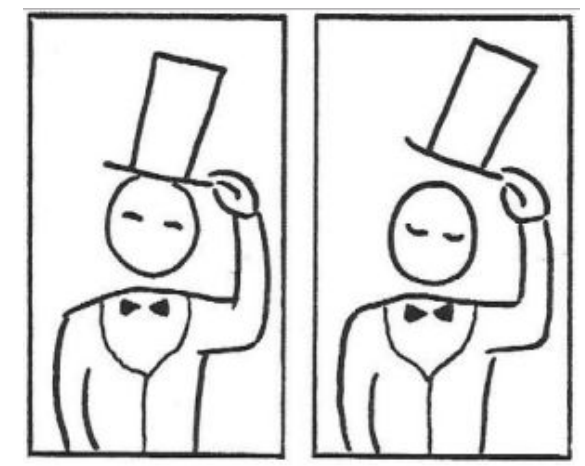

Figure 2: McCloud, Understanding Comics, 65.

It is useful here to reemphasize Cohn's distinction between comics and the visual language in which comics are written. It may be that some minimal amount of "visual narrative grammar" ${ }^{, 1}$ is necessary in order for readers to enact closure on a sequence of images. However, Cohn's notion of "visual narrative grammar" does not utilize the term "narrative" in its intuitive sense-that is, a story. In the same way, it would not be the case that any strings of words that have both semantic content and an analyzable syntax necessarily represent a narrative in any intuitive sense. However, this is not a problem for Cohn's account, as he is primarily focused on the natural language in which comics are written, and not comics in their context as cultural artifacts.

Philosopher Peter Goldie illuminates the requirements for a narrative structure to obtain. While he focuses on more traditional narrative media, I will extend his analysis to comics. Goldie cites coherence, meaningfulness, and evaluative and emotional import. Coherence, according to Goldie, amounts to the notion that narrative structures reveal, "through the process of emplotment, connections between the related events $[\ldots]$ in a way that a mere list, or annal, or chronicle, does not."72 So, narratives must include multiple events and their coherence is determined by how the events "hold together in

${ }^{71}$ Cohn, The Visual Language of Comics, 69.

${ }^{72}$ Goldie, The Mess Inside, 14. 
some way" and narratives usually "appeal to the [causal] elements that are relevant to [...] the interests of [their] audience."73 Goldie's use of the term "coherence" is useful as a requirement in articulating the conditions for fully narrative structures; however, my use of the term "coherence" in explicating closure refers to a slightly different condition for how multiple depictions (across panels, pages, etc.) hold together, namely that they represent a single (though potentially multifaceted) storyworld.

The second of Goldie's conditions for narrative structure is meaningfulness. The meaningfulness of a narrative must be understood in relation to a particular perspective. In other words, for a narrative to be meaningful it must be meaningful to someone. Goldie offers two types of perspectives from which a narrative can be meaningful. Narratives may have meaning with regard to the perspective of those people who are internal to the narrative (e.g., characters). He calls this the "internal perspective.",74 Secondly, narratives may have meaning from the "narrator's external perspective." This perspective may reveal the narrator's explicit intentions in relaying the story or it may reveal (whether intentionally or unintentionally) "aspects of the narrator's personality.,"75 Typically, a narrative will express both of these types of meaningfulness simultaneously. ${ }^{76}$

Finally, Goldie argues that narrative structures necessitate evaluative and emotional import. He writes that this idea "is really very simple. Things matter to people,

\footnotetext{
${ }^{73}$ Goldie, 14, 16.

${ }^{74}$ Goldie, 17.

${ }^{75}$ Goldie, 33.

${ }^{76}$ Goldie, 34. "Appreciation of the perspectives of those who are internal to the narrative, and appreciation of the external perspective of the narrator, are bound up with each other in highly complicated ways."
} 
and a narrative involving people can capture the way things matter to them." ${ }^{, 77}$ Like the criteria of meaningfulness, evaluative and emotional import presumes perspectives and can manifest either internally (through the emotional/evaluative interests of people within a narrative) or externally (through the emotional/evaluative interests of the narrator). I will explore the conception of narrative (especially in regard to conceptions of selfhood) later, but this brief look should serve to solidify that a fully narrative structure is not a requirement in order for a sequence of images to qualify as an opportunity for readers to enact closure, regardless of Cohn's more narrow use of the term "narrative."

Also, closure does not entail that direct depictions of events are necessary in order to communicate events as a part of the content of a sequence of images. For example, an event can be implied by a sequence without being directly depicted within any of the panels. $^{78}$ So, the explicit depiction of an object or event within an included panel is not necessary in order for the closure enacted by the reader to include that object or event as part of the content of the storyworld depicted by the sequence. McCloud's example of the axe murder (Figure 3 ) highlights the role that inference can play. ${ }^{79}$ The first panel in this sequence features an image of an angry looking man shouting, "now you die!!" while swinging an axe at another character who yells, "no! no!" The second panel features an image of a skyline beneath the interjection, "EEYAA!!" 80 In this example, readers infer propositional content implied by the sequence, namely, that the character with the axe murdered the other character. However, closure does not simply reduce to inference, as

\footnotetext{
${ }^{77}$ Goldie, 22.

${ }^{78}$ See Cohn, The Visual Language of Comics, 112-115 and McCloud, Understanding Comics, 69.

${ }^{79}$ McCloud, Understanding Comics, 66.

${ }^{80} \mathrm{McCloud}, 66$.
} 
Cohn seems to believe, as this would suggest a conscious conceptual component that need not be present in the reader's experience. I do not do conscious conceptual work to infer that the two panels above (Figure 2) depict one human tipping their hat, but rather I simply imagine it.
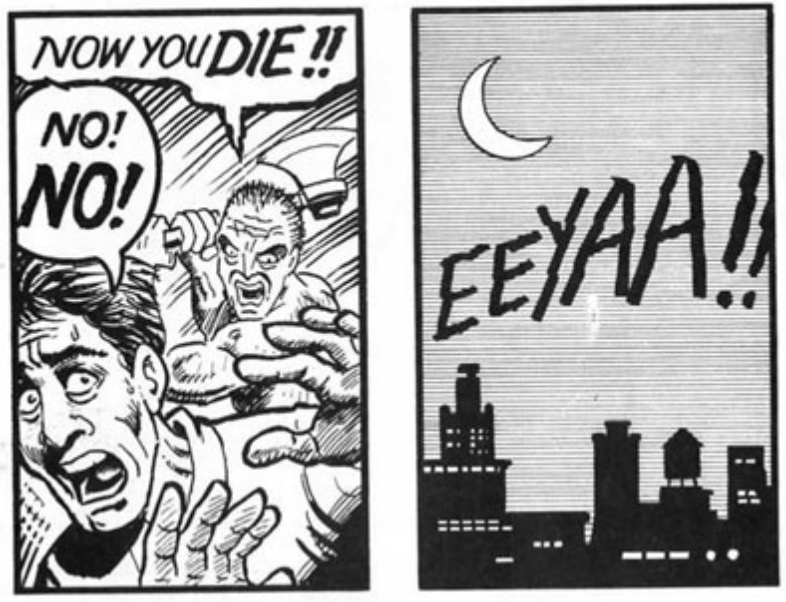

Figure 3: McCloud, Understanding Comics, 66.

The closure enacted in a set of sequential images is not constrained to represent only one unified storyworld. In cases such as the "Tales From the Black Freighter" comic within the narrative of Watchmen, depicted storyworlds can be nested within one another. ${ }^{81}$ Readers enact closure with regard to the events depicted by the "Black Freighter" storyline, even though that storyworld is within a comic book being read by one of the characters in the storyworld depicted in Watchmen. So closure can also occur in nested sequences, taking place in storyworlds depicted within storyworlds.

Another interesting application of the notion of closure involves a depicted character addressing the actual world (that is, the world of the reader), also known as "breaking the fourth wall." This phrase initially referred to the imaginary wall between the theatre stage and the audience, and therefore between the storyworld and the actual

${ }^{81}$ Alan Moore and Dave Gibbons, Watchmen (New York: DC Comics, 1986-1987). 
world. Breaking the fourth wall complicates the closure of the storyworld of the comic by referencing the actual world to some degree. Consider Marvel's sarcastic antihero Deadpool, who routinely addresses the reader directly and mentions the artists and writers who are responsible for the book in which he is depicted. He also discusses the fact that he is a comic book character and acknowledges the visual conventions of the medium that are not internal to the storyworld, including caption boxes and speech balloons. $^{82}$

This type of closure between the actual world and the storyworld presented by comics that break the fourth wall is a significantly different experience for the reader than the nesting of worlds within worlds, as in Watchmen's "Tales From the Black Freighter." Because they involve themselves in the reader's world, sequences that enact this type of closure toy with the typical metaphysical gap between fictional worlds (or storyworlds more broadly) and the actual world. ${ }^{83}$

Another example of this breaking of the fourth wall appears in DC's Harley Quinn issue \#0, entitled "Picky Sicky," in which different artists draw the character of Harley on different pages of the issue. This issue breaks the fourth wall in that Harley is depicted as being aware of these artistic transitions as they occur and the voices of the creative teams are included in stylized speech balloons so that the character of Harley can discuss the pros and cons of each artist with the artists and writers. Ultimately, Harley

82 “ $4^{\text {th }}$ Wall Awareness," SuperpowerWiki, accessed February 19, 2017, http://powerlisting.wikia.com/wiki/4th_Wall_Awareness.

${ }^{83}$ Kendal Walton, "Fearing Fictionally," in Arguing About Art: Contemporary Philosophical Debates, ed. by Alex Neill \& Aaron Ridley (New York: Routledge, 2008): 257. Within debates regarding the philosophy of fiction, it is taken as intuitive that there is a "gulf separating fictional worlds physically from the real world" that is "unbridgeable" and makes it difficult to understand "psychological interaction across worlds." 
"decides" that Chad Hardin (the regularly appearing artist on the book) is best. On the penultimate page, after surviving an explosion, Harley finds a page of a comic book among the debris. The page she finds features Hardin's artwork because it is the final page of "Picky Sicky" (that is, the page following the page on which it is seen by Harley) and she responds to Hardin's art, saying, 'Hey, now! This is what I'm talkin' about! Look how nice this guy's art is. I wonder if he can keep a monthly schedule? With my luck, he'll probably need a fill-in artist by issue two." ${ }^{, 84}$

Finally, with regard to the content of sequences, closure does not require a definite interpretation of content or meaning. In other words, readers can enact closure with regard to a sequence of images without taking a single definite interpretive stance toward the events being represented. Consider the final page of Alan Moore's Batman: The Killing Joke (Figure 4). The Joker has just told Batman a joke after enacting a long streak of sadism and brutality toward those to whom Batman is close. The final panels depict the silhouettes of Batman and the Joker. Batman's arms are reaching across the divide between them, making contact with the Joker and both appear to be laughing. The bottom panels of the page depict rain falling on the ground and the laughter stops in the final three panels.

Recently, comic book author Grant Morrison (who was not involved in the creation of the book) stated that he interprets the end of the story as portraying Batman murdering the Joker. Morrison stated, “That's why it's called The Killing Joke. The Joker tells the 'Killing Joke' at the end, Batman reaches out and breaks his neck, and that's why

\footnotetext{
${ }^{84}$ Amanda Conner, Jimmy Palmiotti, and Chad Hardin, Harley Quinn Volume 1: Hot in the City (New York: DC Comics, 2014).
} 
the laughter stops and the light goes out.. ${ }^{85}$ While some fans disagreed with Morrison's interpretation, preferring the standard interpretation that Batman simply is laughing before taking the Joker back into captivity, this example shows that closure need not hinge on unambiguous content within a sequence.

It could be argued that closure is simply enacted differently by the two groups of readership (i.e., those who adhere to Morrison's interpretation and those who adhere to the standard interpretation). On this view, the two groups then disagree about which account of closure is more accurate and these are competing and mutually exclusive enactments of closure. One might argue that this entails that closure requires a determinate narrative interpretation of some sort. However, this would ignore the possibility that a reader might be undecided on what they take the most likely interpretation of a particular sequence to be. For example, readers may be able to shift between reading The Killing Joke with Morrison's interpretation and with the standard interpretation. Therefore, a sequence can contain ambiguous content and still be a possible site of closure. With all these comments about the relation of closure to the content of a sequence concluded, I turn to the relation between closure and the structure of a sequence.

${ }^{85}$ Chris Begley, "Grant Morrison: Batman kills Joker in 'The Killing Joke,"” Batman News, August $16^{\text {th }}, 2013$, http://batman-news.com/2013/08/16/grant-morrison-batmankills-joker-in-the-killing-joke. 

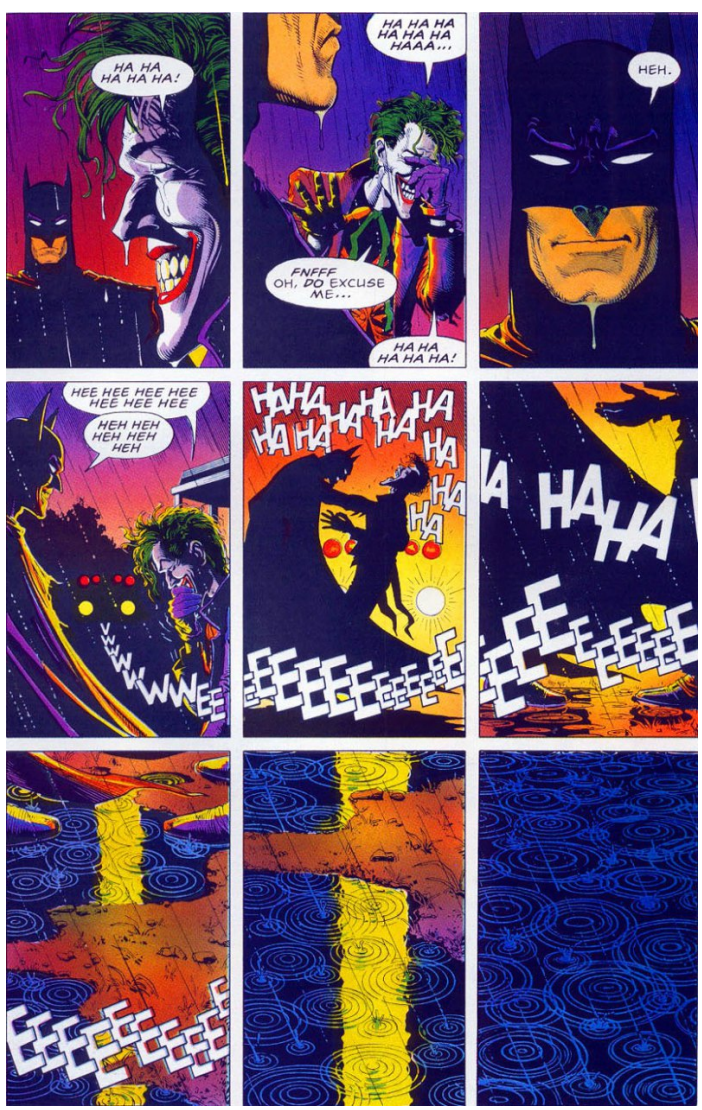

Figure 4: Alan Moore and Brian Bolland,

Batman: The Killing Joke (New York: DC Comics, 1988).

There seem to be few structural requirements for closure to take place when a reader engages with a comic. For example, closure is not achieved through the visual artistic or textual modalities directly. That is, closure is not a sensorial or perceptual process, simply. This is practically definitional for the concept of closure in regard to comics. It is precisely the cognitive processes that result in the understanding (in a certain way or toward a certain end $)^{86}$ of what is absent to one's sensory modalities that constitutes closure. So while in the case of comics visual observation is necessary for closure to occur (that is, a reader must see the sequence of images), the enactment of

\footnotetext{
${ }^{86}$ Perceiving toward the end of the coherence of the depicted storyworld of the sequence, specifically.
} 
closure is not realized in the visual field or perceptual capacities of the reader. Closure is enacted when we imagine and infer, not simply when we see and read.

Additionally, closure is not dependent on the consistency or inconsistency of artistic or aesthetic style or medium. A comic book can still be viewed as presenting a coherent storyworld even if the visual artistic style changes from panel to panel or book to book. Representations of characters' changing perceptions or mental circumstances may be communicated with shifts in artistic style without confusing readers. ${ }^{87}$ For example, The Fade Out \#1 uses a sketchy colorless background to represent a character's inattention to his immediate surroundings. This sketchy style breaks from the typically more realistic style of Sean Phillips, the book's artist. Shifts in artistic style also occur without undermining closure when ongoing serialized comic book titles change writers, artists, or both without the implication of representing a different storyworld. Batman's storyworld remains coherent, even when a new creative team is brought onto the title.

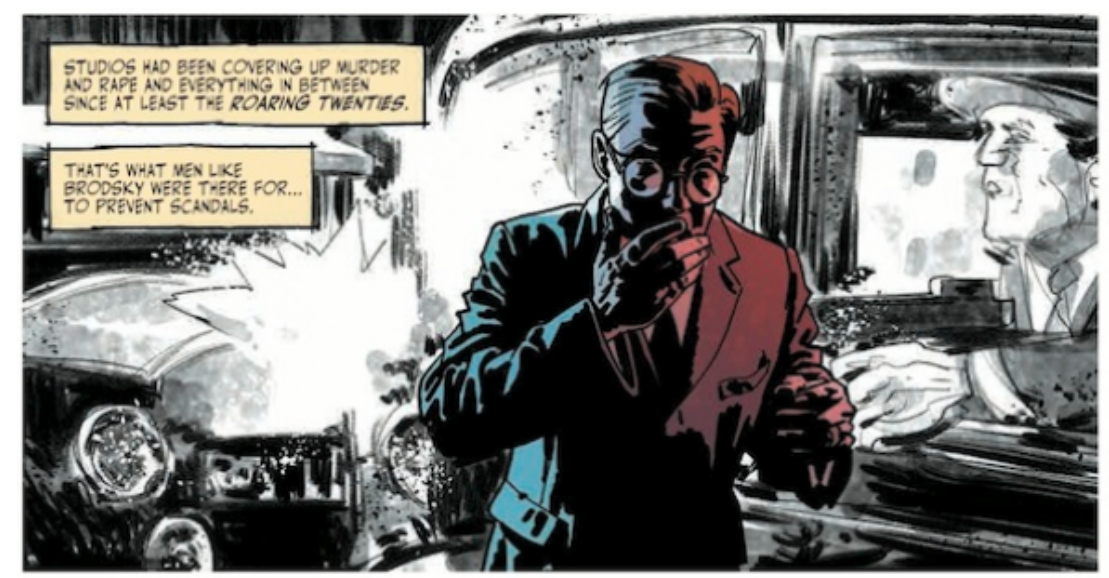

Figure 5: Ed Brubaker and Sean Phillips, The Fade Out: Act One (Portland, OR: Image Comics, 2015).

The physical distance between panels, sequences, or books through which closure can potentially take place is not easily determined. While the gutter between juxtaposing

${ }^{87}$ Duncan and Smith, The Power of Comics: History, Form \& Culture, 159-163. Duncan and Smith classify such artistic strategies as "hermeneutic images." 
panels provides the paradigmatic occasion for closure,${ }^{88}$ closure also may be enacted across sequences of panels,${ }^{89}$ and the inferential aspect of closure may be enacted across entire books. ${ }^{90}$ For example, a reader can understand The Fade Out $\# 4$ as continuing the same series of events in the same storyworld as The Fade Out \#3. Readers may enact the inferential element of closure not only across issues of a continuing series, but also across different serialized titles released by a single publisher. Consider the fictional universes of Marvel comics, in which characters who appear in their own serialized titles (such as, Spider-Man, Captain America, etc.) also appear in other titles in ways that imply a coherence of narrative and storyworld. ${ }^{91}$ The imaginative element of closure is more constrained and both the imaginative and inferential elements are typically present when readers enact closure within comics.

It is doubtful that significant gaps in actual time will typically occasion the imaginative element of closure. For example, if a reader must wait a month (the typical single issue comics release schedule) after reading The Fade Out \#3 before she reads The Fade Out \#4, it may be difficult to enact closure without re-reading issue \#3. Rather, she may simply remember plot points of the narrative of the previous issue (allowing for

${ }^{88}$ Scott McCloud, Understanding Comics, 68.

${ }^{89}$ Cohn, The Visual Language of Comics, 66-67.

${ }^{90}$ The inferential aspect of closure obviously is not unique to comics, in and of itself. Engaging with other kinds of narrative mediums may allow readers/viewers to infer narratively relevant propositional and conceptual information about the storyworld presented (e.g., novels, films, theatre). The distinction with regard to comics is the role that perception plays within the presentation of those opportunities for inference, namely, the place of static visual artwork in the perceptual field of the reader. A reader may infer information from the content of the art within the panels, textual elements, or from the style or structure of the comics page.

${ }^{91}$ However, enactment of closure will be temporally constrained to some extent and will rely on the memory of the reader with regard to works she has previously read and information that is accessible to her, upon which she can make inferences. 
propositional inference). Since closure is a non-sensorial cognitive process paradigmatically enacted across the small gap of gutters between panels, it will be less likely to occur when the reader experiences larger gaps in her engagement with the storyworld being depicted, especially with regard to the imaginative aspect of closure.

When reading The Fade Out: Act One, which collects issues \#1-4 in a single book, a reader will be more likely to enact closure between any two issues than she will when reading them in single issue format with a month of actual time passing between her engagement with them. Without acknowledging the temporally constrained nature of the possibility of enacting closure, it would be far too expansive a concept and lose the focus on the role of perception (that is, seeing the content and structure of and gaps between sequential images). If this were the case, readers could be said to experience a coherent storyworld presented across disparate mediums even if they only feature similar characters or locations (e.g., closure from Batman comic books to Batman movies). While readers are able to understand that TV and comics may represent a single storyworld (for example, comic book prequels and tie-ins to movies are increasingly common), those who engage with such multimedia representations of storyworlds do not experience them in the same way, and therefore these examples don't constitute closure in the sense discussed here.

As I discussed above, the fact that closure can extend beyond immediately juxtaposed panels does not entail that closure is totally unconstrained. In The System of Comics, Thierry Groensteen argues that all panels in a comics work are potentially related and interdependent. ${ }^{92}$ Groensteen writes, "within the paged multiframe that

${ }^{92}$ Groensteen, The System of Comics, 146. 
constitutes a complete comic, every panel exists, potentially if not actually, in relation with each of the others." 93 Groensteen's taxonomy (termed "arthrology") of the levels of meaning that arise for readers of comics via the variously broad structures of the comics form (e.g., panel, page, book, etc.). ${ }^{94}$

Cohn disagrees, arguing for the necessity of a determinate visual language structure (that is, a grammar) in communicating the meaning of a sequence of images. ${ }^{95}$ Cohn's critique of Groensteen is resolvable simply by acknowledging, again, the distinction between comics and the visual languages in which they are written. Groensteen's arthrology is geared toward comics as artifacts that manifest culture, ${ }^{96}$ while Cohn is primarily concerned with the artistic content and structures of sequences as they manifest visual language.

Finally, occasions of closure are not dependent on either external compositional structures or the presence of traditional gutters. The reading order, shape, and potential narrative function of panels do not, on their own, determine whether or not closure may be enacted when readers engage with a given sequence. ${ }^{97}$ While the presence of gutters (that is, blank spaces between juxtaposed panels) offers the clearest example of an opportunity for closure, explicit gutters are not necessary. ${ }^{98}$ For example, multiple speech balloons within a single panel can communicate the passage of time, which entails

\footnotetext{
93 Groensteen, The System of Comics, 146.

${ }^{94}$ Groensteen, 27.

${ }^{95}$ Cohn, The Visual Language of Comics, 66-67.

${ }^{96}$ This is not to suggest that I agree with the content of Groensteen's theory of comics generally, but only to say that it would be a mistake to dismiss such work as inadequate for studying natural languages because Groensteen's analysis is aimed at comics as a cultural artifact.

${ }^{97}$ Cohn, The Visual Language of Comics, Chapter 5.

${ }^{98}$ Groensteen, The System of Comics, 113.
} 
enactment of closure. A single panel, then, can be appropriately interpreted as depicting several narrative moments within the storyworld, requiring the cognitive processes that result in the recognition of coherence on the part of the reader. ${ }^{99}$

In the previous section, I have narrowed the potential tenable understandings of closure by offering many functions of the reading experience and features of comics that are not necessary for closure. The closure we enact when we read a comic is distinct from the Gestalt psychological notion, after which the term was coined. Closure, as I have shown, does not require traditional panels or gutters, does not require a consistent artistic style, may involve narrative ambiguity, and may incorporate multiple interrelated storyworlds or breaking of the fourth wall. Additionally, direct depictions of events, the presence of a story, and highly specific interpretations of narrative time beings depicted are not necessary for readers to enact closure. Since we have ruled all these things out, I will now detail my own views on what I take closure to involve.

\section{Closure as Coherence}

I aim to offer an account of closure that utilizes the strengths of McCloud's original conception, while incorporating the formalist critiques of Cohn's view and the flexibility and idiosyncrasy of Groensteen's notion of arthrology. Closure, then, should be understood as the non-sensorial cognition of a depicted coherent storyworld presented by the various images in a comics text. This understanding of closure maintains McCloud's focus on the active role of the reader and her imagination. ${ }^{100}$ Through their relationships to and engagement with sequences of images in comics readers enact

\footnotetext{
${ }^{99}$ McCloud, Understanding Comics, 96-97.

${ }^{100}$ McCloud, 65. "[Comics are] a medium where the audience is a willing and conscious collaborator and closure is the agent of change, time, and motion."
} 
closure. The inert works of comics do not contain closure, but only offer occasions for its enactment by active readers.

Additionally, this focus on the active understanding of coherence allows for the point (contra McCloud) that closure doesn't occur in the gutters of comics, even though gutters typically occasion closure. ${ }^{101}$ Closure is enacted through the relationships that readers have with the comics they read. Again, it is active readers who infer and imagine about the content of the sequences of images that make closure possible, not only the comics themselves. Visual or textual absences within the work (such as, gutters) do not typically communicate meaning directly, but they allow for and necessitate closure on the part of the reader-closure being the recognition of a coherent storyworld being communicated by the comic.

The recognition of "coherence" within a comics work suggests that the visual and linguistic information explicitly provided by the comic about the (potentially fictional) depicted storyworld is, at least, non-contradictory. ${ }^{102}$ However, non-contradiction is not enough of a constraint to suggest that any sequence of images will provide the opportunity for readers to enact closure. For example, a sequence of images may offer depictions of non-contradictory content that is simply unrelated. Unrelated depictions, while non-contradictory, are not necessarily sites of coherence. However, narrative coherence is not necessary in order for readers to enact closure, because while coherence

${ }^{101}$ Cohn, "The Limits of Time and Transitions," 136. "The gutter does not provide any meaning - the content of the panels and their union does."

${ }^{102}$ McCloud, Understanding Comics, 72. It may be the case that temporally impossible events may be depicted within comics (e.g., a strip featuring a time loop, in which cause and effect are bidirectional), but fully contradictory panels (e.g., panels that necessarily depict mutually exclusive storyworlds) may result in what McCloud calls "non-sequitur" panel transitions and may break down the visual narrative grammar of the sequence. 
requires more than only non-contradiction, it does not require the causal or temporal elements or relations necessary for a sequence to qualify as having a fully narrative structure. Again, juxtaposed images depicting two moments from a single event do not qualify as fully narrative.

Goldie's discussion of coherence in regard to narratives is useful here. As I mentioned above, Goldie argues that coherence is one of the characteristic features of narratives. While I am claiming that the recognition of coherence is integral for closure within comics, I potentially differ from Goldie in maintaining that coherence in this context is not sufficient for the presence of narrative. I am unaware of Goldie directly addressing whether coherence is sufficient for the presence of a fully narrative structure, but he does maintain that narratives must consist of representations of multiple events in a particular way. He writes, "like a causal account, a narrative cannot be concerned with just a single simple event; it must be about one thing happening after another, and the notion of coherence is concerned with how these things happening one after another hold together in some way." ${ }^{\prime 103}$ A level of coherence is necessary for the presence of narrative, but not sufficient, in my view.

The perception of a coherent storyworld in the medium of comics will likely include an element of temporality and/or causality. However, sequences of images that allow for closure need not communicate temporal duration within the world that is depicted. For example, panel-to-panel shifts may represent only perspectival changes in the spatial dimensions of the storyworld, rather than shifts in its temporal dimension. So

${ }^{103}$ Peter Goldie, The Mess Inside, 14. 
for a sequence of images to depict narrative content, ${ }^{104}$ on this view, temporal duration must be depicted in the sequence such that multiple events are understood to occur. Again, a sequence of images need not contain the depiction of multiple events, nor even a clear temporal element in the storyworld depicted, in order for closure to obtain. In the first two panels of the sequence below (Figure 6), no narrative time is depicted as passing. Rather, the first two panels represent only a shift in perspective.
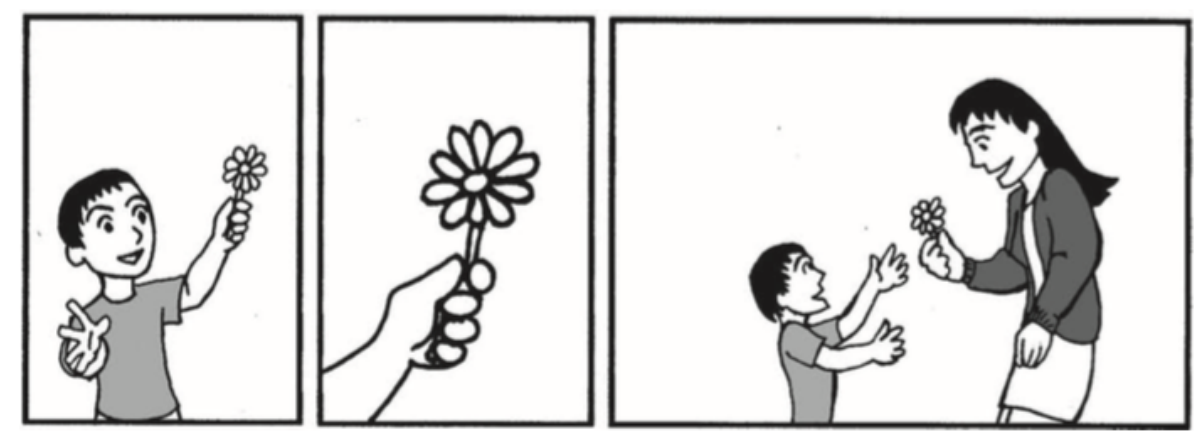

Figure 6: Cohn, "The Limits of Time and Transitions," 138.

Comics scholars John Bateman and Janina Wildfeuer adopt Segmented Discourse Representation Theory to explore the role of coherence relations in visual media, including comics. They write that discourse coherence relations in comics "mediate between the evidence that can be gleaned from an artifact under investigation [such as, a sequence of images] and the knowledge of an interpreter trying to understand that artifact. ${ }^{" 105}$ Coherence is, therefore, realized in the interaction between the reader and the work. In comics, the perception of coherence in sequences of images often will rely on both iconic (that is, visually mimetic) and conventional visual cues. As Bateman and Windfeuer note, the reader's prior knowledge and experiences will often influence her expectations, interpretation, and understanding of the content of the comic.

\footnotetext{
104 “Narrative," here, is used in Goldie's sense.

105 John Bateman and Janina Wildfeuer, "Defining Units of Analysis for the Systematic Analysis of Comics: A Discourse-based Approach," Studies in Comics 5, no. 2 (2014): 378.
} 
While sequential images may not adhere strictly to the syntactic conventions of written languages:

the fact that the discourse stratum is formally more free in no way entails that meanings are subject to whim or impressionistic interpretations. In order to function, it is necessary to be able to construct a coherent discourse structure employing the available rules for discourse coherence. ${ }^{106}$

This notion of coherence requires more than only non-contradiction, but does not require a fully narrative structure in Goldie's sense.

Understanding closure as implying recognition of coherence is continuous with psychological models of reader comprehension of textual works, as well. A coherent understanding of a work involves active reader engagement. The reader comprehends a work appropriately to the extent that she is able to mentally "construct a coherent situation model"107 with regard to the situation represented by the work. Comprehending a work (textual works, in this case) does not only involve a reader possessing a mental representation of the content of the work itself, rather the work should be thought of as "a set of processing instructions on how to construct a mental representation of the described situation." ${ }^{108}$ In other words, reader comprehension involves the understanding of a work as describing and depicting a coherent situation. In relation to sequential images this understanding arises via the non-sensorial cognitive processes that enable closure, by which readers imagine and infer a coherent storyworld.

\footnotetext{
${ }^{106}$ Bateman and Wildfeuer, "Defining Units of Analysis," 383.

${ }^{107}$ Rolf Zwaan and Gabriel Radavansky, "Situation Models in Language Comprehension and Memory," Psychological Bulletin 123, no. 2 (1998): 164.

108 Zwaan and Radavansky, 162.
} 
Local and Global Coherence via Closure: Imagination and Inference

The cognitive processes that enable closure include both imagination, as McCloud notes, and inference, as Cohn points out. The distinction between the roles of imagination and inference with regard to the process of closure can be described as a distinction between coherence in terms of time and space in the storyworld, and coherence in broader narrative terms. That is, when reading a comic, readers typically imagine spatiotemporal shifts from panel to panel and potentially between full-page frames (that is, the totality of all the panels on a full printed page of a comic attended to as one unit), ${ }^{109}$ while readers infer higher-order propositional narrative information from panel to panel or globally across an entire comics text. ${ }^{110}$

For example, a reader may imagine that the character "Bob" lifted a gun-from panel A where the gun is at his side, to panel B where he holds the gun out-but the reader infers that Bob is the killer when the reader sees that he is holding the gun (provided this information is not provided explicitly by the textual elements of the comic). This type of understanding of a work as coherent constitutes the process of closure when reading a comic.

This distinction between fine-grained imaginative cognition and more broad conceptual and narrative inferential cognition in comics is continuous with work on the comprehension of text-only works, such as short stories and novels. Text-only works,

\footnotetext{
${ }^{109}$ Eisner, Comics and Sequential Art, 65.

${ }^{110}$ Colin McGinn, Mindsight: Image, Dream, Meaning (Cambridge: Harvard University Press, 2006). It is worth quickly noting that this distinction resembles the distinction Colin McGinn makes between mental imagery (what I am calling "imagining") and cognitive imagination (what I am discussing in terms of "inference").
} 
similarly, require the reader to attempt to find coherence on both the local and global scales of the text. Graesser, Millis, et al. discuss this distinction, writing:

The explicit statements in a text need to be connected conceptually if the text is to be regarded as coherent. Local coherence is achieved if the reader can connect the incoming statement to information in the previous sentence or WM [working memory]. Global coherence is achieved if the incoming statement can be connected to a text macrostructure or to information much earlier in the text that no longer resides in WM. Readers normally attempt to achieve coherence at both the local and global levels. ${ }^{111}$

The achievement of coherence in a comic (as in a text-only work) will rely on the reader's working memory, the content of the work (both textual and visual), and her "background knowledge, modified by context and [her] goals." ${ }^{.12}$ Enacting closure in comics (and, thereby, achieving and understanding of the world of the narrative as coherent) requires the reader to perceive (in this case, to see) elements of the storyworld, rather than only to read about them. Thus, the imagining required in order for readers to adequately engage with comics is necessitated and constrained by the use of mimetic representational artwork.

The typical formal aspects of comics, involving sequential images rather than only textual signifiers, requires readers to engage in a uniquely imaginative way in comprehending the work as locally coherent. Readers see representations of the storyworld of the comic, but imagine both motion and time in response to the visual information delivered through the visual art presented in the work. Comics critic and theorist Douglas Wolk writes that "cartooning is, inescapably, a metaphor for the

${ }^{111}$ A.C. Graesser, K.K. Millis, et al., "Discourse Comprehension," Annual Review of Psychology 48 (1997): 178.

${ }^{112}$ Derek Matravers, Fiction and Narrative (New York: Oxford University Press, 2014): 63. 
subjectivity of perception." ${ }^{\text {113 }}$ Comics' approximations of subjective visual perception (and the resulting actual perception) dictate that readers enact closure across and within panels in visual terms, rather than in only conceptual and propositional terms, as readers might do when engaging with a text-only work.

The cartooning typically used in sequences in comics conveys meaning through mimetic representations, but also through more conventional non-textual symbols such as motion lines, speech balloons, and visual representations of sounds. These conventional symbols convey events within the storyworld of the comic, which add to the readers' understanding of aspects of narrative occurrences that may be difficult to depict. For example, there is empirical evidence that motion lines, specifically, "are conventionalized signs understood and expected by comic readers to appear in the depiction of motions and events [and the use of motion lines does] not simply originate in basic perceptual processes of the streaks left behind in the visual system." ${ }^{.14}$ So not only can readers acclimate themselves to these conventional means of event representation, but readers come to expect them when attempting to understand a comic.

This offers insight into the perceptual experiences of readers and can enlighten us as to the type of cognitive engagement involved in reading comics. Readers do not engage with comics only as visual approximations of potential perceptual experiences, as comics also utilize conventional representations of conceptual information. This is true not only with regard to the use of textual language, but also in terms of certain elements

\footnotetext{
${ }^{113}$ Wolk, Reading Comics: How Graphic Novels Work and What They Mean, 21. ${ }^{114}$ Neil Cohn and Stephen Maher, "The Notion of the Motion: The Neurocognition of Motion Lines in Visual Narratives," Brain Research 1601 (2015): 81.
} 
within the visual artwork used (e.g., motion lines, fight clouds, polymorphic panels, etc.). ${ }^{115}$

The use of these conventions typically distinguishes comics from children's picture books, another type of work featuring both text and pictures. While both mediums combine textual and visual artistic elements, the way those elements combine in order to convey meaning offer distinct reader experiences. Children's picture books typically do not employ panels and often contain one large picture per page. While some comics include pages that contain only one image (for instance, splash pages), this is a design tool that is only occasionally used for narrative emphasis or aesthetic weight. In other words, splash pages are non-standard in comics, but a single visual image accompanying the text on the page of a children's book is standard. Thus, one feature that typically distinguishes the reader experience of comics from that of children's picture books is the use of fine-grained imaginative closure (or local coherence) to communicate the movements of characters and other narrative events on a single page of the work.

It's important to note that the distinction between children's picture books and comics is not definitively structural or functional, but is socio-cultural. The fact that both mediums utilize text and image in order to communicate narrative information undercuts any solely functional distinction. As Cohn writes:

illustrated children's books are not comics quite simply because their definition finds no adherence with the non-structural conception of comics. Both genres occupy realms of cultural categorization, be it of readership, publishing, or content, though they share common elements of structure - text, images, and narrative. $^{116}$

${ }^{115}$ Cohn, "The Limits of Time \& Transitions," 132.

${ }^{116}$ Cohn, "Un-Defining 'Comics,"” 9. 
So while there are typical formal differences between children's picture books and comics, these differences are of degree and not necessarily of kind, and the distinctions between the two (structural and categorical) are dependent upon cultural expectations about the nature of the readers' engagement with the work. Again, "comics" is not a formal or linguistic category, but a category of cultural artifacts.

Joe Sutliff Sanders thoroughly examines the cultural underpinnings of the distinction between comics and illustrated children's literature in his article, "Chaperoning Words: Meaning-Making in Comics and Picture Books." ${ }^{" 117}$ Sanders cites the cultural history of comics in the early twentieth century U.S. and claims that the categorical distinction between comics and picture books is rooted in the emergence of a relatively unsupervised youth culture. Comics, typically featuring more and smaller works of art on each page, usually are intended to be read by an individual. Children's picture books, on the other hand, presume a "chaperone" to aid in understanding the meaning of the text. Sanders writes:

In determining who chaperones the words, a reliable and fertile difference emerges between comics and picture books: in general, if the book anticipates a solitary reader who chaperones the words as they go about their work of fixing the meaning of the images, that book is a comic; if the book instead anticipates a reader who chaperones the words as they are communicated to a listening reader, that book is a picture book. ${ }^{118}$

The larger images in picture books also highlight this presumption that two people will simultaneously engage with the work, as larger images are easier to show to another person while reading out loud. Comics, on the other hand, are "extremely awkward to

${ }^{117}$ Joe Sutliff Sanders, "Chaperoning Words: Meaning-making in Comics and Picture Books," Children's Literature 41 (2013): 57-90.

${ }^{118}$ Sanders, 61. 
read out loud."119 Again, this distinction is grounded in cultural reading norms, but has implications on the typical formal features of each type of work and, therefore, implications for reader experiences.

I have, to this point, argued that closure in comics should be understood as the reader's imaginative and inferential understanding of a locally and globally coherent storyworld. Imagination allows us to understand local spatio-temporal shifts in the storyworld, while inference allows us to comprehend more conceptual narrative elements globally across larger sections of a comic. I have also discussed how comics are distinct from text-only works and children's picture books. Going forward, the relation of comics to film is worth exploring, as the comparison between the two mediums is often made.

\section{Distinction Between Comics and Film}

Comics and film are both visual mediums, so the comparison is understandable. However, some have endorsed the misconception that comics do not offer experiences that are sufficiently divergent from those associated with film to necessitate independent philosophical investigation. Philosopher Roy T. Cook explains this misconception, writing, "so the worry goes, comics, properly understood, are (or are equivalent to) a subspecies of film, and as a result all of the tools and techniques we need to evaluate and understand them can be found in film theory and the philosophy of film." ${ }^{\prime 120}$ The formal

\footnotetext{
119 Sanders, 74.

${ }^{120}$ Roy T. Cook, "Why Comics are Not Films: Metacomics and Medium-Specific Conventions," in The Art of Comics: A Philosophical Approach, eds. Aaron Meskin and Roy T. Cook (Malden, MA: WileyBlackwell): 165.
} 
and cultural-historical similarities between comics and film can encourage this misconception. $^{121}$

This view is not wholly unreasonable, as comics and film do share many significant formal qualities. Both mediums "tend strongly to be narrative" 122 and involve sequences of typically mimetic images that communicate narratively relevant events occurring within the presented storyworld. Films also necessitate viewer engagement that is somewhat like closure in comics. Henry John Pratt writes that, "both [comics and film] employ largely mimetic, visual narration; both are prototypically gappy; and both control the percipient's attention to a similar degree and with similar techniques." ${ }^{\prime 23}$ However, films require viewers to actively fill different "gaps" than do comics.

Specifically, it is the juxtaposition of images in comics that most clearly distinguishes closure in comics from the type of "gappiness" in film. Films replace one image with another in order to create the illusion of motion and the regulation of time, while comics typically position multiple images simultaneously on a single surface. This implies that the perceptual experience of the reader of comics will differ from that of the viewer of film in that the comics reader's visual field will include multiple images depicting various points in narrative time from various perspectives in the storyworld of the comic. Cook argues for the aesthetically unique formal features of comics, writing:

The fact that scenes in a film are projected - that is, their order and duration are controlled solely by the filmmakers (and perhaps the projectionist) - while panels in a comic can be perused in whatever order we decide - paging back and forth,

${ }^{121}$ Henry John Pratt, "Making Comics into Film," in The Art of Comics: A Philosophical Approach, eds. Aaron Meskin and Roy T. Cook (Malden, MA: WileyBlackwell): 149. ${ }^{122}$ Pratt, 155.

${ }^{123}$ Pratt, 155. 
comparing earlier and later images - suggests that it is not just the order of the relevant units [that is, panels], but the nature of this ordering, that is different. ${ }^{124}$

As explained above, on a fine-grained imaginative level, readers of comics must enact closure in order to account for their understanding of motion within the narratives. This is also true of the pacing of the narrative and, to some extent, the narrative time that is understood to have elapsed across the gaps between panels, as well as within panels. ${ }^{125}$

The visual-artistic conventions used to suggest constraints on interpretations of narrative motion and time also distinguish the experiences of engaging with comics from engagement with films. Films provide the perceptual illusion of motion and time, creating a much more constrained role for the viewer. While the conventional visual-artistic elements typically used in comics may be incorporated into film, this does not suggest that the viewing of a film can reproduce the experience of engaging with a comic. As Cook writes, "It is not that we cannot have speech or word balloons as ingredients of films - quite the contrary [...] The point is that, as used within film, they are already a non-standard feature, typically functioning as an explicit reference to comics." ${ }^{126}$ Obviously, filmmakers may visually reference the typical formal features of comics in their films, but the nature of film as a medium negates the possibility of creating the same experiences for audiences as the comics medium provides.

So, the perceptual illusions of motion, time, and pacing are present in the viewing of films, but are absent in the reading of comics. Enacting closure within comics requires

\footnotetext{
${ }^{124}$ Cook, "Why Comics Are Not Films," 173-174.

${ }^{125}$ This is not a completely unconstrained interpretive process enacted by readers, obviously. The content of the relevant panels will place constraints on reasonable interpretations of the duration of narrative time from across or within panels, as was mentioned in the discussion of polymorphic panels.

${ }^{126}$ Pratt, "Making Comics into Film," 182.
} 
readers to actively imagine and infer those elements of the storyworld, using the explicit content contained within the comic. While illusions of motion and time are absent in comics, juxtapositions of static images are (typically) absent in films. Readers of comics, then, must draw differentially on the "information sources" at their disposal, including text, background knowledge, context, and goals, in order to experience a coherent storyworld presented by a comic. ${ }^{127}$ This results in a perceptually and cognitively distinct experience with regard to the viewing of films, compared to the reading of comics.

\section{Two Examples of Depicted Coherence}

In order to exemplify the conception of closure as cognitive processes resulting in the understanding of the comic as depicting a globally and locally coherent storyworld, I will briefly discuss two examples from mainstream U.S. comics. The first example comes from one of the most influential comic books in American history, Action Comics \#1. This issue was released in June of 1938 and features the first appearance of Superman, a character often considered to be the paradigmatic superhero.

In the first Superman story, entitled "Superman: Champion of the Oppressed," the protagonist is depicted engaging in several different heroic acts including saving someone wrongly accused of murder, thwarting a corrupted politician, and rescuing reporter Lois Lane from the clutches of a gangster. Preceding Lois's kidnapping by the gangster, she insults the gangster when he insists that she dance with him. Clark Kent (Superman's secret identity) pretends to be a coward, so as not to arouse suspicion about his heroic true identity.

${ }^{127}$ Matravers, Fiction and Narrative, 63. 
In the panels below (Figure 7), the gangster threatens Lois Lane after she refuses to dance with him. Following his threat, Lois slaps the gangster across the face, prompting Clark to say, "Lois - don't!," while thinking, "Good for you, Lois!", ${ }^{128}$ This juxtaposition of panels exemplifies many of the issues that have been discussed in this chapter. Each panel includes a clear black border and the gutter is rendered in the typical way, as a blank white space on the page. Additionally, these panels include examples of speech balloons, thought bubbles, and caption boxes. Motion and impact lines also appear in this sequence, highlighting the use of non-mimetic conventional representations of events in the storyworld.
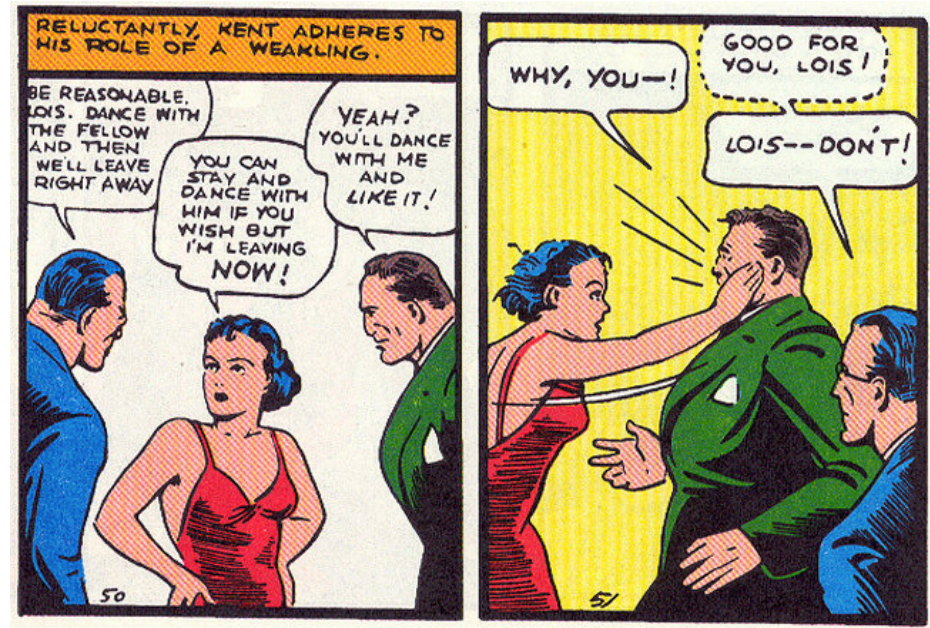

Figure 7: Jerry Siegel and Joe Schuster, The Superman Chronicles Volume One (New York: DC Comics, 2006).

Due to the similarity of their depictions, the reader can identify that in both panels the same three characters appear, but at different temporal points in the narrative. ${ }^{129}$ The similarities include the dimensions of their faces, as well as the colors and styles of their clothing. So, when enacting closure with regard to this juxtaposition of images, readers

\footnotetext{
${ }^{128}$ Jerry Siegel and Joe Schuster, The Superman Chronicles Volume One (New York: DC Comics, 2006): 10.

${ }^{129}$ Here, I use the term "narrative" because this juxtaposition is part of a larger work. If these panels existed as a work on their own, they may or may not represent a "fully narrative structure" in the sense previously discussed.
} 
imagine the spatio-temporal shifts from the first to the second panel. The speed and ferocity with which Lois's arm moves to slap the gangster (taking into account the motion and impact lines) must be included in the reader's imagining of the depicted events.

The imagining of these panel-to-panel shifts in the spatio-temporal dimensions of the storyworld constitutes reader recognition of local coherence in this comic. While readers are likely to infer Lois's attitude in this situation (i.e., frustration with Clark's cowardice), ${ }^{130}$ this conceptual aspect of the narrative is not absent from the depictions in the same way as are Lois's speed and motion, because Lois's mental states constitute propositional information about the storyworld at the global level. Therefore, a reader inferring Lois's attitude toward Clark, which seems to span the entirety of the narrative (due to her ignorance of his true identity), represents the inferred recognition of global coherence.

The second example comes from a much later instantiation of the Superman character. The popular creative team of writer Jeph Loeb and artist Tim Sale offered their take on the Superman mythology with 1998's Superman For All Seasons. This limited series presents a story set early in the career of Superman and features characters that have become staples of Superman's storyworld, including Lois Lane. In the sequence below (Figure 8), we again see Lois Lane defending herself from a threatening criminal. This sequence is, no doubt, inspired by the above sequence (Figure 7), which appeared sixty years prior. The sequence below also displays many of the formal features of U.S. comics that I have discussed so far. The panels are clearly distinguished by black borders

${ }^{130}$ This inference is confirmed in panel fifty-four. 
and the gutters appear in standard fashion as blank white spaces between the juxtaposed artwork.

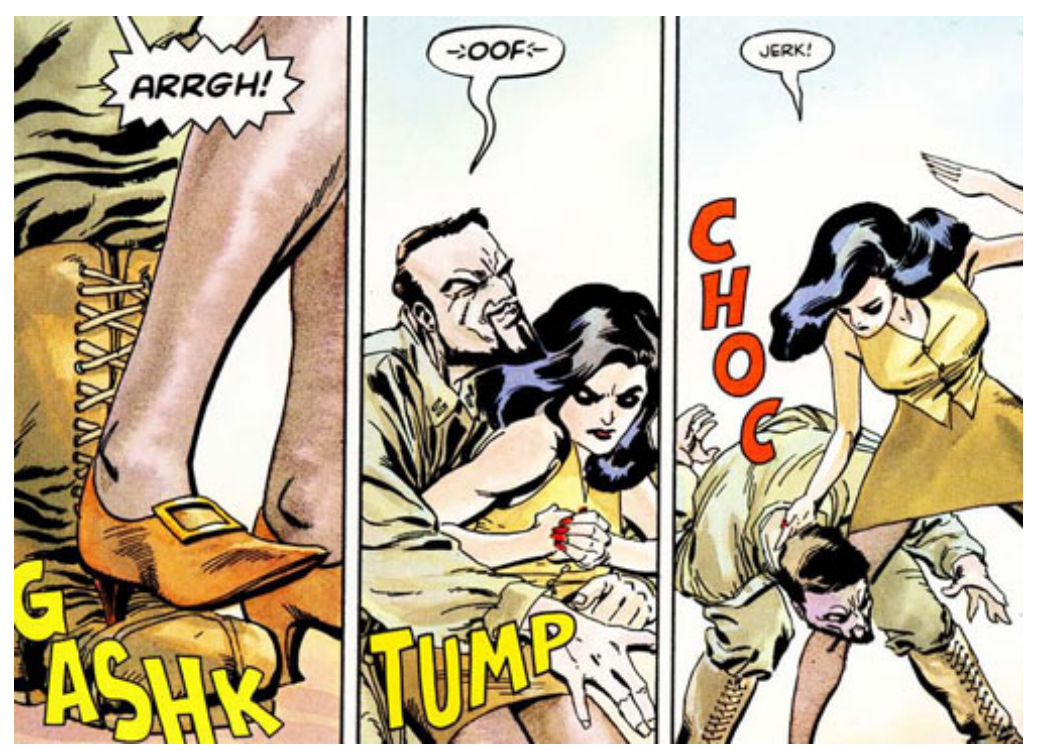

Figure 8: Jeph Loeb and Tim Sale, Superman for All Seasons (New York: DC Comics, 1999).

This sequence does include a few features that are not present in the prior sequence, including free-floating onomatopoetic words within the panels. These are intended to relay aural information about the depicted situation to the reader visually. These exemplify "visualized sound." 131 Also, the perspectives shift more in this sequence than in the previous example. The first panel presents a close up of Lois's foot as she stomps on the foot of the criminal and the second and third panels present a wider view of the bodily positions of both Lois and the criminal. Despite the shifts in perspective, readers are able to enact closure and recognize both of these sequences, respectively, as depicting a coherent storyworld.

While the approaches to story telling, narrative pacing, and artistic styles has undoubtedly evolved over the past century in U.S. comics, the basic formal features that allow for the enactment of closure by readers have remained very similar since their early

${ }^{131}$ Duncan and Smith, The Power of Comics, 144. 
days. As Groensteen writes, "the story [in a comic] is possibly full of holes, but it projects me into a world that is portrayed as consistent, and it is the continuity attributed to the fictional world that allows me to effortlessly fill in the gaps of the narration." ${ }^{, 132}$ Formally typical comics contain gaps in their narratives that readers must actively fill in, such that the storyworld of the comic is understood as being locally and globally coherent.

\section{Conclusion}

As I have argued, there is likely no element or set of elements that are necessary and sufficient for the instantiation of a comic. Comics will typically be pieces of media that utilize multiple static images and text in the service of evoking a particular aesthetic response or communicating a narrative ${ }^{133}$ Rather, comics should be thought of as a socio-cultural artistic category that typically, but not necessarily, manifests certain features (such as, panels, cartooning, text, speech balloons, etc.). Comics, as a cultural category, should not be conflated with the visual languages that comics employ. ${ }^{134}$

However, the lack of necessary and sufficient features for comics does not present an obstacle to examining the typical cognitive (e.g., imaginative and inferential) and perceptual (e.g., viewing mimetic and other visual representations) experiences offered to readers of comics or the uniqueness of those experiences when compared to engagement with other forms of artistic media, including novels, static visual artworks, picture books, and films. The process of closure, which is necessary for readers to properly comprehend most comics, involves both imagination and inference for readers to recognize a coherent

\footnotetext{
132 Groensteen, The System of Comics, 11.

${ }^{133}$ This basic requirement mirrors McCloud's definition of comics to a large extent.

${ }^{134}$ Cohn, "Un-Defining 'Comics,'” 9-11.
} 
storyworld presented by the comic. On the local level, this amounts to the imagining of shifts in time and space from panel to panel (and page to page) within the storyworld of the comic. And on the global level, readers must infer conceptual and propositional information that is useful in understanding the meaning of the work.

The fact that comics readers enact closure also functions to influence the types of aesthetic affordances and opportunities for unique emotional engagements available to those who engage with comics. Readers' emotional experiences in response to comics are shaped by the roles of character depiction, artistic style and design features, in addition to the concept of closure that I have detailed above. These perceptual, cognitive, and emotional experiences ultimately influence the notions of selfhood implied by many comics stories, and the cultural place that comics have typically occupied. It is to these issues that I now turn. 


\section{II: AESTHETIC POSSIBILITIES OF EMOTIONS IN COMICS}

\section{Emotions as Processes}

Now that we have dealt in detail with the role that closure plays in our understandings of the meanings of comics, I will turn to the significant role that emotional engagement plays in our experiences when we read comics. Because I will be offering an understanding of the emotional responses afforded by the typical formal features and narrative content of comics in the American cultural context, it is necessary to outline the understanding of emotions with which I am operating.

It should be noted that I will not be arguing that the following account of the nature of emotions is necessarily generalizable to all (or even most) cases of emotional responses. Rather, I will argue for a particular philosophical model of emotions that is most relevant to understanding readers' typical engagement with comics. Whether or not this model of emotions functions in other contexts is irrelevant. I will maintain that the account of emotions that I affirm can best account for our emotional engagements with comics.

Within the literature on philosophy of emotions, a recurring division is found between various "perception" theories of emotions and various "judgment" theories of emotions. Perception theories of emotions tend to focus on the bodily and felt aspects of emotions, while judgment theories tend to emphasize the conceptual and cognitive 
elements of emotional responses. I will argue that both of these standard models are found wanting when exploring emotions in relation to comics, and that an understanding of emotions as multi-stage processes that involve both physiological and conceptual elements can more sufficiently accommodate the emotional engagements typically afforded by comics. I will take these theoretical approaches to emotions in turn.

Perception theories of emotions tend to frame emotions as physiological in nature and as "bodily changes of various sorts" 135 that are available to the emoting individual to categorize upon reflection. William James famously put forth a perception theory of the nature of emotions, ${ }^{136}$ usually called the "James-Lange" theory. ${ }^{137}$ On this view, emotions just are perceptions of physiological changes in an individual and those physiological changes may precede the person's understanding or conceptualizing of the emotional state constituted by those changes.

James argues "bodily changes follow directly the perception of the exciting fact, and that our feelings of the same changes as they occur is the emotion $[\ldots]$ we feel sorry because we cry, angry because we strike, afraid because we tremble." ${ }^{138}$ Many current influential thinkers defend various perception theories of emotions, including philosopher Jesse Prinz ${ }^{139}$ and neuroscientist Antonio Damasio. ${ }^{140}$ Perception theories of emotions are

${ }^{135}$ Jenefer Robinson, Deeper Than Reason: Emotion and its Role in Literature, Music, and Art, (New York: Oxford University Press, 2005), 36.

${ }^{136}$ William James, "What is an Emotion?" Mind, 9 (1884): 189-190. See also, Carl Lange and William James, The Emotions, ed. Knight Dunlap (Baltimore: Williams \& Wilkins, 1922): 33-90.

${ }^{137}$ Ronald de Sousa, "Emotion," The Stanford Encyclopedia of Philosophy (Spring 2014 Edition), edited by Edward N. Zalta, accessed October 28, 2017. https://plato.stanford.edu/archives/spr2014/entries/emotion/.

${ }^{138}$ James, "What is an Emotion?" 188-205.

${ }^{139}$ Jesse Prinz, Gut Reactions: A Perceptual Theory of Emotion (New York: Oxford University Press, 2004). 
sometimes labeled as "non-cognitive,"141 because they privilege the physiological, sensorial, affective, and non-conceptual aspects of emotional responses. That is, perception theories typically conceive of emotions as being primarily defined in terms of felt bodily changes.

Prinz, who put forth an influential and nuanced account of emotions that relies heavily on non-cognitive elements, argues that:

Emotions are not in fact cognitive, most of the time. They are not generated by acts of cognition, and they are not conceptual. We have conceptualized versions of our emotions, and we can use these in cognitive acts, but in ordinary cases emotions are not cognitive at all. ${ }^{142}$

For Prinz and others who promote non-cognitivist or perceptual accounts of the nature of emotions, certain conscious conceptual elements may accompany emotional states, but they are not considered constituent elements of those emotions.

A conception of emotions that privileges non-conceptual aspects of emotional responses, however, faces difficulty when attempting to understand our typical engagements with comics. Comics typically deal with conceptual content that is communicated in multiple ways. As per the discussion above, distinguishing comics and visual language, conceptual content is delivered in comics through both their pictorial and textual elements. Typically the images in comics are representational (often mimetic) and the textual elements conceptually reference the storyworld of the comic.

The concepts referenced by the text and artworks of comics clearly influence the types of emotional engagements that readers may experience. Because of this, typical

${ }^{140}$ Antonio Damasio, "Fundamental Feelings," Nature, 413 (2001): 781.

${ }^{141}$ Remy Debes, "Neither Here nor There: The Cognitive Nature of Emotion," Philosophical Studies 146, no. 1 (2009): 6.

${ }^{142}$ Prinz, Gut Reactions, 50. 
critiques of perception theories seem to hold in relation to our emotional experiences of comics. For example, in order for a reader to be relieved that Batman has defeated the Joker's plot to poison the Gotham City reservoir in Batman: The Man Who Laughs, ${ }^{143}$ the reader must think that certain propositions hold with regard to the storyworld of the comic. For instance, she must think (believe, judge, evaluate, etc.) that the goals of the Batman character are admirable, those of the Joker character threatening, and she must understand the narrative as depicting the successful realization of Batman's goals. Her relief cannot be explained only in terms of her perception of a physiological response. In fact, it is possible that her physiological response would not be intense enough to reach the threshold of her awareness, but it still could present a subtle emotional tone to her experience that she does not perceive.

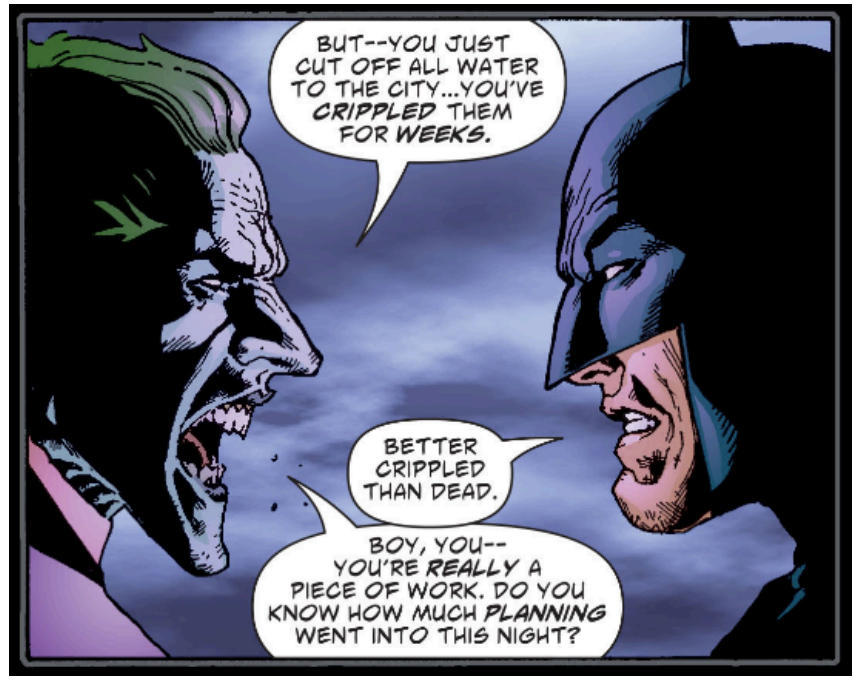

Figure 9: Ed Brubaker and Doug Mahnke, Batman: The Man Who Laughs (New York: DC Comics, 2008), 67.

Perception theories of emotions will have difficulty reckoning with emotional states that are conditioned on judgments or conceptual content, but that involve physiological shifts that the emoting individual does not perceive. In this example, we

${ }^{143}$ Ed Brubaker and Doug Mahnke, Batman: The Man Who Laughs (New York: DC Comics, 2008). 
must invoke the readers' conceptualizations of and judgments about the narrative content of the Batman comic in order to fully understand her emotional experience. She is relieved that Batman has saved the day. We must also incorporate her understanding of the narrative as a series of representations rather than as something happening in her immediate environment. However, as we will see, overemphasis on only the conceptual elements of emotional responses can also be problematic.

An alternative family of philosophical accounts of the nature of emotions focuses on the role of the propositional and conceptual content associated with emotions. This type of view emphasizes the evaluative judgments that the emoting individual makes regarding the intentional objects associated with emotions (e.g., what situation the emotional response is directed toward or is about). These views often are called, aptly, “judgment" theories of emotions. ${ }^{144}$ Thinkers going as far back as the Stoics have advocated judgment theories of emotions. ${ }^{145}$

Judgment theories have an intuitive appeal. It is clear that emotions often involve judgments about their intentional object(s). For example, doesn't my fear of George entail that I make an evaluation of George, which implies certain beliefs, and judge him to be a threat? Many judgment theorists would say yes. So, according to this type of view, emotions must be directed toward, and feature an evaluative judgment regarding, their relevant intentional object(s). We cannot merely feel fear, anger, or sadness in regard to a particular situation without having made a judgment about that situation, these theorists claim.

\footnotetext{
${ }^{144}$ Robinson, Deeper Than Reason, 8-14.

${ }^{145}$ Martha Nussbaum, "Emotions as Judgments of Value and Importance," In Series in Affective Science. Thinking About Feeling: Contemporary Philosophers on Emotions, ed. R. C. Solomon (New York, NY, US: Oxford University Press, 2004): 185.
} 
Judgment theorists sometimes frame the cognitive or conceptual elements of emotions as both necessary and sufficient for the instantiation of emotions, even occasionally going so far as to fully identify emotions with those judgments. ${ }^{146}$ Philosopher Martha Nussbaum claims that in order to experience an emotion, an individual must hold certain evaluative beliefs. She offers the example of her own emotional response to the death of her mother. Her tremendous emotional response to that event in her life, she argues, required the adherence to the truth of the proposition that her mother had died.

Nussbaum writes, "severing emotion from belief, [...] severs emotion from what is not only a necessary condition of itself, but a part of its very identity." ${ }^{147}$ So, for judgment theorists like Nussbaum, emotions are inherently conceptual phenomena. In order to have a truly emotional response to a situation, one must hold certain beliefs about and embody a certain perspective toward that situation. ${ }^{148}$ Experiencing an emotion is, at least in part, necessarily to make an evaluative judgment about the intentional object(s) to which the emotion is a response.

There are problems with judgment theories of emotions, as well. A general and well-noted critique of theories that privilege the conceptual content of judgments associated with emotions is that judgments alone are "neither necessary nor sufficient for

\footnotetext{
${ }^{146}$ Nussbaum, 196.

${ }^{147}$ Nussbaum, 189.

${ }^{148}$ Nussbaum, 191. Nussbaum particularly associates the cognitive elements with the distinction between the identities of various emotions, writing, "the cognitive elements are an essential part of the emotion's identity and of what differentiates it from other emotions."
} 
an emotion." ${ }^{149}$ To address the question that I introduced above: No, I do not have to judge George to be a threat in order to feel a fear response to him. I may consciously judge George to be a perfectly amiable person and still experience a feeling of fear when in his presence that I find inexplicable. Alternatively, I may feel perfectly calm around George even though I know that he is a very violent man.

With regard to comics specifically, judgment theories of emotion will have trouble offering an account of emotional responses to the less straightforwardly conceptual or representative elements of comics art. As I will explore in detail below, many of the visual artistic and design elements in comics potentially can shape the affective and emotional responses of readers in ways that don't involve the explicit judgments. For example, the color palette used in a comic may influence the emotional tone of the narrative and, therefore, affect the range of emotions typically experienced by readers.

If a comic features dark, sketchy, and high-contrast visual art, which leads a reader to have an unsettled feeling, it is likely to contribute to the emotional response of the reader without fully determining her conceptualization of the content of the storyworld or her understanding of the narrative events of the comic. In other words, contents of the reader's visual field may condition a physiological response that is part of her emotional experience but that does not obviously rely upon her evaluative judgments about any straightforwardly conceptual elements of the comic with which she is engaging.

149 Julien A. Deonna and Fabrice Teroni, The Emotions: A Philosophical Introduction (New York: Routledge, 2012), 54. 
While both judgment and perception theories of the nature of emotions have strengths, the common distinction between models that emphasize cognition and those that de-emphasize it may be an overly generalized and potentially misguided one. Philosopher Remy Debes argues that the distinction between the cognitive and noncognitive understandings of the nature of emotions is founded on a distinction that makes little difference. He writes, "So-called cognitivists and anti-cognitivists disagree about the nature of emotion only with respect to degrees cognitivity-and maybe not even that." ${ }^{\text {150 }}$ Debes reframes cognitivity as a "cluster" 151 concept, which can be used to articulate various levels and types of information processing achieved by the emoting individual. Cognitivity of emotions, on this view, involves various elements that can be used to situate emotional responses and experiences on "a kind of spectrum"152 of degrees to which the emotion in question is cognitive in nature. Debes' innovative approach to the relation of cognitivity to emotions tracks nicely with the understanding of the nature of emotions that most illuminates the emotional affordances typically offered by comics.

Philosopher Jenefer Robinson, drawing heavily on empirical psychological work showcasing an appraisal model of emotions, ${ }^{153}$ argues that emotions should be understood as multi-stage processes, rather than as states. Robinson's argument that emotions should be understood as sequences of events is convincing and highly illuminative when considering emotional engagements with comics. To reiterate, I am not arguing that Robinson's model of emotions is universally applicable or that it serves to undermine other accounts of emotions in response to other stimuli. Rather, this model of

${ }^{150}$ Debes, "Neither Here Nor There: The Cognitive Nature of Emotion," 5.

${ }^{151}$ Debes, 12 .

152 Debes, 15.

${ }^{153}$ Robinson, Deeper Than Reason, 57-99. 
emotions as processes is best served to elucidate the multifaceted ways in which comics allow us to emotionally engage (e.g., mimetic artwork, non-representational artistic features, textual elements, narrative structure).

The understanding of emotions that will most clearly illuminate our emotional engagement with comics is the view that emotions involve both physiological responses and conscious conceptual elements. This model of emotions as processes allows for Debes' insight that emotions manifest varying relationships to and levels of cognitivity. Robinson details the typical unfolding of the process of an emotion, writing:

An affective appraisal draws attention to something in the environment significant to me or mine and gets my body ready for appropriate action. Then immediately cognitive evaluation kicks in, checks the affective appraisal to see if it is appropriate, modifies autonomic activity, and monitors behavior. More complex cases of emotion in human beings might involve affective responses not to a perception but to a thought or belief, and the cognitive monitoring may be correspondingly sophisticated, but at the core of emotion will always be physiological responses caused by an automatic affective appraisal and followed by cognitive monitoring. ${ }^{154}$

So, while those elements of emotions that are usually the focus of perception theories (such as, physiological events and automatic affective appraisals) are key to emotional responses, in characteristic cases of emotions, those elements are then regulated by those elements of emotions that are usually the focus of judgment theories (such as, conceptual or propositional information processing and monitoring).

Two aspects of Robinson's account make it especially relevant to understanding the emotional affordances of comics. First, while emotional responses function in service of "evaluating the environment in terms of how it affects the organism," 155 they need not be in response to things that are external to the organism. In other words, affective

\footnotetext{
${ }^{154}$ Robinson, 59.

${ }^{155}$ Robinson, 19.
} 
appraisals (the initial stage of emotional processes) may originate in response to concepts or propositions within the mind of the emoter, rather than as a result of external objects or events in their immediate surroundings. This is not to say that these concepts or propositions function as the intentional objects of the emotions in question, but rather that complex conceptualizations can provoke less obviously cognitive affective appraisals, which are subsequently cognitively monitored.

Robinson offers the example of being afraid about the performance of one's stock portfolio, a situation that requires complex conceptual engagement in order to adequately understand it. Examples such as this show that one can have a "rough and ready" affective physiological response to a situation, the relevance of which only becomes clear after "lengthy cognitive evaluations." ${ }^{156}$ In relation to comics, this entails that readers may have physiological affective appraisal responses to their understandings of the complex storyworld presented by a comic. This allows for the insight of perception theories that emotions involve physiological changes, while allowing that complex and abstracted understandings of situations may condition such changes.

The second point of Robinson's account that makes it ideal for exploring emotions in relation to comics is that, on this account, emotions do not necessarily involve any particular beliefs about the fictionality of the intentional object(s) of the emotional response. This point is key because, whether a comic is taken to be fictional or non-fictional, the reader of comics is responding to a depicted storyworld represented within the comic. The reader of comics, then, may respond emotionally to both the perceptual experiences afforded by and the conceptual content presented in a comic,

${ }^{156}$ Robinson, 62. 
regardless of whether or not she takes the events represented to be actual historical events or impossible fictional ones. Robinson writes:

Pre-cognitive affective appraisals do not discriminate between the real and imagined scenarios: I respond emotionally to whatever seems to have a bearing on my interests and on those to whom I am close (my family, my group, my fellow humans). It does not matter to my emotion systems (fear, anger, sadness, etc.) whether I am responding to the real, the merely imagined, the possible or the impossible. $^{157}$

For example, if the reader sees the depiction of a beloved character lying in a casket at a funeral, she may experience sadness in response. In this example, the reader of comics has an affective appraisal response (that is, an automatic response to "those things in the $[\ldots]$ environment that matter" $)^{158}$ to her conceptualization of a funeral that is prompted by a visual representation, rather than in response to the sight or experience of an actual funeral.

The subsequent cognitive monitoring (that is, information processes by which we “control and modify our responses") ${ }^{159}$ of her own physiological response, which constitutes the affective appraisal of the perceptual experience and resulting conceptualization, allows her to distinguish appropriate from inappropriate actions in response to the depiction of the dead character in the comic. ${ }^{160}$ For example, simply furrowing her brow or even crying may be appropriate, while purchasing flowers as a condolence will likely be an inappropriate response. ${ }^{161}$

\footnotetext{
${ }^{157}$ Robinson, 145.

158 Robinson, 42.

${ }^{159}$ Robinson, 89.

${ }^{160}$ Robinson, 153.

${ }^{161}$ This aspect of conceiving of emotions as processes is relevant to the philosophical problem known as the "paradox of fiction."
} 
Note that the response of purchasing flowers to mourn a character who is depicted as deceased will typically be inappropriate (and therefore, not undertaken) whether the comic is understood to be fictional or non-fictional. The relevant distinction when thinking about our behavior in response to narrative works, as philosopher Derek Matravers rightly argues, is not between fictional and non-fictional narratives, but between "confrontations and representations." 162 In other words, it does not matter whether or not a reader believes that the events depicted in the comic ever actually happened, or even whether those events could have actually happened. Either way, the reader is engaging with and responding to representations of events, characters, and settings that are not happening currently in her immediate surroundings and her behavior will normally reflect that. ${ }^{163}$

Another example is that of perceiving a character who is depicted as threatening. McCloud's example of the axe murder, if presented in the right context, might elicit a fearful response in the reader. However, while an anxious facial expression or nervous chuckle may be appropriate (or even inevitable), cognitive monitoring will typically keep the reader from trying to duck the swinging axe or save the screaming victim. This distinction between appropriate and inappropriate action responses is the same even if the axe murder is taken to be depicting an historical event.

\footnotetext{
162 Matravers, Fiction and Narrative, 53.

${ }^{163}$ Matravers, 50. "In representation relations our mental states are not caused by perceptions of the objects of those states, and do not result in actions toward objects in our egocentric space (although, of course, they can still cause actions)."
} 

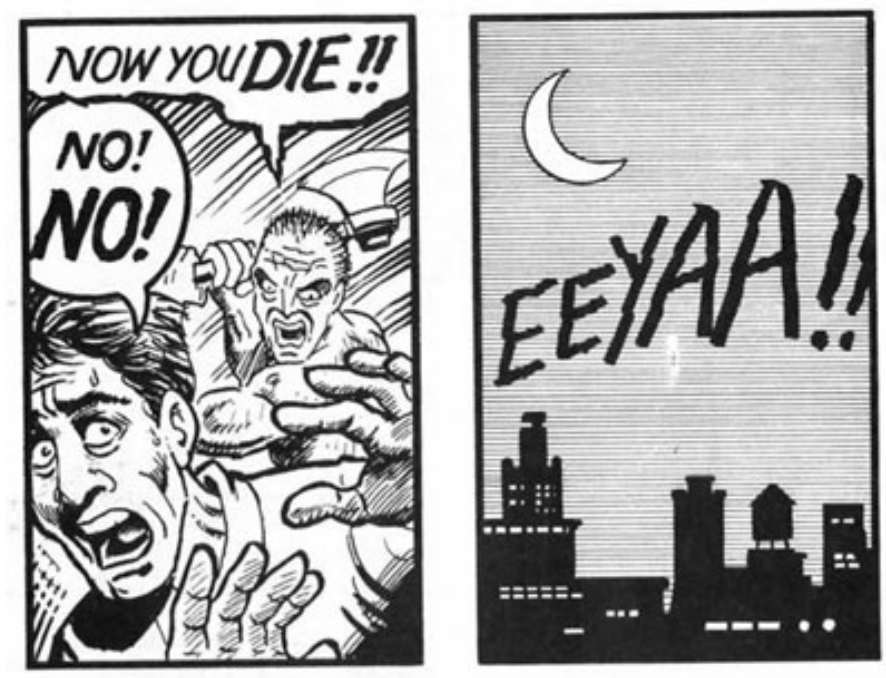

Figure 10: McCloud, Understanding Comics, 66.

As I discussed in the previous chapter, when the reader of comics enacts closure, she will typically understand the storyworld of the comic to be a coherent one. Therefore, readers may respond emotionally to either depicted content or to implied content that is available only through the enactment of closure. The images of the comic provide guidance to the reader in imagining and inferring the narrative content that is not directly represented in the work. The content represented in a particular panels' artwork is constantly perceptually available to structure and enrich the readers' understanding (and imagining of) narrative events. ${ }^{164}$

When engaging with a piece of visual art, the physical depiction becomes "no longer a concrete object that provides me with perception: it serves as matter for the [mental] image." ${ }^{\prime 165}$ But because comics are constituted by multiple sequential artworks, readers will oscillate between direct perceptual engagement with the comic and enacting closure (inferring and imagining narrative information that is not explicitly depicted or

\footnotetext{
${ }^{164}$ Jean-Paul Sartre, The Imaginary: A Phenomenological Psychology of the Imagination (New York: Routledge, 2004): 23.

${ }^{165}$ Sartre, The Imaginary, 21.
} 
described). All of the narrative content, explicit or implicit, potentially can shape and be shaped by the reader's emotional experiences.

Many of our emotional responses to comics will be conditioned by what we imagine and infer as readers. However, as I will discuss in detail near the end of this chapter, our emotional responses to certain elements of comics also may influence our imaginative engagement with the work and thereby influence our enactment of closure (that is, our emotions may influence how we understand the meaning of the comic). For example, we may imagine a particular character's movement as being a particular speed because of the comic's sketchy art style or muted color palette.

While I will focus mostly on those opportunities for emotional engagement afforded by the directly depicted content of comics, content that must be either imagined or inferred may also be part of the reader's understanding of the story world presented by the comic and, therefore, may condition emotional responses via affective appraisals. Before continuing to the ways in which the typical formal conventions of comics shape readers' emotions, I will discuss the role that time plays in emotional engagements with narratives, broadly.

Narrative, Time, and Emotional Responses

The interaction of an individual's emotions and the narratives with which they engage (regardless of the medium in which the narratives are delivered) are time sensitive. That is, reader emotions and a particular narrative will interrelate in different ways over time. First, the emotional state and tendencies of a potential reader "has an influence [on the individual's relation to a narrative] before one even engages with a 
story." ${ }^{, 166}$ Before a reader interacts with a narrative, her current emotions and her goals regarding possible future emotional experiences may influence her choice of which narrative to engage.

For example, if she is currently sad and wishes to be happy, she may choose a narrative that she anticipates will have that effect on her. ${ }^{167}$ Further, she may choose a narrative medium that she anticipates will align with her emotional goals. If an individual wants to feel fear, she may choose to watch a film rather than to read a novel if she anticipates more of a fear response from visual-audio engagement than from text (perhaps because of her previous experiences with the various mediums). In short, she may anticipate the type of emotional response that her engagement with a particular narrative genre or medium, or combination of the two, will result in for her.

During the reader's interaction with a narrative, the content of the story (such as, characters, setting, perspective, plot, etc.) may provide occasions for emotional responses of various types, including empathy, sympathy, and emotional contagion (that is, when readers "catch" the emotions displayed by a character). ${ }^{168}$ Emotional responses like these, which rely on the reader taking a perspective within the story, are sometimes called "narrative feelings," while "aesthetic feelings" ${ }^{169}$ result from taking a perspective

\footnotetext{
${ }^{166}$ Raymond A. Mar, et al., "Emotion and Narrative Fiction: Interactive Influences Before, During, and After Reading," Cognition and Emotion 25, no.5 (2011): 818. ${ }^{167}$ Mar, et al., 820. Anticipated emotional responses and their relation to the choice of narratives are more complex than this example accounts for, as meta-emotions (i.e., evaluations and feelings about one's current or anticipated emotional state) can also affect the choice of which, if any, narrative to engage.

${ }^{168}$ Amy Coplan, "Catching Characters' Emotions: Emotional Contagion Responses to Narrative Fiction Film," Film Studies 8, no.1 (2006): 28.

${ }^{169}$ Miall, David S., and Don Kuiken, "A Feeling for Fiction: Becoming What We Behold," Poetics 30, no. 4 (2002): 223.
} 
"outside" the text. ${ }^{170}$ Both may be operative over the course of engaging with the narrative. The former may arise via emotional contagion, while the latter may arise from what is called "criterial prefocusing." I will discuss both emotional contagion and criterial prefocusing in greater detail below.

Finally, readers' emotional responses often continue after the readers have finished engaging with the narrative. After reading a narrative, "the deep simulation of experience that accompanies our engagement"171 with stories can continue to influence our emotional dispositions and responses into the future. For example, readers may seek to emulate the emotional attributes of those characters that a narrative frames in a positive light. Alternatively, they may consciously avoid emotional tendencies that are reflective of antagonists within stories. I will argue in the next chapter that the formal features of comics suggest, independently of their narrative content, a certain understanding of the nature of the characters depicted within them. This reading of the nature of depicted characters may carry over into readers' understandings of themselves, as well.

While shifts in readers' emotional dispositions or tendencies may be short lived, they may also result in long-term changes to readers' personalities. ${ }^{172}$ Some empirical work suggests that emotional responses to narrative artworks are a mediating factor in the ability of narratives to influence readers' perceptions of themselves, "given that human psyche appears to respond to the artistic form through subtle shifts in the vision of

\footnotetext{
${ }^{170}$ Mar, et al., "Emotion and Narrative Fiction," 822.

${ }^{171}$ Mar, et al., 829.

${ }^{172}$ Mar, et al., 829.
} 
itself." $" 173$ In other words, "emotion [is] central to the experience of change in the ways in which [readers view] themselves [after engagement with a narrative], that is to say in their personality." ${ }^{\prime 74}$ If, as I will argue in the third chapter, the formal elements of comics implicitly offer readers' particular conceptions (and potentially re-understandings) of their own selfhood, the emotional significance of a work will influence the impact of the self-conception that comics readers take away from their reading experience.

Within this chapter, I will mainly focus on the potential for emotional engagement during and after reader interaction with comics, although the choice to read a comic will undoubtedly be influenced by a reader's emotional disposition regarding the cultural place that comics hold and, potentially, the reader's previous experiences with them. For example, if I have been bored reading comics before, then I may be less likely to choose to read a comic in the future.

Before going forward, it is important to note that I am not concerned with determining the range of possible emotional experiences that are afforded to readers of comics. I do not take my arguments to depend on the question of whether emotions are primarily socially and culturally constructed, and are therefore potentially unconstrained in their diversity, or whether there is some biologically constrained set of base emotional responses that are only calibrated by their cultural context. ${ }^{175}$ Whichever of these views of the nature of the potential variety of emotions (or any view in between) is the right one should not have bearing on the claim that comics offer readers uniquely constituted

${ }^{173}$ Maja Djikic, Keith Oatley, Sara Zoeterman, and Jordan B. Peterson, "On Being Moved by Art: How Reading Fiction Transforms the Self." Creativity Research Journal 21, no. 1 (2009): 28.

${ }^{174}$ Mar, et al., "Emotion and Narrative Fiction," 829.

${ }^{175}$ Paul Ekman, "Basic Emotions," in Handbook of Cognition and Emotion, eds. Tim Dalgleish and Mick J. Power (New York: The Guilford Press, 1999): 45-60. 
opportunities for emotional engagement. The nature of the affordances can be unique to the comics medium whether the range of emotions and emotional responses is very narrow and determinate, or potentially endlessly variable.

In other words, I will not claim that comics offer opportunities for unique emotional experiences. ${ }^{176}$ To give a simplistic example, sadness can be experienced when engaging with a comic, but this does not entail that sadness is a response that is unique to engaging with comics. Films and novels may also induce sadness in readers and viewers. Rather, the ways in which comics may invoke sadness (and other emotional states) in the reader are unique. I will claim that the types of opportunities for emotional engagement in relation to comics are unique to the medium, not the emotions themselves.

Because narratives can be rendered in any number of artistic mediums, including novels, plays, and film, the emotionally salient aspects of a narrative will be communicated in ways that are dependent on and determined by the medium's formal properties, material instantiation, and perceptual mode(s) of presentation. For example, films may engage our emotions through their musical scores, sound design, the pace of editing, composition of shots, and the timing of actors' line delivery. As Jan Baetens and Hugo Frey write, "The choice of the medium induces a set of possibilities as well as impossibilities, of obstacles as well as chances, that are not found in other media." ${ }^{, 177}$ The medium of comics presents a unique set of possibilities for readers to engage emotionally.

\footnotetext{
${ }^{176}$ Neither will I deny this.

${ }^{177}$ Jan Baetens and Hugo Frey, The Graphic Novel: An Introduction (New York: Cambridge University Press, 2015): 162.
} 
In the previous sections, I have detailed potential philosophical approaches to understanding the nature of emotions. I have argued that while both perception and judgment theories of emotions have their strengths, the relationship of comics and emotions is best understood using Robinson's model of emotions as processes involving both affective physiological and cognitive conceptual elements. We have also seen that comics can influence (and be influenced by) readers' emotional states before, during, and after their engagement with a particular comic.

Moving forward, I will illuminate several formal aspects of comics that can offer medium-unique emotional affordances for readers. I will concentrate on three ways in which comics uniquely afford opportunities for emotional engagement: the depiction of characters, artistic style and design elements, and the need for readers to enact closure. I do not take these three aspects to be exhaustive of the ways in which comics can offer opportunities for readers to emotionally engage. However, each of these elements (as well as the amalgamation of the elements) contributes to the totality of reader experiences in relation to comics and the opportunities for emotional responses afforded by them.

\section{Emotional Salience in Comics through Character Depiction}

Graphic narratives, being linguistically bimodal, ${ }^{178}$ have the capacity to depict characters' emotional states, behaviors, and psychological dispositions rather than only describing them. The capacity for comics to depict rather than only linguistically reference the emotionally salient features of the storyworld provides opportunities for reader engagement via the reader's visual system that are unavailable in textual works, such as novels. For example, depictions of faces can allow readers to infer the mental

${ }^{178}$ Cohn, “Un-defining ‘Comics,” 3. 
states of characters, empathize with the depicted characters, and arrive at emotionally salient judgments regarding the characters' psychological and behavioral tendencies.

The visual language used in most American comics depicts characters, events, and contexts iconically (that is, mimetically through resemblance) rather than only symbolically (that is, with arbitrary or conventional representations). ${ }^{179}$ Iconic representation within comics offers a low comprehension cost for the reader, ${ }^{180}$ allowing her to see and easily garner information about aspects of the storyworld of the comic and not only imagine them. ${ }^{181}$ "In graphic narratives, verbal description of emotional states can be replaced by drawings of bodily postures and facial expressions that readily communicate feelings to readers." ${ }^{\text {182 }}$ So, certain emotionally salient features of a narrative can be shown to the reader as well as being described or suggested by the textual elements of the comic.

Textual descriptions and images of situations convey information in distinct ways. As philosopher John Kulvicki argues, "images present their contents to us in a way that mimics the way in which we perceptually acquire information more generally." ${ }^{183}$ That is, typically we acquire information about our environment visually and images convey information in a way that capitalizes on this capacity. Therefore, images wield an epistemological weight when it comes to the information they represent visually (in this

${ }^{179}$ Cohn, The Visual Language of Comics, 19.

${ }^{180}$ Brian Boyd, "On the Origin of Comics: New York Double-Take," Evolutionary Review 1 (2010): 105.

${ }^{181}$ McCloud, Understanding Comics, 67. This is not to suggest a passive reader experience. Imagination, expectation, and reader inference play a large part in the comprehension of comic narratives, as I explained in detail in the previous chapter. ${ }^{182}$ Suzanne Keen, "Fast Tracks to Narrative Empathy: Anthropomorphism and Dehumanization in Graphic Narratives," SubStance 40, no. 1 (2011): 146.

${ }^{183}$ John Kulvicki, "Knowing with Images: Medium and Message," Philosophy of Science 77 , no. 2 (2010): 310. 
case, information about the storyworld of the comic). This epistemological weight is due to the fact that images can convey "a great many pieces of abstract information," whereas textual "descriptions $[\ldots]$ are very selective in the pieces of abstract information that they provide." ${ }^{\prime 184}$ A picture actually may be worth a thousand words. The ways in which we engage with images, particularly mimetic ones, parallels the way in which we usually gain information about our immediate environment visually.

For example, if a character is textually described as "distraught," a reader may imagine that character wearing any range of appropriate facial expressions or bodily postures. Whereas, if a character is represented as in the image below (Figure 11), the reader may ascribe any number of appropriate emotionally salient labels to the facial expression depicted. ${ }^{185}$ While a literary text offers readers textual descriptions of the storyworld, comics offer both textual conceptual information ${ }^{186}$ and also mimic the visual experiences one would have if one were actually confronting those aspects of the storyworld. This allows the reader to imagine and infer information about the storyworld as a result of her direct perceptual experiences.

\footnotetext{
${ }^{184}$ Kulvicki, "Knowing with Images: Medium and Message," 298.

${ }^{185}$ The reader need not explicitly verbalize or label (internally or otherwise) what she takes the nature of the emotional state depicted to be or her judgments regarding that state. Her experience of those depicted emotional states, being visual, has the potential to be automatic via emotional contagion.

${ }^{186}$ Cohn, "The Limits of Time and Transitions," 133. Comics offer conceptual information in at least two ways: textual content and polymorphic panels that represent conceptually, rather than mimetically, their depicted content.
} 

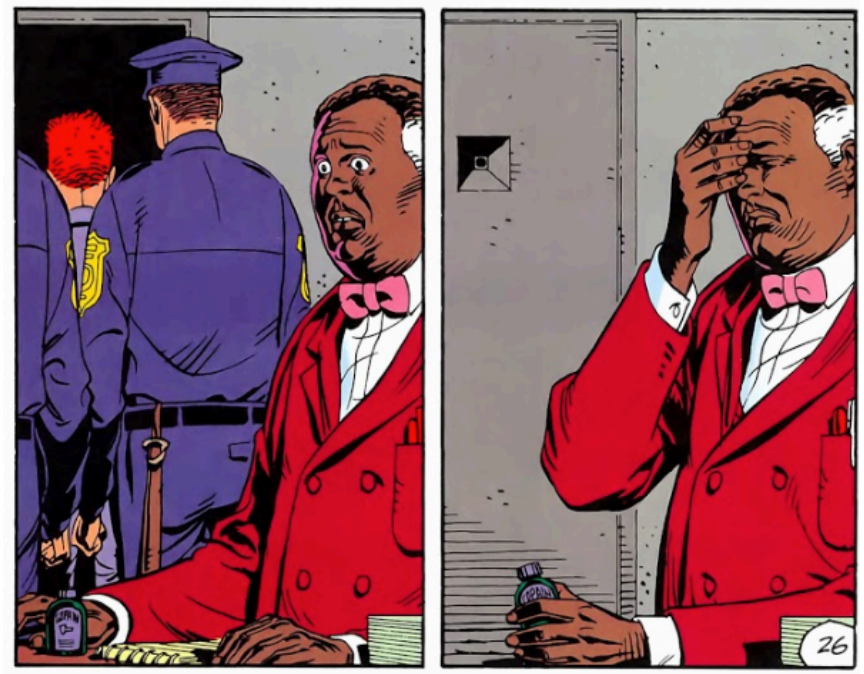

Figure 11: Alan Moore and Dave Gibbons, Watchmen (New York: DC Comics, 1986-1987): Chapter VI, 26.

Iconic representations of characters allow opportunities for readers to have emotional contagion responses to depictions of emotionally salient facial cues. Emotional contagion occurs when one experiences (to some extent) the emotional state that she observes in another as a result of how she perceives the other's emotional state. The process of emotional contagion "requires direct sensory engagement and involves automatic processes." ${ }^{187}$ It occurs when observers of others' emotions unconsciously mimic the emotionally salient aspects of the individual(s) they are observing, those mimicked facial and bodily responses influence the observer's self-perceived emotional state, and the observer "[ends] up 'catching' the emotions of those they observe"188 as a result of observing them.

In other words, a reader may have an automatic physiological affective appraisal response to depictions of particular aspects of a storyworld, including characters' facial expressions. When reading a comic, you see a distraught character and your physiology may mimic the character's distress without any conscious volition on your part. As you

${ }^{187}$ Coplan, "Catching Characters' Emotions," 26.

${ }^{188}$ Coplan, "Catching Characters' Emotions," 28. 
immediately begin to cognitively monitor your own physiological response, you realize that you also are feeling distress and can come to understand that feeling in terms of the narrative events in which the character is situated. You need not be aware of the stages of this process; rather you simply feel distress as you view and read about the distressed character.

Typical cases of emotional contagion involve directional contagion from one living person to another when they are interacting in real-time. However, visual representations of agents provide sufficient conditions for emotional contagion as well. ${ }^{189}$ There is some empirical evidence that a viewer's "disposition for emotion contagion,", is a highly influential factor in how that viewer experiences her engagement with representational visual artwork. ${ }^{191}$ That is, those who are more likely to experience emotional contagion experience visual art "as more moving, more interesting, and [they provide] more extreme valence [that is, evaluative] ratings. ${ }^{.192}$ While viewers of films are afforded this type of opportunity for emotional engagement, ${ }^{193}$ comics also include the representational visual element required for emotional contagion while requiring a distinct type of cognitive engagement (that is, closure) that films do not. ${ }^{194}$ Readers of

${ }^{189}$ Freeberg, David and Vittorio Gallese, "Motion, Emotion and Empathy in Esthetic Experience," Trends in Cognitive Science 11, no. 5 (2007): 201.

${ }^{190}$ Gerger Gernot, Matthew Pelowski, and Helmut Leder, "Empathy, Einfühlung, and Aesthetic Experience: The Effect of Emotion Contagion on Appreciation of Representational and Abstract Art using fEMG and SCR," Cognitive Processing 19, no. 2 (2018): 149.

${ }^{191}$ Gernot et al., 163.

${ }^{192}$ Gernot et al., 159.

${ }^{193}$ Coplan, "Catching Characters' Emotions," 28.

${ }^{194}$ Coplan, "Catching Characters' Emotions," 34. In fact, Coplan's article exploring emotional contagion in relation to film uses sequences of still images taken from films to illustrate her points. The use of these images itself suggests the possibility of emotional contagion with regard to sequences of static images. 
comics must imagine and infer elements of the storyworld that viewers of film more passively observe.

However, philosopher Noël Carroll argues that the effect of emotional contagion is often overestimated when attempting to understand readers' emotional responses to narratives. As evidence, he cites the epistemological asymmetry that often exists between characters within a storyworld and those engaging with the representation of that storyworld. ${ }^{195}$ He writes, "in some cases we know more than the characters; we tremble for them as they plunge ahead ignoring clear and present danger. On the other hand, Sherlock Holmes always knows more than we do, so we never share his aplomb in the face of peril." ${ }^{\prime 196}$ This asymmetry between reader and character may undermine emotional contagion as an explanation in cases where the narrative is crafted to guide the reader toward an emotional response that diverges from that of the character being depicted or described.

Carroll puts forth an alternative explanation for cases that are generally understood as emotional contagion, or as he calls it, "infectious identification." 197 His alternative, which he calls "criterial prefocusing," emphasizes the ways in which the elements of narratives craft an expected emotional response from the perspective of a

${ }^{195}$ Noël Carroll, "On Some Affective Relations Between Audiences and the Characters in Popular Fictions," in Empathy: Philosophical and Psychological Perspectives, eds. Amy Coplan and Peter Goldie (New York: Oxford University Press, 2011): 179-80.

Although Carroll does acknowledge the potential role that mirror responses can play in contagious "affective states," he does not believe that these constitute "full scale emotional states" because they "do not necessarily involve appraisals, though they may afford data pertinent to forming an appraisal."

${ }^{196}$ Carroll, 168.

${ }^{197}$ Carroll, 171. 
reader, rather than relying on the emotions of the characters to be the sole or primary

influence over readers' emotional responses. Carroll offers the following example:

When the monster in the concluding scenes of Bride of Frankenstein is reviled by his reanimated betrothed, we feel sorry for him. Our emotion does not match his. We do not feel the pain of the unrequited lover. Indeed, I doubt that any viewers, no matter how desperate, harbor any desires for the frizzy-haired, electrified corpse, played by Elsa Lanchester. But we do respond to the monster's misery with sorrow. It is in this sense that we share his misery. We are not miserable for being lovelorn but we do pity the monster. ${ }^{198}$

Thus, in cases of criterial prefocusing, "the situation [in the storyworld] has already been prestructured for our attention" 199 by the author, who has chosen which elements of the narrative she will highlight or emphasize in order to attempt to evoke the emotional response she desires from the audience. In other words, Carroll holds that various narrative elements, beyond just the communication of characters' emotional states, shape the audience's attention in a way that makes certain features of the storyworld differently emotionally relevant than they would be from the perspective of any character within the narrative.

While I agree that criterial prefocusing may explain many of our emotional engagements with narratives, this does not rule out the potential effect of emotional contagion responses altogether. This is especially true given the understanding of emotions as processes involving involuntary affective appraisals and given the representational visual nature of comics, both of which make emotional contagion likely. Criterial prefocusing relies on a more judgment-focused understanding of emotions, which is only part of the story of emotional processes.

\footnotetext{
198 Carroll, 172.

${ }^{199}$ Noël Carroll, A Philosophy of Mass Art (Oxford: Clarendon Press, 1998): 262.
} 
The reader has a differing perspective on the situation than does Frankenstein's monster, and therefore, her judgments about the situation are distinct. However, as was discussed above, emotions are processes involving both affective appraisals and cognitive monitoring, and comics offer readers visual experiences that may carry emotional weight even before they are conceptualized and before judgments are made about the storyworld being represented. Therefore, both criterial prefocusing and emotional contagion may be operative on readers of comics.

Comics also provide readers with opportunities for both empathic and sympathetic responses to characters, due to the inclusion of character depiction. While emotional contagion is automatic and initiated by subpersonal processes, sympathy and empathy both involve more conscious and conceptual engagement with a work. Philosopher Amy Coplan writes, "sympathy involves caring about another individual— feeling for another. It does not as such involve sharing the other's experience."200 Sympathy, then, involves aspects of conscious thought in a way that emotional contagion does not.

This designation of sympathy is fairly common and therefore, sympathy can be understood in this context as a reader's emotional response that is distinct from and conditioned upon the depicted or described emotional state of a particular character or characters. For example, seeing the character in Figure 11 looking distraught may cause the reader to feel sorry for him. The sympathetic reader does not feel distress that is the same as the character's depicted state or simulate within herself the first-personal emotional perspective of the character, but rather experiences her own distinct emotional

\footnotetext{
${ }^{200}$ Amy Coplan, "Empathic Engagement with Narrative Fictions," The Journal of
} Aesthetics and Art Criticism 62, no. 2 (2004): 145. 
perspective based on her conception of the depiction of the character's emotional state, as well as other relevant narrative factors.

Emotional affordances are also available to readers of comics in the form of empathic responses. "Empathy" is a somewhat contested term and there are many "competing conceptualizations" within the philosophical literature, which encompass "several loosely related processes or mental states." 201 Coplan defines empathy as "a complex imaginative process involving both cognition and emotion,"202 through which the individual's (in this context, the reader's) experiences are similar to the target's (character's) “emotional states, while [the reader is] simultaneously imaginatively experiencing his or her cognitive states." ${ }^{203}$ So sympathy may be experienced for those characters one feels for but doesn't necessarily identify with, while empathy involves imaginatively simulating for oneself the first-personal experiences of a character.

It is important to note that Coplan's understanding of empathy may or may not utilize the concept of "imagination" in the way I have used it in my account of the process of closure in the previous chapter. Again, I am using "imagination" to refer to a mental process involving internal representations of potentially sensory information to oneself. ${ }^{204}$ In my view, imagining necessarily has a phenomenological element to it. In the cases relevant to this work, the sensory information communicated will represent a storyworld by way of the visual art, textual elements, and design elements of any particular comic.

${ }^{201}$ Amy Coplan, "Understanding Empathy: Its Features and Effects," in Empathy: Philosophical and Psychological Perspectives, eds. Amy Coplan and Peter Goldie (New York: Oxford University Press, 2011): 4.

202 Coplan, "Empathic Engagement," 143.

203 Coplan, 144.

${ }^{204}$ Currie and Ravenscroft, Recreative Minds, 12. 
Coplan, discussing imagination, writes, "to say that empathy is 'imaginative' is to say that it involves the representation of a target's states that are activated by, but not directly accessible through, the observer's perception." ${ }^{205}$ Coplan is silent on the question of whether she takes imagination to necessarily involve a phenomenological element. I affirm a reading of her account of empathy on which imagination includes a phenomenological element. Such a reading of Coplan's notion of empathy should be compatible with my understanding of closure, and therefore with my account of emotional engagements afforded by comics.

Peter Goldie complicates the picture of empathy by distinguishing between what he calls "in-his-shoes" perspective-shifting and "empathetic" perspective-shifting. ${ }^{206}$ These both involve attempting to predict and understand the responses and feelings of another. The in-his-shoes approach involves imaginatively putting oneself into the position of another and the empathetic approach entails that one attempt to simulate elements of the other person's "characterization." Elements of characterization, in Goldie's view, include "not only traits of character and of personality, but also intellectual traits and abilities, such as open-mindedness and quick-wittedness, and emotional dispositions, such as being compassionate towards the homeless, or loving one's spouse. ${ }^{207}$ Goldie argues that the fully empathetic approach to perspective-shifting does not and cannot represent our typical attempts at understanding the positions of others because elements of characterization play a "covert, non-speaking part in the

\footnotetext{
${ }^{205}$ Coplan, "Understanding Empathy," 5-6.

${ }^{206}$ Peter Goldie, "Anti-Empathy," in Empathy: Philosophical and Psychological Perspectives, edited by Amy Coplan and Peter Goldie (New York: Oxford University Press, 2011): 303.

${ }^{207}$ Goldie, 308.
} 
deliberation" of perspective-shifting that cannot be replicated consciously by the individual attempting the empathetic approach. ${ }^{208}$

The requirements for a fully empathetic experience are simply too demanding. That is, I can attempt to feel, believe, respond, and know, in ways that are identical to a particular character, but I will be unlikely to succeed because elements of my own characterization will still be quietly shaping my perspective. While I affirm Goldie's position on the unlikeliness of what he calls the empathetic approach, both the in-hisshoes approach and the empathetic approach to perspective-shifting are compatible with the ways in which character depiction affords opportunities for emotional engagement to readers of comics.

Empathic and sympathetic emotional responses are afforded to readers of comics through what they see. And further, the types of visual representations used in a work can affect the likelihood of particular emotional responses in readers. McCloud argues that more abstracted depictions of characters (such as, cartoon style drawings) allow readers to more easily identify ${ }^{209}$ with characters so depicted, whereas more detailed and realistic depictions serve to create distance between the reader and character by representing them as part of "the world outside," ${ }^{210}$ or the environment of the reader. McCloud writes:

By de-emphasizing the appearance of the physical world in favor of the idea or form, the cartoon [drawing style] places itself in the world of concepts. Through traditional realism, the comics artist can portray the world without-- --and through

\footnotetext{
${ }^{208}$ Goldie, 316.

${ }^{209}$ Coplan, "Empathic Engagements with Narrative Fictions," 141. It seems that McCloud is using the term "identification" to mean roughly what Coplan means by "empathy." Coplan avoids the term "identification," claiming that its use in the literature on empathy tends to be "somewhat vague or ambiguous."

${ }^{210}$ McCloud, Understanding Comics, 40.
} 
the cartoon, the world within $[\ldots]$ viewer-identification is a specialty of cartooning. ${ }^{211}$

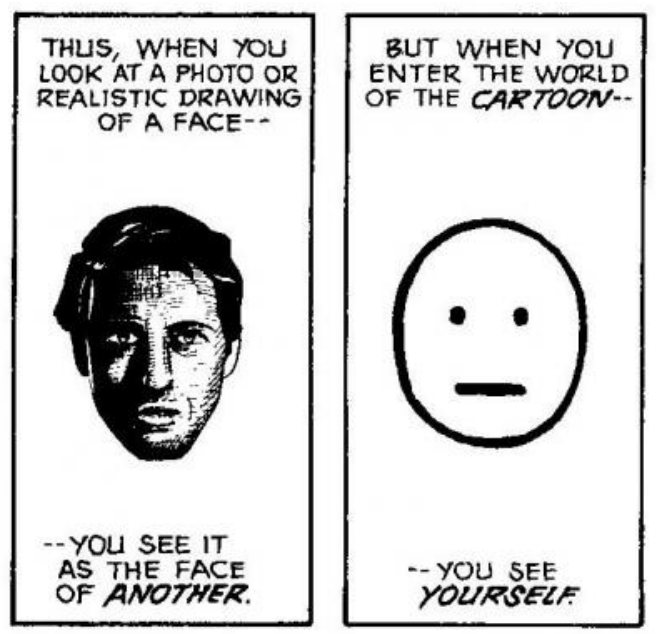

Figure 12: McCloud, Understanding Comics, 36.

Taking McCloud's point into consideration, artistic style may serve to vary the range and types of opportunities readers have for emotional engagement with particular characters in comics. Sympathy (that is, feeling for a character) may be more or less likely than empathy (that is, imaginatively simulating the emotional and cognitive states of a character) as a function of the artistic style employed in depictions of that character within a work. Specifically, sympathy in readers would be more likely than empathy in cases where the character is represented in a photo-realistic drawing style.

Empathy, according to McCloud, is more likely in cases where the characters are depicted in abstracted and cartoony styles, which allow for easier reader-identification. McCloud offers the panel below (Figure 13) as an example, in which the background and setting is depicted photo-realistically and the main character is depicted in a cartoony drawing style, allowing "readers to mask themselves in a character and safely enter a sensually stimulating world." ${ }^{212}$ Whether or not McCloud's analysis of the role of photo-

\footnotetext{
${ }^{211}$ McCloud, 41-42.

${ }^{212}$ McCloud, 43.
} 
realism is accurate is an empirical question, but he offers a plausible hypothesis of how readers' emotional responses may potentially vary as a function of degrees of realism with regard the depictions of characters, rather than only as a function of the conceptual content represented in those depictions.



Figure 13: McCloud, Understanding Comics, 43.

Another role that empathic responses may play in the emotional experiences of comics readers is the potential for readers to identify, not with a particular depicted character, but with the emotional perspective of the work as a whole. ${ }^{213}$ This potential function of empathic response resembles Carroll's criterial prefocusing in that the perspective taken is not that of a particular character and thus the broader features of narrative may affect the reader's responses.

However, when a reader experiences empathy with a work as a whole, she does not simply respond to expectations implicit in the work about the perspective of an audience member, rather she identifies with the perspective that the work takes toward the issues, events, or concepts with which the work engages. This empathic reader

${ }^{213}$ Elisabeth Camp, "Two Varieties of Literary Imagination: Metaphor, Fiction, and Thought Experiments," Midwest Studies in Philosophy 33, no.1 (2009): 122-24. 
response, which is a type of "aesthetic feeling," ${ }^{214}$ may potentially affect the perspective of readers regarding themselves and their understanding of the world around them.

As in all narratives, reader responses of both sympathy and empathy will be conditioned on "the life experience and even the emotional state of each reader,", ${ }^{215}$ but her emotions will interact with and be affected by the particular character of her visual perception in the case of comics. "Many narrative artists aim at moving readers' feelings, and graphic narration brings a tool kit of visual arts techniques to enhance the effort."216 These empathic, sympathetic, and emotional contagion responses, as well as those responses conditioned by criterial prefocusing, need not affect the reader only during her time with the narrative. As discussed above, emotional responses have the potential to shape one's behavior and perspective after one has finished engaging with the work. ${ }^{217}$

In the previous section, we have seen how emotional contagion, criterial prefocusing, empathy, and sympathy are all made possible for readers of comics through character depictions. However, depicting characters' emotional states does not involve only mimicking the actual visual experience of encountering characters with such emotions. Some empirical evidence suggests that certain cultural artistic conventions (beyond mimetic representation) are used in communicating emotional content to readers of comics. ${ }^{218}$ The examples of these cultural artistic conventions in the following section will highlight the overlap between the emotional impact of images of characters and the

${ }^{214}$ Miall and Kiuken, "A Feeling for Fiction," 223.

${ }^{215}$ Duncan and Smith, The Power of Comics: History, Form, and Culture, 153.

${ }^{216}$ Keen, "Fast Tracks to Narrative Empathy," 136.

${ }^{217}$ Keen, 152-153. Keen discusses the role of depicted emotionally salient aspects of graphic narratives and their potential for encouraging prosocial behavior and ethical social change. How to practically realize such potential is an open question.

${ }^{218}$ Xiaojuan Ma, Jodi Forlizzi, and Steven Dow, "Guidelines for Depicting Emotions in Storyboard Scenarios," 8th International Design and Emotion Conference, 2012. 
particular artistic style utilized. This leads us from emotions in response to depictions of characters to the emotional role of artistic style and design elements in comics more broadly.

Emotional Salience through Artistic Style and Design Elements

Artistic style and design elements can influence readers' emotional responses to and their understandings of the content and meanings of comics in several ways. One way that comics writers and artists depict emotional salience stylistically is through "hermeneutic images." ${ }^{219}$ Hermeneutic images do not "represent sounds or objects that exist in the world of the story; instead they comment on the story itself [and they] imply more than what is literally shown. ${ }^{, 220}$ Psychological hermeneutic images depict emotionally salient aspects of the characters' or authors' psychological perspectives toward elements of the storyworld by metaphorically (rather than iconically) depicting them.

For example, in her graphic novel Bitchy's College Daze, Roberta Gregory depicts her parents in a style that metaphorically represents their emotional states and character traits through artistic depiction. She depicts her mother as "so vacuous that her face consists of only a smile and big eyelashes" and her father with "a mouth full of long fangs and squiggly lines emanating from his body." ${ }^{221}$ These stylistic choices represent the emotional tone of Gregory's perspective on her parents and "the constant embarrassment she feels" ${ }^{\prime 222}$ regarding them. In doing so, her artistic style guides the

\footnotetext{
219 Duncan and Smith, The Power of Comics: History, Form, and Culture, 159.

${ }^{220}$ Duncan and Smith, The Power of Comics: History, Form, and Culture, 159.

${ }^{221}$ Duncan and Smith, 160.

${ }^{222}$ Duncan and Smith, 160.
} 
emotional responses, perspectives, and experiences of her readers. The reader is afforded the opportunity to adopt, at least during the reading of the comic, the author's (and protagonist's) emotional outlook on the situation.

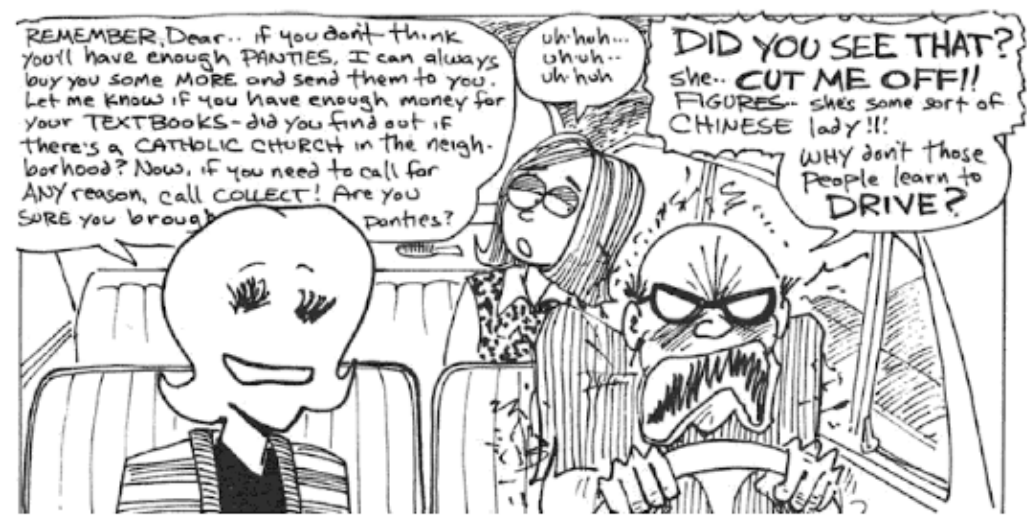

Figure 14: Roberta Gregory, Bitchy's College Daze (Seattle: Fantagraphics, 1995). reprinted in Duncan and Smith, The Power of Comics, 160.

Craig Thompson's graphic memoir entitled Blankets also contains examples of psychological hermeneutic images that serve to communicate the author's perspective and evoke similar emotional experiences in the reader. Thompson recounts an occasion when he attempted to renounce his love of drawing in the name of religious devotion. Through the teachings of the religious community in which he was raised, he came to see drawing as "escapism" and "the most secular and selfish of worldly pursuits!"223 In order to make a new spiritual pact with his god, Thompson burns all of his artwork. The fullpage panel that depicts him burning the drawings includes sketches flowing from his mouth as his eyes roll back in his head. Thompson offers this stylized hermeneutic image in order to visually communicate his emotional state at the time.

${ }^{223}$ Craig Thompson, Blankets (Montreal, Canada: Drawn and Quarterly, 2015): 58. 


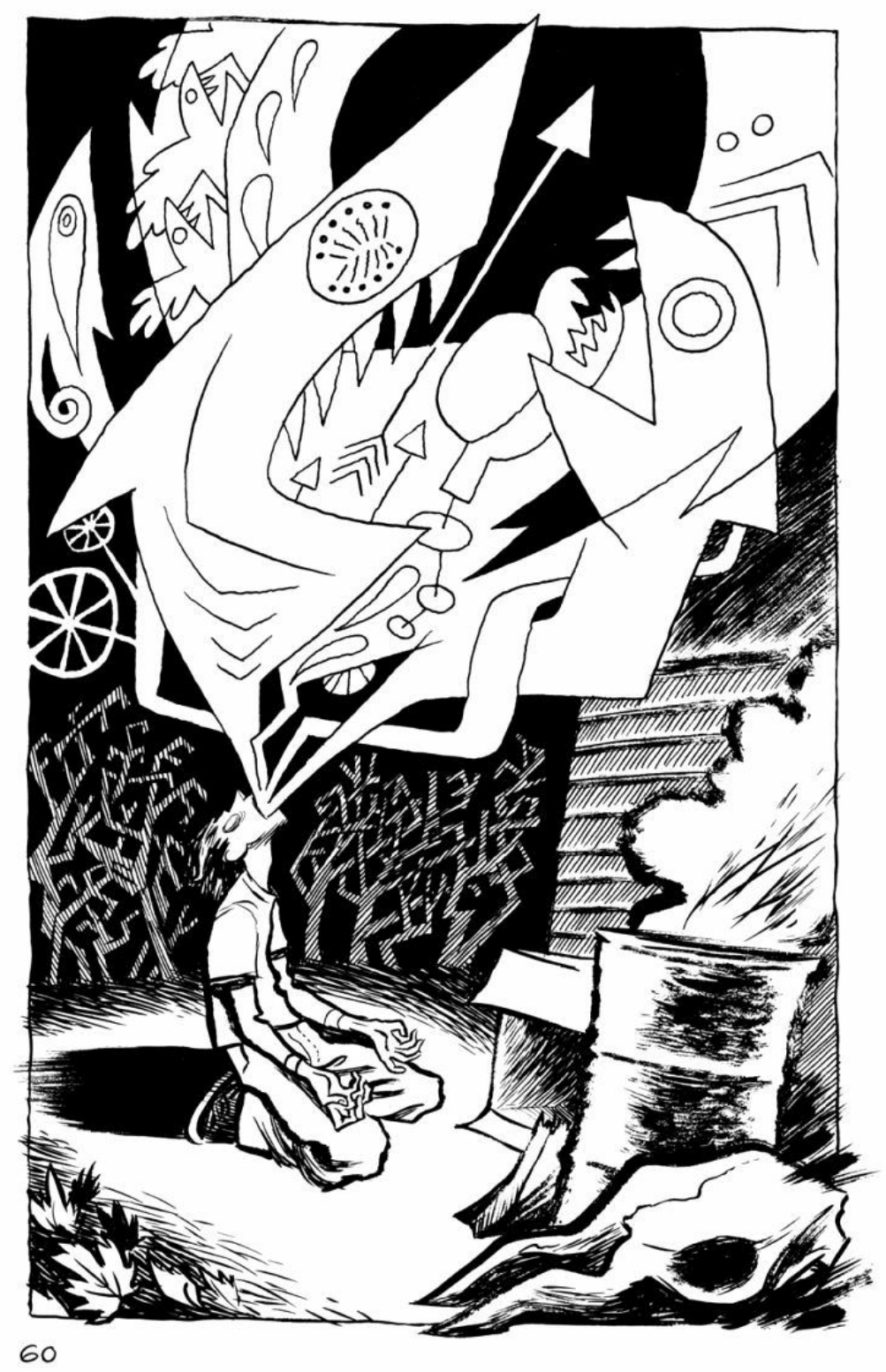

Figure 15: Thompson, Blankets, 60.

In cases such as these, the reader is afforded an opportunity for an emotional response that is uniquely provided by the typical formal features of comics. The reader's emotional experience will not only be in response to the conceptual or propositional information communicated by the comics narrative in question, but she will likely also respond to her visual perceptual experience of the artwork. Emotions, being processes, 
typically involve, "a non-cognitive appraisal. I can be afraid without judging that there is a snake before me; I may merely register a curly stick-shape on the forest floor." ${ }^{224}$ In other words, psychological hermeneutical images do not only convey information through their role as representations, because they can condition affective appraisals in the reader even before she consciously registers the conceptual information that the images are meant to communicate. ${ }^{225}$

Stylistic choices are not only important when depicting characters, however. Artistic style can also affect the emotional tone of a work for the reader in other ways. A simple and cartoony style may suggest simple and predictable narrative content with a generally positive or neutral emotional tone. As Duncan and Smith put it, "Readers can develop expectations about story content and tone from the style of art before they even read the first panel. A clear line style is usually associated with a lighthearted adventure in which the heroes are sure to triumph over the bad guys."226 On the other hand, "an ugly (brut) art style is more likely to depict a pessimistic worldview." 227 The style of images can communicate emotional tone independently of their content.

In cases where art is used in this way, the emotional salience of the narrative is suggested through general artistic presentation, rather than only through the content of depictions. Stylistic choices involve "[augmenting] basic lines and shapes with more visual cues, such as textures, lighting and shading, symbols and letters, colors, and

${ }^{224}$ Robinson, Deeper Than Reason, 55.

${ }^{225}$ Because emotions are processes the reader will also cognitively monitor those initial affective appraisals and therefore will be unlikely to respond as she would if the visual experience she is having was not only a representation (see the example of the depicted funeral above).

${ }^{226}$ Duncan and Smith, The Power of Comics: History, Form, and Culture, 162.

${ }^{227}$ Duncan and Smith, 162. 
special effects, such as exaggeration [...] These cues may further facilitate people's interpretation of emotion representations." ${ }^{, 28}$ Artistic and stylistic elements can shape both readers' emotional experiences of a comic and their understanding of that comic's thematic meaning(s).

There is some evidence that general stylistic elements of artworks may contribute as much or more to the emotional experiences of readers than do more direct depictions of characters' emotions. One study found that perceptions of instability in static artistic images were associated with negative emotional states in perceivers, even more than the content of the images. "Although both explicit emotional depiction and perceived dynamics enable emotional attribution, the latter seems to represent the more powerful source of information. ${ }^{229}$ This further corroborates that artistic style and design elements can serve to influence the types of opportunities readers have to emotionally engage with a work.

The artistic style of a comic can also be used to contrast conventional narrative expectations in order to influence the expected emotional responses of readers. In cases like these, the narrative content and artistic style of a comic can "form a disjunctive interdependence that disturbs readers by upsetting their previously-held beliefs" ${ }^{\prime 230}$ and can "generate powerful, long-lasting emotional responses in readers." ${ }^{, 231}$ A work may use a dark and sketchy style in the telling of an emotionally upbeat narrative. Alternatively, a

\footnotetext{
${ }^{228}$ Ma, et al., "Guidelines for Depicting Emotions in Storyboard Scenarios," 4-5.

${ }^{229}$ Marina Pavlova, Arseny A. Sokolov, and Alexander Sokolov, "Perceived Dynamics of Static Images Enables Emotional Attribution,” Perception 34, no. 9 (2005): 1112.

${ }^{230}$ Frank L. Cioffi, "Disturbing Comics: The Disjunction of Word and Image in the Comics of Andrzej Mleczko, Ben Katchor, R. Crumb, and Art Speigelman," in The Language of Comics: Word and Image, ed. Robin Varnum and Christina T. Gibbons (Jackson MS: University Press of Mississippi, 2001): 99.

${ }^{231}$ Cioffi, 97.
} 
simple, bright, or cartoony style may be used to convey a narrative with dark or complex emotional themes. In such cases, "artists are not offering a comfortable world to escape into, ${ }^{, 232}$ but disjointed and emotionally complex and storyworlds.

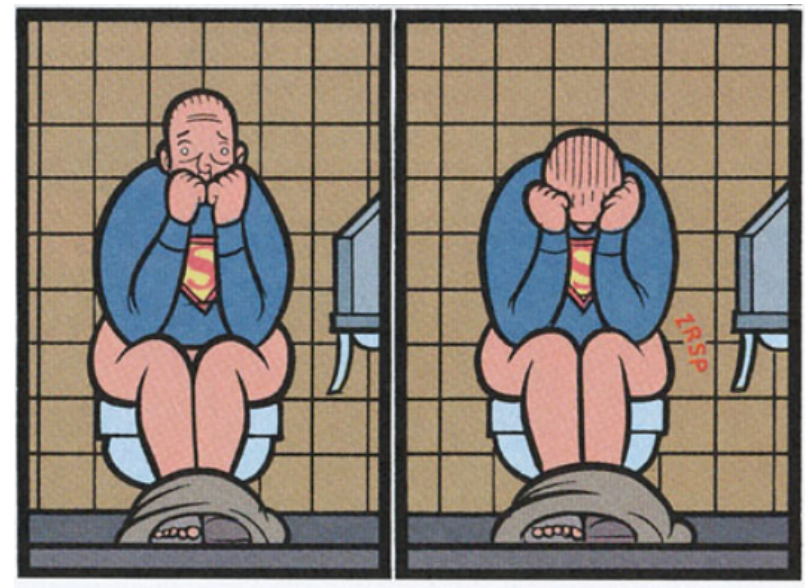

Figure 16: Chris Ware,

Jimmy Corrigan: The Smartest Kid on Earth, New York: Pantheon Graphic Novels, 2003.

For example, Chris Ware's graphic novel Jimmy Corrigan: The Smartest Kid on Earth exemplifies this potential for disjunction between narrative content and artistic style. The narrative focuses on the character of Jimmy, a middle-aged man with few friends, as he meets his father for the first time. The story traffics in the generally negative emotional themes of loneliness, isolation, boredom, and depression. However, Ware's artistic style is the stuff of children's cartoons. He uses an abstracted, cartoony, and clean-lined drawing style in order to create dissonance when accompanied by the emotionally weighty narrative. The effect of this disjunction between narrative content and artistic style is that the emotional weight of the narrative is particularly potent and may affect the reader long after she has finished reading the work. ${ }^{233}$

\footnotetext{
${ }^{232}$ Cioffi, 121.

${ }^{233}$ Cioffi, 99.
} 
Finally, design elements may also play into how the emotionally salient content of artwork in panels is experienced by readers of comics. With regard to the design and structure of panels, Thierry Groensteen articulates three parameters that are salient for the communication of the meaning of a panel. He writes, "The first two [features of panels] are geometric: they are the form of the panel (rectangular, square, round, trapezoidal, etc.) and its area, measurable in square centimeters [...] The third parameter, which is the site of the panel, concerns its location on the page and, beyond that, within the entire work." ${ }^{, 234}$ While Groensteen doesn't address the issue directly, these parameters have the ability to communicate emotional salience through panels by emphasizing certain narratively relevant information and/or minimizing other information.

Will Eisner also acknowledges some of the ways in which the design and placement of a panel manifests the panel's meaning and shapes the experience of the reader. He argues, "A narrow panel evokes the feeling of being hemmed in-confinement; whereas a wide panel suggests plenty of space in which to move-or escape [...] The shape of the panel and the use of perspective within it can be manipulated to produce various emotional states in the viewer."235 For example, a panel's larger size or centralized position on a comics page may emphasize the narrative importance, and subsequently increase the perceived emotional relevance, of the content or artistic style of that panel. The size, shape, placement, and perspective of panels aid the reader in understanding the emotional tone and feeling of the storyworld of a comic.

${ }^{234}$ Groensteen, The System of Comics, 28.

${ }^{235}$ Eisner, Comics and Sequential Art, 92. 
Closure, Imagination, and Emotion

With the previous discussions about character depiction and artistic style and design behind us, I will now discuss how I take these elements of reader experiences of comics to hang together. Having discussed the process of closure in detail in the previous chapter, only a few words on closure are necessary at this point in the discussion of readers' potential emotional engagements with comics. Because the typical formal features of comics necessitate the enactment of closure, McCloud goes as far as to say "closure is comics!" ${ }^{236}$ Closure relies on the interaction between the reader and the comic by requiring the reader to imagine and infer a coherent storyworld, drawing on her previously held experiences and expectations.

As Duncan and Smith write, "The reader performs an ongoing construction of meaning by considering each panel in direct relationship to the immediately previous panel and in the context of all previous panels. ${ }^{237}$ In this way, comics stories presume that readers will approach the work with certain background knowledge and the ability to understand and extract an appropriate meaning (or range of meanings). Because this type of engagement is required, the process of authoring a comic is "reductive" 238 in that the author must whittle down her story into a limited set of panels (that is, discreet depictions) that she considers adequate. The process of reading comics, on the other hand, requires readers to enact closure, which is "additive,"239 because readers imagine and infer elements of the narrative that are not directly depicted or described.

\footnotetext{
${ }^{236}$ Cioffi, "Disturbing Comics," 67.

${ }^{237}$ Duncan and Smith, The Power of Comics: History, Form, and Culture, 166.

${ }^{238}$ Duncan and Smith, 154.

${ }^{239}$ Duncan and Smith, 154.
} 
Emotional responses may be conditioned on the reader's enactment of closure (inference and imagination), but emotional responses may also be conditioned on inference or imagination alone. Also, a reader's emotional responses to the artistic style or design elements may shape how the reader enacts closure by affecting her imaginative engagement with the work. The figure below (Figure 17), depicts what I take to be the typical interrelations of the features of a comic (that is, style/design elements and artistic/textual content) and the elements of reader experiences that I have discussed in detail (that is, inference, imagination, closure, and emotional responses). There are, then, many ways for the elements of a comic to shape readers' cognitive and emotional engagements with the work.

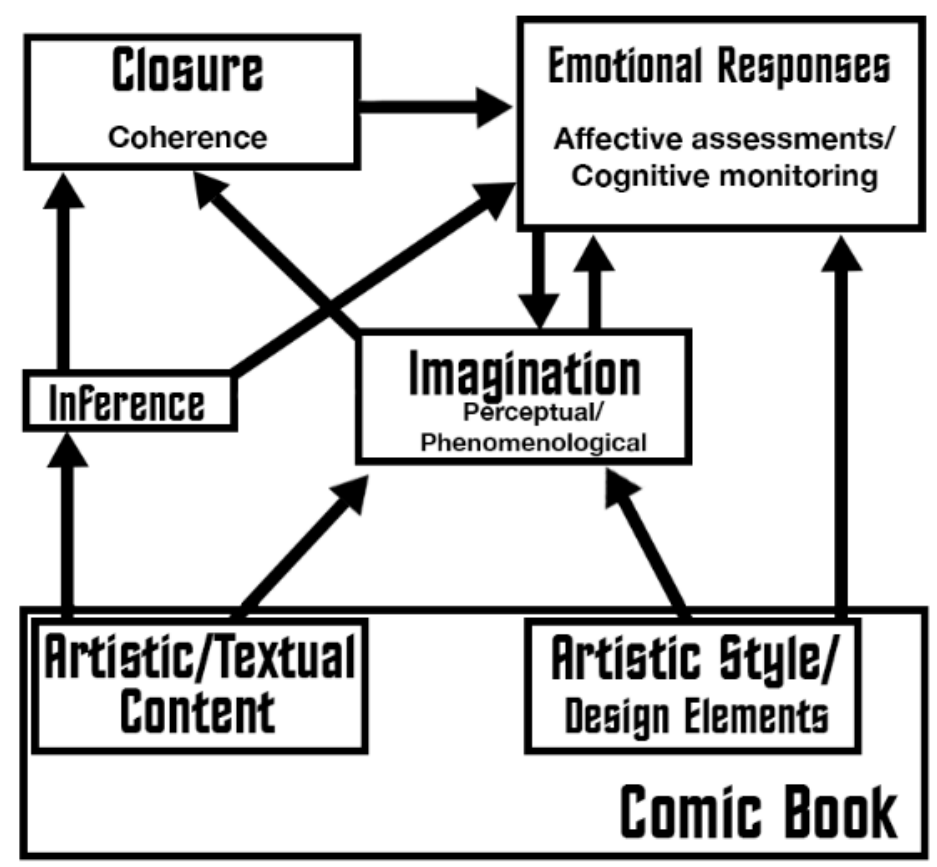

Figure 17: Relations of comic books to emotional and cognitive elements of reader experiences

In order to examine some of the possible ways in which the comic can shape reader experience, I will examine the sequence below (Figure 18). This sequence appeared in an annual holiday special issue of The Batman Adventures, a comics title in 
the style of the popular 1990's animated Batman television series. In this sequence, the villain Clayface clears his throat and spits wet clay mud onto the guns of two police officers, who are attempting to arrest him. There are many ways in which reader engagement with this sequence could proceed.

The artistic content allows us to infer that the brown splatters in the fourth panel of the sequence are the same clumps of matter that Clayface is depicted as spitting out in the third panel and we imagine that material flying through the air in the intervening narrative time between panels three and four, thus enacting closure. We also imagine the sounds that arise from this interaction, with the guidance of the onomatopoetic terms incorporated into the depiction. The artistic style of these onomatopoetic terms may influence the way in which we imagine the sounds, with "HAWCCHH" being sloppy and hollow and "PTOO!" being sharp and staccato.
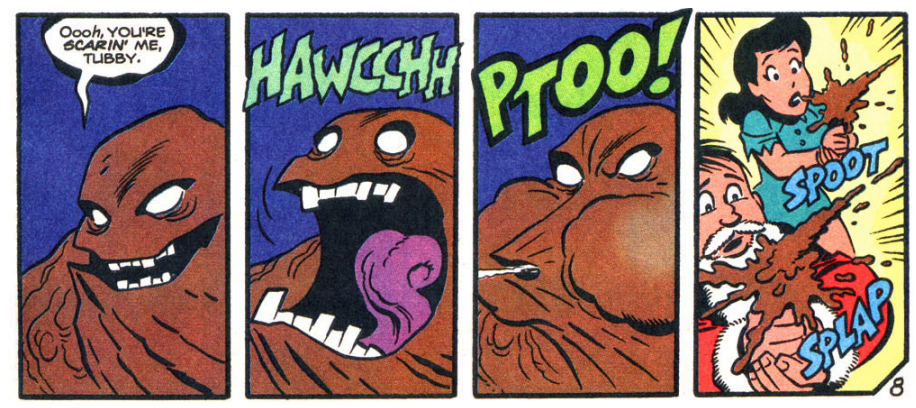

Figure 18: Paul Dini and Bruce Timm, "Jolly Ol' St. Nicholas," The Batman Adventures Holiday Special \#1

(New York: DC Comics, 1995), 8.

The reader may emotionally engage with this sequence, as well. For example, she may feel some amount of disgust from imagining the goopy brown matter launched from the throat of the clay villain. Such an emotional disgust response would be conditioned upon the enactment of closure, when the reader infers the identity of the brown matter in panel four and imagines its texture and viscosity. The reader may experience an affective appraisal of her own imagined understanding of the clay phlegm. 
Alternatively, the cartoony, bright, and simple artistic style with which the content of the sequence is communicated may lead the reader to have an emotional response of lightheartedness, rather than disgust. This emotional response, in turn, may affect the reader's imaginative engagement (that is, she may imagine the movement/sound of the phlegm in a hyper-realistic style) and, thereby, her enactment of closure will be affected by her emotional response. For example, she may imagine the phlegm as moving at a cartoonishly slow speed through the air toward the police officers' guns and, thus, she will understand the coherence of the storyworld differently than if her emotional response had been that of disgust. A reader may also infer and imagine the events communicated by this sequence without any resultant emotional response, however. Emotions may arise as a result of the enactment of closure (or inference or imagination alone), but closure does not necessarily involve an emotional response from readers.

Closure is not a hermeneutically unconstrained process and appropriate interpretations of a given work may fall within a potentially narrow range of appropriate meanings. ${ }^{240}$ The constraint of closure arises because panels in comics function as "attention units" and "windows" into the world of the narrative. ${ }^{241}$

Panels $[\ldots]$ can simulate what our vision would be like if we were watching a scene in person. This creates a sensation that panels facilitate a 'spotlight' that reveals only portions of a larger environment. In actuality, these glimpses create the whole view of the environment in the mind of the reader. These various panels represent parts of the scene, which allows us to inferentially [and imaginatively] construct a full understanding of the broader scene. ${ }^{242}$

A reader's previous emotional state, expectations, and affective dispositions will affect her experiences of the emotional salience and tone of scenes depicted in a comic

\footnotetext{
${ }^{240}$ See the discussion of polymorphic panels in the previous chapter.

${ }^{241}$ Cohn, The Visual Language of Comics, 58.

${ }^{242}$ Cohn, 59.
} 
because closure necessitates that readers actively imagine and infer, bringing their own interpretation to the text. However, the range of potential understandings will be influenced by the content depicted in panels, which can function to simulate attentional shifts in the reader's perspective on the storyworld. For example, the emotion of pity toward Clayface would be an unlikely and inappropriate response to the above sequence because of the narrative events depicted.

Noël Carroll notes that in the context of "mass fictions, the emotions keep us focused on the plot on a moment-to-moment basis. They organize our attention in terms of what is going on in a scene, and they also prime our attention to the kinds of things to expect in future scenes. ${ }^{243}$ So, the guiding of reader attention is often conditioned upon the ways in which panels are constructed and the narratively relevant information that their structuring serves to emphasize, while the resulting reader awareness of the emotional importance of that information helps to ensure continued reader engagement and to condition expectations for the remainder of the narrative. Emotional engagement often keeps us reading, but understanding what events are represented is accomplished through closure, which itself may be influenced by our emotional engagement.

While other narrative mediums (such as, theatre and film) can require imaginative work that is similar to closure, comics require inferential and imaginative work on a more fine-grained level, as discussed in detail in the previous chapter. Again, closure in comics requires readers to infer and imagine all motion and duration. Therefore, the way that readers typically engage with comics, because of their formal features, can determine the emotional impact of various parts of the comics narrative.

${ }^{243}$ Carroll, A Philosophy of Mass Art, 249. 


\section{Conclusion}

In this chapter, I have argued that comics offer their readers unique and mediumspecific opportunities for emotional engagement, with emotions being understood as processes involving affective appraisals, physiological shifts, and cognitive monitoring. The typical formal features of comics and their implications for reader engagement allow for emotional experiences in ways that are distinct from fully textual works, visual artworks, or films. Comics allow us unique opportunities for responding emotionally to their stories because of the unique perceptual and cognitive experiences that result from the ways we read comics. Comics afford emotional experiences through character depiction, artistic style and design, and the need for closure.

The mimetic nature of character depiction can provoke emotional contagion, empathic, and sympathetic responses, while criterial prefocusing can shape readers' perspectives, resulting in various emotional experiences. Both artistic style choices and design elements can shape the emotional experiences of the reader by setting the emotional tone and expectations about the narrative even before she engages with the representative content of the narrative. Finally, the active reader engagement required by the process of closure, explored in detail in the previous chapter, entails that readers' outside knowledge and preliminary expectations can influence her emotional response to the narrative. Also, what she imagines and infers about the storyworld can shape her emotional engagement with the comic, and her emotional responses to the comic may influence how she imagines the storyworld. That is, the relationship between closure and emotional responses is bi-directional (via imagination). ${ }^{244}$

${ }^{244}$ See Figure 17. 
The experiences afforded to the reader by comics do not only shape the emotional tone of the content presented, but also implicitly encourage certain readings that content. In the chapter three, I will discuss the role that such implicit readings can play in regard to the understanding of the nature of selves. Readers enact closure in order to understand the content of comics narratives and the formal features with which those readers engage shape their emotional responses, as we have seen. The influence of these typical formal features on emotional and cognitive reader experiences implies a particular and potentially culturally subversive vision of the nature of selves. I will argue that the nature of the depictions of characters in comics implicitly offers readers a view of selves as narrativized and conventional, subverting the more common Western view of selves as unified, essential, and static. 


\section{III: COMICS AND THE DISSOLUTION OF THE TRADITIONAL SELF}

\section{Traditional Understandings of Self}

Now that I have discussed the role of cognitive and perceptual processes in the enactment of closure in the first chapter and the unique opportunities for emotions in relation to comics in the second chapter, I now turn to the question of the implications of comics for the concept of selfhood. It is at this point that my focus will begin to shift from the predominantly theoretical, philosophical, and ostensibly ahistorical issues arising from the formal features of comics to a more contextual examination of their place in culture. The U.S. cultural landscape, since the rise in popularity of comics in the early Twentieth century, will contextualize and illuminate the potential significance of the understandings of selfhood implied by the types of stories that have been typical of comics, as well as the formal features I have examined thus far.

Comics in the U.S. cultural context have been and are consumed against a backdrop of particular ideas about and conceptions of selfhood that have arisen from social, religious, economic, and cultural factors. The ways in which comics typically depict characters, as well as the broader types of reader experiences afforded by comics, frequently offer readings of selfhood that are distinct from and undermining of more common Western notions of selves as unified, enduring, and essential entities. This implication of the comics form is culturally relevant regardless of the question of the 
actual nature of selfhood, a question that I will bracket for the purposes of this work. The fact that an alternative, and potentially subversive, interpretation of selfhood is implied to varying degrees in U.S. comics will be particularly relevant when exploring the relationship of the comics medium to esoteric and occultist religiosities and traditions in the final chapter.

Many cultural factors have led to the traditional Western notion that selves are unified and enduring essences of human identity, including the prominence of highly individualist political philosophies and economic shifts toward industrialization and specialization. Another factor in this entrenched view of selfhood is the influence of the concept of the "soul" within the Christian tradition. ${ }^{245}$ Christianity has been highly influential in U.S. culture and tends to understand human selves as consisting of (at least in part) eternal souls, which constitute the unified essence of a person (that is, the person's identity). "The Western self largely acquired its basis in undividedness-in oneness-through [the influence of] medieval Christian theology."246 This traditional conception of the self as being constituted by a single essential entity that remains stable

${ }^{245}$ C.S. Lewis, The Weight of Glory (New York: HarperOne, 1980): 46. The conceptual ties between the religious conception of a soul and the high valuation of individual selves (conceived of as unified essences) can be seen in the popular theological writings of C.S. Lewis, one of the most influential apologists for the Christian tradition in the West in the Twentieth century. He argues, "there are no ordinary people. You have never talked to a mere mortal. Nations, cultures, arts, civilizations - these are mortal, and their life is to ours as the life of a gnat. But it is immortals whom we joke with, work with, marry, snub and exploit - immortal horrors or everlasting splendors." Human selves, in this religiously resonant view, are understood to be the unified subsisting essences of humans' identities that will outlive their social and cultural contexts.

${ }^{246}$ A. David Lewis, American Comics, Literary Theory, and Religion: The Superhero Afterlife (New York: Palgrave Macmillan, 2014): 10. 
across the lifespan of an individual frames the core of human persons as ontologically robust, continuous, and unified. ${ }^{247}$

This view of selves as essential, unified, and continuous holds enormous sway even in Western discourses that are non-religious. Some, including psychologist Paul Bloom, go so far as to argue that humans are "commonsense" dualists. ${ }^{248}$ Some, such as Bloom, argue that humans innately operate on the belief that we have bodies but our identities consist of indivisible immaterial souls. Bloom writes, "We don't feel as if we are bodies. We feel as if we occupy them." ${ }^{249}$ In other words, this would suggest that some people tend to identify with their soul or self, to the exclusion of their body, and the two are seen as distinct. Science writer Michael Shermer notes that people betray their belief that they consist of an essential self when they 'speak of 'my body,' as if 'my' and 'body' are two different entities." ${ }^{250}$ Whether this view is simply culturally conditioned

${ }^{247}$ John Barresi and Raymond Martin, "History as Prologue: Western Theories of the Self," in The Oxford Handbook of the Self, ed. Shaun Gallagher (New York: Oxford University Press, 2011): 37. The belief in selves as immutable and unified essences of human identity is highly compatible with common iterations of Western religious traditions in part because of the pivotal role of afterlife beliefs in many Western religious systems. "In today's Western industrialized cultures many people are not only curious about whether they themselves will survive, but long to survive, particularly in some way that is better than their earthly lives. Such attitudes [...] seem to owe their prevalence, if not their very origin, to Christianity." While religiosity may have been one of the primary sources of this entrenched view of selfhood, the tendency of Western post-industrial cultures to rhetorically privilege the concerns and perspectives of individuals politically and economically has fostered an environment that is particularly suited to this conception of selfhood.

${ }^{248}$ Paul Bloom, Descartes' Baby: How the Science of Child Development Explains What Makes Us Human (New York: Basic Books, 2004): xiv.

${ }^{249}$ Bloom, Descartes' Baby, 191.

${ }^{250}$ Michael Shermer, The Believing Brain: From Ghosts and Gods to Politics and Conspiracies---How We Construct Beliefs and Reinforce Them as Truths (New York: St. Martin's Griffin, 2011): 128. 
or somehow likely given humans' typical psychobiological makeup, the conception of selves as unified essences is prevalent in the Western context. ${ }^{251}$

Reliance on the notion of an essential self is also illuminated through cultural practices revolving around the assigning of value, responsibility, praise, and blame. ${ }^{252}$ Blaming Jill for anti-social actions in her past tends to entail the presupposition that there is a unity to Jill in the present and Jill in the past. That is, reward and punishment are often framed in terms of there being a self that is stable across time, such that my blame or praise in the present suitably falls on the same self that engaged in the relevant actions in the past. This implicit view is on display from political philosophies that heavily emphasize (and sometimes overestimate) the role of the personal responsibility to domestic disagreements about whose turn it is to take out the trash.

Evidence of the ubiquity of the unified and essential self-view abounds in U.S. popular culture, as well. Consider the common science fiction trope of the body swap. In the original 1960's Star Trek television series, an episode entitled "Turnabout Intruder" revolves around the conflict that occurs after Dr. Janice Lester, a former friend of Captain

${ }^{251}$ While I lean toward the position that this type of dualism is culturally conditioned, I do not take the arguments made here to turn on whether this belief is culturally conditioned or innate, given typical psychological development. Regardless of the specific factors that condition this view of the nature of selves, the perspective has been pervasive in the century since the development and growth in popularity of comics.

${ }^{252}$ Paul Bloom, "Natural-Born Dualists," Edge, May 11, 2004, https://www.edge.org/conversation/natural-born-dualists. "Our dualistic perspective also affects how we think about such moral and political issues as stem-cell research, abortion, animal rights, and cloning. These are complicated issues, but the way people tend to address them — often explicitly, but always implicitly — is in terms of the question: Does it have a soul? If so, then the being in question is worthy of protection, a precious individual. If not, it is a mere thing." 
James T. Kirk, enacts a "life energy transfer" on him. ${ }^{253}$ The effect is that Kirk is trapped in Lester's body, and vice-versa. Star Trek is not the only example of this narrative trope. ${ }^{254}$ The body swap is probably most widely recognized from its use in the film Freaky Friday, ${ }^{255}$ in which a mother and daughter switch bodies and ultimately both gain a new level of empathy for the struggles of the other over the course of the story. The use of this trope in pop culture narratives speaks to its compatibility with entrenched intuitions about the self and the possibilities for the activities of selves in the world, beyond only religious discourses.

While I have argued that the traditional Western conception of selfhood often includes the properties of unity and essentiality, the popular notion of the self (being a folk notion) is not necessarily a consistent or nuanced concept. It may be unclear, even to those who endorse the idea of an essential self or soul, what this type of self consists of and how it relates to the mind of the individual. Philosopher Owen Flanagan writes:

In the standard view, a soul comprises a person's essence and is not itself a part of the natural fabric of things. [...] Two possibilities stand out. Either the mind has a pure immutable extra ingredient, its essence, $[\ldots]$ that constitutes something like its pure, permanent, structural form-something it possesses, indeed that it is, prior to getting entangled with the world and that can survive that entanglement after death. Or, a less parsimonious idea is that there is, in addition to mind, a soul-a third, extra ingredient that contains the incorporeal mind and that possesses the properties of indivisibility and immutability. ${ }^{256}$

${ }^{253}$ Star Trek: The Original Series: Season Three, directed by Herb Wallerstein (1969; CBS Paramount International Television, 2008), DVD.

${ }^{254}$ However, its use in Star Trek is particularly relevant in displaying the commonality of this trope beyond arenas of religious discourse, especially given the anti-religious tendencies of Star Trek creator Gene Roddenberry.

${ }^{255}$ Freaky Friday, directed by Gary Nelson (1976; Walt Disney Pictures), DVD. ${ }^{256}$ Owen Flanagan, The Problem of the Soul: Two Visions of Mind and How to Reconcile Them (New York: Basic Books, 2002): 162-163. 
Regardless of the potential lack of philosophical specificity of the traditional Western conception of selves, a general belief in "some permanent and abiding part of myself that makes me me, and which in addition may survive my death-is widespread." ${ }^{257}$ In this entrenched view, the essential self is thought to stay "the same amid the flux and [make an individual] the same person over time. ${ }^{258}$ This understanding of selfhood that is thought to involve essentiality, continuousness, and unity over the lifespan of the individual is most relevant to the points argued below.

\section{Self as Narrative}

The term "self" is used in various ways in both theoretical and popular discourses. One popular position, as I just detailed, holds that selves are constituted by unified essences that subsist across the lifespan of an individual. Regardless of its accuracy or inaccuracy, this view shows the influence of the political, economic, and religious contexts in which it has developed. But while it has been the entrenched view in the West, individually and also institutionally, other conceptions of selfhood have been available for millennia.

For example, the denial of an essential subsisting self (referenced by the term "anātman") is one of the foundational tenets of the Buddhist religious tradition. ${ }^{259}$ Additionally, some European enlightenment philosophers, including Hume, denied the existence of an essential self. ${ }^{260}$ A more recent recurring philosophical articulation of selfhood is the view that selves are constituted, in some sense, by narratives. An

\footnotetext{
${ }^{257}$ Flanagan, The Problem of the Soul, 164.

${ }^{258}$ Flanagan, The Problem of the Soul, 165.

${ }^{259}$ Donald W. Mitchell, Buddhism: Introducing the Buddhist Experience (New York: Oxford University Press, 2002), 37.

${ }^{260}$ Barresi and Martin, "History as Prologue," 40-43.
} 
understanding of narrative views of selfhood will help to spell out the implications for the views of selves implied by the typical formal features of comics.

I will focus on narrative views of selfhood, as I take these views to best illuminate the implications that the form of comics holds for framing the concept of the self.

However, two brief caveats are in order. First, there are a variety of philosophical views about selfhood that I will not explore in detail in this chapter. ${ }^{261}$ While some of these views have merit with regard to exploring the first-personal nature of subjectivity, I will be primarily addressing issues of the coherence of selves over time and representations of selves over time (both narrative and actual time), and therefore the conception of selves as narratives will be most relevant to this discussion.

It is also important to note that I am not here making an argument that narrative views of selfhood are accurate. I am not directly arguing for the narrative self as a generalizable view of the ontological nature of selves. ${ }^{262}$ Rather, I am arguing that comics (by virtue of their formal features) implicitly support the narrative view of selfhood and this implication influences the types of stories most commonly associated with them. The narrative view of selfhood could be inaccurate and the formal features of comics would still offer readers an implicit affirmation of selves as narratives. This is a culturally

${ }^{261}$ Shaun Gallagher and Dan Zahavi, The Philosophical Mind (New York: Routledge, 2008): 226; Dan Zahavi, Subjectivity and Selfhood: Investigating the First-Person Perspective (Cambridge, MA: MIT Press, 2005): 334. For example, philosophers in the phenomenological tradition have made the case that the self is "an integral part of the structure of our conscious lives. More precisely, [the self] possesses experiential reality and is in fact identified with the first-personal character of the experiential phenomena." This entails that there is a "minimal sense of self" that is not constituted by a unified essence or a temporally extended narrative, but is constituted by a quality of "mineness" inherent to subjective experience.

${ }^{262}$ To put my cards on the table, I do adhere to the view that selves are constituted by narratives in one way or another. However, the arguments contained herein should stand whether or not I am right to hold this view. 
relevant, interesting, and mostly unexplored implication of the form of comics regardless of what the actual nature of selfhood turns out to be. In other words, I am exploring the cultural implications of a particular philosophical view about the self, rather than offering an account of the actual nature of selves.

It is important to note that the narrative view of selfhood has some prominent detractors, as well. For example, philosopher Galen Strawson strongly criticizes the narrative view of selves, arguing that many people do not experience their lives as narrative in any way, nor should they. ${ }^{263}$ A thorough response to Strawson's critiques of the narrative view is beyond the scope of this work, but more importantly his critiques will be largely extraneous to the following discussions of representations of selfhood and the potential implications for reader experiences. ${ }^{264}$ Once again, the actual truth of the narrative view of selves will not be defended here. Rather, a thorough explanation of some common versions of this view will make clear the ways in which the formal features of comics imply narrative selfhood and undermine the entrenched Western essentialist view.

Philosopher Daniel Dennett offers a version of the narrative view of the self that tracks particularly well with the implications of the comics medium. Dennett argues that

${ }^{263}$ Galen Strawson, “Against Narrativity,” Ratio 17, no. 4 (2004): 428-52.

${ }^{264}$ Strawson, 430. To some extent, my claims about the potential for comics to affect reader experience may dovetail nicely with Strawson's notion of "episodic" life, in that the self-narrative is not a moment-to-moment necessity of the experience of people. People may have experiences (at least temporarily) of themselves as having no narrative extension into the past or future. In fact, it is an interesting theoretical possibility that anomalous experiences of self-loss could potentially be described as a first-personal shift from a "diachronic" to an "episodic" perspective. I am not committed to this view, but it raises interesting questions for potential future work. 
a typical self consists of what he calls a "center of narrative gravity." ${ }^{265} \mathrm{He}$ analogizes selves to the concept of centers of gravity within the study of physics, claiming that both are purely abstract objects or "theorist's fictions," ${ }^{266}$ which are not part of the world in the same way that physical objects are. A center of gravity, Dennett notes, "is not one of the real things in the universe in addition to the atoms. But it is a fiction that has nicely defined, well delineated and well behaved role within physics." ${ }^{267}$ Similarly, he claims that selves are not real things in the universe, but are theorist's fictions, helping us to explain and predict the actions, intentions, and dispositions of others and ourselves.

Selves are, in Dennett's view, heuristic tools that aid in our understanding of certain events in the physical world.

In defending the notion of selves as narrative "abstractums," Dennett constructs a thought experiment about a novel-writing robot. The machine is equipped with artificial intelligence, mobility, and the ability to print sentences, which constitute its novel. However, there is no question that the robot is just "a dumb machine."268 This mobile novel-writing machine begins its novel with the sentence "Call me Gilbert"269 (a nod to Melville's Ishmael). Over time, the novel that the machine continues to write contains elements in which "Gilbert" undergoes events highly similar to those undertaken by the robot, making "the fictional career of the fictional Gilbert [bear] an interesting

${ }^{265}$ Daniel C. Dennett, "The Self as a Center of Narrative Gravity," in Self and Consciousness: Multiple Perspectives, eds. Frank Kessel, Pamela Cole and Dale Johnson, (New York: Psychology Press, 1992): http://cogprints.org/266/1/selfctr.htm.

${ }^{266}$ Dennett, "The Self as a Center of Narrative Gravity," http://cogprints.org/266/1/selfctr.htm.

${ }^{267}$ Dennett, http://cogprints.org/266/1/selfctr.htm.

${ }^{268}$ Dennett, http://cogprints.org/266/1/selfctr.htm.

${ }^{269}$ Dennett, http://cogprints.org/266/1/selfctr.htm. 
resemblance to the 'career' of this mere robot moving through the world." ${ }^{270}$ Thus, the abstractum of "Gilbert" (which is not part of the contents of the physical world) can be used as a hermeneutical or interpretive tool that can aid observers in explaining, understanding, and predicting the behaviors and dispositions of the novel-writing robot (which is part of the physical world). When the history and behavior of the robot matches that of Gilbert, we may rightly come to interpret the robot as being (or having constructed a self named) "Gilbert."

Human beings, in Dennett's view, make similar hermeneutical moves when attempting to interpret and predict the behaviors and thoughts of others and themselves. The human organism is constituted by multiple physical systems that are "somewhat disunified"271 and can present potential problems for interpreters who are attempting to make sense of the organism's activities. Even in typical cases that are unproblematic in terms of understanding the self, interpretation is still necessary. For example, why did I react so angrily when Bob asked about my new job? Perhaps I understood Bob's question to be a veiled insult because my new job is less prestigious than my previous one. In a typical case like this, interpretation is used to maintain an explanatory coherence in regard to one's activity via the self-narrative. ${ }^{272}$

\footnotetext{
${ }^{270}$ Dennett, http://cogprints.org/266/1/selfctr.htm.

${ }^{271}$ Dennett, http://cogprints.org/266/1/selfctr.htm.

${ }^{272}$ J. David Velleman, Self to Self: Selected Essays (New York: Cambridge University Press, 2006): 206, 211-17, emphasis mine. One criticism of Dennett's view should be addressed. Philosopher J. David Velleman accepts most of the features of Dennett's account of selfhood, but diverges from Dennett by claiming that self-narratives (in most cases) are "both fictive and true." Velleman accepts that selves are abstractums (that is, they are not part of the furniture of the world), but argues that the self-narrative of an individual does more than simply interpreting and predicting the thoughts and actions of an individual. Rather, one's self-narrative can influence one's future actions and tendencies in order to allow one to maintain a coherent self-narrative. On this view, the
} 
One feature of selves that plays a role in Dennett's view that they are theorist's fictions, rather than substantive entities, is that selves do not necessarily adhere to the principle of bivalence. ${ }^{273}$ In other words, some perfectly intelligible questions about selves may not have determinate answers, while other questions about any particular self, or more precisely about the particular organism with which the self is associated, may have strictly determinate answers that are not open to interpretive adjustments.

For example, “is Will Simpson left handed?” (No, by the way). However, other questions may have no answer or the answer may be developed over time through the interpretation enacted by the individual organism with which the self is associated (potentially in collaboration with other interpreters) only after and potentially because the question has been posed. For example, what does Will Simpson believe is necessary for true friendship? This question is potentially indeterminate. While I may spend time thinking about friendship and devise a potential response that answers the question

self-narrative functions in a more robust way than providing simple prediction and interpretation of one's behavior and tendencies. One's self-understanding often serves to partially determine one's future trajectory in the service of maintaining the ability to coherently self-interpret. The ontological nature of self-narratives only concerns the arguments put forth here insofar as selves are, on the traditional Western view, seen as essential entities that exist as part of the furniture of the world. Regardless of whether they are true, false, fictional, or non-fictional, the relevant implication of both Dennett's and Velleman's views is that selves are actively constructed narratives that help to recount, interpret, predict, and (potentially) determine the temporal trajectories of the thoughts and actions of physical organisms. I will continue to refer to selves as theorist's fictions when discussing this narrative view of selfhood, although I do not mean necessarily to reject Velleman's contention by doing so. His contention about selfdetermination is simply extraneous to this line of argumentation.

${ }^{273}$ Arkady Bolotin, "The Quantum Pigeonhole Principle as a Violation of the Principle of Bivalence," Quantum Studies: Mathematics and Foundations (2017): 1-6. There may be fundamental physical forces or particles about which the question of the applicability of the principle of bivalence is uncertain. However, this issue should have no bearing on the discussion at hand, which focuses on physical systems at the scale of everyday human activity. 
sufficiently, there is not a determinate answer in the absence of my (or others') interpretive engagement with the question. Perhaps I will live my entire life and never consciously develop a coherent position on the requirements for friendship.

Similarly, fictional characters can also evade the principle of bivalence. At what SHU measurement on the Scoville spiciness scale does food become too hot for Batman to enjoy? Clearly, there is no fact of the matter because Batman is a fictional character and the various writers of the Batman character (to my knowledge) have never addressed this question in a definitive way. However, the question of what city Batman lives in does have a determinate answer (that is, Gotham City). There are determinate and indeterminate elements to fictional characters and there are also such elements to actual selves in the world. Both fictional characters and the theorist's fiction of the self, in this view, are abstractums. They are useful tools for understanding and navigating the world, but they are not part of the immutable furniture of the world apart from our interpretations.

It is important to note that in Dennett's view, the usefulness of a self is that it imparts interpretive, predictive, and explanatory power regarding the organism with which the self is associated. The self is not simply a fiction, but is a theorist's fiction. Selves are not infinitely malleable and interpretations of them are contingent on certain unalterable facts (such as, physical and historical facts about the organism or its environment). Dennett writes:

We cannot undo those parts of our pasts that are determinate, but our selves are constantly being made more determinate as we go along in response to the way the world impinges on us. Of course it is also possible for a person to engage in auto-hermeneutics, interpretation of one's self, and in particular to go back and think about one's past, and one's memories, and to rethink them and rewrite them. This process does change the 'fictional' character, the character that you are $[\ldots]$ 
This would be an utterly mysterious and magical prospect (and hence something no one should take seriously) if the self were anything but an abstractum. ${ }^{274}$

So, it is the self's lack of substantive ontology that permits the range of flexibility available in self-interpretation, a common human activity.

I do not take the arguments presented here to hang on the understanding that selves are the same, in all relevant respects, as fictional characters in narrative media. That position would be untenable, as there are clearly significant differences. For example, the ways and extents to which fictional characters are indeterminate are distinct from the ways in which selves are indeterminate. The physical facts regarding an organism with which a self is associated are always subject to the principle of bivalence. That is, there is always a fact of the matter. Dennett notes this by writing that, "with regard to any actual man, living or dead, the question of whether or not he has or had a mole on his left shoulder blade has an answer, yes or no." ${ }^{, 275}$ This is not the case for fictional characters in narrative media. Asking which arm has been itchier over the course of Superman's life is not simply a practically unanswerable question. There is no fact of the matter to be discovered, regardless of how much information we possess about the world. ${ }^{276}$ However, what is relevant for our purposes is that both fictional characters and actual selves necessitate, and are constituted by, interpretations (that is, projected narration in order to be understood as a subsisting character) within constraints (that is,

${ }^{274}$ Dennett, "The Self as a Center of Narrative Gravity," http://cogprints.org/266/1/selfctr.htm.

${ }^{275}$ Dennett, http://cogprints.org/266/1/selfctr.htm.

${ }^{276}$ It should be noted that I am not aware of any history of representations of Superman's arm itchiness. But even if there is a fact about which arm Superman has been represented as scratching more, or some other potential textual evidence, this fact can change with the publication of new Superman material. And various Superman narratives may contain mutually exclusive claims about which arm he has found itchier. 
established truths about the actual or fictional world of which the relevant self is a part). ${ }^{277}$

Dennett's account relies on a clear distinction between the narrative of the self and the organism with which the self is associated. One's self is not one's body and questions about one's self are not necessarily questions about one's body. Rather, "the chief $[\ldots]$ character at the center of [one's] autobiography is one's self. And if you still want to know what the self really is, you're making a category mistake." ${ }^{278}$ In this view, the self is an interpretive narrative achievement typically enacted principally by the organism with which it is associated, but potentially constructed by or in conjunction with others who are involved in interpreting the activity of that organism.

Philosopher Marya Schechtman offers an account of selfhood that also frames the self as a narrative construction. However, her view is distinct from Dennett's in that while he understands the self as a fiction, Schechtman "sees the self as real and constituted by a narrative." 279 The question of whether or not the self is "real" in this sense should not be a determining factor for the strength of the claims I will make throughout the remainder of this chapter. For my purposes, the relevant and shared aspects of both Dennett's and Schechtman's views is that selves, regardless of their ontological categorization, have a narrative form, are actively constructed, and "are not

${ }^{277}$ Metzinger, "The No-Self Alternative," 283. This view mirrors the ontological antirealism of philosopher Thomas Metzinger, who argues, "we just don't find a substantial self anywhere in the world and nothing on the level of scientific facts determines our metaphysics in this way." On this type of anti-realist view, selves are not included in the totality of entities in the world, but selves are a common feature of discourse that helps with the project of interpreting and predicting the actions and motivations of certain systems within the world (e.g., certain organisms).

${ }^{278}$ Dennett, http://cogprints.org/266/1/selfctr.htm.

${ }^{279}$ Marya Schechtman, "The Narrative Self," in The Oxford Handbook of the Self, ed. Shaun Gallagher (New York: Oxford University Press, 2013): 398. 
[physical or] metaphysical substances at all." ${ }^{280}$ Schechtman calls selves "everyday objects" and Dennett calls them fictional "abstractums," but these distinct determinations both entail that selves are not things in the physical world. Rather, they are conceptual achievements constituted by the interpretations and representations of events in the physical world by organisms, both individually and in community.

Schechtman calls her account of selves the "Narrative Self-Constitution View"281 (NSCV). The NSCV, like Dennett's view, distinguishes between the physical organism and the self-narrative that helps to conceptually frame the historical trajectory of the organism. She argues that, "the crucial thing to recognize is that the kind of life I am describing [that is, the life of a narrative self] is held together by the form of its unfolding rather than by its instantiation in a single human animal." 282 In other words, the self is not identical to the organism (or other physical processes) with which it is associated (similar to Dennett's distinction between Gilbert and the robot).

Like Dennett's view that selves are theorist's fictions, Schechtman's view requires that self-narratives involve interpretation within certain constraints. The NSCV requires that self-narratives "meet what it calls the 'reality constraint' and the 'articulation constraint, ${ }^{, 283}$ which are intended to ensure that self-narratives achieve the common practical requirements for full personhood. These constraints help to flesh out Schechtman's understanding of the self as a narrative that serves the practical needs of individuals and communities. The articulation constraint involves the ability to "articulate

\footnotetext{
${ }^{280}$ Marya Schechtman, Staying Alive: Personal Identity, Practical Concerns, and the Unity of a Life (New York: Oxford University Press, 2014): 180.

${ }^{281}$ Schechtman, "The Narrative Self," 398.

${ }^{282}$ Schechtman, Staying Alive, 109.

${ }^{283}$ Schechtman, Staying Alive, 101.
} 
one's narrative locally where appropriate," while the reality constraint "demands that our narratives fit with the basic conception of reality shared by those in our community.",284 Both of these features of Schechtman's NSCV are distinct from, but largely compatible with, Dennett's center-of-narrative-gravity view. The self is a narrative achievement typically realized by the organism with which the self is associated and the self-narrative is constrained by certain determinate facts about the organism, its history, its community, its environment, and its community's understanding of reality.

In the views discussed above, the traditional Western understanding of the self as unified, continuous, and essential, is a mistake. The self is a narrative interpretation of the activities exhibited by an organism (for our purposes in this section, a human organism). There is, according to self-narrative theorists, no evidence of the existence of an essential unified self, only the narrativizing of various physical events and processes in service of the ability of individuals and communities to interpret and predict the actions, dispositions, and thoughts of themselves and others, as well as to address their practical "person-related questions and concerns.,"285

Now that I have discussed the entrenched traditional Western view of selves and the alternative understanding of selves as narratives, the discussion turns to particular ways in which comics may implicitly reinforce the latter and undermine the former. Again, the formal features of comics implicitly present a narrative view of selfhood regardless of what selves actually are. And the fact that comics implicitly present a narrative view of selfhood can illuminate the cultural relationship between comics and subversive traditions, as I will explore in the next chapter.

\footnotetext{
${ }^{284}$ Schechtman, "The Narrative Self," 405.

${ }^{285}$ Schechtman, Staying Alive, 152.
} 
In the previous sections, I have examined the entrenched Western folk understanding of selfhood (influenced by the religious notion of the soul), which understands selves as unified and continuous essences. In contrast to the entrenched Western view, I detailed the assessment of some philosophers that selves are not unified essences, but rather "abstractums," or malleable interpretive narrative achievements. Below, I will again consider the elements of comics discussed in the previous chapter. Character depictions, artistic and design elements, and closure will all function to imply a view of the selves of comics characters as actively narrativized by readers. The examination of these three elements in relation to selfhood will be broad and overlapping, dealing with both the typical formal features and the content of various comics.

\section{Character Depiction and Selfhood}

As I explained in the previous chapter, depictions of characters can influence the emotional experiences of comics readers. Similarly, character depictions have the ability to influence readers' understandings of selfhood, both through their typical formal features and particular narrative content. A common way in which representations of characters in comics can influence readers' understandings and experiences of themselves is through the psychological mechanisms discussed in the previous chapter.

Specifically, readers may feel empathic, sympathetic, or antipathetic toward one or more of the characters depicted in a comics narrative. Such reader responses may cause a reader to reinterpret events in her past (such as, her prior thoughts and actions) or cause her to attempt to shape future actions based on the values that are communicated through a particular comic, or character in a comic, with which she comes to identify. 
This influence of the depictions of characters in comics relates to the narrative content, rather than only to the formal features of comics.

Brett Culp, life-long Batman fan and director of the documentary film Legends of the Knight, discussed his favorite character's influence on his own life and the lives of others:

Batman has been ever present in my life, for my whole life. At every stage of my life there was a Batman for me [...] so there has always been a Batman present for me at the level I was, and the Batman I needed at that time to be a hero for me $[\ldots]$ When real life heroes aren't there for us I think we tend to move more towards our fictional heroes, and that in my opinion speaks to the rise in the whole geek culture and our obsession with these stories of heroes in the fictional world because our real life heroes are not there for us the way they used to be. ${ }^{286}$

Batman, as we see, has influenced Culp's (and many others') values and understanding of himself and the world. Influence of this type over the understanding and experience that readers have of themselves is not unique to the medium of comics, even though the formal features of comics entail that the method of communication of such content is unique. However, beyond only influencing how readers think about aspects of themselves specifically, the comics medium also able to implicitly present a particular understanding of selves in general, as being narrative constructions.

One way in which the typical depictions of characters in comics may implicitly undermine the common Western conception of selves as unified essences is by depicting the same character multiple times on a single full-page frame. ${ }^{287}$ When a character is depicted engaging in a particular action or range of actions, it is not uncommon for a comics page to include several images of the same character at various points in the

${ }^{286}$ Brett Culp, "Interview: Brett Culp, director of the Batman Documentary Legends of the Knight," Flickering Myth, August 15, 2013.

https://www.flickeringmyth.com/2013/08/interview-brett-culp-director-of-batman.

${ }^{287}$ Eisner, Comics and Sequential Art, 65. 
action she is undertaking. As I discussed in detail in previous chapters, the formal features of comics entail that narrative time must be depicted using the space on a the pages of the comic. ${ }^{288}$

While comics readers (in the U.S. context) are likely to think that they themselves consist of unified essential selves, characters in typical comics narratives are not and cannot be depicted as such. Because shifts in narrative space and time in comics are actively imagined by the reader (that is, readers enact the imaginative element of closure), the reader must imagine the continuity and unity of comics characters, when characters are depicted in multiple varying images on a single page. The key point for the purposes of this discussion is that the reader does not perceive unity, continuity, or an essence of characters in a typical comics narrative, while the use of mimetic visual artwork foregrounds the fact that these features are lacking. This latter point is key because it distinguishes comics from other narrative media, such as prose novels. Consider the following example.

The highly influential creative team of Dennis O'Neil and Neal Adams authored the pivotal comic book issue Batman \#251 entitled "The Joker's Five-Way Revenge." This issue offers a narratively dark and murderous interpretation of the most popular Batman villain, the Joker. The story is simple: The Joker has escaped from custody and is attempting to murder the five members of his former gang because he believes one of them betrayed him, leading to his previous capture. When Batman discovers the Joker's intention, he attempts to protect the members of the gang before the Joker strikes. In the page below (Figure 19), Batman tries to talk the Joker's gang member named Bigger

${ }^{288}$ McCloud, Understanding Comics, 94-97. 
Melvin into placing himself in protective custody. Bigger Melvin seems open to

Batman's suggestion, but then attacks Batman when his back is turned.
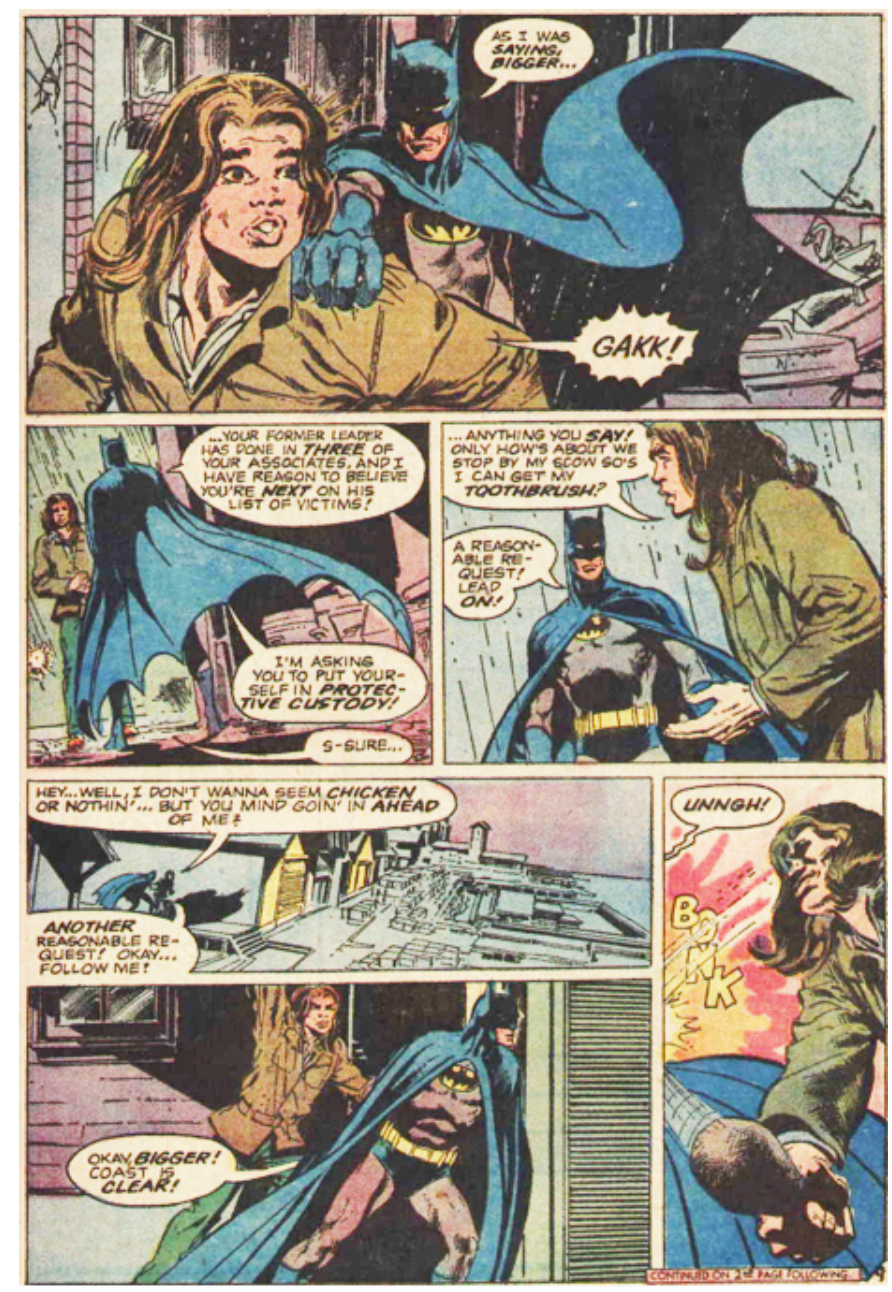

Figure 19: Denny O'Neil and Neal Adams, "The Joker's Five-Way Revenge," Stacked Deck: The Greatest Joker Stories Ever Told (New York: DC Comics, 1990), 173.

The reader who engages with “The Joker's Five-Way Revenge," necessarily does not visually perceive the continuity of the singular character of Melvin. In fact, Melvin is depicted six times on this page and in five of those panels he displays facial expressions that communicate a range of sometimes mutually exclusive emotional and psychological states. The first panel of the page displays Melvin as startled by Batman's hand on his shoulder. Responding in shock, Melvin exclaims, "GAKK!" In the second panel, Melvin's expression is less detailed but appears to display tentativeness and nervousness 
at being face-to-face with the vigilante. The third panel depicts Melvin as submissive and cooperative, as he agrees to Batman's request to take him into protective custody, as long as he can retrieve his toothbrush first. The fourth panel only includes a depiction of Melvin's bodily posture as he asks Batman to lead the way. In the fifth panel, the reader is able to infer that Melvin's previous displays of cooperation were a ruse as his face and bodily posture reveal his aggression toward Batman. In the sixth and final panel, Melvin is depicted as swinging a club and hitting Batman with a loud "BONK."

Here, the reader does not see the continuity of the character of Melvin across the gutters between any of the six panels. There also is no singular unified essence of Melvin that is depicted within each or any panel. There are similarities across the various depictions of Melvin that allow readers to infer and imagine (that is, enact closure) that a single character is depicted, but both his facial expressions and bodily postures shift from panel to panel, sometimes in ways that are mutually exclusive. For example, Melvin's calm submissiveness in the third panel is highly distinct from his startled expression in the first panel. Calm submission necessarily excludes startled tension. And while typical readers will actively enact closure in order to narrativize these distinct depictions into a conception of a single character, there is no unified, continuous, essential Melvin that tracks across the physical comics page. The reader must actively narrativize (through closure) the abstracted unification or continuity of Melvin.

In standard examples such as this, the reader enacts the process of closure, allowing her to infer and imagine various elements of the storyworld of the comic. The enactment of closure highlights (to those analyzing the medium and potentially to readers) that the selves of comics characters are actively projected, rather than being 
found within the physical images and textual elements of the comic. The uses of inference and imagination (that is, closure) allow readers to interpret and narrativize the visually perceptible physical depictions of comics characters as coherent selves that persist in the storyworld across the various depictions. So, the selves of comics characters are abstractums, actively interpreted by readers who enact the cognitive processes associated with closure.

I am not arguing that all or most readers will have difficulty reading comics characters as unified or subsisting across multiple panels. In fact, this should be a standard way of reading comics because of the enactment of closure. Rather, I am suggesting that the use of multiple varying images in standard character depictions foregrounds for readers the fact that they actively enact the understanding of characters as unified and continuous. The comics medium highlights the fact that the unity and continuity of characters are not simply presented by the physicality of the comic. We can easily distinguish between the physical depictions in the comic and the abstractum of the comics characters.

The fact that comics use mimetic representations of the characters and not only textual references potentially highlights the perceptual disunity of characters in the comic. This distinguishes the experience of reading a comic from that of engaging with other types of narrative media. For example, the reader of prose fiction also does not perceive the continuity, unity, or essence of characters. However, prose fiction only references characters and does not depict them. It is not that other types of narrative mediums $d o$ present readers with characters who are unified and essential, but rather than the formal features of comics have the potential to foreground the lack of unified 
essential selfhood and highlight the active projection by readers of unity and continuity onto depicted characters. This implicitly undermines the common Western understanding of selves.

Typical character depiction offers an example of what A. David Lewis describes, when he argues, "what may allow the graphic novel the ability to squeeze between readers' devotion to unified selfhood and the expectation of characters' unity is the medium's equal usage of pictures, its dual sign system of words and images."289 There is no depicted singular essence of Melvin in the example Figure 19. Rather, there are six instances of Melvin with similarities and differences that are actively narrativized into a continuous and singular Melvin in the minds of readers.

In this type of standard depiction of a character in comics, the reader holds multiple images of a character in her field of vision at once. While she may be focusing on a particular panel, other images of the same character occupy her visual field as well. As comics theorist Nick Sousanis argues, "while comics are read sequentially like text, the entire composition is also taken in - viewed - allatonce [sic]. ${ }^{, 290}$ Multiple character depictions viewed simultaneously implicitly suggest a disunified and potentially multiple selfhood for the depicted character and they reveal to the reader the actively narrativized nature of those characters. In other words, the reading experience of comics potentially makes the reader aware of the imaginative and inferential work that she must invest in order to understand each character as continuous and unified. The nature of the standard reading experience of comics, including closure, implies an actively narrativized

289 A. David Lewis, American Comics, Literary Theory, and Religion, 112.

${ }^{290}$ Nick Sousanis, Unflattening (Cambridge, MA: Harvard University Press, 2015): 62. 
understanding of selves and this implication undermines the traditional Western view of selves discussed in detail above.

The typical formal features of comics can also work in concert with the narrative content of a work to further emphasize a narrative understanding of selfhood. The specific genres in which characters are depicted can affect how selves are depicted and also how readers understand the meanings of the narratives in which those characters are portrayed. For example, comics theorist Rocco Versaci discusses the ways in which comics memoirs allow for possibilities of self-depiction that are unavailable to authors working with only prose. Versaci argues that comics constantly remind their readers of their constructed nature. ${ }^{291}$ Therefore, when memoirists utilize the medium to articulate an understanding of their self and their story, it:

reminds us at every turn that retelling one's personal history is, in part, an act of invention. That is, the very nature of the medium-the fact that the images are drawn, the details arranged within panels, the panels arranged within a pageforegrounds that the comic book is an active reconstruction of the past [or an active construction of a fictional storyworld]. While this foregrounding occurs with varying degrees of subtlety, its existence makes comics the ideal medium in which to explore how 'truth' is constructed, particularly in the memoir. ${ }^{292}$

The ways in which depictions of characters in comics engage readers' perceptual capacities (as well as their cognitive capacities) highlights an understanding of the selves presented in comics as actively narrativized. Memoirs have been a particularly popular genre in comics in the past several decades and relaying one's experiences in comics highlights the types of interpretive activities that constructions of self-narratives typically involve.

${ }^{291}$ Rocco Versaci, This Book Contains Graphic Language: Comics as Literature (New York: Continuum Intl. Publishing Group, 2007): 6.

292 Versaci, 58. 
It is worth briefly mentioning an interesting potential objection to my argument. Typically comics feature cartoon-style drawing, which involves highly contrasted outlines of objects and characters. ${ }^{293}$ So, cartooning often clearly delineates the boundaries of individuals and objects, differentiating them from their surrounding environment. One might argue that the common use of cartooning in comics would suggest that comics will often suggest a more unified or essentialist view of selfhood precisely because cartooning so clearly delineates characters from their surroundings (that is, cartoon drawings typically feature stark lines between the depictions of characters and the worlds those characters inhabit).

However, the sequential nature of images in comics, as well as innovative uses of artistic style and design elements (discussed in detail below), visually undermine the notion that there is a single stable entity represented by the boundaries around any particular character across a sequence of panels. While a single piece of visual artwork that featured a character drawn in a cartoon style might be read to imply a subsisting, unified, and essential self for that character, typical comics' use of sequences and the subsequent artistic and design possibilities entail that comics usually undermine such a reading of selfhood.

Whether engaging with depictions of characters in an art comic, graphic memoir, or mainstream superhero comic, readers are actively engaged (through closure) in projecting the unification, coherence, and narration of those characters. Similar to Dennett's notion of the self as a theorist's fiction, the self of a comics character is a narrative achievement resulting from active cognitive engagement and interpretation of

${ }^{293}$ Boyd, "On the Origin of Comics," 105. 
the reader. The narrativized self of a comics character is not depicted, perceived, or contained in the physical images or words of the comic. Readers actively interpret the selves of characters. Similarly, according to philosophers who hold a narrative view of the self, the selves of actual individuals are not contained in their physical existence or the history of their organism. Rather, the self is the abstractum that results from the active narrativizing and interpreting of that existence and history.

In the same way that our interpretations of others' or our own selves are constrained by certain facts about the life of the organism with which the self is associated, the interpretation of a character in comics is constrained by any clearly depicted or described information contained in the relevant comics. For example, I cannot interpret Melvin in Batman \#251 (Figure 19) as being a successful life-long pacifist because in interpreting the character I must take into account the information that the comic makes available about Melvin, including the depiction of his violent attack on the Batman.

Therefore, characters depicted in comics can also be conceived of as theorist's fictions. They are fictions in the relevant sense (regardless of the fictional/nonfictional status of the narrative as a whole) in that they are constructed and interpreted by readers when they enact the cognitive processes involved in closure. More specifically, the narrativized selves of characters depicted in comics are theorist's fictions because, if successful, they allow for understandings of those characters that best explain the images and words associated with the character and allow the reader to make reasonable predictions about the character's future behavior in the narrative. All of this follows from the typical formal features of and reader engagement with comics. 
Focusing on the content of particular stories, comics studies scholar A. David Lewis similarly argues that comics depicting single characters in wildly different ways (both visually and in terms of personality) may upset Western readers' assumptions about selves. He writes, "How is a reader who expects unified, whole characters to read passages that violate such assumptions?"294 While Lewis focuses chiefly on the content of superhero comics that depict the afterlife, as I have argued, the formal and representational features of comics alone can imply a view of selfhood as conventionally interpreted and narrative in nature.

As discussed above, characters in comics are presented to the reader as perceptually disunified because of the standard use of multiple depictions of an individual character on a full-page frame, which a reader is able to perceive and potentially attend to simultaneously. The unity of characters in comics is conventional because the understanding of characters as coherent and singular wholes relies on the conventions of comics reading communities and is not explicitly presented by the images of the comics themselves. And depictions of characters in comics suggest a narrative view of selfhood because the narrativizing of characters as singular unified wholes is not achieved by the content of the work itself, but rather readers must actively narrativize them. In short, the coherence of characters is not depicted in the comic.

However, the inclusion of static visual artistic elements within comics requires readers to engage with non-textual elements (such as, mimetic images, artistic style, page and panel design, etc.) when interpreting the narratives of characters. This distinguishes comics' potential to represent varying conceptions of selfhood from those available

${ }^{294}$ Lewis, American Comics, Literary Theory, and Religion, 110. 
through novels or poetry, which typically contain only textual elements. Readers of comics see the characters and the broader storyworld that they must interpret. Some perceptual information is provided by comics that is not provided by either novels or poetry, making the reading of comics characters a unique experience, and allowing comics to foreground a conception of selfhood as conventional and actively narrativized.

Visual Artistic and Design Elements and Selfhood

As I explained in the previous chapter, the artistic style and design elements of comics can shape the parameters for the potential emotional engagements of readers. However, these elements can also have implications for the views of selfhood that are communicated to comics readers. In the same ways as in visual artwork more broadly, the artistic style of visual art in comics may present a mood and general emotional tone. In cases where the artistic and design elements hold implications for the notion of selfhood, the representational content of the images is usually implicated, as well. In other words, artistic style and design elements are more likely to influence the understanding of selfhood communicated by the comic if the comic is about ideas like selfhood, personhood, identity, character, or other related notions.

For example, artistic and design elements can be used to explicitly depict characters as lacking essences or consisting of potentially disunified narrative interpretations. Consider the writer Alan Moore's influential run from 1984-1987 on DC Comics' The Saga of the Swamp Thing series. The Swamp Thing character was initially introduced in the 1970's as a scientist who had been caught in a laboratory explosion and transformed into a monster. Moore reconceived the Swamp Thing character, such that he consists of animated plant matter that has become conscious and mistakenly believes that 
it is the scientist named Alec Holland. Alec Holland, in Moore's story, died in the laboratory accident that created the Swamp Thing. But because Swamp Thing has many of the psychological features of Holland, the relationship between the Swamp Thing and the now-dead Holland is the stuff of philosophical thought experiments. There is continuity, but not identity, between Holland and the Swamp Thing. Swamp Thing's love interest Abby Arcane even comes to call him "Alec," though she is aware that he is not Holland but only animated plant matter.

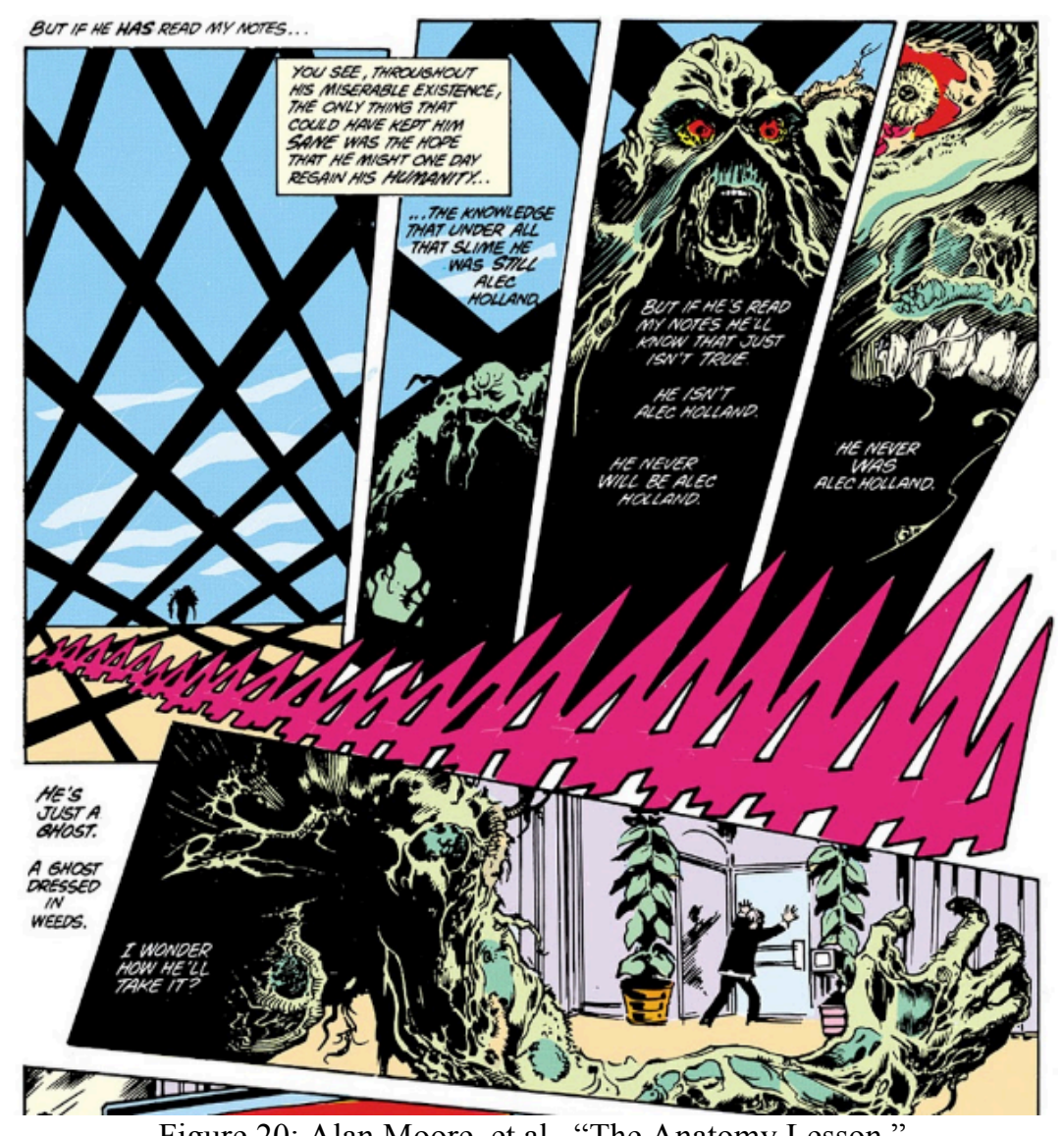

Figure 20: Alan Moore, et al., "The Anatomy Lesson,"

The Saga of the Swamp Thing Book One, \#21 (New York: Vertigo, 2012): 21.

The narrative, which deals with notions of identity, selfhood, and consciousness, allows for innovative medium-specific explorations of these concepts. In Figure 20 (above), we learn through the expositional text that the Swamp Thing is not Alec 
Holland, but his psychology is conditioned by Alec Holland's. While Swamp Thing has the memories and dispositions of Alec Holland, "he never was Alec Holland."295 The asymmetric and destabilized panel design visually communicates the disorienting experience of Swamp Thing's rapid change in self-understanding. Each panel in the sequence depicts him from an increasingly close perspective, which highlights the detailed strands of plant matter that constitute his body. This sequence of visual art communicates through stylistic and design elements that there is no ultimate unity, no essence of Alec Holland, "under all that slime",296 that makes up the Swamp Thing. Additionally, artistic and design elements may be used to depict characters in ways that undermine the typical division between a character and the rest of the storyworld (that is, between a depicted self and her depicted environment). In The Saga of the Swamp Thing, the titular character has the ability to grow potato-like tubers at will using the plant matter that constitutes his body. In the images below (Figures 21-23), we see the effects on the character of Abby when she eats one of the tubers. Readers are shown Abby's experience through psychological hermeneutic images (that is, images that visually represent the character's subjective experience). Abby's psychedelic experience undermines the division between her self and her environment. Her shift in consciousness and self-conception is communicated using the non-textual elements of the comic.

\footnotetext{
295 Moore, et al., "The Anatomy Lesson," 21.

${ }^{296}$ Moore, et al., 21.
} 


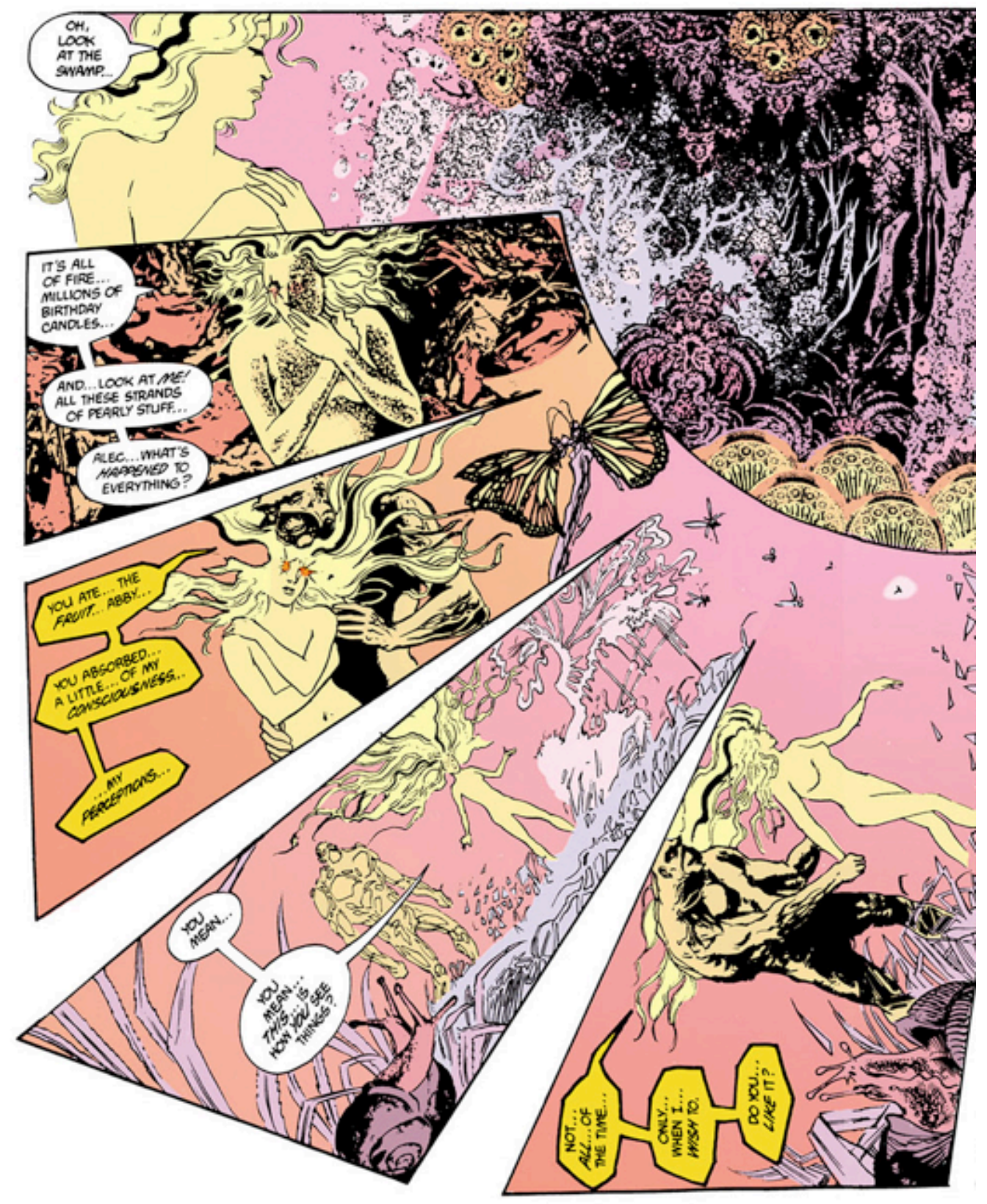

Figure 21: Alan Moore, et al.,"Rite of Spring," The Saga of the Swamp Thing Book Two, \#34 (New York: Vertigo, 2012): 11.

In the figure above (Figure 21), the structure and placement of panels is used to highlight the shifting of Abby's experiences from moment to moment. In order to read the entire sequence on this page (as well as the following pages), readers must turn the comic book on its side. The shift in Abby's conscious experience is accompanied by a 
shift in the orientation of the artistic representations of that experience. Also, the unconventional panel structure suggests an ambiguity in the communication of narrative time. Specifically, the curved fan-like panel structure that requires the turning of the comic book does not only serve to shift the orientation of the artwork. But also what would typically be multiple panels (and thus, multiple narrative moments) are connected along a curved line on the right side, with butterflies and other insects depicted as flying across the implicit divides between panels. The lack of gutters and lined divisions between panels further visually communicates the dissolution of boundaries that Abby begins to experience as her consciousness shifts. 


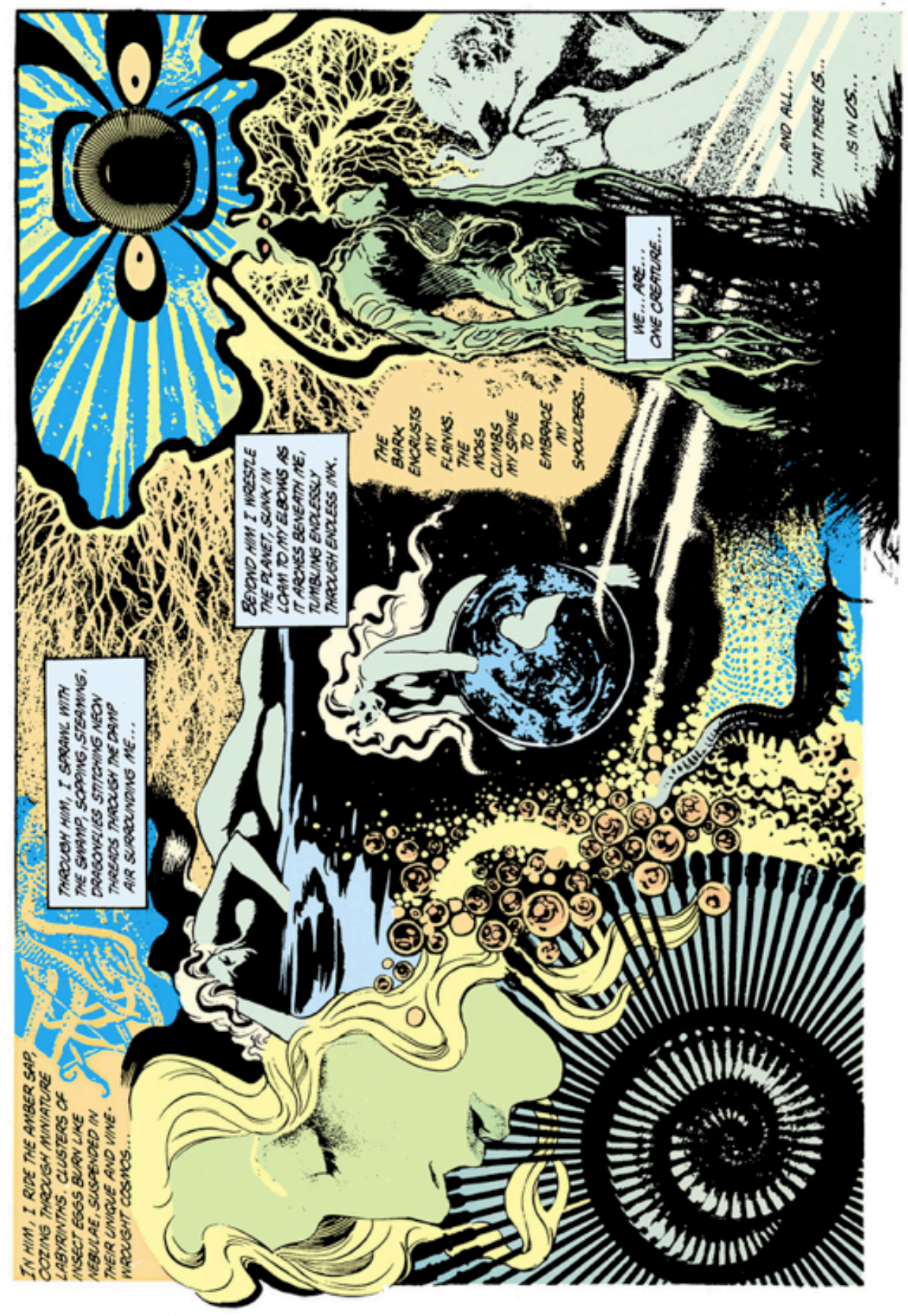

Figure 22: Moore, et al.,"Rite of Spring,” 13.

As the effects of the tuber on Abby's consciousness continue, the typical

conventions of the comics medium are subverted further. The full-page frame above

(Figure 22) does not clearly communicate narrative time or location. There are no gutters or clear panel distinctions and while the reading order of the textual elements leads the eye from the top left to the bottom right, it doesn't seem that narrative time is being 
depicted as progressing in that direction on the page. In fact, the textual elements on the page suggest a timeless nature to the depicted experience, as it is described using only present-tense language. The experience is described as "tumbling endlessly, in endless ink. ${ }^{, 297}$

${ }^{297}$ Moore, et al.,"Rite of Spring," 13. 

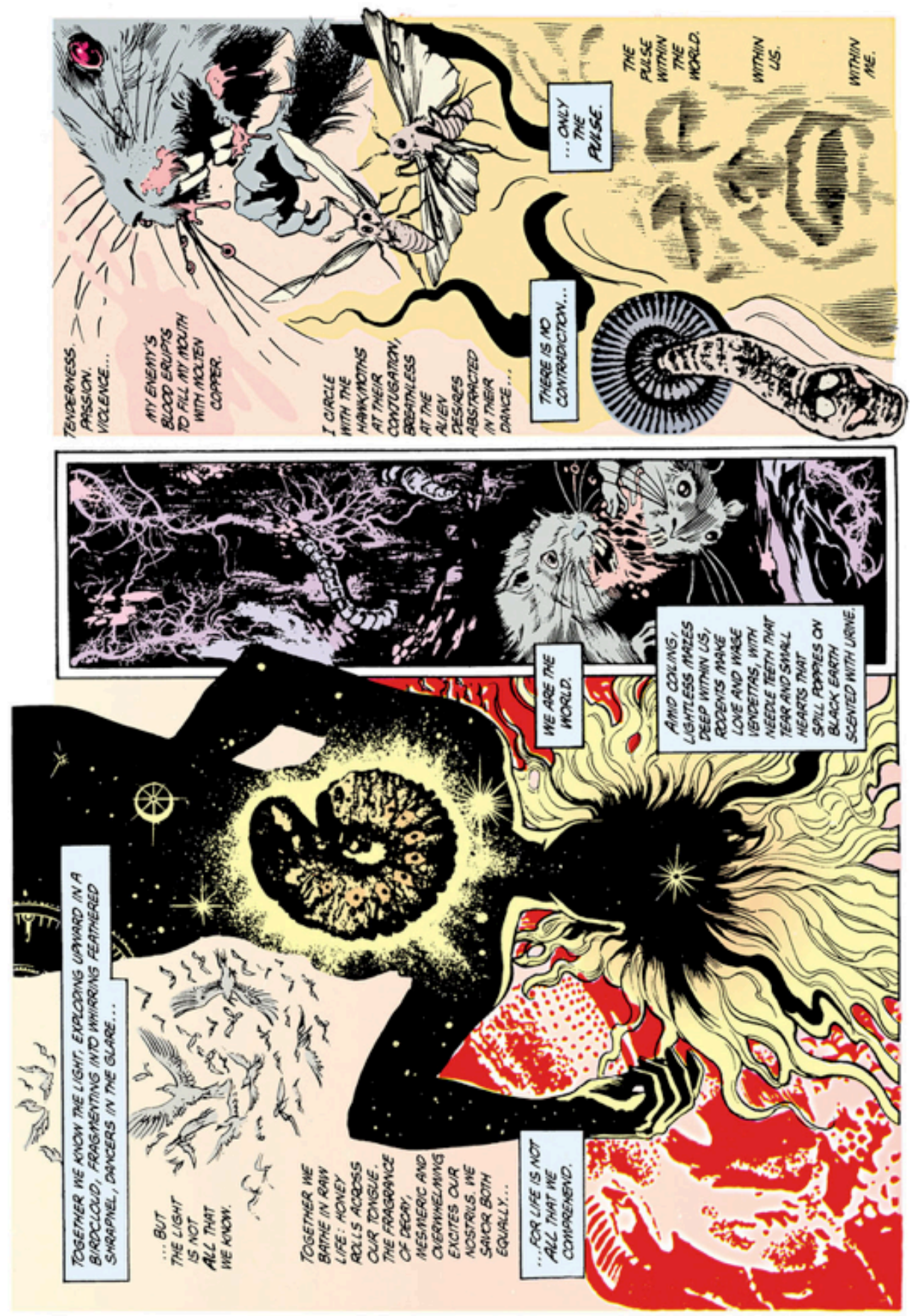

Figure 23: Moore, et al.,"Rite of Spring,” 14.

In Figure 23 (above), the artwork continues to depict Abby's sense of selfhood as dissolving into her environment. While this full-page frame includes a version of a typical panel structure with a bordered panel in the center, the page must be read with the comic on its side like the previous pages. This communicates the disorientation of the 
character's experience, as well as replicating it for the reader to a degree. Additionally, the silhouette of Abby is used as a border for images of a starry sky and some type of larval insect creature. This suggests the emptiness or indistinctness of her identity from the rest of her world during this experience. The dissolution and disruption of Abby's identity is expressed through the textual elements of the page also. Abby begins to identify herself not only with the Swamp Thing by using the term "we," but also with all things, by saying "we are the world." 298 In other words, the experience communicated to readers is not simply one of general disorientation, but of disorientation revolving around an upset in the traditional sense of selfhood as singular, unified, and essential. The boundaries between self and non-self are malleable.

These examples in The Saga of the Swamp Thing go farther than simply offering a distinct conception of selfhood, in that they attempt to depict and describe an experience of the loss of a standard sense of self. These types of experiences are typically associated with mystical religious, esoteric, and occultist traditions, as well as the use of psychoactive substances (such as the tubers grown by the Swamp Thing). It is this link between the conceptualization of the self as a narrative construction and the potential experience of the suspension of the self-narrative that illuminate the relationships between the formal features of comics and various esoteric and occultist ideas in the U.S. over the past century. The next chapter will explore examples of this cultural relationship in detail and highlight how these implications of the formal features of comics play out in terms of narrative content and cultural meaning.

${ }^{298}$ Moore, et al.,"Rite of Spring,” 14. 
The previous examples highlight the role that artistic style and design elements can take in offering a vision of selfhood as inessential, narrativized, and potentially disunified. As comics theorist Nick Sousanis writes:

When we take the all-over composition into account, form and expression become one. Meaning is thus conveyed not only by what's depicted, but through structure: the size, shape, placement, and relationship of components - what they're next to and what they're not, matters. [...] Through [comics'] multiplicity of approaches for constituting experience, this form can provide an elevated perspective from which to illuminate the traps of our own making and offer a means to... step out. $^{299}$

With regard to these pages from Alan Moore's run on the Swamp Thing series, Sousanis' insight allows us to see that comics can offer readers the opportunity to step outside of their typical understandings of selfhood, at least conceptually. Comics can offer this interpretive possibility through the textual elements, artistic styles, page layout, and panel design.

Examples that involve longer narratives including characters with whom readers may identify (like those discussed above) are more likely to communicate notions of selfhood in an emotionally impactful way. However, instances of the comics medium that explore unique representations of selfhood can be seen in less narrative examples as well. For example, the comics artist John Cullen (creating under the pseudonym of his name in reverse: Nhoj) creates daily short online comics, many of which offer innovative uses of the formal possibilities of comics. The example below, aptly entitled "nonlinear," does not include an obvious narrative sequence.

${ }^{299}$ Sousanis, Unflattening, 66. 


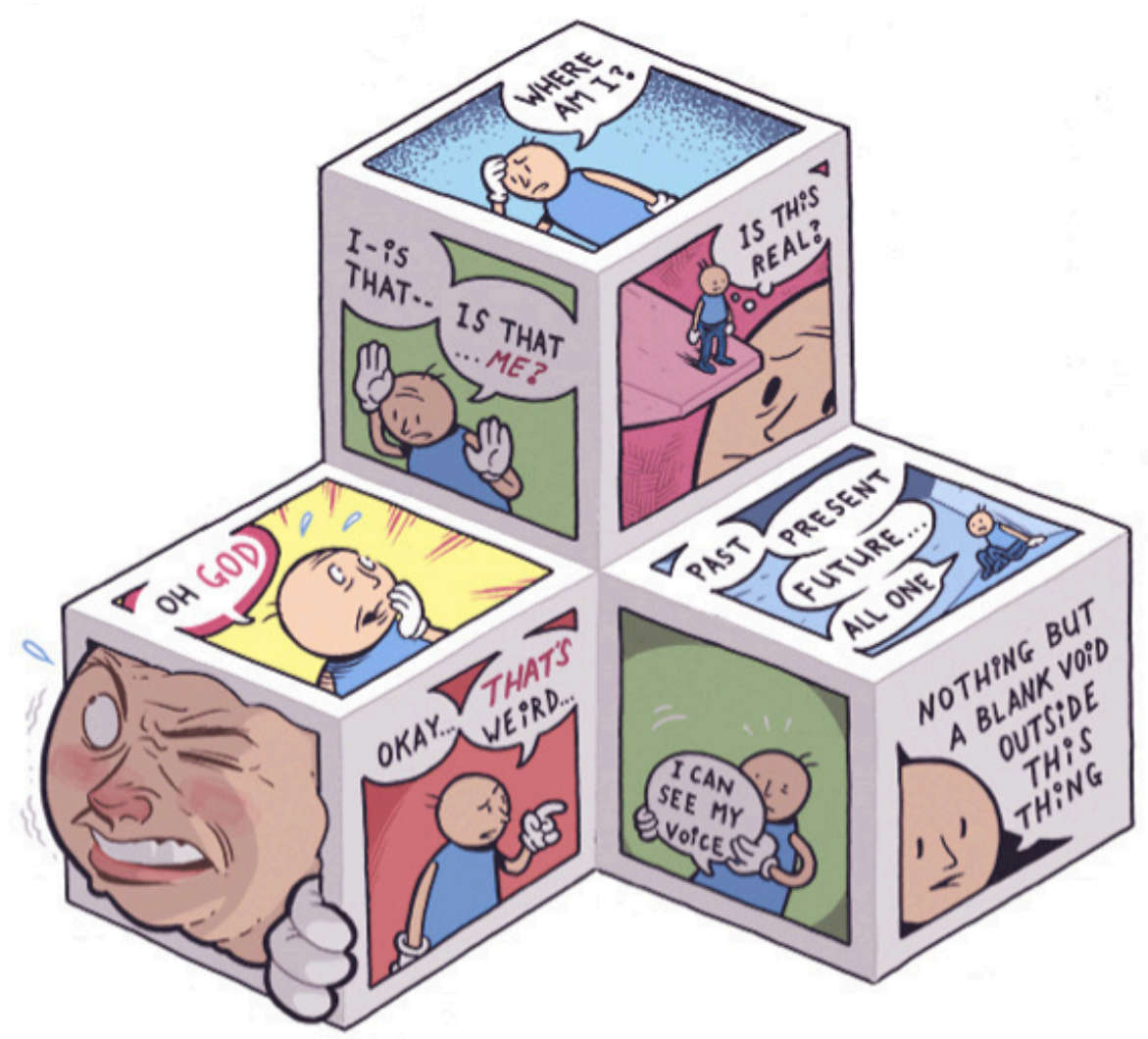

Figure 24: John Cullen, "nonlinear," Nhoj, September 8, 2016, http://nellucnhoj.com/post/150125629359/nonlinear-tumblr-twitter-facebook.

The different sides of the depicted three-dimensional object take the place of standard panels. This functions to negate the typical use of panels as successive moments in narrative time. Rather, all panels exist simultaneously and although the content of certain panels only makes sense in relationship with certain others, there is no clear sequence of engagement that is necessary in order for the reader to understand the relationships between any of the panels. So in a way similar to the depiction of Abby Arcane's experience of the renegotiation of the conventional boundaries of selfhood, this example also features (albeit in a distinct way) the lack of typical narrative time.

Also, issues of selfhood are brought to the fore in "nonlinear." Through the textual and imagistic content of various panels (or sides of the depicted structure) the comic contains ten depictions of the same character. The character looks very similar in 
each depiction and in one panel says "I-is that-- is that ...me?" ${ }^{300}$ Not only does the character appear multiple times in the reader's visual field, but the character is apparently aware of the typically non-diegetic elements of the comic, such as the content of other panels, blank space outside the panels, and speech balloons.

In "nonlinear," the reader is presented with a character reckoning with the implications of existing in comics form. The character experiences disorientation, shock, and confusion around notions of time, place, and identity. He questions his location, as well as the reality of what he experiences, and even unsuccessfully attempts to escape from the panel on the bottom left. "Nonlinear," explicitly explores issues related to the disunity, conventionality, and actively narrativized understanding of the selves of comics characters by utilizing the potential of comics' formal features in innovative ways.

This example showcases the implications for selfhood provided by the design elements of comics even in lieu of a fully narrative structure. As we have seen, typical and a-typical depictions of characters, artistic style, and design elements have implications for the conceptions of selfhood on offer within comics. Namely, these aspects of the medium implicitly (and sometimes explicitly) suggest a reading of selfhood as narrativized and conventional, undermining the traditional Western view of selves as unified and continuous essences.

Closure and Selfhood

Again, the discussion returns to the paradigmatic type of engagement that readers of comics enact: closure. As I discussed above, depictions of singular characters in typical cases of comics narratives implicitly undermine the entrenched Western reading

${ }^{300}$ Cullen, "nonlinear." 
of selfhood by depicting characters multiple times on a single page. However, typical cases of the enactment of closure in comics are not the only ways in which the need for closure can influence the representations of selfhood in comics.

Some comics utilize the need for readers to enact closure in an particular way in order to illuminate the meaning of the narrative. Consider the crime/superhero trade paperback Powers: Forever, written by Brian Michael Bendis and penciled by Michael Avon Oeming. Powers: Forever focuses on the lifespan of a superpowered and nearly immortal individual named "Walker," as he struggles to remember and rationalize his distant past. At one point, Walker meets with Albert Einstein, thinking that the genius physicist will be able to help him make sense of his immortality.

Einstein, rather than explaining Walker's condition, offers him a lesson on the nature of time. He claims, "Man created time. Time is a fiction. Do you understand? [...] A human being is a part of the whole called by us a 'Universe' [...] He experiences himself, his thought and feelings as something separated from the rest: a kind of optical delusion of his consciousness." ${ }^{301}$ These views of humans being ultimately indistinct from the whole of the Universe and time being an illusion are enhanced and supported in the readers' experience through the mimetic representations that comics afford. Einstein's face is depicted in multiple disjointed panels across what is apparently a very short span of narrative time. This design choice serves to highlight and undermine the conventional nature of the passage of narrative time and of the unity of Einstein as a character and of all characters, as he is discussing the nature of humans in general.

${ }^{301}$ Brian Michael Bendis and Avon Michael Oeming, Powers: Forever (New York: Icon Comics, 2004): 115. 


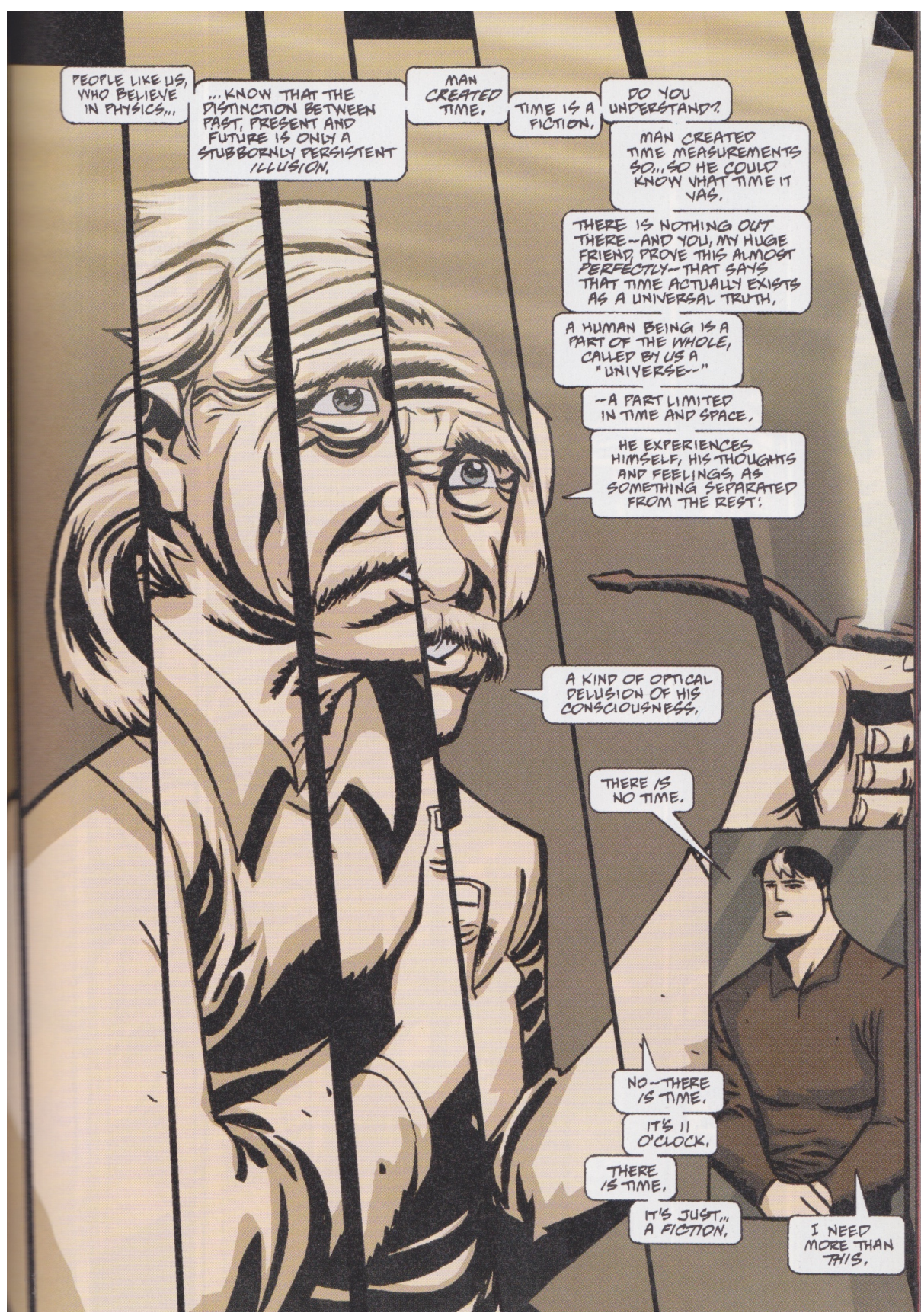

Figure 25: Brian Michael Bendis and Avon Michael Oeming, Powers: Forever (New York: Icon Comics, 2004): 115.

The above image (Figure 25) exemplifies the ability of comics to subvert the view that selves (in this case, the character of Einstein) are continuous or intrinsically unified essences. Here we see an example of comics' formal and representational features and the necessity of closure in reader engagement implying that the characters within comics can 
be rightly thought of as narratively constructed by the reader. Examples like the one above provide readers with the opportunity to apply this notion of selfhood to selves in the actual world, as well. In other words, readers of comics are presented with opportunities to understand themselves as potentially disunified, conventional, shifting, and narrativized.

Potential Upsets in Readers' Experiences of Self

There are many ways in which a narrative may affect readers' conception of selves in general or of themselves in particular. Probably the most common way in which narratives can influence one's conception of themselves is in regard to character or personality traits. For example, a reader may engage with a narrative in which a particularly brave character is venerated, either explicitly by other characters or a narrator within the story or through criterial prefocusing by shaping reader attention in such a way as to lead her to see the brave character as admirable. The reader may then compare her own personality to that of the brave character. This may lead the reader to wish that she were braver or to attempt to bolster her own bravery in certain situations as a result of admiring the brave character. ${ }^{302}$

${ }^{302}$ Though it is not the focus of the current exploration, it is important to acknowledge the social dynamics of narrativized selves, as well. Identity categories that are given salience by their social contexts and histories also are relevant to how particular comics narratives may aid readers in the understanding or reimagining of themselves. For example, comics narratives that represent gender, racial, ethnic, or religious identities are written and read by people who are imbedded in normative cultural systems. The use of emotional salience and narrative tropes in a comic can serve to strengthen or undermine broader cultural values. Batman, for example, is portrayed as a hero and protagonist who is mostly venerated throughout his long history in comics. However, Batman also is portrayed as a straight white wealthy man, which undoubtedly has implications for various readers' interactions with the character depending on how their own social positioning influences their sense of self. 
Additionally, narratives may thematically explore issues of selfhood more generally. Superhero stories, which "established the comic book as a commercially viable medium in the United States, ${ }^{303}$ typically utilize certain narrative tropes that have implications for readers' understandings and experiences of selfhood. Specifically, the "dual identity" ${ }^{304}$ aspect of superhero narratives showcases a constructed and social understanding of selfhood and identity, which I will discuss further in the next chapter. The dual identity aspect of the narrative content of many superhero comics emphasizes the function that social roles fulfill in our understandings of the particulars of our selfconcepts.

The medium that a story is communicated through, rather than only the narrative itself, influences the types of representations of selfhood on offer. As I have argued, comics, by dint of their formal features alone, implicitly offer readers a conception of selfhood that undermines the entrenched Western view. A. David Lewis discusses the textual and visual elements of comics as being "like an $x$-and- $y$ coordinate system" ${ }^{\text {"305 }}$ that co-define readers' understandings of characters. And "with so much of a comic taking place within the reader's mind [they] may be loosed from conceiving characters in [a] confined manner $[\ldots]$ and compelled to regard them in less familiar, potentially more transcendent ways. ${ }^{306}$ However, these atypical conceptions are not necessarily confined only to readers' understandings of the characters presented in comics. Readers may apply these readings of selfhood to their own self-understandings as well, potentially providing them with similar conceptions and experiences of themselves.

\footnotetext{
${ }^{303}$ Duncan and Smith, The Power of Comics, 242.

${ }^{304}$ Duncan and Smith, 222.

${ }^{305}$ Lewis, American Comics, Literary Theory, and Religion, 132.

${ }^{306}$ Lewis, 133.
} 
The potential for anomalous experiences of selfhood have been reported in many cultures globally for at least several millennia. These experiences are often associated with religious practices and/or various types of intoxicants. Mystical practitioners of various religious traditions have written in numerous ways about experiencing the loss of a conventional sense of unitary or essential selfhood. Such self-loss experiences are continuous with the mainstream doctrines and practices of some religious systems (e.g., Buddhism, Hinduism), while other religious traditions are typically less amenable to practitioners' descriptions of such experiences and the conceptual understandings of selfhood that often accompany them (e.g., Christianity, Islam).

For example, many streams of Buddhism not only recognize of the self as conventional but also idealize experiences of the loss of the conventional self. ${ }^{307}$ The Zen Buddhist teacher Shunryu Suzuki wrote, 'If you think, 'I breathe,' the 'I' is extra. There is no you to say 'I' [...] 'You' means to be aware of the universe in the form of you, and 'I' means to be aware of it in the form of I." ${ }^{\text {308 }}$ Mainstream versions of more theistically focused traditions, such as the Abrahamic monotheisms, have tended to relegate such experiences to their more mystical manifestations and sometimes talk of such experiences have been denounced and considered heretical. ${ }^{309}$

\footnotetext{
${ }^{307}$ Mitchell, Buddhism.

${ }^{308}$ Shunryu Suzuki, Zen Mind, Beginner's Mind: Informal Talks on Zen Meditation and Practice, ed. Trudy Dixon (New York: Weatherhill, 2002): 29.

${ }^{309}$ The World Religions: Western Traditions (Fourth Edition), eds. Willard G. Oxtoby, Amir Hussain, and Roy C. Amore (New York: Oxford University Press, 2014): 247-48; Raymond Blakney, trans., Meister Eckhart: A Modern Translation (New York: Harper \& Row, 1941): xx-xxi. For instance, the Islamic Sufi Mystic Husayn ibn Mansur al-Hallaj was executed in the tenth century for the heresy of denying the distinction between his identity and the identity of God. Similarly, the fourteenth century Christian teacher Meister Eckhart was posthumously excommunicated from the Western Roman Catholic church on grounds of mystical heresy. Eckhart's teachings primarily focused on "the
} 
I have written elsewhere about how the experience of loss of the conventional day-to-day experience of self can be understood within the context of the philosophical view of the self as narrative in nature. ${ }^{310}$ Self-loss experiences involve the renegotiation of the typically perceived boundaries of the self, which is fully explicable by understanding the self as an "abstractum" or "theorist's fiction."311 That is, if selves are narrative in nature and are a product of interpretation, then the boundaries of a given self may be renegotiated conceptually and experientially, within certain limits. It is this possibility that many comics foreground through their formal features.

In comics, "time and space unfold optionally, either forward and in sequence as one might commonly expect or in more elaborate, transcendent configurations." ${ }^{312}$ So, because comics offer readers potentially unconventional representations of the selves of characters and narrative temporality, they may also offer readers a potent tool to understand or investigate experiences of the suspension of their own conventional selfhood.

Some authors have recognized this potential of the comics medium and have attempted to influence their readers' understandings of selfhood explicitly. For example,

unity of the divine and the human [and the notion that] if God and man are of the same genus, it must be possible to set free the divine kernel of being in man's inmost self by the ever-increasing conquest of his outer self-identity." Eckhart's excommunication and al-Hallaj's execution both speak to the potentially subversive nature of renegotiations of the boundaries of selfhood within certain theistic religious systems. Most relevant for our purposes is that religious systems that take this view of selfhood were and are highly influential on the cultural landscape of the U.S. in the Twentieth and early Twenty-first centuries, when comics matured and gained popularity as a medium.

${ }^{310}$ Will Simpson, "The Mystical Stance: The Experience of Self-Loss and Daniel Dennett's 'Center of Narrative Gravity,"' Zygon: Journal of Religion and Science 49, no. 2 (2014): 458-475.

${ }^{311}$ Dennett, "The Self as a Center of Narrative Gravity," http://cogprints.org/266/1/selfctr.htm.

${ }^{312}$ Lewis, American Comics, Literary Theory, and Religion, 67. 
Grant Morrison's series The Invisibles, published from 1994-2000 and later collected into seven trade paperbacks, offers a disjointed and complex narrative following the titular group of magical "freedom fighters" ${ }^{313}$ as they confront various threats to human spiritual (and social) freedom. Attempting to offer a succinct summary of the plot points contained in Morrison's creator-owned series is "a losing proposition." ${ }^{314}$ In part, this is because the obscure and dense mythological narrative contains references to "everything from the Marquis de Sade to H.P. Lovecraft, William S. Burroughs to Philip K. Dick, esoteric Christianity to Zoroastrianism. It's the closest you can get to spending a few days lost in Morrison's head, where Morrissey lyrics bump up against Terence McKenna's 2012 theories. The resulting disorientation is part of the book's appeal." ${ }^{315}$ And the series is very disorienting.

However, one of the clear themes of the narrative is the undermining of the binary between the forces of good and those of evil. By the end of the series, Morrison blurs the clear lines of division between The Invisibles and the Outer Church (the antagonists of the series), with the two groups apparently working toward a common apocalyptic purpose unbeknownst to their respective members.

This theme of gradual unification functions not only to the dissolve the narrative boundaries between the protagonists and antagonists, but also to undermine "the

\footnotetext{
${ }^{313}$ Megan Goodwin, "Conversion to Narrative: Magic as Religious Language in Grant Morrison's Invisibles," in Graven Images, eds. A. David Lewis and Christine Hoff Kraemer (New York: Continuum Intl. Publishing Group, 2010): 259.

${ }^{314}$ Jesse Hicks, "The Classics: 'The Invisibles,"” The Verge, December 22, 2012, https://www.theverge.com/2012/12/22/3793690/the-classics-grantmorrison-the-invisibles.

${ }^{315}$ Hicks, https://www.theverge.com/2012/12/22/3793690/the-classics-grant-morrisonthe-invisibles.
} 
boundary between self and not-self., ${ }^{316}$ One of Morrison's characters articulates the loss of a conventional sense of self as a part of the eschatological climax toward which the series has been driving, saying that individuals will gain "access to multiple self-images and potentials, a menu selection of faces, contradictory personas, the end of notions of territory and boundary $[\ldots]$ The very concept of the individual, like that of the bounded nation-state was not designed to survive the last millennium and must be transcended." 317 In other words, part of the apocalyptic climax of The Invisibles is the realization that individual selves are simply conceptual interpreted constructions.

Similar to Moore's Swamp Thing run, The Invisibles utilizes the formal features of the comics medium in order to depict the interrelation between selves, narratives, and the use of language. In the panels below, which come from the final issue of the series, The Invisibles' member known as "Ragged Robin" reflects on the relationship between her selfhood and the narrative of that selfhood. In one panel she says, "the thing that scares me most; if I finish the story what happens to $m e$ ? Does it go on without me? Or is it the other way around?"318 In the following panel, she is seen floating among various words and phrases. Her location is unspecified and her own language seems to break down, consisting of mostly rough phonetic approximations of words. This disorientation regarding the character's self and that self's relation to language is influenced by Morrison's personal esoteric spiritual practices. ${ }^{319}$

\footnotetext{
${ }^{316}$ Goodwin, "Conversion to Narrative," 266.

${ }^{317}$ Grant Morrison, The Invisibles: The Invisible Kingdom (New York: DC Comics, 2002): 270.

${ }^{318}$ Morrison, 275.

319 "Magic Works: An Interview with Grand Morrison Part 1," Mondo2000, February 2017, http://www.mondo2000.com/2017/08/14/magic-works-an-interview-with-grantmorrision.
} 




Figure 26: Morrison, The Invisibles: The Invisible Kingdom, 275.

Comics, given the ways in which readers interact with them and their implications for notions of selfhood, are a likely medium in which to find narratives featuring esoteric, occultist, and potentially subversive ideas regarding the conception of selves. Through the use of images, comics can "streamline the process by which readers accept options that seem to violate bedrock reality." ${ }^{, 320}$ Among these conceptual options is the notion that the deep-seated Western understanding of selves as essential and unified is mistaken. Rather, comics imply that selves are abstracted narratives, constructed actively over time by the interpretations of individuals and communities.

${ }^{320}$ A. David Lewis, American Comics, Literary Theory, and Religion, 109. 


\section{Conclusion}

In this chapter, I have made the case that the traditional Western folk understanding of selfhood is that selves are unified and continuous essences of people and that this understanding is based on various cultural factors, including the religious conception of the soul. The cultural dominance of the religious understanding of humans as consisting of souls makes other understandings of selves potentially culturally subversive, even beyond any direct critiques of religious doctrines. Therefore, this view of selves as unified essences is not only common but also culturally normative.

One alternative understanding of selfhood is the philosophical view that selves are abstract narratives, constructed by individuals and communities. Versions of this view, such as Dennett's center-of-narrative-gravity view and Schechtman's narrative selfconstitution view, frame selves as conventional constructions that result from humans' tendency to narrativize certain physical events (namely, those involving their own organisms and the organisms of others). Out of this interpretive narrativizing, the stories of selves are constructed by individuals in conjunction with others' understandings and the views about reality that are affirmed within their specific cultural contexts. The view that selves consist of narratives can therefore be potentially subversive in contexts where the view that selves are unified, subsisting, and essential is considered normative or virtuous.

As I have argued here, the formal features of comics imply a narrative reading of the selves of characters depicted. The use of mimetic visual artwork in comics, along with the fact that typical comics pages contain multiple depictions of any single character, entail that no unified or continuous self of a comics character is presented by 
the pages of the comic itself. Rather, readers of comics must imagine and infer (that is, enact closure) that certain disparate images depict the same character over shifts in narrative time and space. Beyond only the typical depictions of characters, visual and artistic design elements that present non-standard representations of characters may also provide readers with opportunities to view selfhood as narrative and conventional (such as, Alan Moore's The Saga of the Swamp Thing). In such cases, the narrative content of a comic may also engage with issues of selfhood and personhood.

The fact that comics imply such an understanding of selfhood also offers readers the opportunity to reflect on their understanding of selves in general, rather than only the selves of the characters depicted in the comic. Therefore, readers may come to consider their own selfhood in conventional, narrative, and thus potentially subversive terms. That is, comics' implications regarding selfhood may help to explain the comics medium's cultural relationship to and tendency to represent non-normative and subversive systems of thought and ideas. In the following chapter, I will consider the cultural implications of the conclusions I have reached so far. I will argue that there is substantial culturalhistorical overlap between the comics medium in the U.S. and esoteric and occultist traditions.

Esoteric and occultist symbolism and fantastical stories and ideas have been staples of comics narratives since comic books' rise to popularity in the 1930's. I will examine these cultural connections and argue that the view of selfhood as narrative, interpreted, and conventional, which we have seen is implied by the very form of comics, resonates with many of the themes and ideas that appear in the content of comic books in the Twentieth and Twenty-first centuries. Thus, we will see that much of the fantastical, 
mythical, and religiously resonant themes that are commonly associated with comics narratives are not only an accident of cultural history, but are related to the ways in which the comics medium communicates content and the ways in which readers engage with that content. The stories that comics often tell are influenced by the ways in which we read comics. 


\section{IV: OCCULTISM, ESOTERICISM, AND THE COMICS FORM}

The Illusory Nature of Selfhood and Traditions of Occultism and Esoterica

Thus far, this work has taken a primarily philosophical and formalist approach to certain features that are paradigmatic of most comics in the U.S. context. By examining formal features, including panels, gutters, visual art, and design, I have elucidated readers' perceptual, cognitive, and emotional experiences with comics and explored the implications of those experiences for the concept of selfhood. At this point, I take the majority of my philosophical work to have been completed. In this final chapter, I will explore some of the ways in which the previously examined elements of comics have influenced the types of stories that many comics contain. The ways in which comics present narratives to readers, as examined in the previous three chapters, has a clear influence on the types of stories with which comics are popularly associated.

Readers engage with comics perceptually, cognitively, and emotionally in ways that have the potential to imply a narrative and destabilized conception of selfhood. As I explained in the previous chapter, the fact that this view is potentially subversive in the Western cultural context will help to illuminate the tendency of comics to present culturally subversive themes with regard to conceptions of identity and selfhood. The comics medium, and not only particular narratives in comics, has faced criticism for being subversive in various ways over the course of its popularity throughout the past 
century. The history of comics in the U.S. also involves many points of contact with, and many comics contain representations of, esoteric and occult traditions.

A brief qualification on definitions is necessary before I move forward. By "esoteric" and "occult," I mean those traditions and movements that are often religiously resonant and that involve hidden beliefs and practices, as well as those that incorporate gnostic features. Scholar of esotericism, Arthur Versluis writes, "gnosis refers to a direct insight into what is largely hidden from rationalistic modes of knowledge." ${ }^{321}$ Esoteric and occultist traditions are often culturally or religiously subversive. They tend to focus heavily on the experiences that are available to practitioners or followers, more than focusing on which beliefs or practices are considered to be approved and proper.

Beyond that, occult and esoteric traditions often seek to undermine to or dissolve the boundary between the self of the practitioner and the rest of the universe (or larger reality, however it is conceived). ${ }^{322}$ Versluis writes:

Western esoteric traditions - and this is true of all of them - are founded on the existence of $[. .$.$] the divine - that transcends and links both humanity and the$ cosmos; and Western esoteric literature, be it Rosicrucian or alchemical, theosophic or Kabbalistic or magical, only works by reference to this third element, the divine, whose fundamental nature is to join together self and other. ${ }^{323}$

${ }^{321}$ Arthur Versluis, Magic and Mysticism: An Introduction to Western Esotericism, (Lanham, MD: Rowman \& Littlefield Publishers, 2007): 4. By "esoteric" and "occultist," I mean those traditions and movements that are often religiously resonant that involve hidden beliefs and practices, as well as those that incorporate gnostic features. Esoteric and occultist traditions are often culturally or theologically subversive and tend to focus heavily on the experiences that are available to practitioners or followers.

${ }^{322}$ Arthur Versluis, "Western Esotericism and Consciousness," Journal of Consciousness Studies 7, no. 6 (2000): 29. Even in cases when esoteric and occultist traditions utilize the term "soul," they generally still maintain the possibility (and desiribility) of experiences of the dissolution of the boundaries of between the individual and the higher reality (Divine/God/Reality/Cosmos/etc.). The use of the term "soul" in these cases bears little on the arguments made here, as the esoteric views about "souls" distinguish them clearly from the folk notion of a unified continuous essential self.

${ }^{323}$ Versluis, 23. 
The claim of this chapter, then, is that the tendency of many comics to portray and engage with such subversive religious and spiritual traditions is not simply an historical accident, but is made particularly likely in light of the ways in which people read comics generally. Comics are particularly well placed to represent and communicate ideas that are convergent with esoteric and occult traditions, which similarly question the common Western understanding of the self. In the previous chapters I have detailed the common types of reader engagements with comics and going forward I will elucidate the ways in which their cultural history exemplifies and manifests the philosophical points made thus far. In short, I will show that esoteric and occultist thematic elements are likely to appear (and have historically appeared) in comics stories, and that this likelihood is intimately related to the particular ways in which comics use image and text in conjunction with one another, thus undermining the common Western notion of an essential self.

A brief caveat is in order. I am not claiming that narrative themes in comics that parallel occultist and esoteric notions are intentional in all or most cases. It is certainly not the case that all (or even most) comics authors or artists over the past century have been occultists, adhered to esoteric worldviews, or explicitly sought to promote such views. Though some have and do. However, as I will show, many of the narrative genres most popularly associated with comics commonly include stories that undermine the common Western notion of the self in ways that converge with occult and esoteric traditions of thought. These comics exemplify the philosophical claims of the previous three chapters. ${ }^{324}$

\footnotetext{
${ }^{324}$ Nelson Goodman and Catherine Z. Elgin, Reconceptions in Philosophy and Other Arts and Sciences (Indianapolis: Hackett Publishing Company, 1988): 21, 40-41. These
} 
Given the tendency of comics to portray occultist and esoteric narrative themes, it is no surprise that comics, as Arthur Magazine editor Jay Babcock writes, "is one of the few mainstream entertainment industries open to folks who are openly into what is considered to be very weird, spooky and possibly dangerous stuff." 325 In fact, "comic books would seem to be the perfect medium for the exploration of magic and the occult." ${ }^{326}$ Occultist and esoteric traditions of thought often feature views of selfhood that are counter to the entrenched Western view associated with normative religious traditions, as mentioned above. So, comics is an apt medium for direct representations of these traditions, as well as convergent thematic content.

The terms "occultism" and "esotericism" defy simple or concise definitional encapsulations. ${ }^{327}$ These terms share much overlap and both tend to refer to sets of experiences or knowledge that usually bear strong resemblances to religious frameworks, but are hidden from the sphere of normative understanding or beliefs. The term "occult" signals the hidden nature of the beliefs and practices of these traditions and the term “esoteric" 'derives from the Greek word esotero, meaning 'within,' or 'inner.",328 Those following and practicing esoteric and occultist traditions often make "a claim to gnosis,

comics exemplify the notion of selves as constructed and interpreted narratives in a way that philosophers Nelson Goodman and Catherine Elgin would call "metaphorical" exemplification or "expression." The comic metaphorically exemplifies (or expresses) narrative selfhood because typical cases of human selfhood do not literally obtain within the comic, but the selfhood of characters is implied as being narrative in nature through readers interactions with the images and text.

${ }^{325}$ Jay Babcock, "Magic is Afoot: A Conversation with Alan Moore about the Arts and the Occult," Arthur, May 2003, https://arthurmag.com/2007/05/10/1815.

${ }^{326}$ Jason Lawton Winslade, "Enrolling in the 'Hidden School,"' in Supernatural Youths: The Rise of the Teen Hero in Literature and Popular Culture, ed. Jes Battis (New York: Lexington Books, 2011): 199.

${ }^{327}$ Dan Burton and David Grandy, Magic, Mystery, and Science: The Occult in Western Civilization, (Bloomington, IN: Indiana University Press, 2004), 3.

${ }^{328}$ Verslius, Magic and Mysticism, 1. 
or direct spiritual insight into cosmology or metaphysics.. ${ }^{, 329}$ Over the past few centuries, occultist and esoteric traditions have shown clear commonalities to the categories of religion, technology, and science. ${ }^{330}$ Examples of occultist and esoteric traditions include various types of mysticism, ceremonial magic, numerology, astrology, and others. An indepth exploration of any of these is beyond the scope of this work, but suffice it to say that these movements are often categorized as examples of broader occultist and esoteric movements. One feature that these traditions share is the perspective that the world is not as it appears or is commonly thought to be, particularly in relation to ourselves.

The expansion of the natural sciences and scientists' attempts to explain the world in mechanistic terms, "cast a hegemonic shadow over other intellectual endeavors during the late eighteenth century and throughout the nineteenth century. As a result, the occult, along with [certain traditions in] philosophy, theology, and art, suffered tremendous internal tensions. ${ }^{, 331}$ Those involved in occultist traditions addressed the question of whether and how to incorporate mechanistic scientific understandings of the world in various ways. In fact, some traditions that veered into occultist territory over their histories (such as mesmerism and phrenology) were thought by early practitioners to be burgeoning areas of natural science. ${ }^{332}$ This, in part, explains the potential for distinctions between more supernatural types of occultism and occultisms that are focused on extraterrestrial life, intergalactic/dimensional travel, and other concepts that, while fantastical, hypothetically would not contradict a scientific picture of the world.

\footnotetext{
${ }^{329}$ Verslius, 1.

${ }^{330}$ Burton and Grandy, Magic, Mystery, and Science, 4.

${ }^{331}$ Burton and Grandy, 184.

332 Burton and Grandy, 185.
} 
Occultist and esoteric traditions typically revolve around beliefs and practices that are secretive, or at least hidden from common cultural knowledge and belief. "Secrecy is [often] a mark of occult systems. ${ }^{, 333}$ But this is not a necessary feature of occultist or esoteric traditions, as "some have devotees who are happy to share their understanding with others. ${ }^{3334}$ Regardless of the role of secrecy, many of these traditions challenge the entrenched Western view of selfhood through their beliefs and practices.

For example, the practice of "mesmerism" (named for Dr. Dranz Anton Mesmer) was founded on the notion that universal "subtle magnetic fluids" ${ }^{335}$ compromised the life force of individuals and these fluids could be manipulated with the use of magnets in order to influence the healing of patients. Mesmer eventually "discovered that he could dispense with magnets in healing his patients. He could concentrate the magnetic fluids within his own body-use his own 'animal magnetism'-to act upon those within other bodies. ${ }^{״ 36}$ While classical mesmerism was pseudo-scientific, hypnotism (a concept with scientific legitimacy) was discovered when certain mesmerized individuals appeared to be sleeping but were still responsive to verbal suggestions. Mesmerism, as an occultist tradition, implied that we are not uncomplicatedly autonomous selves. Rather, the thoughts and behaviors that constitute our selves are dependent on impersonal forces below our conscious control (in this case, dependent upon subtle magnetic fluids).

Other occultist traditions also draw on the notion that there are forces outside of the immediate day-to-day realm of humans' experiences that can influence their selves. Many hold that through the magical use of symbols, words, and artwork, people can

\footnotetext{
${ }^{333}$ Burton and Grandy, 5.

${ }_{334}^{334}$ Burton and Grandy, 3.

${ }^{335}$ Burton and Grandy, 186.

${ }^{336}$ Burton and Grandy, 187.
} 
shape and determine themselves. These esoteric traditions mirror closely the views of those philosophers discussed in the previous chapter, who claim that our selves are interpretive narrative achievements that we use "as a part of a theoretical apparatus to understand, and predict, and make sense of, the behavior of some very complicated things. ${ }^{, 337}$ In narrative views of selfhood and also in many esoteric and occultist traditions, humans use words and symbols to represent, interpret, and narrativize the meanings of the their experiences and their self-conceptions.

Those who practice the tradition of numerology, for example, assert that there are hidden meanings in the relationships between alphabetical and numerical symbols. So numerical digits, rather than being only arbitrary symbols that are useful in tracking abstracted quantities, are able to reveal the meanings associated with personalities and situations. Similarly, Tarot cards are utilized in various occultist traditions in service of self-interpretation and the articulation of personal meaning. Practitioners utilize the artwork on the Tarot cards to perform ritualistic acts of what Daniel Dennett would call "auto-hermeneutics," or the ability "to go back and think about one's past, and one's memories, and to rethink them and rewrite them." ${ }^{338}$ This manifests through the use of Tarot cards when, as media theorist James Frost writes, "the world of the cards meets our own world in an encounter which is in constant motion, [and practitioners use the artwork to] endlessly revise our understanding. ${ }^{\text {} 339}$ These occult traditions involve the belief that symbols enable practitioners to develop and uncover meaning and that the

\footnotetext{
${ }^{337}$ Dennett, "The Self as a Center of Narrative Gravity," http://cogprints.org/266/1/selfctr.htm.

${ }^{338}$ Dennett, http://cogprints.org/266/1/selfctr.htm.

${ }^{339}$ James Edward Frost, "The Serious Game: Towards a Hermeneutic Understanding of the Tarot," The International Journal of the Image 7, no. 2 (2016): 25.
} 
interrelationships of these visual representations can allow people to understand their selves more deeply.

In this previous section, I have detailed the common features of esoteric and occultist traditions, as well as the etymological implications that such traditions involve secretive, hidden, and potentially subversive beliefs and practices. These traditions are often religiously resonant but need not involve supernaturalism, as some such traditions reference concepts that are potentially compatible with the natural sciences (such as, extraterrestrial beings, interdimensional travel, and attempted manipulations of human psychology). Esoteric and occultist traditions often promote understandings of human selfhood as malleable, interpretable, and responsive to various representations (such as, symbols, images, and text). And the experience of the renegotiation or dissolution of the typical perceived boundaries between self and non-self is often framed by such traditions as desirable or admirable.

The format of the sequential artwork in comics (as discussed in previous chapters) functions in a similar way, allowing readers to conceptualize the selves of characters and their own selves by inferring and imagining coherence across distinct and discreet images. Several of the prominent genres typically associated with the comics medium exemplify the tendency for comics stories to undermine the entrenched Western conception of selfhood, including the most popularly associated genre: the superhero story.

Superheroics and Narrative Identity

Comic books, as we know them now, were pre-dated by self-contained collections of newspaper comic strips and cheaply manufactured pulp fiction magazines in the early 
twentieth century. While comics have always contained stories of various genres, superhero stories are certainly the narrative genre most popularly associated with the comics medium. This common association began in the 1930's. As comics theorists Duncan and Smith write, "the superhero is recognized as a particularly American creation and is often seen as an embodiment of American ideology." ${ }^{340}$ Superhero stories, as a genre, "are the ultimate amalgams, all-swallowing über-characters that consume other genres like black holes. ${ }^{, 341}$ As such, superhero stories often contain conventions of adjacent narrative genres, including adventure, science fiction, and horror.

While the term "superhero" dates to years before his creation, ${ }^{342}$ Superman is unquestionably the paradigmatic superhero. Capes and crime fighting are typical tropes of superheroes, but comics scholars have gone further in determining the requirements for a character or narrative to be considered as part of the superhero genre. The superhero genre is defined, argues comics scholar Peter Coogan, by the themes of mission, powers, and identity. Superheroes have a purpose for their actions that is "pro-social and selfless," ${ }^{343}$ they have exaggerated abilities, and a "codename and costume, with the secret identity being a customary counterpart to the codename." ${ }^{\prime 34}$ This dual identity conceit is one way in which superhero stories highlight the narrativized nature of the selves of comics characters.

${ }^{340}$ Duncan and Smith, The Power of Comics, 243.

${ }^{341}$ Chris Gavaler, On the Origin of Superheroes: From the Big Bang to Action Comics No. 1 (Iowa City: University of Iowa Press, 2015): 3.

${ }^{342}$ Gavaler, 6.

${ }^{343}$ Peter Coogan, "The Definition of the Superhero," in Super/Heroes: From Hercules to Superman, eds. Wendy Haslem, Angela Ndalianis, and Chris Mackie (Washington, DC: New Academia Publishing, 2007): 24.

${ }^{344}$ Coogan, 25. 
The notion of the dual or secret identity ${ }^{345}$ has been part of the superhero genre at least since Superman first appeared on newsstands in 1938 (and arguably for decades before). ${ }^{346}$ The secret identity trope of superhero stories is probably the most obvious way in which this prominent genre of comics narratively undermines the entrenched view of selfhood. Superheroes typically embody at least two separate, and often contradictory or mutually exclusive, selfhood narratives. Superman, the brave and iconic hero, also lives out the identity of Clark Kent, the bumbling and timid reporter from Kansas. Batman is a shadowy, meticulous, and secretive vigilante waging a war on organized crime in Gotham City. But, he is also Bruce Wayne, a billionaire whose interests oscillate between highly publicized philanthropy and mindlessly self-indulgent buffoonery.

The fact that superheroes often have dual identities highlights to readers that much of the characters' (as well readers' own) identities are constructed through cultural expectations and conventions. If Bruce Wayne were to punch criminals in the face in broad daylight while wearing a three-piece suit, he would not only divulge his secret identity and thereby allow his enemies to more effectively threaten him, he would also be defying the constructed social narratives of selfhood that we tend to recognize in the mythology of the Batman. Selves, being considered here as narrativized abstractums, ${ }^{347}$ in part, are constructed by the social performance of the individual with whom the self is associated. "We play different roles in the world [and] we often go into a different mode

\footnotetext{
${ }^{345}$ It should be noted that dual identities are not necessarily secret identities. Marvel comics has typically relied less on secretive dual identities for their characters. For example, Steve Rogers is Captain America and this fact is publically known in the storyworld of Marvel comics.

${ }^{346}$ Gavaler, On the Origin of Superheroes, 5.

${ }^{347}$ Dennett, "The Self as a Center of Narrative Gravity," http://cogprints.org/266/1/selfctr.htm. Again, here "abstractums" refers to the fact that selves are conventional constructions that lack a continuous and unified essentiality.
} 
of self-identity and self-presentation in order to perform [them] well.."348 In this way, the superhero's multiple identities communicate to readers that selves are constituted (at least in part) by the ways in which an individual interacts with, and presents herself to, her environment.

This is not to suggest that everyday behaviors that signal particular social roles necessarily constitute a shift in one's entire identity or selfhood. For example, the doctor who wears a white coat and stethoscope is not a different self from who she is when she wears clothes that are not occupation-specific. However, the exaggerated versions of this type of performance of selfhood found in superhero narratives potentially undermine the notion that there is some unambiguous singular stable self that exists both in and out of the superhero's costume, and brings into focus the question of the role that readers' own social performances play in constituting their selves.

For example, is Superman's behavior brave? Certainly. But Clark Kent's behavior is cowardly. Similarly, Batman is the paradigm of self-discipline, while Bruce Wayne is highly self-indulgent. However, Batman and Bruce Wayne are one and the same, as are Superman and Clark Kent. These distinctions between the characterization of Batman and Bruce Wayne, and Superman and Clark Kent, are intelligible because these comics characters with dual identities manifest multiple and often contrasting personas. These personas, or personalities, are malleable social conventions. In the view of philosophers who hold to a narrative self view, selves in the actual world are similarly flexible constructions. As Jeffrey Kripal writes:

\footnotetext{
${ }^{348}$ Tom Morris, "What's Behind the Mask? The Secret of Secret Identities," in Superheroes and Philosophy: Truth, Justice, and the Socratic Way, eds. Tom Morris and Matt Morris (Peru, IL: Carus Publishing, 2005): 252.
} 
The imagined figures of Batman and Superman are indeed fictions. But so too is the imagined figure of your ego. Egos are interchangeable, malleable, plastic, and finally related to every other in the ground of being. They are like the suits put on and taken off by superheroes. They are costumes. They are masks (personas). ${ }^{349}$

Therefore, in one of the most common tropes of superhero stories we see the notion that certain elements of selfhood are not uncomplicatedly accounted for by the traditional Western view. This is fully intelligible in light of the implications of the formal features of comics discussed in previous chapters. The selves of comics characters, independently of narrative content, are presented as multiple and disunified on the standard comics page and coherence is enacted by the active engagement and interpretation of the reader. Comics writer Grant Morrison took the notion of the superhero's dual identity to its potentially occultist and mystical conclusions in his storyline Batman R.I.P.

Like much of Morrison's comics work, Batman R.I.P. has a dense plot that primarily serves to display the narrative themes that the author hopes to explore. This particular narrative pits Batman against a secretive theatrical organized crime group known as "The Black Glove," who hope to "ruin [Batman] in every way imaginable, body and soul, ${ }^{, 350}$ in order to herald in an era of unimpeded crime waves. A central antagonist named Dr. Hurt leads the Black Glove. The organization learns Batman's secret identity when one of its members poses as a love interest for Bruce Wayne. The group had implanted a psychological trigger word in Bruce's subconscious that his duplicitous love interest recites just before The Black Glove breaches the Batcave and assaults Bruce. He awakens later on the street in plain clothes, having no memory of his

\footnotetext{
${ }^{349}$ Jeffrey J. Kripal, Mutants and Mystics: Science Fiction, Superhero Comics, and the Paranormal (Chicago: The University of Chicago Press, 2011): 247.

${ }^{350}$ Grant Morrison, Batman R.I.P. (New York: DC Comics, 2010).
} 
identity. After wandering around the streets of Gotham for a day with a man who is apparently dead (again, the plot is dense), Bruce constructs a Bat-suit out of purple, red, and yellow cloth and begins referring to himself as "The Batman of Zur-En-Arrh."

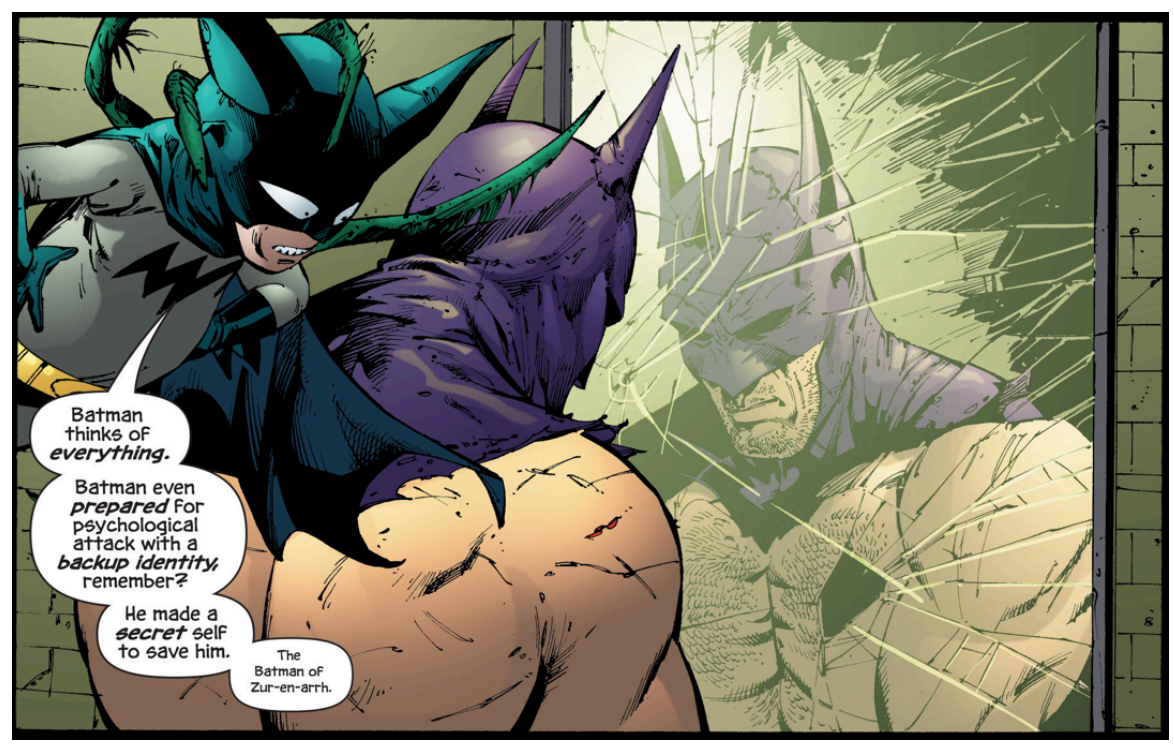

Figure 27: Grant Morrison, Batman R.I.P. (New York: DC Comics, 2010).

After putting on the multi-colored suit and becoming the Batman of Zur-En-Arrh, Batman begins to see and interact with a small fifth dimensional imp called "Bat Might" (a riff on a kid-friendly character from the 1950's Batman comics called Bat-mite). "ZurEn-Arrh" was the trigger word for the Black Glove's attack on Bruce's mind. But Batman, who characteristically over-plans for every possible threat, "prepared for a psychological attack with a back up identity." ${ }^{351}$ Therefore, rather than functioning as only a psychological weapon against Bruce Wayne, the phrase serves to activate "a secret self. ${ }^{, 352}$ This secret self, even more secret than the typical secret identity trope found in most Batman narratives, functions to highlight the malleable and constructed nature of Batman's selfhood.

\footnotetext{
${ }^{351}$ Morrison, Batman R.I.P.

${ }^{352}$ Morrison, Batman R.I.P.
} 
The Batman of Zurr-En-Arrh even goes so far as to distinguish himself from the typical Bruce Wayne/Batman self, saying, "I'm what you get when you take Bruce out of the equation. ${ }^{, 353}$ Of course, at the end of the narrative Batman resumes his typical dualistic self-narrative, but Morrison's construction of the Batman of Zurr-En-Arrh accentuates the potentially subversive implications of the dual identity trope for the concept of selfhood. Ultimately, there is no essential, continuous, or unified selfhood for Bruce Wayne/Batman, rather his self is (or selves are) narratively constructed in various ways, including social performance. Morrison makes explicit the conceptual similarities between having a secret identity and the notion that selves are narrativized, malleable, and conventional.

The secret identity trope is not the only way in which superhero stories have explored or featured selfhood in potentially subversive ways, and such explorations are not a late development of the superhero genre. One candidate for the first "superhero," or at least the superhero's "clearest progenitor" ${ }^{, 354}$ is Doctor Occult, ${ }^{355}$ a character featured in New Fun Comics (eventually entitled More Fun Comics). Doctor Occult is a freelance investigator who specializes in supernatural and occultist crimes and mysteries. Created by Jerry Siegel and Joe Shuster, the creators of Superman, Doctor Occult highlights the role that occultist traditions and their undermining of the common views of selfhood have played in the cultural history of American comics. Christopher Knowles, comics artist and writer, rightly notes that "it is highly significant that the character who becomes the

\footnotetext{
${ }^{353}$ Morrison, Batman R.I.P.

${ }^{354}$ Christopher Knowles, Our Gods Wear Spandex: The Secret History of Comic Book Heroes (San Francisco: Red Wheel/Weiser, 2007): 115.

${ }^{355}$ Knowles, Our Gods Wear Spandex, 117. Doctor Occult's name was later changed to Doctor Mystic.
} 
definitive archetype of the modern superhero is brought into the world by the same men who created the obscure 'Doctor Occult,' and that Superman bears such a strong, if unacknowledged, resemblance to his mystical progenitor., ${ }^{\text {356 }}$ Doctor Occult highlights the cultural and conceptual links between subversive religiously resonant themes and American superhero comic books.

Many examples highlight the commonality of plot points in the Doctor Occult stories that suggest an understanding of selves as non-essential and malleable. In the first example below, Doctor Occult has been visited in his bedroom by a "wraith-like figure," who tells Occult to go to a particular address at once. ${ }^{357}$ Upon arriving, a scientist (who was the wraith) tells Doctor Occult that his assistant has been lost in the "ether-world" and needs to be rescued. The narrative twist is that there is no assistant and the scientist's brother was convicted of murder because of Occult's work, prompting the scientist to take revenge. The scientist hopes to trap Occult in the ether-world forever. Of course Occult finds a way out of the sticky situation, but the point for our purposes is that the device of the ether-world serves to subtly undermine the entrenched Western notion of selfhood in favor of a concept of a selfhood without definitive boundaries.

The scientist in this story has the ability to appear as a "wraith-like" figure, implying that selves are not identical to the physical organisms with which they are associated. Also, there are several images of Occult and the scientist blending into the environment in the ether-world. The metaphysics of the ether-world obviously are not addressed in the brief narrative, but it is clear that the size of people may vary (e.g., the

\footnotetext{
${ }^{356}$ Knowles, 117.

${ }^{357}$ Leger and Reuths, "Doctor Occult: The Ghost Detective," in More Fun Comics no. 26 (New York: National Allied Publications, 1937).
} 
impossibly large scientist) and also that the typical boundaries of people are subverted and the characters blend seamlessly into their surrounding environment. These amorphous and hazy depictions visually undermine the distinction between self and nonself when Occult and the scientist are in the ether-world. ${ }^{358}$

${ }^{358}$ John L. Steadman, H.P. Lovecraft and the Black Magickal Tradition: The Master of Horror's Influence on Modern Occultism (San Francisco: Weiser Books, 2015). The early U.S. comics tradition is also culturally connected to the tradition of genre-based pulp fiction from the late Nineteenth and early Twentieth centuries. The horror and science fiction of H.P. Lovecraft, for example, has many points of convergence with various types of occulisms. Many of the philosophical conclusions reached here would likely be applicable to an examination of the relationship between prose fiction and notions of selfhood that are compatible with occultist and esoteric traditions. However, the key distinction in regard to comics (as discussed in detail in the previous three chapters) is the centrality and use of images and the way that the formal features shape the affordances of comics readers' experiences. 


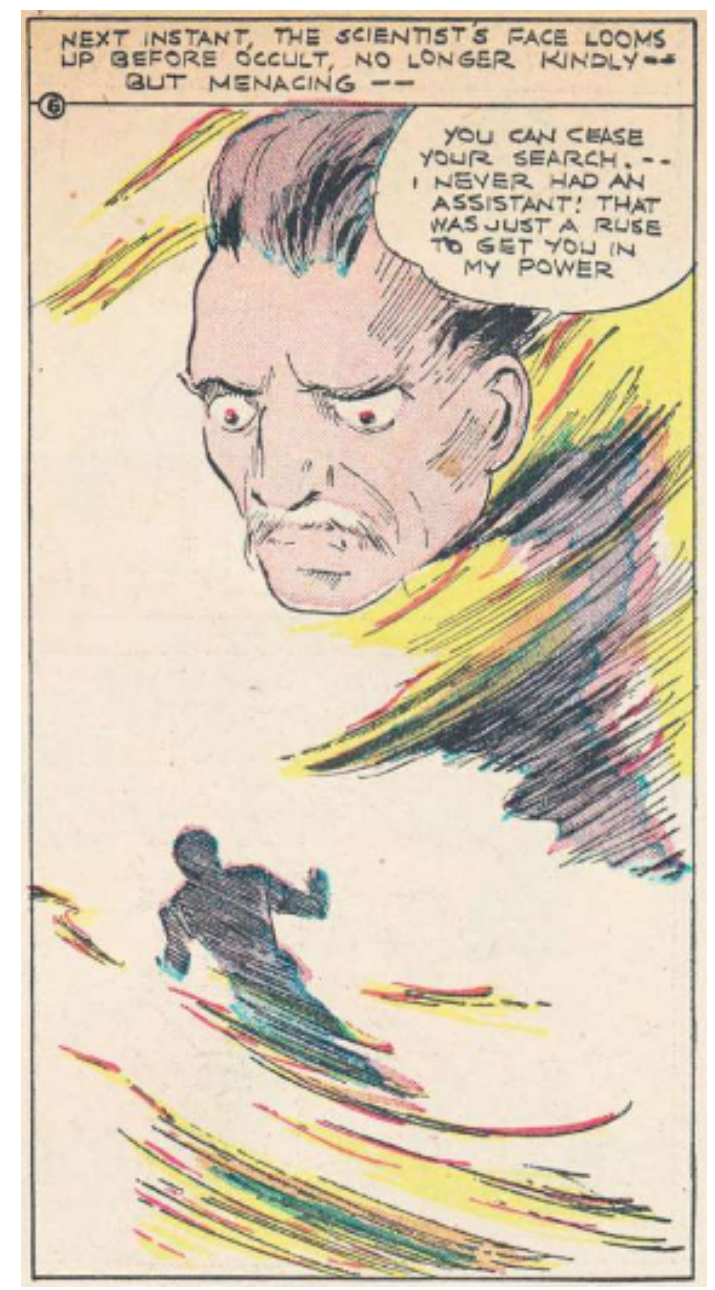

Figure 28: Leger and Reuths, "Doctor Occult: The Ghost Detective," More Fun Comics no. 26 (New York: National Allied Publications, 1937).

In a second example, the police summon Doctor Occult to help solve the mysterious deaths of three art critics who were judges at a recent art show. While the police and Occult question a fourth judge, the judge is attacked by an invisible wolf and dies with fang marks on his neck. Occult comes to realize that an embittered artist is murdering the judges through supernatural means by painting images of them dying in particular ways. The mechanism by which the artist's seemingly magical abilities function is not disclosed in the narrative, but this example is particularly interesting in that it implies not only that the lives of the art judges are literally narrativized by the murdering artist, but they are narrativized visually. 
In other words, certain artistically depicted events shape the self-narratives of the unfortunate art judges. It would be going too far to say that these early Doctor Occult comics offer a clear and coherent refutation to the entrenched Western view of selfhood, but several points within the plot certainly problematize a simple notion of an essential unified self. ${ }^{359}$ The fact that these themes regularly appear as early as 1937 in the stories of a character who was a key influence on the prototypical superhero can be understood in light of the implications of the formal features of the medium itself, rather than only as historical coincidence. That is, the medium of comics implies a multiple and constructed selfhood for characters and stories that blur the boundaries between self and non-self highlight this implication. A story with occultist themes that implies a malleable or potentially multiple selfhood for its characters has a natural home in comics.

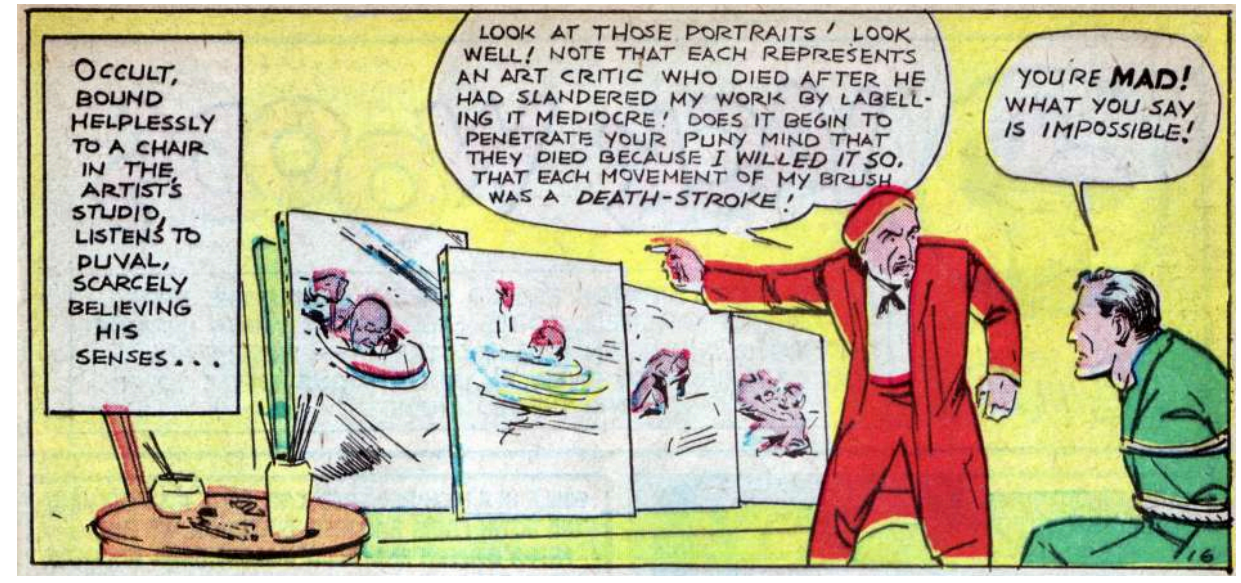

Figure 29: Leger and Reuths,

"Doctor Occult: The Ghost Detective," More Fun Comics no. 24

(New York: National Allied Publications, 1937).

${ }^{359}$ It is certainly possible that one could read these stories (and many of the following examples) and still hold to some type of amended or philosophically sophisticated essential or unified self view. The view of some phenomenologists that subjectivity has a certain quality of "mineness" that constitutes the true locus of selfhood (as was mentioned in the previous chapter) is not challenged by these examples, for instance. However, because comics function as artifacts of popular culture, rather than as academic treatises, the relevant point is that many comics implicitly undermine the common Western folk notion of a unified and essential self. And they do so by virtue of their formal features, not only the content of the stories they tell. 
The conflation of scientific and magical principles is another important and recurring theme that is on display in the previous examples from Doctor Occult. This conflation is apparent in many genre comics, as well as in occultist and esoteric traditions of thought that also emerged in the late Nineteenth and early Twentieth centuries. Many of those who engaged in potentially subversive spiritualist traditions sought to:

unite a scientific worldview with a religious one. Hence, with the invention of the telegraph and the discovery of electricity, the Spiritualist world was all abuzz with talk of 'vibrations,' 'frequencies,' the 'spectrum' of consciousness, a 'fourth dimension,' even a 'spiritual telegraph' connecting the living and the dead. The supernatural wanted to become scientific. ${ }^{360}$

This equivocation of science, occult and esoteric practices, and fantastical pseudo-science is found in both actual traditions of thought ${ }^{361}$ and also in the narratives of comic books from the early Twentieth century. This brings us to the implications for selfhood in science fiction comics.

Science Fiction, the Fantastical, and the Humbled Self

Science fiction stories have been a staple of American comics since the early Twentieth century. Newspaper comic strips that rose to popularity in the 1930's, such as Buck Rogers in the Year $25^{\text {th }}$ Century AD and Flash Gordon, largely determined Americans' understandings of the science fiction genre during the time. Science fiction historian Mike Benton writes, "so great an impact did Buck and Flash have on science fiction and on comics, it's tempting to see them as the sole progenitors of all science

\footnotetext{
${ }^{360}$ Kripal, Mutants and Mystics, 126.

${ }^{361}$ Burton and Grandy, Magic, Mystery, and Science, 5. "It is impossible to define the occult in a way that sets it completely apart from religion, science, and technology."
} 
fiction comics to come. ${ }^{״ 362}$ These early science fiction comics introduced many of the elements that have become associated with science fiction media more broadly. Some tropes of early science fiction comics include extra-terrestrial beings, interstellar and time travel, galactic wars, robotic intelligences, and alien terrains, although these tropes are neither necessary nor sufficient in classifying a narrative as science fiction. ${ }^{363}$

Science fiction comics often present wildly speculative visions of potential futures and alternate worlds in which the familiar becomes unfamiliar and the nature of humans, as well as our place in the universe, is examined and questioned. Many of these comics consist of adventure stories set in or involving outer space. The use of fantastic and futuristic locations allows apparently "straightforward and simpleminded"364 action to carry the potential for broader thematic implications. Benton compares this speculative function to that of religious prophesy, writing:

A science fiction comic book story taps into the primal dream state with its exaggerations, archetypes, and colorful images of 'worlds other than our own.' Reading a science fiction comic is like being privy to a shamanistic prophesy. You divine the 'future' (and, hence, the present) from filtered cartoon images played out in a timeless story of heroics and survival. ${ }^{365}$

Science fiction comics offer readers chances to think through fantastical alternative realities, which can potentially shed light on their understandings of the actual world.

\footnotetext{
${ }^{362}$ Mike Benton, Science Fiction Comics: The Illustrated History (Dallas, TX: Taylor Publishing Company, 1992): 14.

${ }^{363}$ Gwyneth Jones, "The Icons of Science Fiction," in The Cambridge Companion to Science Fiction, eds. Edward James and Farah Mendlesoh (New York: Cambridge University Press, 2003): 163. "The feature that unites every kind of [science fiction] is the construction - in some sense - of a world other than our own. This may be another planet (or even another universe); or it may be a 'future world' in which conditions have changed in some dramatic way."

${ }^{364}$ Benton, Science Fiction Comics, 5.

${ }^{365}$ Benton, 5.
} 
Futuristic and speculative narratives allow the readers of science fiction comics to reflect on themselves and their present circumstances through the lens of other worlds.

One example of science fiction comics relating the otherness of outer space with the destabilization of the common Western notion of human selves is found in the 1950's comics title Weird Science. Weird Science was published from 1950-1952, lasted 22 issues, and involved stories that were "carefully plotted to deliver a surprise-twist ending with a heavy helping of irony and cynicism." ${ }^{366}$ Like many comics titles by the same publisher (E.C. Comics), Weird Science "dared to make their readers think and question some of their most basic beliefs. ${ }^{\not 67}$ This tendency toward subversion was particularly potent in the mid-century American culture that valued order, homogeneity, the status quo, and social conformity so heavily. While many issues of Weird Science explored cultural and social norms, they also often implicitly undermined the common Western notion of human selves as unified, enduring, and essential.

\footnotetext{
${ }^{366}$ Benton, 38.

${ }^{367}$ Benton, 44.
} 


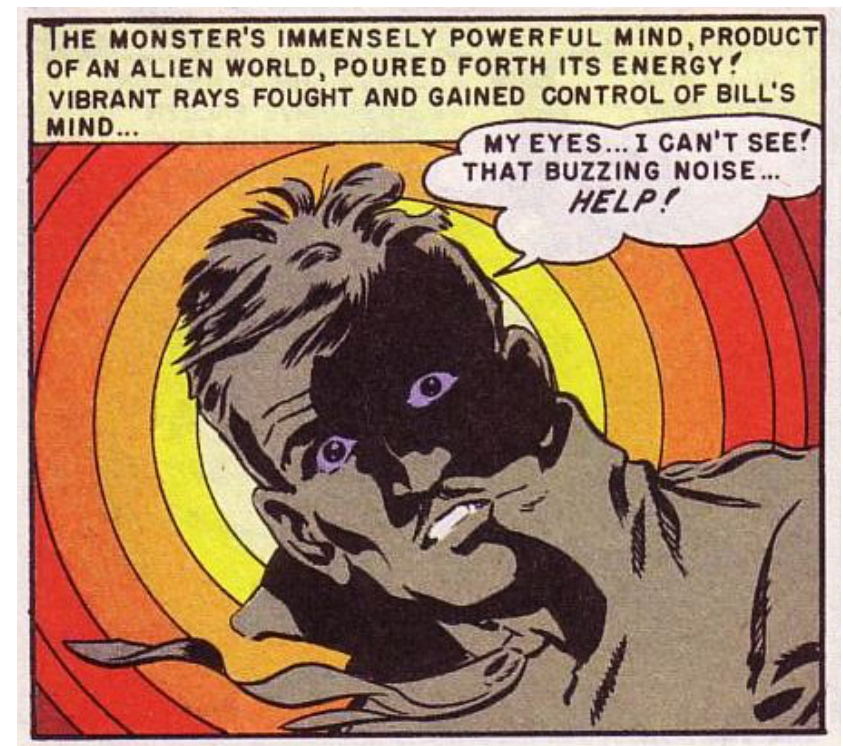

Figure 30: "The Meteor Monster," Weird Science \#13 (New York: Fables Publishing Company, 1950).

Issue \#13 of Weird Science contains a story entitled "The Meteor Monster" in which a meteor crashes next to a couple's house in an unidentified (presumably American) town. The man, Bill Manning, goes to investigate the meteor and encounters a small green alien creature with "queer eyes." ${ }^{368}$ The alien uses its glare to take telepathic control of Bill's mind and convinces Bill that he's friendly. Bill takes the alien back to his home, where his wife is then brought under the alien's telepathic control, followed by two friends who come to the Mannings' home to investigate the "terrific flash of light." ${ }^{\prime 39}$ The alien takes control of all of them and has them take it to a town meeting, at which point it takes control of all of the citizens at town hall.

\footnotetext{
368 "The Meteor Monster," Weird Science \#13 (New York: Fables Publishing Company, $1950)$.

369 "The Meteor Monster," Weird Science \#13.
} 


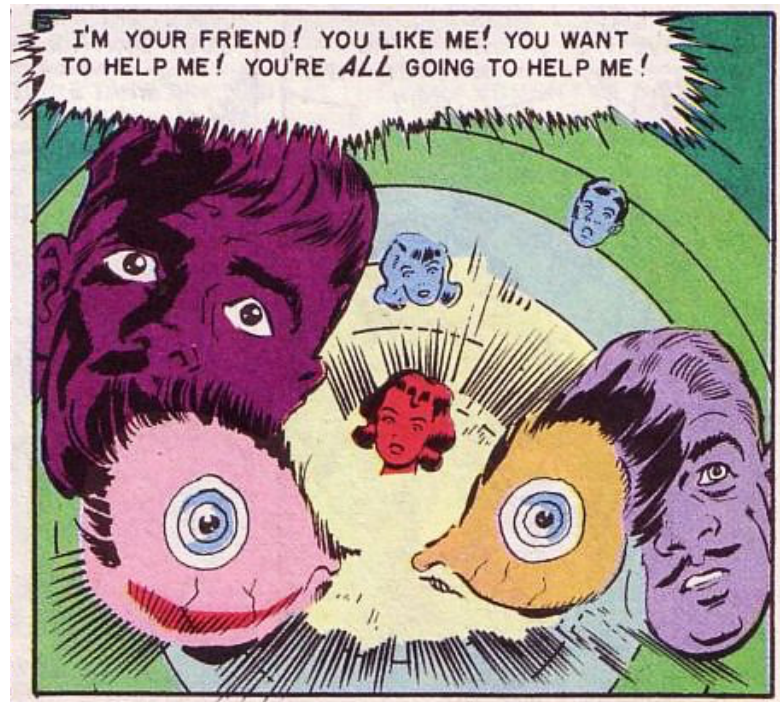

Figure 31: "The Meteor Monster," Weird Science \#13 (New York: Fables Publishing Company, 1950).

At the climax of the story, the telepathic monster encounters a blind man and its "mind energy cannot enter through his eyes," allowing him to attack the alien and free his fellow citizens from the alien's telepathic control. ${ }^{370}$ The relevant point for my purposes is the narrative trope and visual representations of mind control. Telepathy and mind control are staples of both science fiction comics, such as this one, and also of occultist and spiritualist movements. ${ }^{371}$ In Weird Science's "The Meteor Monster," the alien conforms the thoughts and behavior of the townspeople to its own will, highlighting the porous and malleable nature of the selves of those characters.

Additionally, the artwork visually represents the telepathic encroachment of the alien into the selves of the citizens at the town hall meeting. In Figure 31, the alien's eyes are depicted prominently in the center of the panel and some faces of the townspeople are depicted blending into the eyes of the monster. The monster's intrusive thoughts are

\footnotetext{
370 "The Meteor Monster," Weird Science \#13.

${ }^{371}$ Kripal, Mutants and Mystics, 54. Named by Frederic Myers in 1882, the concept of "telepathy" reflects Myers' spiritualist belief that "the subliminal Self beneath the threshold of normal awareness [...] is the true subject of psychical perceptions, the possessor of supernormal powers, and the survivor of death's grisly decay."
} 
presented in a thought bubble with harsh, jagged edges, suggesting the ominous nature of the telepathic undermining of the citizens' autonomy. The selves of the citizens become (in thought and action) simply extensions of the will of the alien monster from outer space, and they are visually depicted as intertwining with the alien's eyes. The townspeople are literally bent to the vision of the alien. However, while "The Meteor Monster" features the terrors of outer space coming to the Earth, other science fiction comics depict humans (or human-like characters) venturing into dangerous regions of outer space.

Consider the 2015 science fiction noir series Roche Limit published by Image Comics. It takes place primarily in the "Roche Limit colony" on the planet of Dispater, which sits on the "cusp of an energy anomaly in the Andromeda Galaxy." ${ }^{\text {372 }}$ The narrative explores the implications for humanity's understanding of itself in the face of intergalactic travel and the vastness of outer space. While Roche Limit features many standard narrative tropes of both the noir and adventure genres, including a morally ambiguous characters, a crime-riddled underworld, and space ship battles, it also deals in philosophical themes of human identity and selfhood in relation to the enormity of an indifferent cosmos.

The antagonist of Roche Limit is a super-organism with telepathic powers and a hive mind called "the Black Sun" that gains its power from the ingestion of human souls. Souls are visually represented as glowing white orbs (Figure 32), and are referred to as humans" "essence, their being." ${ }^{, 373}$ This view of human souls reflects heavily the entrenched Western view discussed in detail in the previous chapter, while the formal

\footnotetext{
372 Michael Moreci, Roche Limit Volume 1: Anomalous (Image Comics, 2015).

${ }^{373}$ Moreci, Roche Limit Volume 1: Anomalous.
} 
features of Roche Limit's narrative medium simultaneously undermine that same view of selfhood.

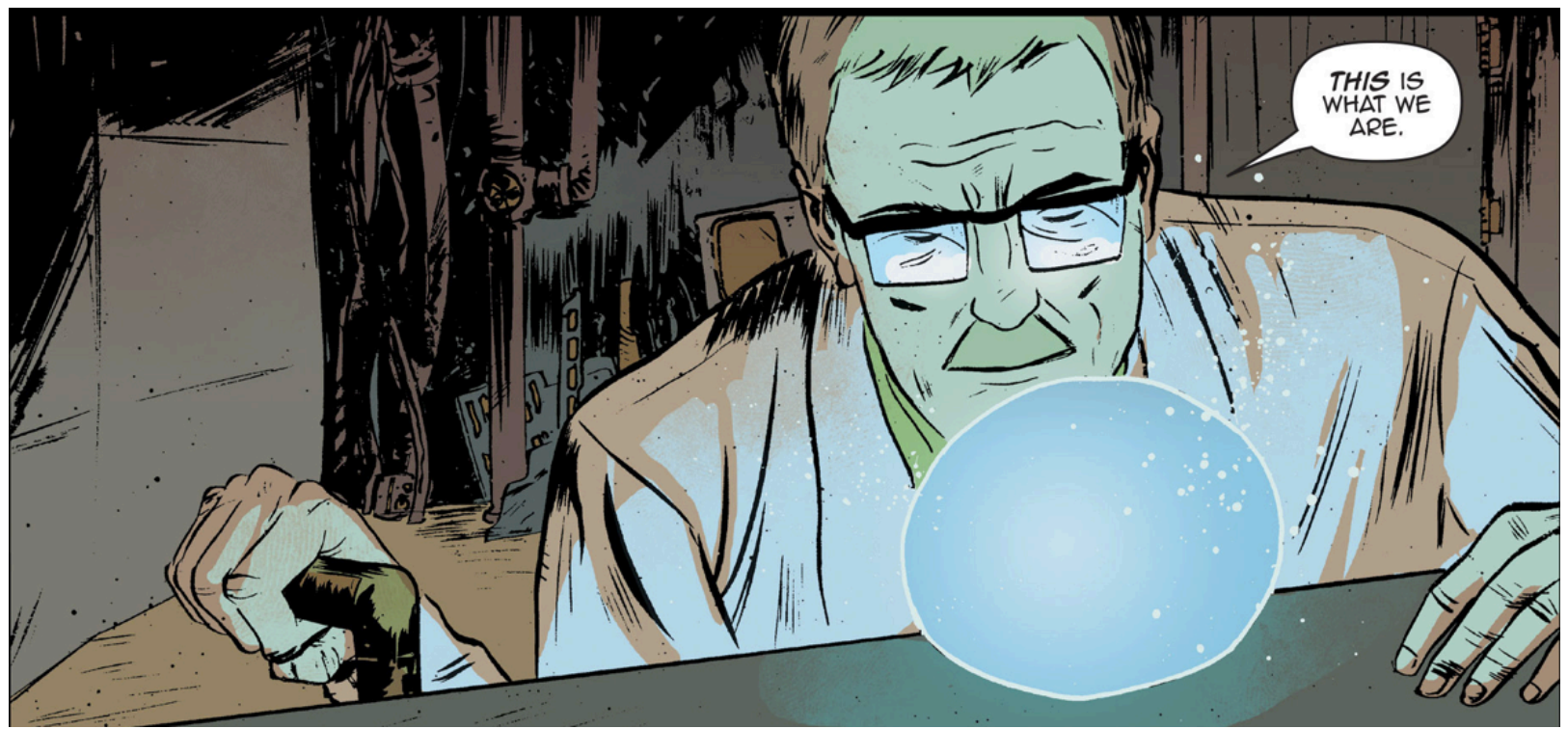

Figure 32: Moreci, Roche Limit Volume 1: Anomalous (Image Comics, 2015).

However, even in this comic series where something closer to the standard view of selfhood is visually represented (Figure 32), a view of selves as narratives is referenced as well. The founder of the Roche Limit Colony, Langford Skaargred, was an idealist who believed that "to understand [humans'] existence, to understand why we're here, we have to contextualize our role in the entirety of creation." 374 Skaargred founded the Roche Limit Colony as a manifestation of his optimistic outlook that space exploration would be the next pinnacle of human achievement. But the narrative of Roche Limit complicates and contradicts this picture by depicting Skaargred's colony as a crime-ridden landscape filled with noir tropes.

The images below (Figure 33), include Skaargred's understanding of human history and human selfhood as a process of narrativizing. Skaargred says "I've traveled the Cosmos, been awed and humbled by the Universe; I've experienced the closest thing

${ }^{374}$ Moreci, Roche Limit Volume 1: Anomalous. 
to transcendence that even I could hardly dream was possible. And I've learned. I've learned that everything we know, everything that we are... is an interpretation" [emphasis added]. ${ }^{375}$ So, even in this science fiction comics series that visually represents a "soul," contains the influence of the narrative view of selves in both formal and narrative terms. Humans selves are, according to this character who has explored the reaches of outer space, interpretations.

${ }^{375}$ Moreci, Roche Limit Volume 1. 


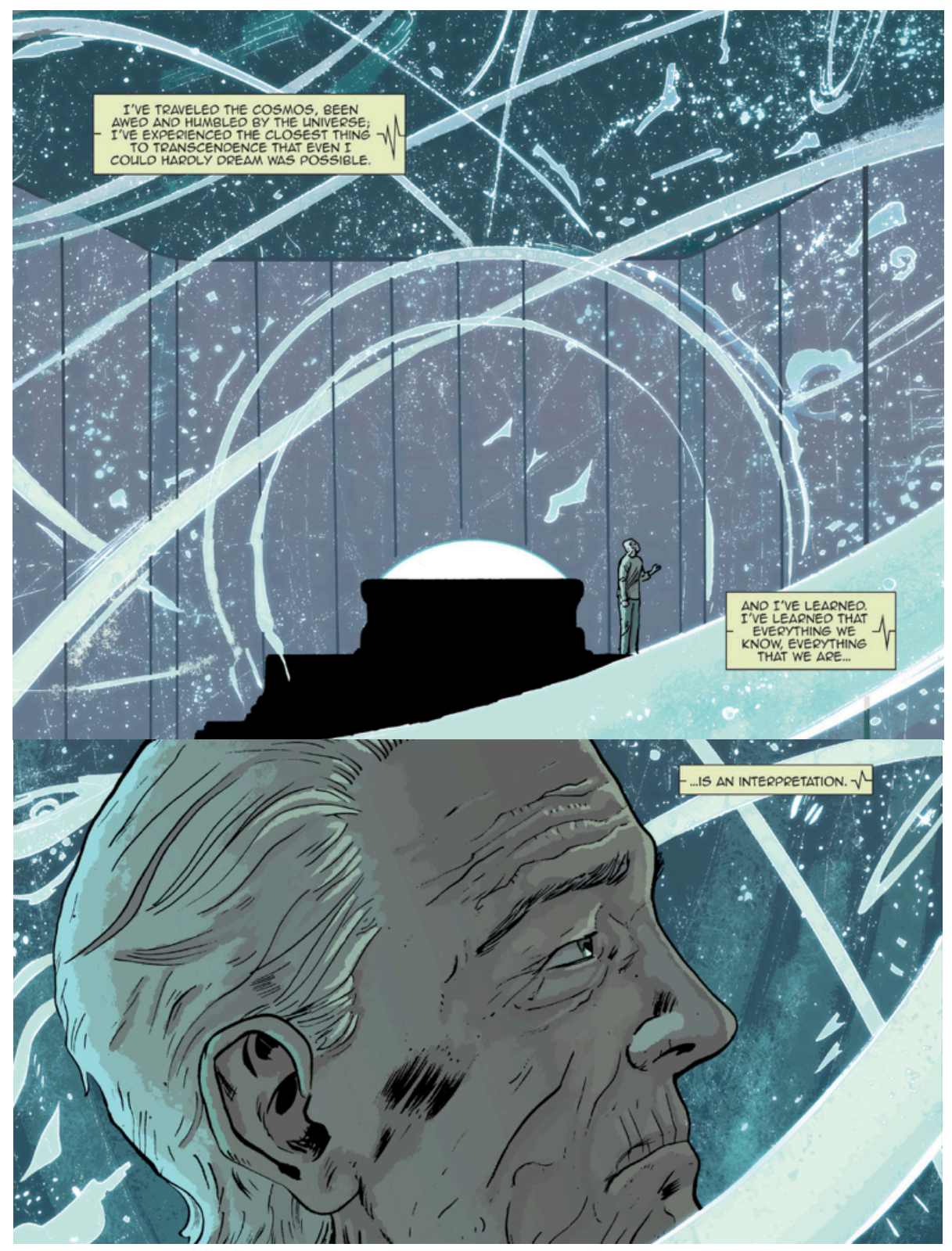

Figure 33: Moreci, Roche Limit Volume 1: Anomalous.

Both early and current science fiction comics often feature protagonists who adventure into the uncharted territories of outer space. While these stories can be read as an affirmation a type of space-aged manifest destiny, with a brave humanity aiming to conquer cosmic frontiers (e.g., the character of Langford Skaargred), these science fiction comics also highlight the understanding that the universe is filled with environments that are not suitable for human beings. In these spaces, humans are alien, out of place, and 
insignificant. The human self is decentered in narratives and artwork that place the human characters in non-human worlds. Hostile and unfamiliar extraterrestrial landscapes and creatures often dwarf the human characters, highlighting their fragility and transience in the face of an awe-inspiring cosmos filled with existential threats. This perspective of the world, which displaces humanity from the role of central importance, became more popularly understood with advances in the physical and astronomical sciences dating back at least a century. ${ }^{376}$ Jeffrey Kripal, writes:

Already in the early Twentieth century, the human being no longer lived in the center of the Universe, but on a minor planet orbiting a minor (gulp, dying) star somewhere on the outer arm of an average galaxy, of which we would soon learn that there are billions. The cosmos was now so unspeakably vast, its physics so utterly mindbending, that individuals were simply no longer capable of processing everything $[\ldots]$ The human being had, in a very profound sense, lost a home and, with it, a sense of belonging. Everything, literally everything, had become fundamentally alien. ${ }^{377}$

An increasing scientific understanding of the unimaginable scope of the universe was undermining the perceived cosmic importance of human selves during, and even preceding, the widespread popularity of comics. And the influence of this perspectival shift can be seen in the stories contained in science fiction comics.

In fact, the decentering of the human has gone beyond the increasing realization that the cosmos appears to be indifferent to the existence of human selves. Philosopher Robert McCauley writes of the tendency of the developing scientific picture of the world to continually "look beyond appeals to agent explanations to theories cast in terms of

${ }^{376}$ Brian Stableford, "Science Fiction Before the Genre," in The Cambridge Companion to Science Fiction, eds. Edward James and Farah Mendlesohn (New York: Cambridge University Press, 2003): 16. The shift from a geocentric to heliocentric understanding of the solar system centuries before was also influential in fantastical and pre-science-fiction stories, as well as being religiously and culturally subversive.

${ }^{377}$ Kripal, Mutants and Mystics, 71. 
radically counterintuitive representations of impersonal things and forces (from Thales's hydraulic account of physical phenomena to Democritus's claims on behalf of atoms)."”778 This means that not only has the human self become increasingly displaced from its presumed place of central importance because of the scientific picture of the world, but common entrenched ideas about the nature of selves have also been undermined. McCauley writes:

Now it appears to be psychology's turn. Over the last four decades the cognitive and psychological sciences have provided increasingly detailed accounts of cognitive operations that $[\ldots]$ repay more of the "intelligence loans" that constitute the very foundations of the maturationally natural conception of agency. In addition, machinery, such as parallel distributed processing networks, has proven capable of solving more and more of the complicated problems that, when humans solve them, we treat as intelligent accomplishments - precisely the sorts of intelligent accomplishments that we have always uniquely assigned to agents. ${ }^{379}$

So, science fiction stories that expand this scientific picture of selves and of the world in fantastical ways are particularly suitable to the comics medium, which formally implies a non-essential, divisible, and narrativized understanding of the nature of selves. And many science fiction narrative tropes share cultural overlap with non-normative traditions of occultist and esoteric thought because "many of the extraordinary capacities that science fiction and superhero comics treat as fantasies [telepathy, precognition, psychokinetic or magical influence, subtle bodies and energies, cosmic unity, and clairvoyance, to name the most common] are well-documented experiences in the history of folklore, religion, and psychical research." ${ }^{380}$ While science fiction comics undermine the common notion of selfhood through depictions of alien terrains, intelligent

\footnotetext{
${ }^{378}$ Robert McCauley, Why Religion is Natural and Science is Not (New York: Oxford University Press, 2011): 117.

${ }^{379}$ McCauley, 117.

${ }^{380}$ Kripal, Mutants and Mystics, 6
} 
machines, ${ }^{381}$ extraterrestrial beings, and the vastness of outer space, horror comics also often contain content dealing in subversive traditions of thought and themes of selfhood. And the cultural history of comics in the U.S. contains notable incidents of subversive horror comics, and the comics medium more generally, drawing fire from censors and critics.

Horror Comics, Youth Culture, and Subversive Values

The popularity of comics reached a fever pitch in the U.S. during and after the Second World War. According to surveys conducted in the early 1940's, "95 percent of children aged eight to eleven and 84 percent of children aged twelve to seventeen read comics [suggesting that] comic books had clearly emerged as a significant American leisure-time activity with particular appeal to children."382 The comic book industry increased offerings of horror and crime comics in the post-war U.S. in an attempt to capitalize on the new market of veterans, who had returned from the war. ${ }^{383}$ By 1953 , the Cincinnati Committee on the Evaluation of Comic Books "noted with alarm that nearly 50 percent of the [content of] comic books had become slightly or very objectionable," with the majority of objectionable material being found in horror related comics. ${ }^{384}$

The increase in comic books' sales and social capital during this time combined with "the explicit accusation that comic book reading was a factor in juvenile

\footnotetext{
${ }^{381}$ McCauley, Why Religion is Natural and Science is Not, 117.

${ }^{382}$ Bart Beaty, Fredric Wertham and the Critique of Mass Culture (Jackson, MS: University Press of Mississippi, 2005): 106.

${ }^{383}$ Carol L. Tilley, "Seducing the Innocent: Fredric Wertham and the Falsifications that Helped Condemn Comics," Information \& Culture 47, no. 4 (2012): 384.

${ }^{384}$ Bradford W. Wright, Comic Book Nation: The Transformation of Youth Culture in America (Baltimore: The Johns Hopkins University Press, 2001): 155.
} 
delinquency"385 ${ }^{38}$ rulted in the rise of a reactive anti-comics movement. ${ }^{386}$ The most vocal anti-comics crusaders included librarians, schoolteachers, religious leaders, and other figures of authority for the youth of the country. Concerns over comic books increased throughout the 1950's and 60's, thanks in large part to the political efforts of the German-born American psychiatrist Fredric Wertham.

Wertham is best known for his book Seduction of the Innocent, ${ }^{387}$ published in 1954, which detailed what he saw as the dangerous glorifications of violence and moral impropriety in emerging mass media, especially comic books. ${ }^{388}$ Seduction also detailed evidence $^{389}$ that Wertham purported to have collected himself, which he claimed verified the link between comic book reading and behavioral problems in children. While it would be grossly over-simplistic to paint all academics, parents, or educators of the post-war era as holding anti-comic book positions, ${ }^{390}$ Wertham and many others saw the violent imagery and subversive narrative content in horror comics as posing a threat to the psychological wellbeing, moral adjustment, literacy, democratic values, ${ }^{391}$ and aesthetic tastes of children in the U.S.

Those associated with the anti-comics movement had their detractors, and Wertham was often accused of suggesting an overly simplistic causal account of the

${ }^{385}$ Amy Kiste Nyberg, Seal of Approval: The History of the Comics Code (Jackson, MS: University Press of Mississippi, 1998): 18.

${ }^{386}$ Nyberg, 19. This concern was not, in itself, completely unreasonable "because evidence suggested crimes committed by children had increased significantly."

${ }^{387}$ Fredric Wertham, Seduction of the Innocent: The Influence of Comic Books on Today's Youth (New York: Rinehart, 1954).

388 Tilley, "Seducing the Innocent," 383.

${ }^{389}$ Beaty, Fredric Wertham and the Critique, 138. This research evidence was built on "multiple approaches" including observation, standardized tests, life history assessment, and clinical interviews.

${ }^{390}$ Beaty, 12.

${ }^{391}$ Wright, Comic Book Nation, 159. 
delinquency of youth. However, it is important not to exaggerate the simplicity of his view. Wertham did not claim that there was a "direct, linear relationship between reading comic books and delinquent behavior," rather "his point was that comic books were part of the social world of children and should not be dismissed as harmless entertainment."392 He sought to focus his psychiatric work on the often-unconsidered social influences (in this case, popular narratives in the form of comic books) that shaped children's moral and aesthetic sensibilities and behavioral dispositions.

The anti-comics movement, of which Wertham was a figurehead, condemned comics on aesthetic grounds, among other things. Comics were considered a crude and useless form of artistic expression. The visual artwork in comics was criticized as being of low quality and of lacking in aesthetic value. ${ }^{393}$ Children's enjoyment of this type of lowly art, it was said, threatened a "decline in artistic appreciation, and [an increased] taste for shoddy, distorted representations." ${ }^{394}$ While the conclusions drawn seem dubious in hindsight, many (though not all) early-to-mid-century comics did contain low-quality artwork churned out by “desperate, underpaid kids and sleazy entrepreneurs.",395

Anti-comics activists also denounced the literary value of comics. Comics were seen not only as lacking in literary worth themselves, but also as undermining readers' capacity for a well-calibrated aesthetic sensibility with regard to other literature. "Echoing earlier critics, Wertham believed that comic books prevented children from

${ }^{392}$ Nyberg, Seal of Approval, 86.

${ }^{393}$ Nyberg, 11. Many adults of the time perceived comics as "crude, simplistic, and lacking in any literary or artistic merit."

${ }^{394}$ Paul Witty, Ethel Smith, and Anne Coomer, "Reading the Comics in Grades VII and VIII," Journal of Educational Psychology 33 (1942): 181.

${ }^{395}$ Wolk, Reading Comics, 5. 
developing an appreciation for good literature. ${ }^{, 396}$ So, this popularized hybrid artistic form ${ }^{397}$ was thought to embody the worst of both the artistic disciplines from which it derived.

Aside from the question of the negative aesthetic effects of comic books, anticomics crusaders were also critical of what they saw as the ethical consequences of the narratives presented in comics. This concern was not kindled in a vacuum, as comics publishers $d i d$ "[veer] into increasingly mature, dark, and gory thematic territories"398 preceding the rise of the anti-comics movement. Wertham claimed that children's exposure to narratives glorifying characters engaged in morally dubious or vicious actions and situations encouraged children to mimic the behavior of those characters. He was especially critical of the "gratuitous linking of violence and sexuality" 399 in the comics he encountered. Wertham's argument hinged on the claim that the masses, especially children, would imitate characters depicted as protagonists in popular narratives.

Wertham maintained that children, being young and impressionable, were influenced in their moral sensibilities by cultural and societal factors, including comics. He "suggested that identification, the emotional aspect of reading, was corrupted by an ongoing confusion in most crime comics between the hero and the villain. He noted that comic books were conditioning children to identify with the strongest character in a given

\footnotetext{
${ }^{396}$ Nyberg, Seal of Approval, 94.

397 Aaron Meskin, "The Ontology of Comics," in The Art of Comics: A Philosophical Approach, eds. Aaron Meskin and Roy T. Cook (Malden, MA: WileyBlackwell, 2012): 38. I use the term "hybrid," not in order to take a stance on the ontology of comics, but to tease out the various angles of criticism leveled by the anti-comics movement.

398 Tilley, "Seducing the Innocent," 389.

399 Tilley, 393.
} 
story, 'however evil he may be." "400 Wertham extrapolated from the claim that impressionable children are likely to manifest delinquency because they mimic popular characters to the further claim that this constituted a broader public danger. ${ }^{401}$

Wertham, and others from the anti-comics movement, did not only seek to inform parents about the potentially problematic content of the comics their children were reading, but also to take censorious legislative action against the comics industry. Wertham testified before a Senate Subcommittee on Juvenile Delinquency in hopes of promoting legal censorship on the comic book industry. ${ }^{402}$ When critiqued based on considerations of freedom of speech, he retorted, "Nonsense. We are dealing with the mental health of a generation." ${ }^{403}$

Wertham focused on the relationship between the well being of society and psychological health of individuals. On Wertham's view, comics necessitated legal restrictions because they "conspired to seduce and betray America's youth and indoctrinate them with corrosive values." ${ }^{, 404}$ A censorious broadening of scope appeared in the anti-comics movement, in which "crusades against comic books were often begun with the idea of eliminating from the newsstand those comics containing graphic violence or sex, [but] quickly expanded their goals, monitoring the cultural and moral values depicted in the pages. $" 405$ In other words, anti-comics crusaders saw comics as so

\footnotetext{
${ }^{400}$ Beaty, Fredric Wertham and the Critique, 139.

${ }^{401}$ Duncan and Smith, The Power of Comics, 275.

${ }^{402}$ David Hadju, The Ten-Cent Plague: The Great Comic Book Scare and How it Changed America (New York: Farrar, Straus, and Giroux, 2008): 263-265.

${ }^{403}$ Hadju, 102.

${ }^{404}$ Beaty, Fredric Wertham and the Critique, 157.

${ }^{405}$ Nyberg, Seal of Approval, 30.
} 
subversive that though they began by targeting the representational content of the covers of comics, they ultimately targeted the content of the stories inside comics as well.

Wertham's warnings about comics for their tendency to "openly glorify crime",406 and "[give] murder prestige," ${ }^{407}$ suggest that his concern was not only with the depictions of immoral actions and characters, but also with the function they served in the narratives. That is, he was opposed to narratives depicting storyworlds that affirmed characteristics and behaviors that he viewed as subversive. The Comics Code Authority, which the comics industry imposed upon itself in the wake of Wertham's criticisms, essentially required the storyworlds that comic books depicted to operate according to the ideal of normative American values. As I have argued, however, the implications of the comics medium itself in the popular American context are potentially subversive, independent of narrative content.

The current scholarly consensus about Wertham's anti-comics crusade is that "the problems inherent in [his] analyses are obvious [because] in certain instances, Wertham clearly had misread or distorted the evidence." 408 It is also probable that the general panic about comics was, in part, due to the fact that " [children] who read crime and horror comics usually did so alone or in peer groups away from the company of adults,",409 which emphasized their growing independence. Regardless, Wertham cherry-picked examples to show comics in the worst possible light and did not specify which issues or titles his critiques were being leveled against, preferring to attack the medium as a whole.

\footnotetext{
${ }^{406}$ Fredric Wertham and Gerson Legman, "The Psychopathology of Comic Books," American Journal of Psychotherapy 50, no. 4 (1996): 418.

${ }^{407}$ Wertham and Gerson, 419.

${ }^{408}$ Wright, Comic Book Nation, 162.

${ }^{409}$ Wright, 163.
} 
However, one of the clear targets of Wertham's attacks was the definitive comics publisher, E.C.

E.C., which stands for Entertaining Comics, was the premier horror comics publisher during the early 1950's. With titles including The Vault of Horror, The Haunt of Fear, SuspenStories, Weird Fantasies, and Weird Science, ${ }^{410}$ it is "difficult to overstate how different E.C. comic books were from what came before them." ${ }^{, 411}$ E.C. comics were some of the highest quality of the time in terms of both artwork and narratives. They were written with teenagers and adult readers in mind, rather than aiming their narratives at children like most comics of the time. E.C. publisher William Gaines used the platform to "critique, satire, and subvert entrenched American values and institutions at a time when few other voices in popular culture did so." ${ }^{, 412}$ The subversion of American values in E.C. comics included not only political and social subversion, but implicit subversion of the entrenched view of selfhood that is the primary focus of this work.

${ }^{410}$ Discussed above (Figures 30-31).

${ }^{411}$ Wright, 135.

${ }^{412}$ Wright, 136. 


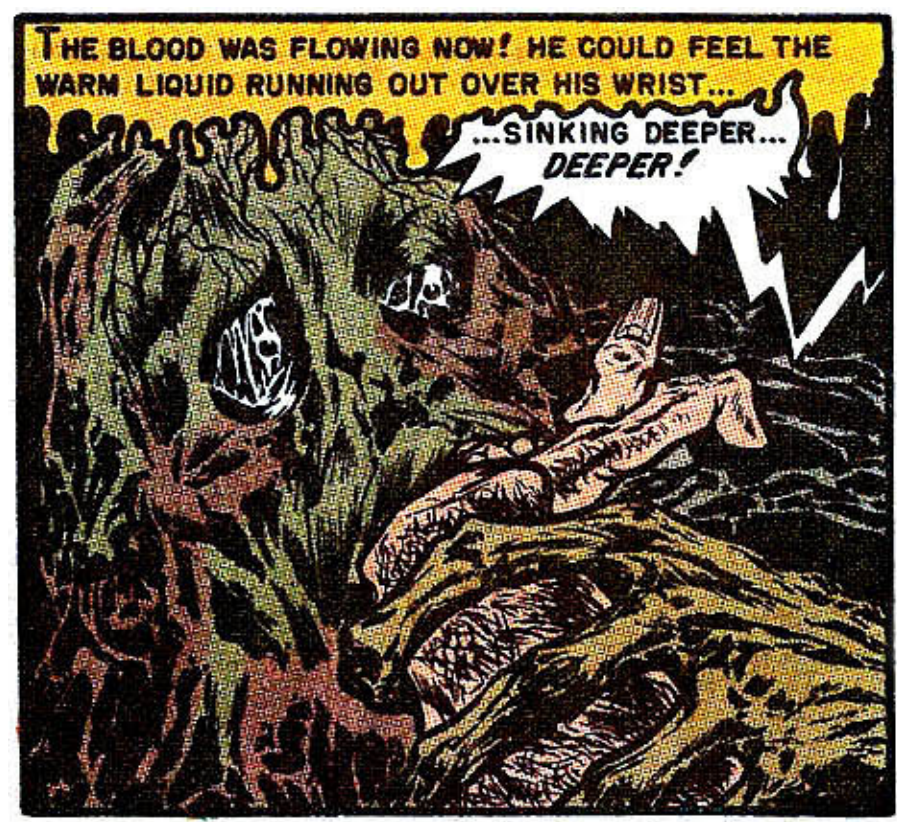

Figure 34: “A Biting Finish,” The Haunt of Fear \#5 (New York: E.C. Comics, 1954).

In his collected volume, The Horror! The Horror!: Comic Books the Government

Didn't Want You to Read, Jim Trombetta writes:

[E.C.] comics were genuinely and brazenly subversive in a way that might not even be possible today. In an era that held its values dearly, no dearly held value survived exposure to the horror comic universe: not the "happy ending," not family, not science, and not the law. This world seems not merely meaningless in an existential sense-godlessness would be a relief-but also actively malicious. ${ }^{413}$

E.C. horror comics included many monstrous creatures that have since become

common horror narrative tropes. Witches, the undead, possession, and various types of occultism regularly graced the pages of various E.C. horror titles. Many of the creatures and narratives featured in these books challenged entrenched notions of selfhood and identity, because the monstrous antagonists of horror narratives often occupy conceptual spaces between or across culturally accepted categories. The violation of these categories is part of what makes the monsters threatening. Monsters often do not fit into culturally constructed conceptual frameworks and they thereby challenge notions about how the

${ }^{413}$ Jim Trombetta, The Horror! The Horror!: Comic Books the Government Didn't Want You to Read (New York: Abrams ComicArts, 2010): 31. 
world fundamentally operates and what type of entities might exist within it. One such conceptual framework is that of selfhood.

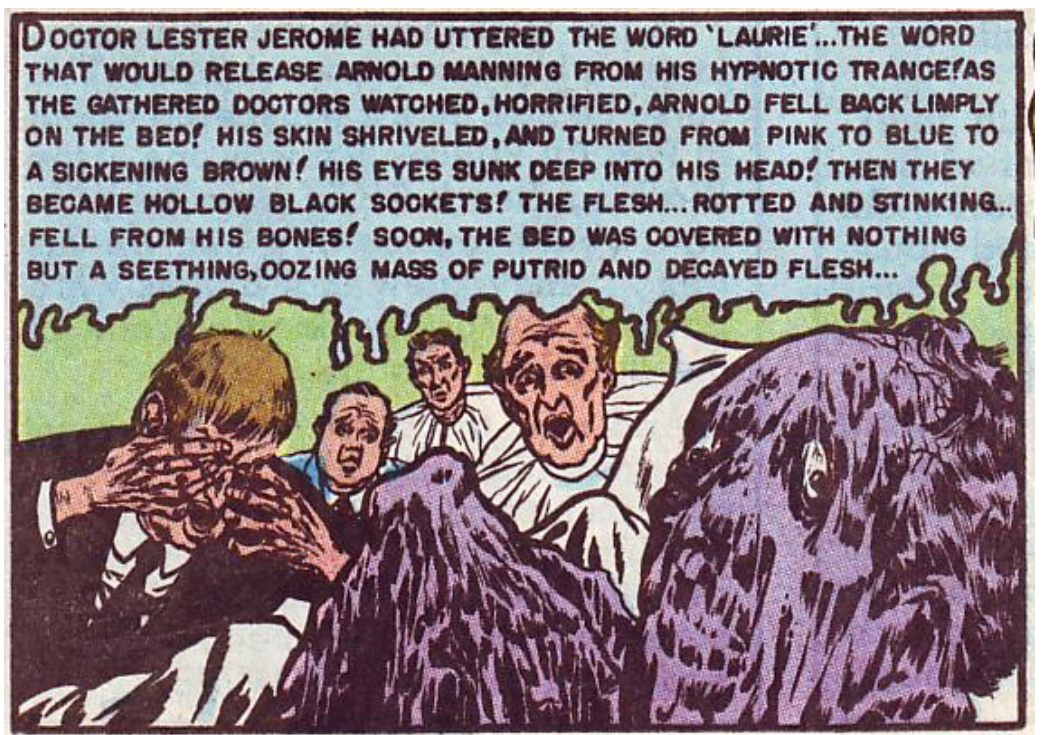

Figure 35: “The Living Death!” Tales From the Crypt \#24 (New York: E.C. Comics, 1951).

Monsters may undermine the distinction between self and not-self. Noël Carroll

writes:

Many monsters of the horror genre are interstitial and/or contradictory in terms of being both living and dead [and] near relatives to these are monstrous entities that conflate the animate and inanimate. [...] Thus, monsters are not only physically threatening; they are cognitively threatening [...] For such monsters are in a certain sense challenges to the foundations of a culture's way of thinking.",414

Monsters, then, narratively undermine culturally accepted or presumed conceptual boundaries and are therefore potentially culturally subversive by their very existence in the narrative.

Through their undermining of conceptual boundaries, many of the monsters in horror comics narratively challenge the common distinction between self and non-self.

E.C. horror comics oftentimes blurred the lines between living and dead characters, and thereby subverted the entrenched view that selves are enduring and essential entities.

${ }^{414}$ Noël Carrol, The Philosophy of Horror: Or, Paradoxes of the Heart (New York: Routledge, 1990): 32, 34. 
Many characters are represented as decomposing at unusually quick rates, going from an animate person to a pile of viscera (either animate or inanimate) in mere moments (Figures 34 and 35).

This quick and abnormal dissolution of the physical boundaries between a particular self and its environment implicitly challenges the notion of a self as a stable and enduring entity. With the depiction of "monsters [that] are vaporous or gelatinous," these comics attribute a horrific "formlessness" to the selves of the monsters, which is counter to the entrenched Western notion of a stable and unified selfhood. ${ }^{415}$ While the inclusion of these types of monsters does not explicitly endorse a narrative reading of selfhood, the fact that the stories are presented in comics can imply such a reading, for all the reasons discussed in the previous chapter.

But it is not only the physical boundaries of selfhood that are artistically subverted by the narratives of horror comics. The psychological continuity and unity of selfhood is also regularly called into question. Consider Basil Wolverton's story entitled "Nightmare World," which originally appeared in 1952 in Weird Tales of the Future. "Nightmare World," like many stories in horror comics from the time, addresses the protagonist in the second-person perspective. This seems to be intended to encourage reader identification with the protagonist. In this case, Herman Lasher is a scientist who has "tried to summon the powers of the subconscious mind by the use of chemicals [in order to] delve deeper into those hidden recesses of [his] mind." ${ }^{416}$ Herman ingests his mysterious chemicals and finds himself inside his own subconscious mind.

\footnotetext{
${ }^{415}$ Carrol, 33.

${ }^{416}$ Basil Wolverton, "Nightmare World," Weird Tales of the Future \#3 (New York: Key Publications, 1952).
} 


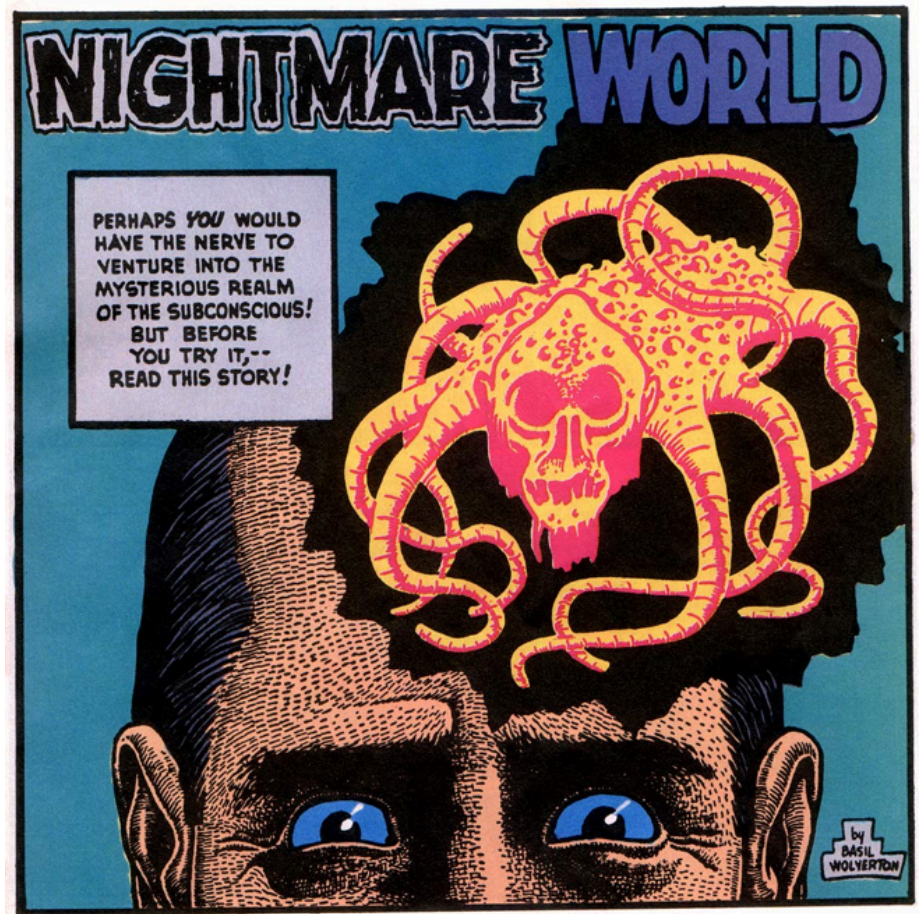

Figure 36: Basil Wolverton, "Nightmare World,"

Weird Tales of the Future \#3 (New York: Key Publications, 1952).

Over the course of the story (four pages in length), it becomes clear that Herman Lasher's subconscious consists of an "alien landscape" filled with strange humanoid monsters that glide similarly to flying squirrels (Figure 37). One of these monsters performs a brain transplant, turning Herman into a similar creature. The story concludes when Herman awakens to find that he has become one of these squirrel monsters in his waking life as well. The moral of the story seems to be: be careful exploring your own subconscious mind. 


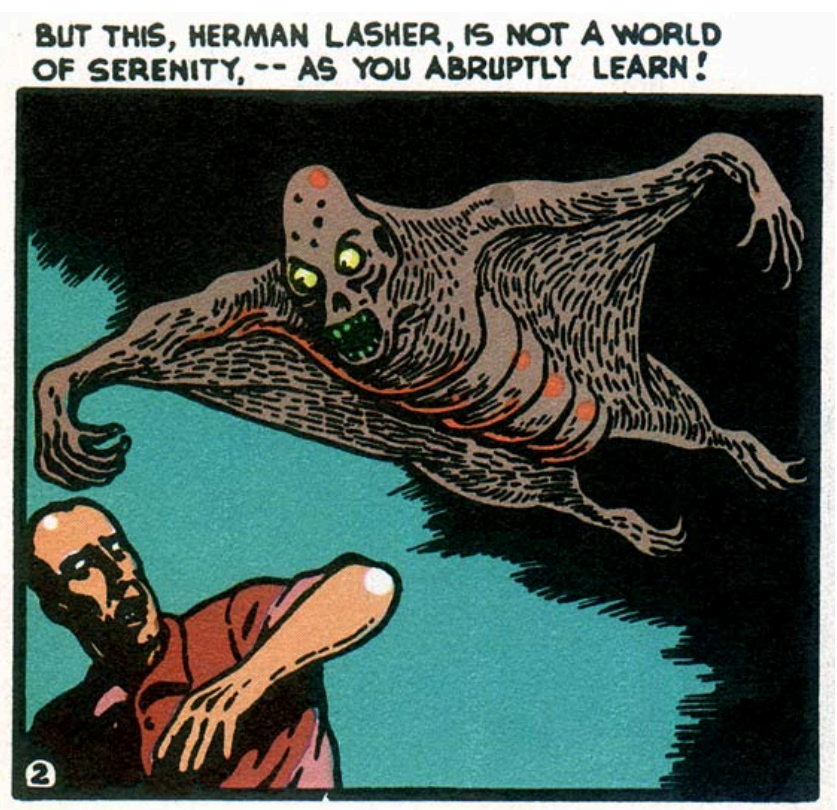

Figure 37: Wolverton, "Nightmare World," Weird Tales of the Future \#3.

The key point about "Nightmare World" is the story's implicit undermining of the notion of a unified psychological selfhood for the character of Herman Lasher. When Herman ingests the chemicals, he "revives" into the world of his own "inner mind.",417 Monsters like the one depicted in Figure 37 inhabit the "alien landscape" of Herman's mind. Finally, when the brain transplant is performed on Herman, he is described as experiencing only "semi-consciousness" even though he already inhabits the world of his inner mind (Figure 38).

These narrative turns imply that Herman's ordinary waking consciousness contains an "alien landscape" with various geographical features, multiple subconscious monsters with volitional abilities that are potentially contrary to Herman's, and a further possible level of non-consciousness that Herman is able to experience within his own subconscious. Herman Lasher, according to "Nightmare World," does not consist of a unified and essential self. Rather, he is multiple, divisible, and capable of containing

${ }^{417}$ Wolverton, "Nightmare World," Weird Tales of the Future \#3. 
various levels of narrativizable components. We see, in examples from these horror comics, challenges to the culturally entrenched notions of both the physical and psychological boundaries between self and non-self.

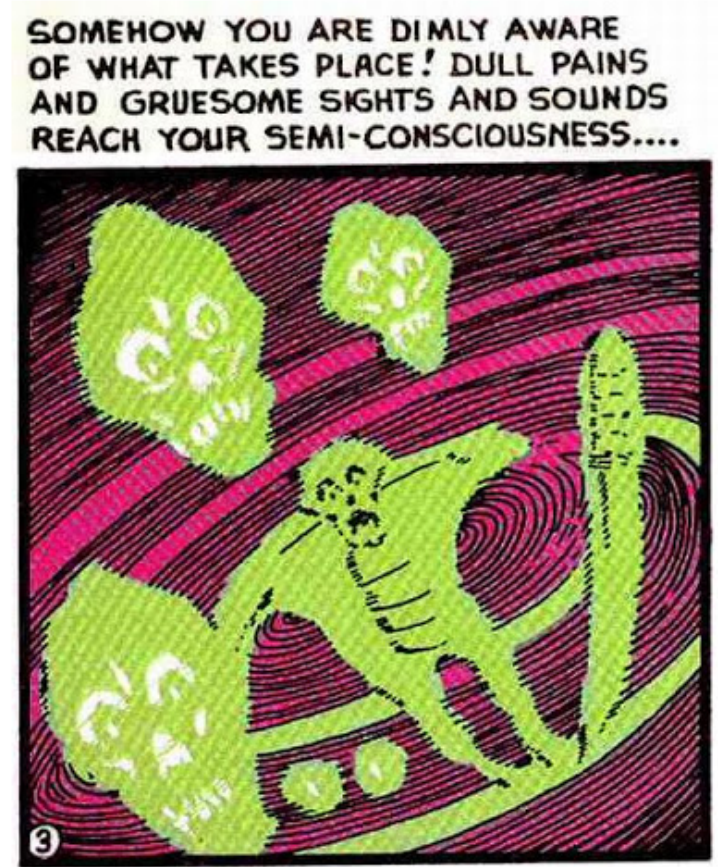

Figure 38: Wolverton, "Nightmare World," Weird Tales of the Future \#3.

The previous examples show clearly the potential threat to Western normative conceptions of selfhood that horror comics made available to their readers, and make intelligible a potential undercurrent of the anti-comics panic of the mid-Twentieth century. It is clear that the attempted (and ultimately, self-imposed) censorship of the comics industry was not simply about representations of crime and criminality. This censorship was also about representations of immoral and subversive values more broadly. The 1948 version of the Comics Magazine Association of America's comics code instructed that, "crime should not be presented in such a way as to throw sympathy against law and justice or to inspire others with the desire for imitation." ${ }^{418}$ When the

${ }^{418}$ Nyberg, Seal of Approval, 165. 
code was updated in 1954, it included the direction that "in every instance good shall triumph over evil and the criminal [shall be] punished for his misdeeds."419 "In fact, the Code $[\ldots]$ would not give its stamp of approval on comics with supernatural content at all, and the words 'terror' and 'horror' were banned outright from titles." ${ }^{420}$ Here, we can see the focus on a moral and arguably religiously normative focus in the anti-comics movement.

Many of the values that these comics subverted were social and cultural. Horror and crime comics often explicitly undermined trust in social institutions, such as the police and government. However, part of what comics threatened were those values that were perceived as being relevant to normative American religiosity. As I have argued, the common Western understanding of the self is intimately conceptually related to the religious notion of the soul. Therefore, comics, insofar as they implicitly present views of selfhood that challenge this entrenched notion, are subversive in relation to normative Western religiosities. It is unsurprising, then, that we see explicit narrative representations of subversive religiously resonant traditions of thought in the work and lives of some prominent comics creators.

Occultist Views of Selfhood, Reader Consciousness, and Comics

The self, as I have argued, is presented as implicitly narrative in nature by the ways in which readers engage with comics and this illuminates the tendency for comics to present narratives in which traditional Western understandings of selfhood are subverted in various ways. Superhero stories frequently deal in dual-identities and

\footnotetext{
419 Nyberg, 167.

${ }^{420}$ Winslade, "Enrolling in the 'Hidden School,"” 199-200.
} 
various supernatural elements. Often, science fiction comics highlight the transience and cosmic insignificance of individual selves, as well as threats to the self that derive from the reaches of outer space. Horror comics utilize monstrous creatures to undermine both the physical and psychological barriers between self and non-self.

Many of these comics involve esoteric and occultist symbolism and narrative elements. Occultism and esotericism researcher Jason Winslade writes, "the development of comic books in the twentieth century closely paralleled the occult explosions of the 1960s, with its countercultural explorations of alternative spirituality and psychedelia, and the 1970s, which saw the emergence of witchcraft and magic into popular consciousness. ${ }^{\text {"421 }}$ Comics' representations of characters that implicitly and narratively undermine the entrenched Western notion of selfhood can be found in more explicit forms as well. Some such representations and narratives might even enable readers to experience and conceptualize their own selves in non-normative ways.

Comics authors sometimes utilize their works to process and examine their own anomalous experiences or conceptualizations of selfhood. Consider Alan Moore, who is one of the most celebrated and accomplished writers to ever work in the comics medium. His work has found both critical and popular success and his graphic novel Watchmen deconstructed the archetype of the superhero in a way that made "all superhero comics from then on [be considered as] either pre- or post-Watchmen." ${ }^{, 422}$ But it was Moore's post-Watchmen work that is most relevant with regard to the current discussion of occultism and esotericism.

\footnotetext{
${ }^{421}$ Winslade, 199.

${ }^{422}$ Wolk, Reading Comics, 244.
} 
While for a time Moore understood himself as a "rationalist and an atheist," 423 in his later career he has become heavily interested in esoteric and occultist systems of thought. He even declared himself a ceremonial magician in 1993, on the night of his fortieth birthday. ${ }^{424}$ In 1994, Moore reported having "experienced the occult presence of what seemed to be the second-century Roman snake god Glycon, who, Moore tells us, was believed to be the reincarnation of Asclepius, the god of healing." ${ }^{425}$ This encounter led Moore to believe that most people's everyday experiences of themselves were incomplete at best. In an interview, Moore recounted:

At least part of this experience seemed to be completely outside of Time. There was a perception that all of Time was happening at once. Linear time was a purely a construction of the conscious mind, and in fact Time is much more the way that people like Stephen Hawking seem to describe it, where Space-Time is almost like some big football, and you've got the Big Bang at one end of it and the Big Crunch at the other, but all of the moments are all existing at once, in this huge hole at the moment. It's only our consciousness that's moving through it, from A to B to C to D. In fact, the whole alphabet's there right from the start. So there was this perception of being outside of Time. From that perspective, it was possible to see that all of Time was in fact happening at once. ${ }^{426}$

Moore's strange experience of himself stretched throughout time very much mirrors the way that comics represent narrative time, in concurrently existing static pieces of visual art. Moore represented and explored his anomalous experiences and occultist beliefs through his self-published series Promethea.

Promethea is ostensibly a superhero story, but quickly evolves into a narrative thesis on Moore's own brand of esoteric occultism. The series follows the adventures of college student Sophie Bangs, living in a hyper-realistic version of New York in the year

${ }^{423}$ Krypal, Mutants and Mystics, 15.

${ }^{424}$ Sam Proctor, “Alan Moore: The Art of Magic," Pagan Dawn, February 12, 2016, http://www.pagandawnmag.org/alan-moore-the-art-of-magic.

${ }^{425}$ Krypal, Mutants and Mystics, 16.

${ }^{426}$ Babcock, "Magic is Afoot," Arthur, https://arthurmag.com/2007/05/10/1815. 
1999. When researching mythology in order to write a college term paper, Sophie

unwittingly comes into contact with the demi-goddess Promethea: a living story who has been manifested in various women throughout history. Promethea was originally a young girl living in fifth-century Alexandria who, after almost being murdered by a group of Christians as a child, escaped into the realm of imagination (known as the "Immateria") and became the first person to embody this demi-goddess. Over the course of the series' 32-issue run, Sophie Bangs becomes the latest incarnation of Promethea and comes to understand her purpose as ushering in a utopian apocalypse.

Promethea narratively explores Moore's personal occultist view of the world, which is heavily influenced by various Western esoteric traditions and practices, including hermetic Kabbalah. ${ }^{427}$ Moore's use of the character of Promethea has implications for the view of the nature of selfhood implied (and sometimes explicitly stated) by the narrative. During her adventures, "Sophie comes to find that each Promethea was made manifest in reality when an author or artist conjured her through an act of creating art; composing a story of Promethea made one into Promethea." ${ }^{428}$ This narrative device demonstrates Moore's belief that stories are "powerful, yet adaptable, entities that cooperate to create our notion of reality. ${ }^{, 429}$ In other words, the narratives that people construct serve to constitute those very people. Moore's occultist view of the power of stories clearly parallels the narrative view of selfhood discussed in the previous chapter.

${ }^{427}$ Lewis, American Comics, Literary Theory, and Religion, 118. ${ }^{428}$ Lewis, 118.

${ }^{429}$ Jackson Aryes, "Promethea," in The Encyclopedia of Comic Books and Graphic Novels Volume 2, ed. M. Keith Booker (Denver, CO: Greenwood, 2010): 489. 


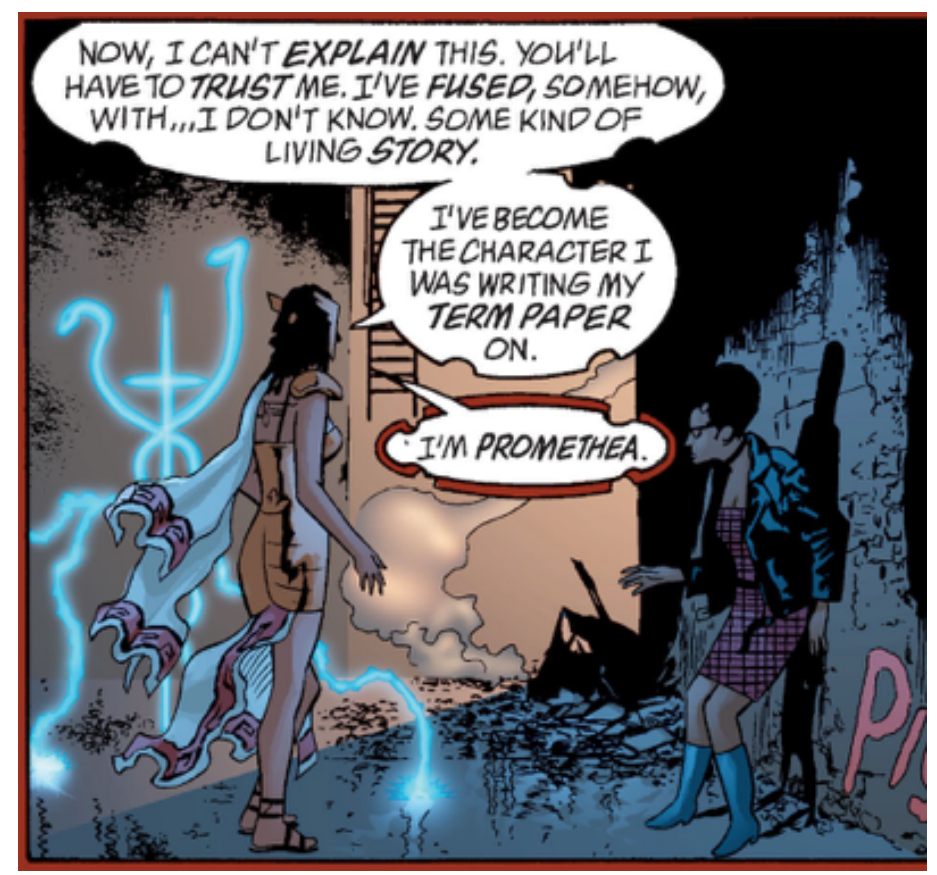

Figure 39: Alan Moore and J.H. Williams III, Promethea: Book One (La Jolla, CA: America's Best Comics, 2000).

Sophie becomes the story that she constructs. Because the story is about

Promethea, Sophie becomes Promethea. When Sophie's Promethea ushers in the apocalypse at the end of the series, "'the world' that is destroyed is not our physical world, but rather our illusory constructions of reality [...] For Moore, as for many of the Romantics, imagination is a divine attribute and a way to participate in the ongoing creation of the universe. $" 430$ The world that ends is the result of destructive and harmful interpretations, rather than being the physical world upon which those interpretations are based. On this occultist view, the physical world is only part of the world, because humans' concepts, language, and interpretations also constitute humans' world and themselves. The narrative view of selfhood similarly privileges the creative and interpretive acts of humans in the construction of their self-narratives.

${ }^{430}$ Christine Hoff Kraemer and J. Lawton Winslade, "'The Magic Circus of the Mind": Alan Moore's Promethea and the Transformation of Consciousness through Comics," in Graven Images: Religion in Comic Books and Graphic Novels, eds. A. David Lewis and Christine Hoff Kraemer (New York: Continuum Intl. Publishing Group, 2010): 276. 
Because of the perceived importance of language and interpretation, occultist and esoteric traditions often utilize art, text, and symbolism in the construction and interpretation of meaning, and as a way to influence reality.

In Western esotericism, literature (in which we include images and numbers) is generally conceived as a means of transmuting consciousness. The words and images of Western esoteric traditions are by no means diversions, or mere entertainment, but rather, although they may contain an element of play, are in fact essentially gnostic: that is, they are charged with visionary or spiritual experiences, and are meant to lead their reader towards those experiences. ${ }^{431}$

In the latter half of Promethea, "the series abandoned any pretense of being a traditional superhero book and took its heroine on a journey through each of the spheres of the kabbalistic Tree of Life, the Hebrew mystical system appropriated by Western occultists. ${ }^{, 432}$ Moore utilized the ostensibly superhero comics series to expose his readers to his own esoteric cosmology and experiences. Moore even gives a cameo to the most famous ceremonial magician of the last century, when Sophie encounters Aleister Crowley in a space between two of the spheres of the Tree of Life (sephirah). The series is deeply rooted in the symbology and concepts of occultist and esoteric movements of the late Nineteenth and Twentieth centuries, but it is potentially more than only a narrative featuring obscure elements from the history of occultism.

Promethea has the potential to serve as induction into occultist practices and experiences for its readers. Rather than only functioning to explain Moore's occultist Kabbalistic cosmology and understanding of narrativized selfhood in conceptual terms, Promethea provides readers with the opportunity to engage with their understanding of their own selfhood on Moore's terms (that is, as narrative in form). The final issue of the

${ }^{431}$ Arthur Verslius, "Western Esotericism and Consciousness," Journal of Consciousness Studies 7, no. 6 (2000): 22.

${ }^{432}$ Kraemer and Winslade, “"The Magic Circus of the Mind,”" 274-5. 
series completely abandons the narrative format and simply consists of the character of Promethea addressing readers directly, providing them with a conceptual overview of Moore's esoteric cosmology. In this final issue, Promethea advocates the idea that consciousness, imagination, and language co-create reality, and therefore, human's linguistic and imaginative acts have the potential to shape their world. The direct address to readers by the character of Promethea serves to break the fourth wall and invite readers to think of their own lives in these esoteric terms.

It is clear that Moore intended the series to function as an introduction to his worldview, but also that Promethea is crafted to shape readers' consciousnesses in ways that Moore would call "magical." The potential for reader engagement in the way the comic is crafted, combined with the common view of esoteric traditions that "the words and images [of a text] can evoke in the reader the seeds of the experiences visible through the work," ${ }^{433}$ make it clear that Moore is attempting to shift reader consciousness, and not only to create a cultural artifact intended for entertainment. The final issue consists of thirty-two pages featuring:

non-ordered panels consisting of overlapping line drawings brushed with psychedelic pastels and sprinkled with stars and ankhs [...] Readers are encouraged to remove the pages from the final issue and juxtapose them in whatever way they choose. However, when put in a certain order, the pages form poster-sized paintings of Promethea's face, which emerges from what otherwise looks like a jumble of random images and text. ${ }^{434}$

The potential for the reader to physically dismantle and rearrange the pages of the final issue increases the potential depth of reader engagement and highlights, even more than a standard comic book, the role of the reader in co-constructing the meaning of the work.

${ }^{433}$ Verslius, "Western Esotericism and Consciousness," 27.

${ }^{434}$ Kraemer and Winslade, "“The Magic Circus of the Mind,", 285. 
Moore's point, made visually, is that humans' ability to interpret and conceptualize the world creates our selves and our world.

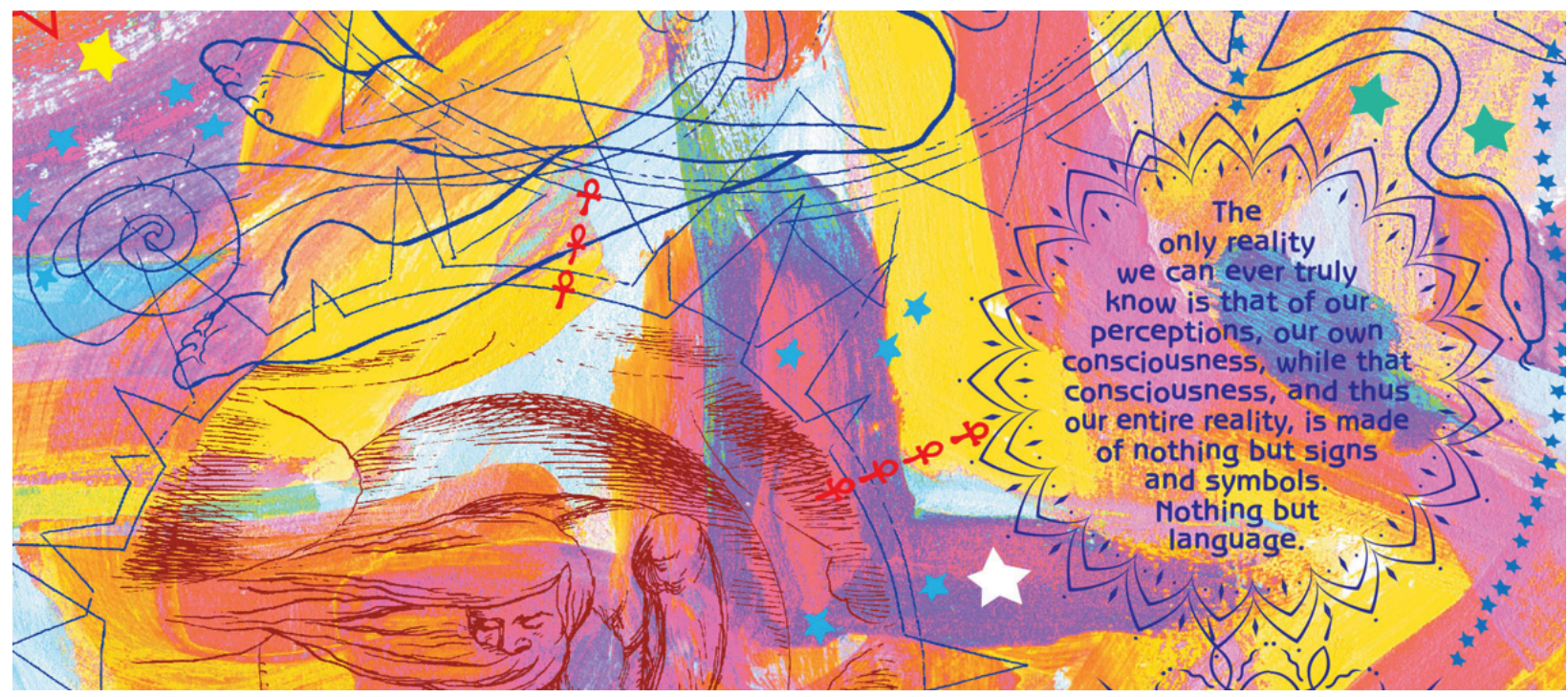

Figure 40: Alan Moore and J.H. Williams III, Promethea: Book Five (La Jolla, CA: America's Best Comics, 2000).

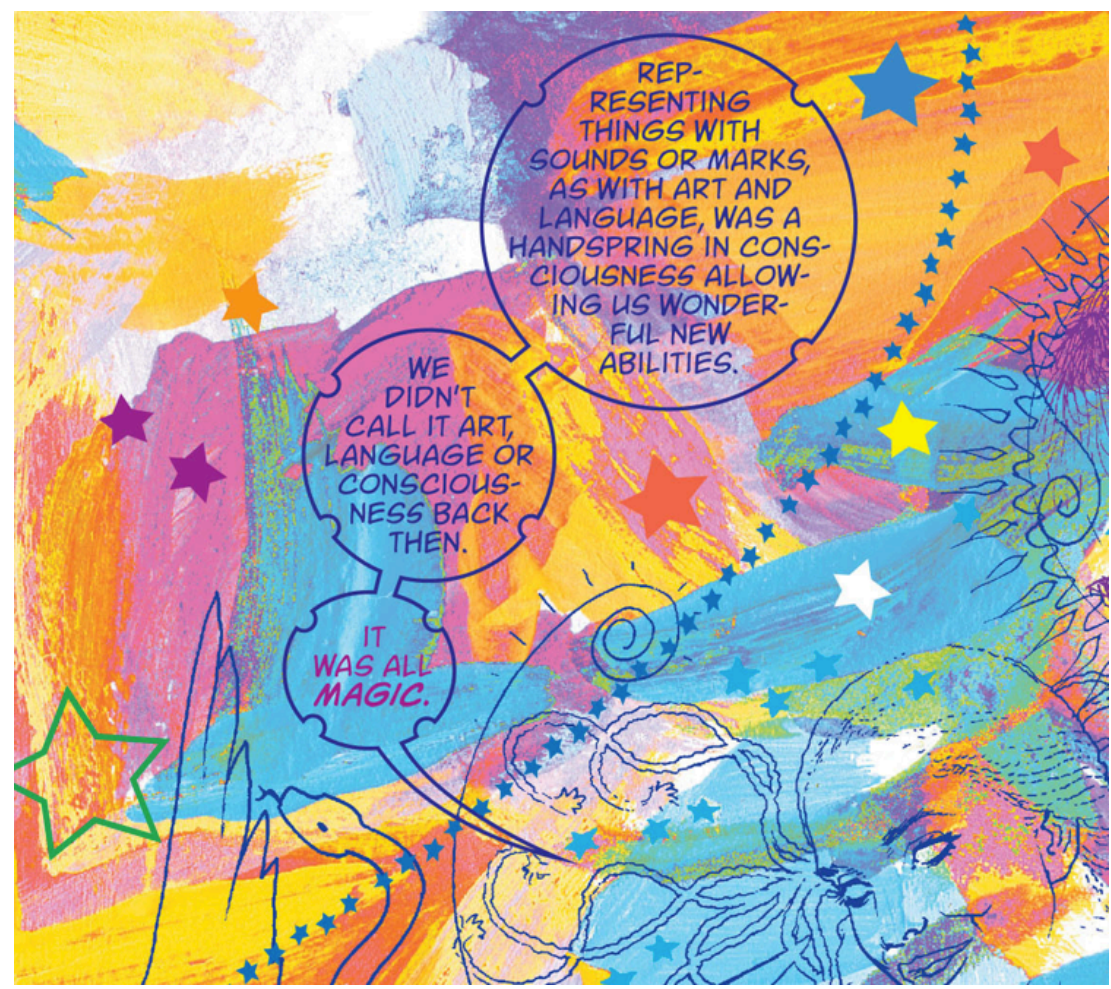

Figure 41: Moore and Williams III, Promethea: Book Five.

The smeared watercolor-style backdrop of the final images of Promethea suggests an apparently chaotic background contrasted against the blue lines that constitute the text 
and more clearly representational images. This contrast visually embodies the (in Moore's sense) "magical" notion that distinctions in the world come to be realized when humans purposefully enact lines of division (conceptual, evaluative, etc.) onto seemingly random and disordered elements. The distinction between the figurative foreground and the seemingly abstract background in the final issue of Promethea, then, parallels the contrast between an unordered chaotic world and the symbolically and linguistically ordered meaning that humans enact upon it. For our purposes, the most relevant instance of this type of "magical" meaning projection is the development of the narrative of selfhood that allows individuals to understand and predict themselves. The physical organism with which the self is associated is the somewhat chaotic and disunified background onto which individuals (and their communities) project meaningful narration through symbols and language. Moore would call this project, "magic."

This occultist view certainly goes much farther than philosophical models of narrative selfhood, by privileging the role of human consciousness, imagination, and language in the determining of reality broadly, and not only our self-narratives. However, the parallels are clear. On both views, our selves are not determinate entities that exist alongside other things in the material universe. Rather, our selves are the result of creative, imaginative, and interpretive representations and narrativizing of various physical events and systems. Occultists like Moore label this ability to narrativize one's selfhood "magic," while philosophers like Marya Schechtman call it the development of an "autobiographical narrative which acts as the lens through which we experience the 
world. ${ }^{, 435}$ In both cases, selves are interpreted narrative constructions, contra the common Western view.

Esoteric and occultist traditions' view that the self is a conventional linguistic construction of human consciousness and the philosophical view that selves consist of narratives that human organisms develop in order to explain, predict, and interpret the behavior of certain physical systems are analogous and converge in the formal features and narrative content of many comics. In Promethea we see a clear example of the cultural-historical and aesthetic relationships between the formal features of comics, the narrative conception of selfhood, and occultist and esoteric traditions. The types of reader engagement required by comics' use of both textual and visual artistic elements make comics incredibly conducive to narratives that explore issues of selfhood and identity, as well as representations of occultist systems of thought.

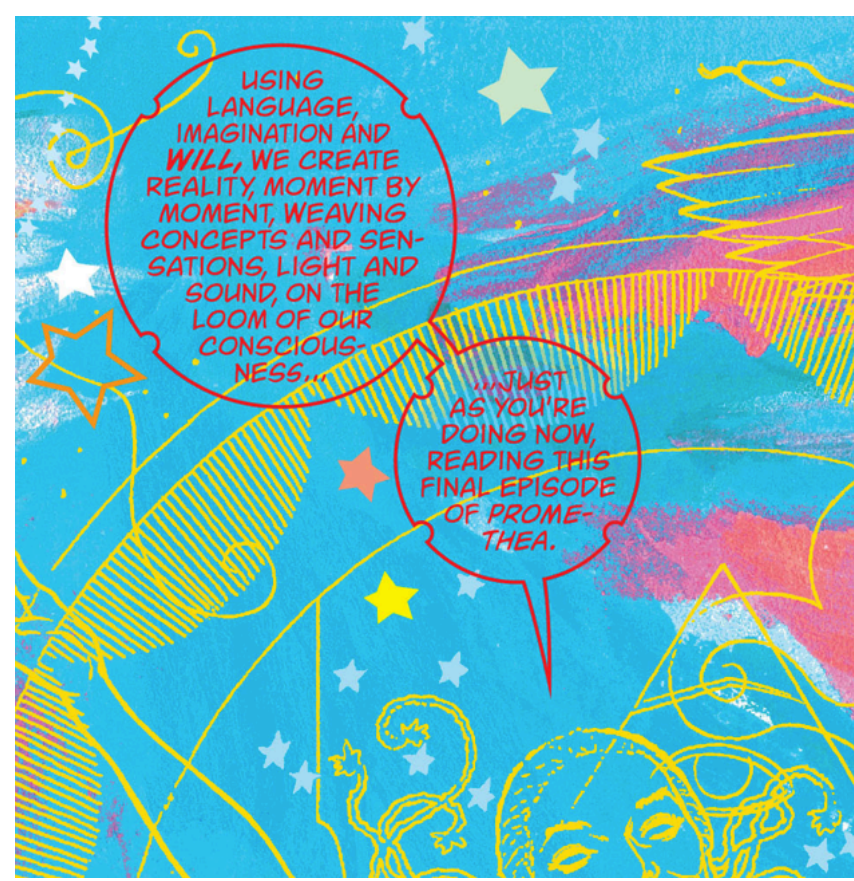

Figure 42: Moore and Williams III, Promethea: Book Five.

${ }^{435}$ Schechtman, Staying Alive, 100. 
Conclusion: Form and Content

Over the course of this work, I have illuminated the relationships between readers' typical engagement with the formal features of comics and the types of content that often appear in American comics. I have contextualized the cultural-historical tendency of comics to traffic in occultist, esoteric, supernatural, subversive, and religiously resonant narrative content in terms of the conception of selfhood implied by the form of comics. Because the comics format typically utilizes both images and text in order to render narratives, "comic art is complex enough to frustrate any attempt at an airtight analytical scheme. ${ }^{, 436}$ Rather than offer a comprehensive framework of all possible engagement with comics, I detailed some forms of engagement with comics that are typical.

Readers' perceptual experiences with the text and sequential static visual artwork of comics necessitates that readers enact the cognitive processes that constitute closure in order to understand comics narratives. Readers typically infer conceptual information regarding the narratives and imagine motion and duration in the storyworld from panel to panel. The cognitive processes that constitute closure allow readers to infer and imagine a coherent storyworld (characters included) across the discreet static images and textual elements. Because of this, readers are actively involved in uncovering and constructing the story and meaning of a comic.

The need for readers to enact closure also results in unique types of emotional affordances. Comics depict characters rather than only textually describing them, which allows for emotional contagion, empathic, and sympathetic reader responses. Artistic and

${ }^{436}$ Charles Hatfield, Alternative Comics: An Emerging Literature (Jackson, MS: University Press of Mississippi, 2005), 66. 
design elements of comics provide unique opportunities for emotional engagement by influencing readers" "non-cognitive" affective appraisals. ${ }^{437}$ Hermeneutic images, for example, visually represent emotionally salient perspectives on the events or characters in the storyworld, rather than mimetically representing objects or characters. Readers respond affectively to the artistic tone of the images and this can influence their expectations regarding narrative content before they have registered the conceptual content of the images.

In the context of American cultural history, the multiple sequential static representations of characters that typically appear in comics have potentially subversive implications with regard to the concept of selfhood. The entrenched traditional Western understanding of the self, which is indebted to the Western religious concept of the "soul," is that the self is essential, continuous, and unified. However, the typical formal features of comics implicitly undermine this model. Multiple static images of a single character that co-exist on a typical comics page (and therefore concurrently in the reader's visual field) highlight the role of reader engagement in the construction of the coherence of the character's self. The self of a character is not simply presented by the comic, it is constructed, interpreted, and narrativized in the mind of the reader.

Multiple and potentially divergent images of one character being seen at once across multiple moments of narrative time require the reader to imagine and infer coherence (that is, enact closure) across the distinct representations on the page. Additionally, artistic and style elements of comics may visually portray amorphous or porous boundaries between a character and her environment (that is, between self and

${ }^{437}$ Robinson, Deeper Than Reason, 55 
non-self). Readers' active narrativizing of the selves of comics characters is analogous to the notion of actual selves put forth by philosophers who affirm a narrative view of selfhood.

These philosophers claim that actual selves are the result of active narrativizing by individuals and communities. Selves, in this view, are individually and socially constructed "abstractums," 438 interpretations of the actions and motivations of particular organisms. Therefore, we can distinguish between a self and the physical organism with which that self is associated. Similarly, we can distinguish between the self of a comics character (that is, the inferred and imagined coherent narrativized character) and the images on the comics page that depict the character. The comic does not (absent active interpretations) deliver a singular unified comics character. Similarly, the physical world does not (absent active interpretations) deliver the narratively unified selves of individuals.

Finally, I have explored how this confluence between narrative conceptions of selfhood and readers' typical cognitive and emotional engagement with the formal features of comics can help make sense of the cultural history of comics since their rise to prominence in the U.S. over the past century. American comics often traffic in supernatural, subversive, occultist, esoteric, and religiously resonant narrative content. For example, "the pillaging by superhero comic writers of mythology, folklore, and biblical and classical literature for stories is well documented." ${ }^{439}$ The tendency for

\footnotetext{
${ }^{438}$ Dennett, "The Self as a Center of Narrative Gravity," http://cogprints.org/266/1/selfctr.htm.

${ }^{439}$ Lucy Wright, "Shamans vs (Super)heroes," in Super/Heroes: From Hercules to Superman, eds. Wendy Haslem, Angela Ndalianis, and Chris Mackie (Washington, DC: New Academia Publishing, 2007): 132.
} 
comics to traffic in these themes is wholly intelligible in light of the potentially subversive implications of selfhood inherent in the typical formal features of comics.

The cultural history of comics in America dovetails and overlaps with esoteric and occultist traditions, both influencing them and being influenced by them. Some comics creators have even infused their work with concepts and experiences from their own esoteric practices. As Jeffrey Kripal writes, "there is no way to disentangle the very public pop-cultural products from the very private paranormal experiences [of these authors]. ${ }^{440}$ Superhero, science fiction, and horror comics all undermine the common Western view of selfhood and have been at times perceived as subversive by "parents, politicians, and psychologists. ${ }^{, 441}$ Esoteric and occultist traditions often utilize visual and textual art in their practices and comics, in this sense, are a natural ally.

Esotericism scholar Arthur Versluis writes that, "one could make the case that esoteric traditions were (and continue to be) transmitted in the West primarily via literature and art [...] In truth, we hardly should be surprised when literary works turn out to have hidden esoteric dimensions and many layers of meaning.. ${ }^{, 42}$ It is unsurprising, then, that some comics series not only imply esoteric or occultist views of selfhood (such as, Doctor Occult and Weird Science), but many explicitly affirm such views, inviting the reader to participate in the construction of their own self-interpretation and selfnarrativization (such as, The Invisibles and Promethea). Alan Moore argues, "if you start looking beyond the confines of self-declared magicians, then it becomes increasingly difficult to find an artist who wasn't in some way inspired either by an occult

\footnotetext{
${ }^{440}$ Kripal, Mutants and Mystics, 2.

${ }^{441}$ Karin Kukkonen, Studying Comics and Graphic Novels (Malden, MA: Wiley Blackwell, 2013): 110.

${ }^{442}$ Versluis, Magic and Mysticism, 154.
} 
organization or an occult school of thought or by some personal vision." ${ }^{443}$ Comics and occult esoteric traditions are historically intertwined with good reason.

This present work clearly is far from comprehensive. My hope is that this work opens many possible areas for future research. For example, future works might explore how particular intersectional identity elements influence the conceptions of selfhood put forth by various comics. How do the gender, race, and class of particular characters interact with the standard implications of comics for selfhood to create new narrative, formal, or philosophical possibilities? Additionally, future work may explore genres of comics that are beyond the scope of the current project, such as non-fiction, autobiographical, or memoir comics.

While she discusses representations of subjectivity and selfhood in relation to womanhood and issues of gender, comics scholar Hillary Chute's analysis of the possibilities of the comics medium highlights the potential relevance of the present work to memoir and autobiographical comics. The intersection of these two streams of research could open up exciting new questions and opportunities for cultural and philosophical investigation. Chute writes:

Unsettling fixed subjectivity, [memoir comics] present life narratives with doubled narration that visually and verbally represents the self, often in conflicting registers and different temporalities. [Comics authors often] use the inbuilt duality of the form-its word and image cross-discursivity-to stage dialogues among versions of self, underscoring the importance of an ongoing, unclosed project of self-representation and self-narration [...] Through its hybrid and spatial form, comics lends itself to expressing stories, especially narratives of development, that present and underscore hybrid subjectivities. ${ }^{444}$

\footnotetext{
${ }^{443}$ Babcock, "Magic is Afoot," https://arthurmag.com/2007/05/10/1815.

${ }^{444}$ Hillary Chute, Graphic Women: Life Narrative and Contemporary Comics (New York: Columbia University Press, 2010): 5.
} 
Autobiographical and memoir comics are, quite literally, instances of self-narration, through the use of image and text. These genres of comics, as Chute articulates, allow their authors (and readers) to understand story telling about the self to be an open-ended endeavor. Self-narratives are susceptible to multiple and distinct structures of meaning and various interpretive frameworks through which individuals and their communities can construct differential "versions of self." ${ }^{445}$ Again, this project of utilizing symbols (image and text) to shape ourselves would be called, by many occultists, "magic." There are likely other possibilities for future research that I have not mentioned or even considered.

In large part, this interdisciplinary project has been an attempt to connect the form with the content of comics across various levels of analytical scope (from the finegrained perceptual to the broadly cultural). Ultimately, I have argued that the relationship between typical narrative themes in comics and comics themselves are not only the result of historical accidents. Rather, there is an intelligible connection between the way we read comics and the types of things that comics are often about.

Many of the genres that are most popularly associated with the comics form implicitly undermine the common Western view of selfhood. I have shown that this undermining occurs not only through narrative content, but also through the types of experiences that readers have when engaging with the form of comics itself. This link between form and content makes an occultist comic like Promethea entirely comprehensible. Promethea utilizes the implications for selfhood of the typical formal features of comics to, as Christine Hoff Kraemer puts it, "produce comic books that are

${ }^{445}$ Chute, 5 . 
expressions of spiritual experiences, and which in turn provide the reader opportunities for similar spiritual experiences. ${ }^{.446}$ Comics, within the American context, are potentially culturally and religiously subversive regardless of the particular stories they tell because the form of comics undermines traditional notions of selfhood, as I have shown. Comics bring us fantastical, weird, and subversive stories in particularly effective ways because of how we read and view them. The reading experiences that comics afford us shape the types of stories and themes that are most effectively communicated to us by comics. Therefore, comics can have fascinating implications for how we understand and experience ourselves.

${ }^{446}$ Christine Hoff Kraemer, "Promethea: Comics as Spiritual Tool," 2003, http://www.christinehoffkraemer.com/promethea-exp.html. 


\section{REFERENCES}

“4 ${ }^{\text {th }}$ Wall Awareness.” SuperpowerWiki. Accessed February 19, 2017. http://powerlisting.wikia.com/wiki/4th_Wall_Awareness.

“A Biting Finish.” The Haunt of Fear \#5. New York: E.C. Comics, 1954.

Aryes, Jackson. "Promethea." In The Encyclopedia of Comic Books and Graphic Novels Volume 2, edited by M. Keith Booker, 487-489. Denver, CO: Greenwood, 2010.

Babcock, Jay. "Magic is Afoot: A Conversation with Alan Moore about the Arts and the Occult." Arthur. May 2003. https://arthurmag.com/2007/05/10/1815.

Baetens, Jan and Hugo Frey. The Graphic Novel: An Introduction. New York: Cambridge University Press, 2015.

Barresi, John and Raymond Martin. "History as Prologue: Western Theories of the Self." In The Oxford Handbook of the Self, edited by Shaun Gallagher, 33-56. New York: Oxford University Press, 2011.

Bateman, John and Janina Wildfeuer. "Defining Units of Analysis for the Systematic Analysis of Comics: A Discourse-based Approach." Studies in Comics 5, no. 2 (2014): 373-403.

Beaty, Bart. Fredric Wertham and the Critique of Mass Culture. Jackson, MS: University Press of Mississippi, 2005.

Begley, Chris. "Grant Morrison: Batman kills Joker in 'The Killing Joke."” Batman News. August $16^{\text {th }}$, 2013. http://batman-news.com/2013/08/16/grant-morrisonbatman-kills-joker-in-the-killing-joke/.

Bendis, Brian Michael and Avon Michael Oeming. Powers: Forever. New York: Icon Comics, 2004.

Benton, Mike. Science Fiction Comics: The Illustrated History. Dallas, TX: Taylor Publishing Company, 1992.

Blakney, Raymond, trans. Meister Eckhart: A Modern Translation. New York: Harper \& Row, 1941. 
Bloom, Paul. Descartes' Baby: How the Science of Child Development Explains What Makes Us Human. New York: Basic Books, 2004.

---. "Natural-Born Dualists.” Edge. May 11, 2004. https://www.edge.org/conversation/natural-born-dualists.

Bolotin, Arkady. "The Quantum Pigeonhole Principle as a Violation of the Principle of Bivalence.” Quantum Studies: Mathematics and Foundations (2017): 1-6.

Boyd, Brian. “On the Origin of Comics: New York Double-Take.” Evolutionary Review 1 (2010): 97-111.

Brubaker, Ed and Doug Mahnke. Batman: The Man Who Laughs. New York: DC Comics, 2008.

Brubaker, Ed and Sean Phillips. The Fade Out: Act One. Portland, OR: Image Comics, 2015.

Burton, Dan and David Grandy. Magic, Mystery, and Science: The Occult in Western Civilization. Bloomington, IN: Indiana University Press, 2004.

Camp, Elisabeth. “Two Varieties of Literary Imagination: Metaphor, Fiction, and Thought Experiments." Midwest Studies in Philosophy 33, no.1 (2009): 107-130.

Carrier, David. The Aesthetics of Comics. University Park, PA: Penn State University Press, 2000.

Carrol, Noël. The Philosophy of Horror: Or, Paradoxes of the Heart. New York: Routledge, 1990.

---. A Philosophy of Mass Art. Oxford: Clarendon Press, 1998.

---. "On Some Affective Relations Between Audiences and the Characters in Popular Fictions." In Empathy: Philosophical and Psychological Perspectives, edited by Amy Coplan and Peter Goldie, 162-184. New York: Oxford University Press, 2011.

Chute, Hillary. Graphic Women: Life Narrative and Contemporary Comics. New York: Columbia University Press, 2010.

Cioffi, Frank L. "Disturbing Comics: The Disjunction of Word and Image in the Comics of Andrzej Mleczko, Ben Katchor, R. Crumb, and Art Speigelman.” In The Language of Comics: Word and Image, edited by Robin Varnum and Christina T. Gibbons, 97-122. Jackson MS: University Press of Mississippi, 2001. 
Cohn, Neil. The Visual Language of Comics: Introduction to the Structure and Cognition of Sequential Images. New York: Bloomsbury Publishing, 2013.

---. "The Limits of Time and Transitions: Challenges to Theories of Sequential Image Comprehension." Studies in Comics 1, no. 1 (2010): 127-147.

---. "Un-Defining 'Comics': Separating the Cultural from the Structural in 'Comics."' International Journal of Comic Art, 7, no. 2 (2005): 1-11.

Cohn, Neil and Stephen Maher. "The Notion of the Motion: The Neurocognition of Motion Lines in Visual Narratives.” Brain Research 1601 (2015): 73-84.

Conner, Amanda, Jimmy Palmiotti, and Chad Hardin. Harley Quinn Volume 1: Hot in the City. New York: DC Comics, 2014.

Coogan, Peter. "The Definition of the Superhero." In Super/Heroes: From Hercules to Superman, edited by Wendy Haslem, Angela Ndalianis, and Chris Mackie, 21-36. Washington, DC: New Academia Publishing, 2007.

Cook, Roy T. "Why Comics are Not Films: Metacomics and Medium-Specific Conventions." In The Art of Comics: A Philosophical Approach, edited by Aaron Meskin and Roy T. Cook, 165-187. Malden, MA: WileyBlackwell, 2012.

Coplan, Amy. "Empathic Engagement with Narrative Fictions." The Journal of Aesthetics and Art Criticism 62, no. 2 (2004): 141-152.

---. “Catching Characters' Emotions: Emotional Contagion Responses to Narrative Fiction Film.” Film Studies 8, no. 1 (2006): 26-38.

---. "Understanding Empathy: Its Features and Effects." In Empathy: Philosophical and Psychological Perspectives, eds. Amy Coplan and Peter Goldie, 3-18. New York: Oxford University Press, 2011.

Cullen, John. "nonlinear.” Nhoj. September 8, 2016. http://nellucnhoj.com/post/150125629359/nonlinear-tumblr-twitter-facebook.

Culp, Brett. "Interview: Brett Culp, director of the Batman Documentary Legends of the Knight.” Flickering Myth. August 15, 2013. https://www.flickeringmyth.com/2013/08/interview-brett-culp-director-ofbatman.

Currie, Gregory and Ian Ravenscroft. Recreative Minds: Imagination in Philosophy and Psychology. New York: Oxford University Press, 2003.

Damasio, Antonio. "Fundamental Feelings.” Nature, 413 (2001): 781. 
de Sousa, Ronald. "Emotion." The Stanford Encyclopedia of Philosophy (Spring 2014 Edition), edited by Edward N. Zalta. Accessed October 28, 2017. https://plato.stanford.edu/archives/spr2014/entries/emotion/.

Debes, Remy. "Neither Here nor There: The Cognitive Nature of Emotion." Philosophical Studies 146, no. 1 (2009): 1-27.

Dennett, Daniel C. "The Self as a Center of Narrative Gravity." In Self and Consciousness: Multiple Perspectives, edited by Frank Kessel, Pamela Cole and Dale Johnson, 103-115. New York: Psychology Press, 1992. http://cogprints.org/266/1/selfctr.htm.

Deonna, Julien A. and Fabrice Teroni. The Emotions: A Philosophical Introduction. New York: Routledge, 2012.

Dini, Paul and Bruce Timm. “Jolly Ol' St. Nicholas.” In The Batman Adventures Holiday Special \#1, 1-13. New York: DC Comics, 1995.

Djikic, Maja, Keith Oatley, Sara Zoeterman, and Jordan B. Peterson, “On Being Moved by Art: How Reading Fiction Transforms the Self." Creativity Research Journal 21, no. 1 (2009): 24-29.

Duncan, Randy and Matthew J. Smith. The Power of Comics: History, Form \& Culture. New York: Bloomsbury Academic, 2015.

Ekman, Paul. "Basic Emotions.” In Handbook of Cognition and Emotion, edited by Tim Dalgleish and Mick J. Power, 45-60. New York: The Guilford Press, 1999.

Eisner, Will. Comics and Sequential Art: Principles and Practices from the Legendary Cartoonist. New York: W.W. Norton \& Company, 1985.

Flanagan, Owen. The Problem of the Soul: Two Visions of Mind and How to Reconcile Them. New York: Basic Books, 2002.

Freaky Friday. Directed by Gary Nelson. 1976; Walt Disney Pictures, DVD.

Freeberg, David and Vittorio Gallese. "Motion, Emotion and Empathy in Esthetic Experience.” Trends in Cognitive Science 11, no. 5 (2007): 197-203.

Frost, James Edward. "The Serious Game: Towards a Hermeneutic Understanding of the Tarot." The International Journal of the Image 7, no. 2 (2016): 15-32.

Gallagher, Shaun and Dan Zahavi. The Philosophical Mind. New York: Routledge, 2008.

Gavaler, Chris. On the Origin of Superheroes: From the Big Bang to Action Comics No. 1. Iowa City: University of Iowa Press, 2015. 
Gernot, Gerger, Matthew Pelowski, and Helmut Leder. "Empathy, Einfühlung, and Aesthetic Experience: The Effect of Emotion Contagion on Appreciation of Representational and Abstract Art using fEMG and SCR." Cognitive Processing 19, no. 2 (2018): 147-165.

Goldie, Peter. "Anti-Empathy." In Empathy: Philosophical and Psychological Perspectives, eds. Amy Coplan and Peter Goldie, 302-317. New York: Oxford University Press, 2011.

Goodman, Nelson and Catherine Z. Elgin. Reconceptions in Philosophy and Other Arts and Sciences. Indianapolis: Hackett Publishing Company, 1988.

---. The Mess Inside: Narrative, Emotion, and the Mind. Oxford, UK: Oxford University Press, 2014.

Goodwin, Megan. "Conversion to Narrative: Magic as Religious Language in Grant Morrison's Invisibles." In Graven Images, edited by A. David Lewis and Christine Hoff Kraemer, 258-273. New York: Continuum Intl. Publishing Group, 2010 .

Graesser, A.C., K.K. Millis, and R.A. Zwaan. "Discourse Comprehension," Annual Review of Psychology 48 (1997): 163-189.

Gregory, Roberta. Bitchy's College Daze. Seattle: Fantagraphics, 1995.

Groensteen, Thierry. The System of Comics. Jackson, MS: The University Press of Mississippi, 2007.

Hadju, David. The Ten-Cent Plague: The Great Comic Book Scare and How it Changed America. New York: Farrar, Straus, and Giroux, 2008.

Hatfield, Charles. Alternative Comics: An Emerging Literature. Jackson, MS: University Press of Mississippi, 2005.

Hick, Darren Hudson. "The Language of Comics." In The Art of Comics: A Philosophical Approach, edited by Aaron Meskin and Roy T. Cook, 125-144. Malden, MA: Blackwell Publishing, 2012.

Hicks, Jesse. "The Classics: 'The Invisibles."” The Verge. December 22, 2012. https://www.theverge.com/2012/12/22/3793690/the-classicsgrant-morrison-the-invisibles.

Horrocks, Dylan. "Inventing Comics: Scott McCloud's Definition of Comics." Hicksville. June 2001. http://hicksville.co.nz/Inventing\%20Comics.htm. 
James, William. “What is an Emotion?" Mind, 9 (1884): 188-205.

Jones, Gwyneth. "The Icons of Science Fiction." In The Cambridge Companion to Science Fiction, edited by Edward James and Farah Mendlesoh, 163-173. New York: Cambridge University Press, 2003.

Keen, Suzanne. "Fast Tracks to Narrative Empathy: Anthropomorphism and Dehumanization in Graphic Narratives." SubStance 40, no. 1 (2011): 135-155.

Kibuishi, Kazu. Copper. New York: Graphix, 2010.

Kind, Amy. "Putting the Image Back in Imagination." Philosophy and Phenomenological Research 62, no. 1 (2001): 85-109.

Knowles, Christopher. Our Gods Wear Spandex: The Secret History of Comic Book Heroes. San Francisco: Red Wheel/Weiser, 2007.

Kraemer, Christine Hoff. "Promethea: Comics as Spiritual Tool.” 2003. http://www.christinehoffkraemer.com/promethea-exp.html.

Kraemer, Christine Hoff and J. Lawton Winslade. “"The Magic Circus of the Mind': Alan Moore's Promethea and the Transformation of Consciousness through Comics." In Graven Images: Religion in Comic Books and Graphic Novels, edited by A. David Lewis and Christine Hoff Kraemer, 274-291. New York: Continuum Intl. Publishing Group, 2010.

Kripal, Jeffrey J. Mutants and Mystics: Science Fiction, Superhero Comics, and the Paranormal. Chicago: The University of Chicago Press, 2011.

Kukkonen, Karin. Studying Comics and Graphic Novels. Malden, MA: Wiley Blackwell, 2013.

Kulvicki, John. "Knowing with Images: Medium and Message." Philosophy of Science 77, no. 2 (2010): 295-313.

Lange, Carl and William James. The Emotions, edited by Knight Dunlap. Baltimore: Williams \& Wilkins, 1922.

Leger and Reuths. "Doctor Occult: The Ghost Detective." More Fun Comics \#24. New York: National Allied Publications, 1937.

---. "Doctor Occult: The Ghost Detective." In More Fun Comics \#26. New York: National Allied Publications, 1937.

Lewis, A. David. American Comics, Literary Theory, and Religion: The Superhero Afterlife. New York: Palgrave Macmillan, 2014. 
Lewis, C.S. The Weight of Glory. New York: HarperOne, 1980.

“The Living Death!” Tales From the Crypt \#24. New York: E.C. Comics, 1951.

Loeb, Jeph and Tim Sale. Superman for All Seasons. New York: DC Comics, 1999.

Ma, Xiaojuan, Jodi Forlizzi, and Steven Dow. "Guidelines for Depicting Emotions in Storyboard Scenarios.” 8th International Design and Emotion Conference, 2012.

"Magic Works: An Interview with Grand Morrison Part 1." Mondo2000. February 2017. http://www.mondo2000.com/2017/08/14/magic-works-an-interview-with-grantmorrision.

Mar, Raymond A., Keith Oatley and Maja Djikic, and Justin Mullin, "Emotion and Narrative Fiction: Interactive Influences Before, During, and After Reading," Cognition and Emotion 25, no.5 (2011): 818-833.

Matravers, Derek. Fiction and Narrative. New York: Oxford University Press, 2014.

McCauley, Robert. Why Religion is Natural and Science is Not. New York: Oxford University Press, 2011.

McCloud, Scott. Understanding Comics: The Invisible Art. New York: HarperCollins, 1993.

McGinn, Colin. Mindsight: Image, Dream, Meaning. Cambridge: Harvard University Press, 2006.

Meskin, Aaron. "The Ontology of Comics.” In The Art of Comics: A Philosophical Approach, edited by Aaron Meskin and Roy T. Cook, 31-46, Malden, MA: WileyBlackwell, 2012.

Meskin, Aaron, Roy T. Cook, and Frank Bramlett. Introduction to The Routledge Compantion to Comics, 1-6, edited by Frank Bramlett, Roy T. Cook, and Aaron Meskin. New York: Routledge, 2017.

“The Meteor Monster,” Weird Science \#13. New York: Fables Publishing Company, 1950.

Miall, David S., and Don Kuiken. "A Feeling for Fiction: Becoming What We Behold." Poetics 30, no. 4 (2002): 221-241.

Mitchell, Donald W. Buddhism: Introducing the Buddhist Experience. New York: Oxford University Press, 2002. 
Moore, Alan and J.H. Williams III, Promethea: Book One. La Jolla, CA: America's Best Comics, 2000.

---. Promethea: Book Five. La Jolla, CA: America’s Best Comics, 2000.

Moore, Alan, Stephen Bissette, and John Totleben. "The Anatomy Lesson.” The Saga of the Swamp Thing Book One, \#21. New York: Vertigo, 2012.

---. "Rite of Spring." The Saga of the Swamp Thing Book Two, \#34. New York: Vertigo, 2012.

Moore, Alan and Brian Bolland. Batman: The Killing Joke. New York: DC Comics, 1988.

Moore, Alan and Dave Gibbons. Watchmen. New York: DC Comics, 1986-1987.

Moreci, Michael. Roche Limit Volume 1: Anomalous. Image Comics, 2015.

Morris, Tom. "What's Behind the Mask? The Secret of Secret Identities." In Superheroes and Philosophy: Truth, Justice, and the Socratic Way, edited by Tom Morris and Matt Morris, 250-266. Peru, IL: Carus Publishing, 2005.

Morrison, Grant. The Invisibles: The Invisible Kingdom. New York: DC Comics, 2002.

---., Batman R.I.P. New York: DC Comics, 2010.

Nussbaum, Martha. "Emotions as Judgments of Value and Importance.” In Series in Affective Science. Thinking About Feeling: Contemporary Philosophers on Emotions, edited by R. C. Solomon, 183-199. New York, NY, US: Oxford University Press, 2004.

Nyberg, Amy Kiste. Seal of Approval: The History of the Comics Code. Jackson, MS: University Press of Mississippi, 1998.

O’Neil, Denny and Neal Adams. "The Joker's Five-Way Revenge." Stacked Deck: The Greatest Joker Stories Ever Told. New York: DC Comics, 1990.

Pavlova, Marina, Arseny A. Sokolov, and Alexander Sokolov. "Perceived Dynamics of Static Images Enables Emotional Attribution.” Perception 34, no. 9 (2005): 11071116.

Pratt, Henry John. "Making Comics into Film.” In The Art of Comics: A Philosophical Approach, edited by Aaron Meskin and Roy T. Cook, 147-164. Malden, MA: WileyBlackwell, 2012. 
Prinz, Jesse. Gut Reactions: A Perceptual Theory of Emotion. New York: Oxford University Press, 2004.

Proctor, Sam. “Alan Moore: The Art of Magic.” Pagan Dawn. February 12, 2016. http://www.pagandawnmag.org/alan-moore-the-art-of-magic.

Robinson, Jenefer. Deeper Than Reason: Emotion and its Role in Literature, Music, and Art. New York: Oxford University Press, 2005.

Sanders, Joe Sutliff. "Chaperoning Words: Meaning-making in Comics and Picture Books." Children's Literature 41 (2013): 57-90.

Sartre, Jean-Paul. The Imaginary: A Phenomenological Psychology of the Imagination. New York: Routledge, 2004.

Schechtman, Marya. "The Narrative Self." In The Oxford Handbook of the Self, edited by Shaun Gallagher, 394-418. New York: Oxford University Press, 2013.

---. Staying Alive: Personal Identity, Practical Concerns, and the Unity of a Life. New York: Oxford University Press, 2014.

Shermer, Michael. The Believing Brain: From Ghosts and Gods to Politics and Conspiracies---How We Construct Beliefs and Reinforce Them as Truths. New York: St. Martin's Griffin, 2011.

Siegel, Jerry and Joe Schuster. The Superman Chronicles Volume One. New York: DC Comics, 2006.

Simpson, Will. "Feelings in the Gutter: Opportunities for Emotional Engagement in Comics." Image Text: Interdisciplinary Comics Studies 9, no. 4 (2018): http://www.english.ufl.edu/imagetext/archives/v10_1/simpson.

---. "The Mystical Stance: The Experience of Self-Loss and Daniel Dennett's 'Center of Narrative Gravity." Zygon: Journal of Religion and Science 49, no. 2 (2014): 458-475.

Smith, Joel. "Seeing Other People." Philosophy and Phenomenological Research LXXXI, no. 3 (2010): 731-748.

Sousanis, Nick. Unflattening. Cambridge, MA: Harvard University Press, 2015.

Stableford, Brian. "Science Fiction Before the Genre." In The Cambridge Companion to Science Fiction, edited by Edward James and Farah Mendlesohn, 15-31. New York: Cambridge University Press, 2003. 
Star Trek: The Original Series: Season Three. Directed by Herb Wallerstein. 1969; CBS Paramount International Television. 2008, DVD.

Strawson, Galen. “Against Narrativity.” Ratio 17, no. 4 (2004): 428-452.

Steadman, John L. H.P. Lovecraft and the Black Magickal Tradition: The Master of Horror's Influence on Modern Occultism. San Francisco: Weiser Books, 2015.

Suzuki, Shunryu. Zen Mind, Beginner's Mind: Informal Talks on Zen Meditation and Practice, edited by Trudy Dixon. New York: Weatherhill, 2002.

Thompson, Craig. Blankets. Montreal, Canada: Drawn and Quarterly, 2015.

Tilley, Carol L. "Seducing the Innocent: Fredric Wertham and the Falsifications that Helped Condemn Comics." Information \& Culture 47, no. 4 (2012): 383-413.

Trombetta, Jim. The Horror! The Horror!: Comic Books the Government Didn't Want You to Read. New York: Abrams ComicArts, 2010.

Velleman, J. David. Self to Self: Selected Essays. New York: Cambridge University Press, 2006.

Versaci, Rocco. This Book Contains Graphic Language: Comics as Literature. London: Continuum Intl. Publishing Group, 2007.

Versluis, Arthur. "Western Esotericism and Consciousness." Journal of Consciousness Studies 7, no. 6 (2000): 20-33.

---. Magic and Mysticism: An Introduction to Western Esotericism. Lanham, MD: Rowman \& Littlefield Publishers, 2007.

Walton, Kendal. "Fearing Fictionally." In Arguing About Art: Contemporary Philosophical Debates, edited by Alex Neill \& Aaron Ridley, 257-271. New York: Routledge, 2008.

Ware, Chris. Jimmy Corrigan: The Smartest Kid on Earth.New York: Pantheon Graphic Novels, 2003.

Wertheimer, Max. "Laws of Organization in Perceptual Forms." Classics in the History of Psychology. Accessed on March 13, 2016, http://psychclassics.yorku.ca/Wertheimer/Forms/forms.htm.

Wertham, Fredric. Seduction of the Innocent: The Influence of Comic Books on Today's Youth. New York: Rinehart, 1954. 
Wertham, Fredric, and Gerson Legman. "The Psychopathology of Comic Books." American Journal of Psychotherapy 50, no. 4 (1996): 417-434.

Winslade, Jason Lawton. "Enrolling in the 'Hidden School."” In Supernatural Youths: The Rise of the Teen Hero in Literature and Popular Culture, edited by Jes Battis, 197-216. New York: Lexington Books, 2011.

Witty, Paul, Ethel Smith, and Anne Coomer. "Reading the Comics in Grades VII and VIII." Journal of Educational Psychology 33 (1942): 173-182.

Wolk, Douglas. Reading Comics: How Graphic Novels Work and What They Mean. Philadelphia, PA: Da Capo Press, 2007.

Wolverton, Basil. "Nightmare World." Weird Tales of the Future \#3. New York: Key Publications, 1952.

The World Religions: Western Traditions (Fourth Edition), edited by Willard G. Oxtoby, Amir Hussain, and Roy C. Amore. New York: Oxford University Press, 2014.

Wright, Bradford W. Comic Book Nation: The Transformation of Youth Culture in America. Baltimore: The Johns Hopkins University Press, 2001.

Wright, Lucy. "Shamans vs (Super)heroes." In Super/Heroes: From Hercules to Superman, edited by Wendy Haslem, Angela Ndalianis, and Chris Mackie, 127138. Washington, DC: New Academia Publishing, 2007.

Zahavi, Dan. Subjectivity and Selfhood: Investigating the First-Person Perspective. Cambridge, MA: MIT Press, 2005.

Zwaan, Rolf and Gabriel Radavansky. "Situation Models in Language Comprehension and Memory." Psychological Bulletin 123, no. 2 (1998): 162-185. 


\title{
CURRICULUM VITAE
}

\author{
Will D. Simpson
}

www.wdsimpson.com

william.simpson222@gmail.com

\section{EDUCATION}

Ph.D. Humanities - University of Louisville (In Progress :: expected graduation December 2018)

M.A. Religious Studies - Western Kentucky University - 2013

B.A. Philosophy - Western Kentucky University - 2010

B.A. Religious Studies - Western Kentucky University - 2010

\section{A.A. Somerset Community College - 2006}

\section{EXPERIENCE}

Graduate Employee

UNIVERSITY OF LOUISVILLE - 2014-PRESENT

- Responsibilities including: Preparing undergraduate lectures, syllabus, assignment and examination assessment, and student consultation

- Introduction to World Religions (HUM 216)

- Introduction to Western Religions (HUM 219)

\section{Adjunct Faculty}

INDIANA UNIVERSITY SOUTHEAST - SPRING 2014

- Responsibilities including: Complete course development and oversight

○ Introduction to Ethics (PHIL-P 140)

\section{Instructor of Record}

WESTERN KENTUCKY UNIVERSITY - SPRING 2013

- Responsibilities including: Complete course development and oversight

- Introduction to Religion (RELS 102)

\section{Graduate Teaching Assistant}

WESTERN KENTUCKY UNIVERSITY - 2011-2013

Responsibilities including: Exam and essay grading, leading student discussion groups, assisting professors in any necessary capacity 
Assistant to the Editor

INTERNATIONAL JOURNAL OF RELIGION AND SPORT- 2012-2013

- $\quad$ Responsibilities including: composing book reviews, proof-reading submitted articles, and providing article submission assessments

\section{PUBLICATIONS AND CONFERENCES}

"Feelings in the Gutter: Opportunities for Emotional Engagement in Comics" IMAGETEXT VOL 10, NO. 1 - SEMPTEMBER 2018

"Review of On the Origin of Superheroes: From the Big Bang to Action Comics No. 1 by Chris Gavaler." STUDIES IN COMICS VOL 8, NO. 1 - JULY 1, 2017

"Review of American Comics, Literary Theory, and Religion: The Superhero Afterlife by A. David Lewis." JOURNAL OF RELIGION AND POPULAR CULTURE - IN PRESS

"Souls, the Final Frontier: Human Intuitions of Mind in Star Trek" JOURNAL OF RELIGION AND POPULAR CULTURE VOL 18, NO. 2-3 - FALL 2016

"The Mystical Stance: The Experience of Self-Loss and Daniel Dennett's 'Center of Narrative Gravity'” ZYGON: JOURNAL OF RELIGION AND SCIENCE - MAY 21, 2014

"True Religion, True Spirituality, True Detective" RELIGIONNERD.COM ARTICLE - MARCH 26, 2014

“Revisiting Sacred Metaphors: A Religious Studies Pedagogical Response to the Rise of the Nones" JOURNAL OF RELIGION AND SOCIETY - JANUARY 2014

"Similar Differences: A Compatibilist View of the Common Core Debate in the Study of Mystical Experience" AXIS MUNDI (UNIVERSITY OF ALBERTA) - JULY 18, 2013

The Louisville Conference: Panel Chair - Presenter

"Picturing Yourself: Graphic Narratives and Selfhood" FEBRUARY 22, 2018

The Louisville Conference: Presenter

"Feelings in the Gutter: Opportunities for Emotional Engagement in Comics" FEBRUARY 23, 2017

Midwest Popular and American Culture Association Annual Conference: Panel Chair - Presenter “American Kryptonite: Superman and the Disenchantment from Civil Religion in the U.S." OCTOBER 6, 2016

Kentucky Philosophical Association Annual Conference: Responder APRIL 16, 2016

Southeastern Commission for the Study of Religion: Presenter "Rethinking Sacred Metaphors in Response to the Rise of the Nones" MARCH 7-9, 2014 
Columbus State University Graduate Research Conference: Presenter

"Similar Differences: A Compatibilist View of the Common Core Debate in the Study of Mystical

Experiences"

OCTOBER 25, 2012

WKU Undergraduate Research Conference: Presenter

"Particularism and Defining Morality" FEBRUARY 27, 2010

\section{CERTIFICATIONS AND ACKNOWLEDGMENTS}

Doctoral Dissertation Completion Award - UNIVERSITY OF LOUISVILLE - FALL 2018

Active Learning Workshop Series - UNIVERSITY OF LOUISVILLE - 2017-2018

The Grant Writing Academy - UNIVERSITY OF LOUISVILLE - 2017

Potter College Graduate Award - EXCELLENCE IN SCHOLARSHIP FOR RELIGIOUS STUDIES, WKU - APRIL 2013

The Basic Skills for College Teaching Program - WKU - 2012

\section{MEMBERSHIPS AND AFFILIATIONS}

Association of Humanities Academics Secretary (U of L): 2016-2018

Midwest Popular Culture Association: 2016-2017

Kentucky Philosophical Association: 2016-2017

American Academy of Religion: 2012-2014

Theta Alpha Kappa: 2010-Present

Kentuckians for the Commonwealth: 2010-2012

Secular Student Alliance - WKU Chapter Secretary: 2012-2013

WKU Interfaith Student Project Collaborator: 2012-2013

\section{REFERENCES}

John Gibson, PhD - Professor of Philosophy - University of Louisville JOHN.GIBSON@LOUISVILLE.EDU (502) 852-0452

Andreas Elpidorou, PhD - Assistant Professor of Philosophy - University of Louisville ANDREAS.ELPIDOROU@LOUISVILLE.EDU

Eric Bain-Selbo, PhD - Dean of School of Humanities and Social Sciences - Indiana University Kokomo EBAINSEL@IU.EDU (765) 455-9280 